\title{
Aspects of QCD uncertainties and fast QCD predictions for high-energy collider experiments
}

\author{
Dissertation \\ zur Erlangung des mathematisch-naturwissenschaftlichen Doktorgrades \\ „Doctor rerum naturalium“ \\ der Georg-August-Universität Göttingen \\ im Promotionsprogramm ProPhys \\ der Georg-August University School of Science (GAUSS) \\ vorgelegt von \\ Enrico Bothmann \\ aus Berlin
}

Göttingen, 2016 
Betreuungsausschuss

Jun.-Prof. Dr. Steffen Schumann

II. Physikalisches Institut, Georg-August-Universität Göttingen

Prof. Dr. Arnulf Quadt

II. Physikalisches Institut, Georg-August-Universität Göttingen

Mitglieder der Prüfungskommission:

Referent: $\quad$ Jun.-Prof. Dr. Steffen Schumann

II. Physikalisches Institut, Georg-August-Universität Göttingen

Koreferent: $\quad$ Prof. Dr. Arnulf Quadt

II. Physikalisches Institut, Georg-August-Universität Göttingen

Drittgutachter: Prof. Dr. Luigi Del Debbio

School of Physics and Astronomy, University of Edinburgh

Weitere Mitglieder der Prüfungskommission:

Prof. Dr. Laura Covi

Institut für Theoretische Physik, Georg-August-Universität Göttingen

Prof. Dr. Jens Niemeyer

Institut für Astrophysik, Georg-August-Universität Göttingen

Prof. Dr. Ansgar Reiners

Institut für Astrophysik, Georg-August-Universität Göttingen

Dr. Frank Siegert

Institut für Kern- und Teilchenphysik, Technische Universität Dresden

Tag der mündlichen Prüfung: 


\title{
Aspects of QCD uncertainties and fast QCD predictions for high-energy collider experiments
}

\author{
Enrico Bothmann
}

\begin{abstract}
We address the issue of how to provide accurate perturbative QCD predictions along with their theory uncertainties in a systematic and fast way using Monte-Carlo event generators, considering that state-of-the-art calculations become more and more complex and require in some cases prohibitively extensive central processing unit (CPU) resources. An internal reweighting method is presented for the SHERPA event generator. It gives variations to a nominal prediction with comparably little additional computational cost. The method supports next-to-leading-order multi-jet calculations in QCD combined with allorder corrections generated with a parton shower. Moreover, we present new developments for another reweighting approach, namely the use of QCD interpolation grids. These provide even faster variations for fixed-order calculations, and can be created in an automated way using event generators through interfaces such as MCGRID. Our improvements to this interface allow for creating more versatile interpolation grids, supporting a larger class of calculations, grid implementations and scale variations. Furthermore, we discuss ideas for a future inclusion of resummation effects in such grids. Besides the reweighting, we also study the use of extrapolation methods to predict high-multiplicity jet rates, which are expected to be ubiquitous at future collider energies. The extrapolation is based on scaling patterns. This study is embedded in a more general discussion of jet activity at a future $100 \mathrm{TeV}$ proton-proton collider.
\end{abstract}

\author{
II.Physik-UniGö-Diss-2016/o4 \\ II. Physikalisches Institut \\ Georg-August-Universität Göttingen
}




\title{
Aspekte von QCD-Unsicherheiten und von schnellen QCD-Vorhersagen für Experimente an Hochenergiebeschleunigern
}

\author{
Enrico Bothmann
}

\section{Zusammenfassung}

In dieser Arbeit adressieren wir die Schwierigkeit, Präzisionsvorhersagen mit dem kompletten Satz theoretischer Unsicherheiten in der perturbativen Quantenchromodynamik im Rahmen von Monte-Carlo-Simulationen zu treffen, angesichts der zunehmenden Komplexität der dazu nötigen Berechnungen. Die Anforderungen an die Rechenleistung können so groß sein, dass nicht in jeder Anwendung die bestmögliche Präzision erzielt wird. Wir präsentieren eine Reweighting-Methode für den Monte-Carlo-Ereignisgenerator SHERPA. Diese erstellt Variationen der nominellen Vorhersage mit vergleichsweise geringem zusätzlichen Zeitaufwand. Die Methode ist kompatibel mit aktuellen Multijet-Berechnungen nächsthöherer Ordnung, die mit Korrekturen von allen Ordnungen durch einen Partonschauer versehen sind. Zusätzlich diskutieren wir neue Entwicklungen für einen weiteren Reweighting-Ansatz, der auf QCD-Interpolationsgittern beruht. Diese ermöglichen noch schnellere Variationen für Berechnungen fester Ordnung. Solche Gitter können für MonteCarlo-Simulationen automatisiert erstellt werden mithilfe von Interfaces wie MCGRID. Unsere Verbesserungen für MCGRID ermöglichen die Erstellung vielseitigerer Gitter, die eine größere Klasse von Berechnungen, Gitter-Implementierungen und Skalenvariationen unterstützen. Darüber hinaus diskutieren wir, auf welche Weise solche Gitter für die Unterstützung von Resummationseffekten erweitert werden müssten. Neben dem Reweighting studieren wir noch die Verwendung von Extrapolationsmethoden für die Vorhersage von Jet-Raten hoher Multiplizitäten, welche an zukünftigen Hochenergiebeschleunigern allgegenwärtig sein werden. Diese Methoden basieren auf dem Skalierungsverhalten der Jet-Raten. Eingebettet ist diese Studie in eine allgemeinere Diskussion der zu erwartenden Jet-Aktivität an einem Proton-Proton-Beschleuniger mit einer Schwerpunktsenergie von $100 \mathrm{TeV}$. 


\section{Contents}

1 Introduction 1

2 Elements of Monte-Carlo event generation 10

2.1 The Sherpa framework 10

2.1.1 Physics modules 10

2.1.2 Standard Model choices 14

2.2 The state-of-the-art in perturbative QCD calculations $\quad 14$

2.2.1 Pure fixed-order calculations 14

2.2.2 Exclusively adding log-enhanced emissions - the parton shower 16

2.2.3 Matching NLO calculations with a parton shower 17

2.2.4 Merging multiple jet multiplicities 18

2.2.5 Towards NNLO 20

2.3 QCD uncertainties in the context of event generators 21

2.4 Conclusive closure tests 23

2.4.1 Controlling Monte-Carlo statistics 23

2.4.2 Basic validations with auto-closure tests 24

2.4.3 Cross-closure tests and uncertainty band comparisons 24

3 Reweighting (N)LO matrix elements and parton showers 26

$3.1 \quad$ Fixed-order calculations 27

3.1.1 The leading-order case 27

3.1.2 The next-to-leading-order case 28

3.2 Closure tests for fixed-order calculations 30

3.3 Parton showers 31

3.3.1 Sudakov form factors 31

3.3.2 The (reweighted) Sudakov Veto Algorithm 33

3.3.3 Cut-off issues and numerical instabilities 36

3.4 LO matrix elements and parton showers 40

3.5 NLO matrix elements and parton showers 44

3.6 Closure tests for (N)LO matrix elements and parton showers 46

3.6.1 The final-state only case: Thrust and jet resolutions in $\mathrm{e}^{+} \mathrm{e}^{-} \rightarrow \mathrm{q} \overline{\mathrm{q}}$ events 47

3.6.2 The initial-state dominated case: $\mathrm{W}$-boson transverse momentum and jet resolutions in $\mathrm{pp} \rightarrow \mathrm{W}\left[\mathrm{e}^{-} v\right]$ events 49 
3.7 Multi-jet merging 54

3.8 The leading-order case: MEPS@LO 57

3.9 The next-to-leading-order case: MEPS@NLO 59

3.10 Closure tests for multi-jet merged calculations 62

3.11 Adding reweighted emissions vs. merging more multiplicities 67

3.12 Using over-estimate factors for a smoother parton-shower reweighting 68

3.13 Preserving the formal accuracy in variations 72

3.14 CPU time measurements 73

3.15 Discussion 75

4 QCD interpolation grids 79

4.1 Encoding cross sections on interpolation grids 80

4.1.1 Multiplicative $\alpha_{S}$ and PDF dependences 80

4.1.2 Scale logarithms: RGE approach vs. dedicated grids 82

4.1.3 LO \& NLO plus parton-shower calculations 83

4.1.4 Optimised subprocess bases 83

4.2 Automation for Catani-Seymour subtracted NLO calculations via MCgrid 84

4.3 Closure tests for fixed-order calculations 85

4.4 Comparison of scale-logarithm methods 86

4.5 Comparison between MCgrid-filled APPLgrids and fastNLO grids 87

4.6 Comparison to the internal reweighting including the parton shower 90

4.7 Future steps towards beyond fixed-order interpolation grids 90 4.7.1 Multi-jet merging via stacked interpolation grids 91

4.7.2 Approximate approaches to include parton-shower emissions 92

4.8 Discussion 93

5 QCD uncertainties and jet rate extrapolations at a future $100 \mathrm{TeV}$ protonproton collider 95

5.1 QCD cross sections at high energies 96

5.2 The scaling behaviour of multi-jet rates 100

5.3 Discussion 109

6 Conclusions 111

A Configuring and accessing event-weight variations 114

Bibliography 117

Acknowledgements $\quad \mathbf{1 2 8}$ 


\section{Introduction}

The first operational run of the Large Hadron Collider (LHC) from 2009 to 2013, Run 1, provided an integrated luminosity of about $30 \mathrm{fb}^{-1}$ of proton-proton collision data at $7-8 \mathrm{TeV}$. Many important physics results were, and are still, being obtained from it. The most notable example is the discovery of a Higgs boson by the two collaborations A Toroidal LHC ApparatuS (ATLAS) and Compact Muon Solenoid (CMS) $[1,2]$ in 2012. This discovery and a large number of other experimental analyses, focusing on a variety of final states and observables, (re-)established and underpinned the validity of the Standard Model of particle physics (SM) [3] to an unprecedented level of precision.

Searches for resonances in otherwise smooth invariant-mass distributions, such as the resonance at the Higgs mass of $125 \mathrm{GeV}$ in the di-photon channel, are among the first physics analyses of collision data at new energy frontiers, because they can be successful even with limited statistics. Later, with sufficient collision data being available, and statistics not being the limiting factor any more, the focus shifts towards precision physics: searches for less- or non-resonant deviations from theory predictions, and precision measurements of model parameters. To make most of the available data, the systematic uncertainties should at least be comparable to the statistical uncertainty. Otherwise, new physics might hide in our imperfect knowledge of both the theory and the experimental set-up. While the data taken during Run 1 are still being analysed, Run 2 has started very successfully and is expected to provide about $100 \mathrm{fb}^{-1}$ of collision data at $13-14 \mathrm{TeV}$. Moreover, a proposal for a HighLuminosity LHC has a design goal of $3 \mathrm{ab}^{-1}$ [4]. Long-term, a Future Circular Collider (FCC) is discussed as one of several future collider proposals with proton-proton collisions at a centre-of-mass energy of $100 \mathrm{TeV}$ and an integrated luminosity of $1-10 \mathrm{ab}^{-1}$ [5-8]. Current and future experiments will be even greater opportunities to analyse very large data sets; the precision era of hadronic colliders is only beginning to unfold.

A prime example, and perhaps the most precise result of today, is one of the standard candles of collider phenomenology, the Z-boson transverse momentum $\left(p_{T}^{\mathrm{Z}}\right)$ measurement, see Fig. 1.1. When the distribution is normalised to the fiducial cross-section of Drell-Yan production $^{1}$, the experimental uncertainties are below $\pm 1 \%$ for $p_{T}^{\mathrm{Z}}<200 \mathrm{GeV}$. With its ten-fold increase in data, Run 2 will bring more results down to this level of precision. And for the High-Luminosity LHC, a simple extrapolation with respect to the increased luminosity indicates that the couplings of the Higgs boson to fermions and other bosons can be measured with a 1-4\% precision [9]. A recent study concludes that the observation of di-Higgs production at the High-Luminosity LHC in the bb̄bb decay channel requires

${ }^{1}$ By a normalisation with respect to the total cross section, the uncertainties for the luminosity and some lepton efficiency systematics cancel. 


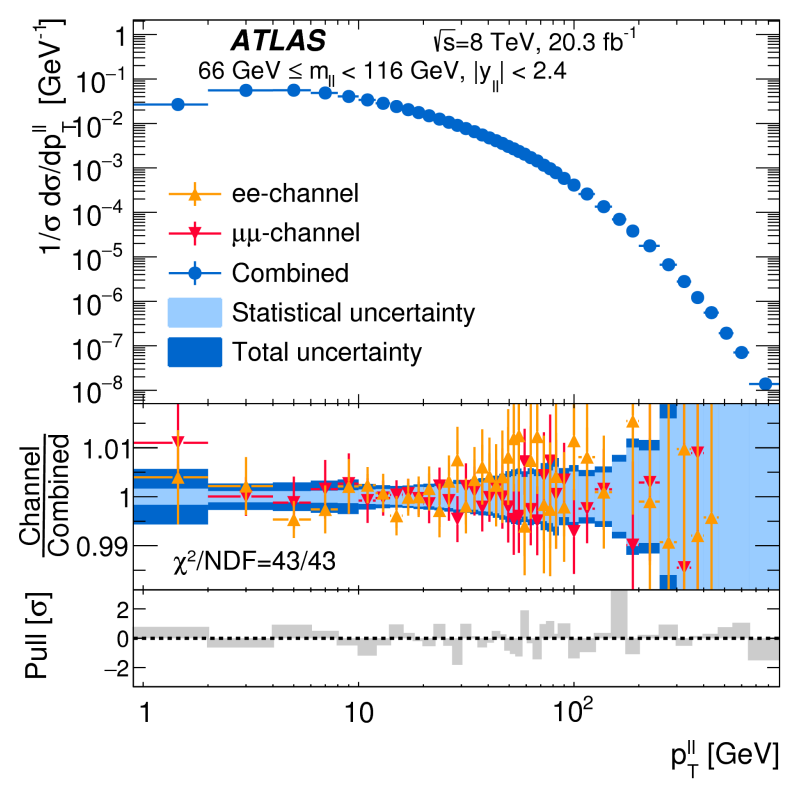

Figure 1.1.: A measurement of the Z-boson transverse momentum in its $\mathrm{e}^{+} \mathrm{e}^{-}$and $\mu^{-} \mu^{+}$decay channels. Figure taken from [11].

controlling the backgrounds at a $1 \%$ level [10].

Precise results such as the one for $p_{T}^{Z}$, and the projections of experimental precisions in the mid-term and long-term future, are a challenge for theory predictions in general, and in particular for the theory of the strong interactions, Quantum Chromo-Dynamics (QCD). Most predictions for the LHC are still limited by the accuracy of our QCD calculations, although tremendous progress has been achieved. The size of the strong coupling with $\alpha_{S} \approx 0.1$ at the Z-boson mass means its perturbative convergence is slower than e.g. for Quantum Electro-Dynamics (QED). Another difference to QED is the non-Abelian group structure of QCD, that allows for gauge-boson self-interactions. The consequence of a large coupling and these self-interactions is that additional QCD radiation comes with large rates. Partonic jets are formed from cascades of partons that originate from a single mother parton. The non-Abelian group structure also leads to the phenomenon of confinement, which prohibits the exposure of colour charges at large length scales. Hence, the partons eventually cluster into colour-neutral initial- and final-state hadrons. This process is called fragmentation in the final state. The partonic jets are taken into hadronic jets that enter the detector as sprays of hadrons that share a similar direction. In the initial state, the colour-neutral objects are the colliding protons from the incoming beams, and the proton structure in terms of partons is parametrised by parton density functions (PDFs). Due to these complications, the analysis of the hard interaction using hadronic observables is more complex than is the case for leptons or photons. It requires a good understanding, or at least modelling, of the different steps in the evolution from the high energy scale of the 
hard interaction down to the much lower energies at which the fragmentation takes place. Perturbation theory is at the centre of QCD predictions at high-energy colliders, with an expansion of the matrix element in $\alpha_{S}$. Such perturbative QCD (pQCD) calculations can either be combined with analytic all-order resummations of logarithm-enhanced terms in the perturbative expansion, or embedded within Monte-Carlo event generators (MCEGs), which simulate entire collision events, including a numerical resummation of some enhanced terms using parton-shower algorithms and a modelling of non-perturbative effects such as fragmentation.

Pure fixed-order calculations are fully automated to next-to-leading-order (NLO) accuracy, and more and more next-to-next-to-leading-order (NNLO) results becoming available. Indeed, for most standard processes, the total cross section at NNLO is now known, and many cross sections for differential observables have followed, with a plethora of new results in the last 2 years alone [12-19]. Although there are still major problems to be solved for a full automation of NNLO calculation, as discussed in Section 2.2.5, it has become conceivable that this task might be completed in the near future. However, pure fixed-order calculations at any order have a limited applicability at colliders, and in particular at hadron colliders, because of the implicit integration over the $\mathrm{PQCD}$ evolution at lower energies. As a consequence, also a combination with current non-perturbative models is not possible. These require the full specification of the initial- and final-state partons at the length scales at which their fragmentation takes place. Fixed-order calculations (in combination with analytic resummations) can be very successfully applied to observables that are only sensitive to final-state leptons or, more generally, are inclusive with respect to the evolution of finalstate partons into hadrons (with the most inclusive example being the total cross-section). However, less inclusive ones will eventually suffer from missing non-perturbative corrections and the inherently non-exclusive description of additional jet emissions. Moreover, at hadron colliders even observables defined for QCD-free final states are affected by the unavoidable QCD interactions in the initial state, e.g. through the recoil that is built up via QCD emissions off the incoming partons.

MCEGs are simulation tools with a more comprehensive approach. They start with a fully differential fixed-order calculation for the hard process and use parton-shower algorithms to evolve the initial- and final-state partons down to a cut-off scale with fully specified kinematics at each step. At the cut-off scale, the non-perturbative fragmentation into hadrons is simulated using universal phenomenological models. In addition, they model the evolution of the remnants of the incoming hadrons, including additional parton interactions among them. All of these aspects are combined to give a complete description of individual pseudo-events, with a full specification of the final-state as it would enter the detector in the real world. Hence, they are most generally applicable for collider physics. With their help, experimental consequences of theoretical models can be tested, by a comparison of real data with pseudo-data, for which the MCEG simulation is potentially complemented by a detector simulation. MCEGs are also used to develop search strategies, to find interpretations for data patterns, and to study the detector performance. As such, they are an essential tool during the whole lifetime of a collider experiment, from early conceptual studies to the 
evaluation of the data.

While the first iterations of MCEGs were only applicable to single leading-order calculations for the hard interaction, and had rather simple parton showers based on a leadinglogarithmic description of collinear emissions, they have since then matured into precision tools. Modern parton showers incorporate soft-gluon emissions by correctly accounting for colour-coherence effects [20-23]. Their combination with NLO matrix elements has been fully automated ("matching") [24, 25], and the hardest jet emissions can be systematically replaced with exact matrix-element calculations ("multi-jet merging") [26-33] combining the power of multiple matrix elements and the parton shower in one inclusive sample. Both matching and merging to LO and NLO have been finalised only in the last 15 years (with NLO merging in 2012 [33]), and allow the use of MCEGs for fully automated and precise predictions. The next natural step clearly is to promote the matching and merging schemes to NNLO, without which a general $\mathcal{O}(1 \%)$ accuracy is inconceivable. For first processes, a matching of NNLO matrix elements to parton showers have already been achieved [34-39], but the full automation, in particular to colourful final-states, might take a few more years. ${ }^{2}$

With the description of the available tools and their latest developments, we can return to the question of theoretical accuracy, and how it compares to the requirements of the precision era of hadron colliders. Naively, the perturbative uncertainty ${ }^{3}$ at the order $p$ is given as $\alpha_{S}^{p} \approx 0.1^{p}$ (although some processes such as gluon-fusion Higgs production defy this rule). Hence, with going from NLO to NNLO we move from perturbative uncertainties around $10 \%$ down to about $1 \%$. For the Z-boson transverse momentum with its purely leptonic signature, a comparison to a pure matrix-element calculation seems viable. For this observable $p=1$, as the recoil off a parton emission is needed for $p_{T}^{\mathrm{Z}} \neq 0$. In fact, a remarkable perturbative accuracy of $\pm 1.5 \%$ has been achieved for the $p_{T}^{Z}$ distribution [18].

Besides perturbative uncertainties, fixed-order calculations also suffer from parametric uncertainties, because of the free parameters in QCD, namely the quark masses, the strong coupling $\alpha_{S}$, and at hadron colliders the parametrisation of the parton content of the hadrons, the PDFs. ${ }^{4}$ They are determined by fits of QCD calculations to experimental data. Hence, their accuracy is not independent of other theoretical (and experimental) uncertainties. Part of a successful precision era program will be new measurements, especially of $\alpha_{S}$ and the PDFs, as they enter nearly all hadronic collider predictions. Currently, their uncertainties in central phase-space regions are of the order of $1 \%\left(\alpha_{S}\right)$ and $2-3 \%$ (PDFs), although tensions exist between fits, and with respect to data comparisons. This again highlights the need for further investigations and new fits. In fact, one example is the Z-boson

\footnotetext{
${ }^{2}$ The incorporation of NNLO calculations and future colliders with higher energies will make the incorporation of electro-weak NLO corrections necessary. Automated combinations of NLO QCD and NLO electro-weak corrections for matrix-element plus parton-shower calculations have recently become available [40].

${ }^{3}$ The perturbative uncertainty is due to the corrections of missing higher-order terms. Hence, it can only be estimated, as we will discuss in Chapter 2.

${ }^{4}$ Strictly speaking, PDFs are not free parameters, but (partly) non-perturbative quantities that are in principle calculable from first principles, as is being attempted using lattice QCD methods [41]. However, until this is achieved, PDFs are subject to fits the same way as $\alpha_{S}$ is, and hence its uncertainty is categorised as parametric here.
} 
transverse momentum distribution, where the NNLO prediction is consistently about $4 \%$ smaller than the experimental results. The tension is also present in the total cross section, and it has been proposed to constrain the high- $x$ gluon PDF [19] with the total cross section, or the $p_{T}^{\mathrm{Z}}$ distribution.

In MCEGs, both perturbative and parametric uncertainties are still present in the hardinteraction matrix-element calculation, but enter the calculation also through the $\mathrm{PQCD}$ parton-shower algorithm. Moreover, the matching and merging schemes of the matrix element(s) with the parton shower further complicates the dependence structure. These schemes and the parton shower itself also offer some choices within their perturbative accuracy, which introduces further algorithmic uncertainties. Although they should formally not exceed the perturbative uncertainty, numerics or an enhancement of higher-order differences in certain phase-space regions can lead to sizeable deviations. In addition, the fit parameters and assumptions of the non-perturbative models are another source of uncertainty in MCEGs. Hence, for uncertainty studies, MCEGs are a much more complex environment than a pure fixed-order calculation, even if we restrict ourselves to perturbative and parametric uncertainties, of which the former dominate at (N)LO.

Multi-jet merging allows for a systematic reduction of perturbative uncertainties by including matrix elements for additional jet multiplicities, or by replacing LO matrix elements with NLO ones. Of course, one can not formally exceed NLO accuracy this way, but with each additional matrix-element the region in which jets are described to (N)LO expands. Some recent examples are:

1. the leading-jet transverse momentum in top-quark pair production, where with LO matrix elements for the 0-, 1- and 2-jet multiplicities the perturbative uncertainties are around $100 \%$, whereas when promoting the three matrix elements to NLO, the uncertainty goes down to about $20 \%$ [42];

2. observables related to the hardest jet in the production of 4 leptons, where with a combination of NLO matrix elements for the o- and 1-jet multiplicities the perturbative uncertainty was reduced to about $5 \%$ compared to an uncertainty of about $10-15 \%$ when a single NLO matrix element was used [43];

3. and the number of jets $N_{\text {Jets }}$ and the azimuthal angle $\Delta \Phi$ between the two leading jets in W-boson production. In Fig. 1.2, we present results from [33], where the distributions are compared for Matrix Elements merged with Parton Shower emissions at Next-toLeading Order (MEPS@NLO) (W+ o, 1, 2 jets at NLO, and W + 3, 4 jets at LO), Matrix Elements merged with Parton Shower emissions at (Next-to-)Leading Order (MENLOPS) (W + o jets at NLO, $\mathrm{W}+1,2,3,4$ jets at LO) and MC@NLO (W + o jets at NLO, and W + 1 jets at LO). For MEPS@NLO and MENLOPS scale variations are employed to estimate the perturbative uncertainty. All predictions are compared to ATLAS data [44]. The MC@NLO prediction fails to describe the two observables over the complete phase space. The MENLOPS is systematically smaller than the data, but with its large uncertainties (about 50 \%) it is still compatible. The MEPS@NLO has much lower uncertainties 

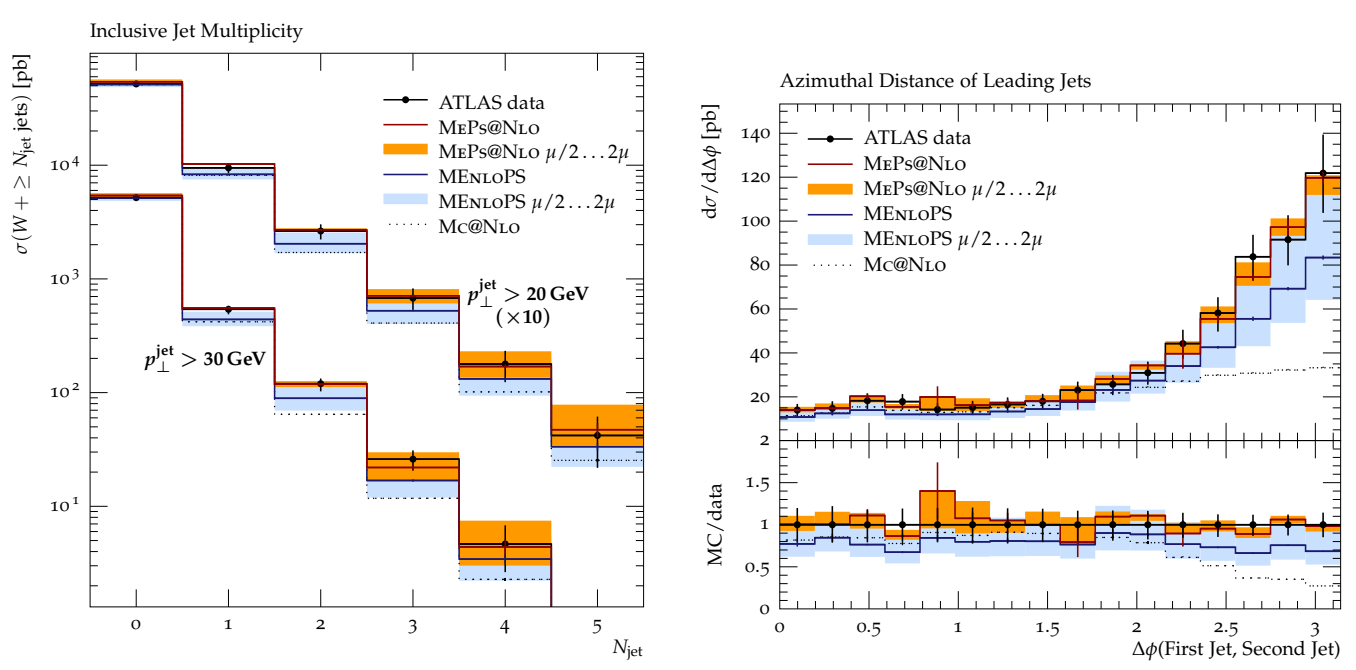

Figure 1.2.: A comparison of predictions for the inclusive jet multiplicity and the azimuthal distance of the two leading jets in W-boson + jets production in pp collisions at a centre-ofmass energy of $7 \mathrm{TeV}$. A parton shower is combined with a varying number of jets described by matrix elements at LO and NLO, as described in Example 3 on Page 5. Figures taken from [33].

(about $10 \%$ ), wherever the phase space is well-described by up to 2 jets. When more jets are required to describe the observable $\left(N_{\text {Jets }} \geq 3\right.$ or $\left.\Delta \Phi \leq 2 \pi / 3\right)$, the differences in the accuracy begin to vanish, as expected.

From these examples, we can see that the inclusion of NLO matrix elements leads to a dramatic reduction of perturbative uncertainties and a better description of data in MCEG simulations. With NLO multi-jet merging in particular, a current standard of about $10-20 \%$ uncertainty has been established.

The ongoing developments to provide accurate predictions do not come for free. With more complicated calculations, the CPU time needed for their evaluation grows. And with better perturbative accuracy, previously neglected uncertainty sources need to be taken into account. Often, the only way to do this is to repeat the calculation with variations in the choices that are connected to the uncertainties, with the consequence of even higher resource needs, in CPU time and possibly in (pseudo-)data storage. Experiments such as ATLAS and CMS perform massive central Monte-Carlo event generations, and need to store event data that are of a considerable size even without considering the proliferation through variations. Detector simulations for these event data records are so CPU-intense that a repetition for a large number of variations is prohibitive. Another prime example where explicit variations do not meet the time requirements are PDFs fits. These need tens of thousands of PDF variations for a complete fit, which are not known a priori, but only after each fitting iteration. 
These problems exist because theory needs to accommodate better statistical accuracies in current and future colliders. The other main collider development, which is to increase higher centre-of-mass energies, is an additional challenge for QCD predictions. Although higher jet multiplicities come with a huge increase in calculational complexity, they will occur at large rates at colliders such as the FCC. Searches for new physics will eventually target large jet multiplicities, because they are a natural consequence of decay chains from speculative particles with very high masses.

A major part of the solution to both problems is to provide, extend and study methods that can provide $\mathrm{PQCD}$ predictions without doing the full $\mathrm{PQCD}$ calculation, and thus to overcome CPU time and data storage constraints. This is the aim of this thesis.

Our first focus will therefore be PQCD reweighting methods. These identify the dependences of the parameters or scales that are to be varied, and reuse the independent pieces ("weights") for the variations. Hence the weights are calculated only once. As they are also usually the most CPU-intense pieces, most of the CPU time for calculating variations is saved. Another advantage of these methods stems from the fact that the phase-space point of each event is kept the same, such that the variations are to a large degree statistically equivalent to the nominal calculation, which gives smooth uncertainty bands. This also leads to reduced storage requirements if individual events and their variations need to be stored. In fact, only a single number (the varied event weight) needs to be stored per variation, whereas the final-state specification is shared between the nominal result and its variations. This also removes the need to do more than one detector simulation. As the weight is only associated with the relative probability of the event, its variations do not affect the simulation, and are needed only when fully simulated events are eventually compared, as e.g. in a histogram for some observable.

The main part of this thesis is devoted to the presentation of a first comprehensive PQCD reweighting account, which treats dependences that enter through the hard interaction (for LO and NLO matrix elements) and the parton shower individually, and through their combination by the matching and merging schemes used to combine both. This exceeds previously available methods, which were restricted to (N)LO matrix elements only [45]. We implemented the reweighting as an on-the-fly event-by-event reweighting in the MCEG SHERPA $[46,47]$. The reweighting of parton showers has sparked interest throughout the MCEG community, such that alternate parton-shower reweighting implementations have been reported by the HeRwig $[48,49]$ and Pythia [50] collaborations during the last year of this thesis, with the same underlying algorithm. These do not address the issues of matching and merging and are therefore restricted to the combination with LO matrix elements.

A variant to the event-by-event reweighting is the use of $\mathrm{PQCD}$ interpolation grids, as implemented in the APPLGRID [51] and FAsTNLO [52, 53] packages. Here, the independent weights are written to discrete grids, after the event is projected onto a predefined observable. The individual event information is lost by this projection, such that the resulting grid is specific to the observable. The advantage in comparison to an event-by-event 
reweighting is that much less time is needed for each variation. This makes interpolation grids a perfect tool for PDF fits. Recently, tools for an automated production of interpolation grids have been reported, MCGRID [54-56] and AMCFAST [57]. We present here new developments within the MCGRID tool, most importantly extending its scope to support the fixed-order expansion of NLO calculations matched with a parton shower, as a first step towards interpolation grids with parton-shower support.

By overcoming limitations of both the event-by-event reweighting and interpolation grids, we thus present major improvements to the $\mathrm{PQCD}$ reweighting methodology.

Our second focus takes us away from reweighting methods. Instead, we present an application of another method to overcome otherwise infeasibly lengthy calculations, namely an extrapolation of jet rates to higher multiplicities. For this extrapolation, two scaling patterns in these jet multiplicity distributions are used that appear in different kinematic regimes. These patterns, Staircase and Poisson scaling, have both been observed in LHC data [58-61] and in Monte-Carlo studies [62-64]. They have been explained as limiting cases of jet-emission probabilities [65-67]. The Poisson scaling features a single, hard quark line emitting many gluons, which leads to a Poisson distribution in the jet multiplicities, akin to hard electron lines emitting photons [68]. The Staircase scaling is found when all partons share a similar low virtuality. In this case, gluon emissions off other gluons dominate. As such, Staircase scaling is a distinctive feature of the non-abelian group structure of QCD that allows for gauge-boson self-interactions. We find using Monte-Carlo studies for a FCC set-up that the rates for the first few jet multiplicities can be used to make fits that predict rates for up to 15 or more jets.

The structure of this thesis is as follows. First, in Chapter 2, we introduce a collection of material that is relevant to all later chapters. After explaining the structure and paradigms of a MCEG for the example of SHERPA, we continue with a more systematic discussion of PQCD calculations, and of QCD uncertainties in the context of MCEGs. We end the chapter by reviewing our closure test method to validate the reweightings and extrapolations presented later.

We move on in Chapter 3 to present the first reweighting method with full NLO matrixelement plus parton-shower support. This is the main part of this thesis. After the reweighting of each calculational mode is discussed, we present the validation of its implementation. Additional studies discuss the replacement of parton-shower emissions with matrix-element calculations in a multijet-merged calculation from the perspective of the reweighting, as well as possible improvements of the numerical stability of the parton-shower reweighting. After a short note on whether certain parameter variations retain the formal accuracy of the PQCD calculation, we end the chapter with a study of the CPU time savings.

In Chapter 4, we discuss developments with respect to interpolation grids. After a short review of interpolation grids and their automated generation using the MCGRID tool, we provide validations of the different improvements that we developed in MCGRID. After studying the effect of not reweighting parton-shower emissions through a comparison to dedicated calculations and to the event-by-event reweighting method, we discuss possible 
extensions of interpolation grids with respect to multi-jet merging and parton showers.

Finally, we study jet rates and their extrapolation to higher multiplicities in Chapter 5. After establishing that the FCC is an environment with huge jet rates that extend to large transverse momenta, we study the application of jet rate extrapolations through fits to scaling patterns. 


\section{Elements of Monte-Carlo event generation}

The need for new or improved physics models to be readily available for comparison against data, led to the development of a modular structure of multi-purpose Monte-Carlo event generators (MCEGs). Each module corresponds to a separate physics regime. These regimes factorise from each other through their separation in length/energy scales or simply through a separation in position space. By representing this in the software architecture, new developments in the associated physics models can be easily implemented by a local modification or the replacement of a module.

A cornerstone of event generators is the generation of single collision events with finalstate particles that can actually be measured with the current detector technology. Therefore one speaks of Monte-Carlo (pseudo-)data. This makes it possible to test both established and hypothetical interaction models against real data, with the same tool set. This comparison requires to process the Monte-Carlo event with a detector simulation, which can be seen as another factorised event-generation phase, but is typically provided by the experimental groups as a separate code. After the detector simulation, one can proceed with the event reconstruction as it is done for real data.

A specific advantage over analytic results is that the natural output of the underlying Monte-Carlo integration is fully exclusive in final-state particle content and its kinematic configuration. This allows for generating a set of Monte-Carlo events once, and analyse this data $a$-posteriori with respect to any observable.

Examples for Monte-Carlo event generators that are general enough to be applicable to most physics studies at colliders such as the LHC are Pythia [50], Herwig $[48,49]$ and SHERPA $[46,47]$. In addition, many more specialised event generators exist.

\subsection{The Sherpa framework}

\subsubsection{Physics modules}

The MCEG modularity paradigm is strictly followed in the SHERPA framework. The central program module, also called SHERPA, steers the event generation by passing the event through different phases. This event is modelled as a series of $n \rightarrow m$ particle amplitudes that are added or modified by each phase in turn.

These phases are mostly independent from each other, with a few compromises being made where the physics model requires some additional cross-talk exceeding the actual event. For example, the increased interplay between hard-process and parton-shower calculations led to a strong integration between the matrix-element and the parton-shower 


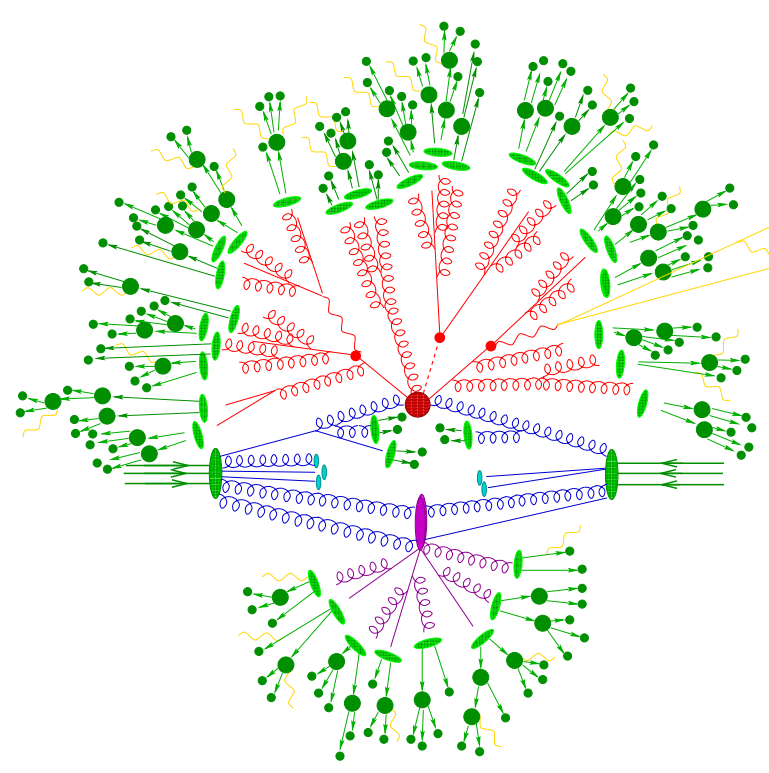

Figure 2.1.: A pictorial representation of a Monte-Carlo event. As an example, the production of a top-antitop quark pair in association with a Higgs-boson is considered. The hard process (large red blob) is followed by the decay of the three particles (small red blobs). QCD bremsstrahlung is produced (red). The incoming partons take part in secondary interactions, which are also dressed by QCD bremsstrahlung (purple). Then the produced partons undergo the fragmentation into hadrons (light green blobs). These possibly decay further into stable hadrons (dark green blobs). During any phase, soft photons can be emitted (yellow).

modules in SHERPA. Otherwise the actual physics implementations are hidden behind abstract event phase interfaces to enforce the modular structure. By this approach, modules can be dynamically chosen by the user, when different implementations of the same event phase are available. In SHERPA, this is for example the case for the jet evolution, where the CSSHOWER [21] and DiRe [69] modules can both be used as the underlying parton shower.

Following the visualisation in Fig. 2.1 from the core to the outer layers, we will now quickly discuss the relevant event phases and their implementation in SHE RPA.

\section{Hard interaction and decays}

The hard-scattering cross section is calculated in SHERPA either by its Comix [70, 71] or its AMEgIC [72] module. The choice can be made per final-state multiplicity, so both can be mixed to combine their strengths.

AMEGIC uses the methods described in $[73,74]$ to generate helicity amplitudes from Feynman diagrams. Its Standard Model results are validated for different multiplicities in [75]. Besides that we also make use of its support for an effective gluon-gluon-Higgs vertex via a top-quark loop, where the latter is integrated out in the infinite top-mass limit. AMEGIC 
automates the Catani-Seymour subtraction method [76] to render separately divergent pieces integrable, as explained in Section 2.2.1. Virtual matrix elements are available from within SHERPA for a few standard processes, and interfaces exist to many one-loop codes. Among those, BlackHat [77], OpenLoops $[78,79]$ and NJet [80] are used in this thesis.

Comix generates tree-level matrix elements using colour-dressed Berends-Giele recursion relations [81]. Also the calculation of phase-space weights is done recursively. This is a key advantage when it comes to high final-state multiplicities, where it is much faster than Amegic.

Two other key components for the hard interaction are shared between AMEGIC and Сомıx, the phase-space integration, and the hard decays of unstable particles in the final state. Both are described in [72], we only point out here that the phase space integrator is based on a multi-channel Monte-Carlo algorithm with VEGAS optimisation [82]. For parts of the final-state integration, implementations based on RAMBO [83], SARGE [84] and HAAG [84] are used.

\section{QCD bremsstrahlung}

The evolution from the hard scale down to the hadronisation scale through the emission of coloured particles is performed by parton-shower algorithms. SHERPA features two implementations, CSSHOWER [21] and Dire [69].

CSSHOWER is the one we use (and reweight) exclusively in this thesis. Its model was proposed in $[85,86]$ and rests on the factorisation of real-emission matrix elements in the Catani-Seymour subtraction scheme [76]. The dipole terms identified in this scheme capture all infrared singularities in general NLO QCD amplitudes. A so-called spectator parton of the splitting takes the recoil, or all final-state partons, if both the splitter and the spectator are initial-state partons. Hence, energy-momentum is conserved at each splitting. Colours are treated in a large- $N_{C}$ limit, where the spectator and the splitter are adjacent in colour space. These notions are explained in Section 2.2.2, when we discuss parton showers in more detail.

The same structure underlies also the S-MC@NLO [87] implementation in SHERPA, which matches fixed-order NLO calculations to the resummation of the parton shower. Moreover, SHER PA also features an implementation of the merging methods in $[30,33,88]$. An account on matching and merging is given in Sections 2.2.3 and 2.2.4, respectively.

\section{QED bremsstrahlung}

The PHOTONS++ [89] module adds higher-order QED corrections both to the hard interaction and to hadron decays. It calculates a resummation of the infrared-singular terms and uses complete first-order corrections for the most relevant cases. The resummation is based on the YFS algorithm [90]. 


\section{Non-perturbative effects: Multiple interactions, hadronisation and hadron decays}

The multiple interactions module AmISIC uses the model presented in [91], and extends it by evolving each additional collision using the CSSHOWER. The organisation of the beam-beam remnants is such that partons adjacent in colour space are also adjacent in momentum space.

When all partons of the hard and multiple interaction are evolved to low energies by the parton showers, the hadronisation module AHADIC translates them to primordial hadrons using the cluster-fragmentation ideas discussed in [92]. An initial implementation of AHADIC is discussed in [93].

The primordial hadrons (and $\tau$ leptons) are then further decayed in HADRONS++, such that one ends with a set of stable particles (i.e. ones that actually enter the detector). The decay products can take into account full spin correlations. The decays are generated partly by matrix elements and by several form-factor models. For $\tau$ leptons, the Kühn-Santamaría model [94] and form-factor parametrisations from Resonance Chiral Theory [95] are used. For hadrons, form factors from heavy-quark effective theory and light-cone sum rules [96] are employed.

Most studies in this thesis stop at the parton-level, i.e. before non-perturbative effects are applied. An exception is the timing study for the reweighting implementation in Section 3.14 .

Many aspects of the hard interaction, the QCD bremsstrahlung and their matching and merging will be discussed from a high-level perspective in Section 2.2, and in quantitative terms in Chapter 3, when we address how to reweight the predictions of these methods. In fact, in terms of SHERPA modules, the implementation of the internal reweighting as detailed in Chapter 3 is mainly concentrated in abstract code shared by AMEGIC and Comix, in the common phase space integrator PHASIC, and in CSSHOWER and S-MC@NLO, safe for some additional handling code in the main steering of SHERPA. We will come back to this at the end of Chapter 3 , when we have explained and validated the reweighting.

\section{Interfaced external codes}

In addition to the already mentioned external one-loop matrix-element providers, a few additional codes interfaced to SHERPA are an essential part of the overall framework for most studies in this thesis. Considering the incoming partons of an event, LHAPDF [97, 98] is often used as the library for the PDF sets, among which we employ NNPDF 3.0 [99], CT10 [100], CT14 [101], MSTW [102] and MMHT2014 [103]. These PDF sets have different methods to encode their uncertainties. The first, NNPDF 3.0, uses a statistical sample, whereas the others use the Hessian method [104] for this purpose. This is reflected in all error bands throughout this work, accordingly. Turning to the output side of the event generation, we generate HePMC [105] event records if events need to be analysed and/or compared to experimental data, for which the RIVET [106] event analysis framework is then 
used. Jets in hadron-hadron collisions are reconstructed with the anti- $k_{T}$ algorithm [107], (mostly) with a radius parameter of $R=0.4$. In $\mathrm{e}^{+} \mathrm{e}^{-}$collisions, the Durham jet algorithm is used instead [108]. For both algorithms, the implementations in the FAstJet package are employed [109]. The notion of jets is introduced in Section 2.2.

\subsubsection{Standard Model choices}

Throughout this work, we use the default choices of SHERPA with respect to the SM. The electro-weak parameters are calculated from the masses of the $\mathrm{W}, \mathrm{Z}$ and $\mathrm{H}$ bosons, and the value of $1 / \alpha_{\mathrm{QED}}$, through tree-level relations. The appearance of unstable fermions or bosons (e.g. the $\mathrm{W}$ or $\mathrm{Z}$ ) as intermediate propagators in the hard process is treated through the complex mass scheme [110], which ensures the gauge-invariance of the matrix elements.

The QCD sector is simplified for the matrix-element calculation by assuming all quarks except for the top quark to be massless. However, for the parton-shower kinematics and for the running of $\alpha_{S}$, finite parton masses are used. Per default, the top-quark mass is considered for the running of $\alpha_{S}$, although in some interpolation-grid studies we use the 5 -flavour scheme instead, to match the $\alpha_{S}$ evolution calculated with the PDF sets, to achieve more precise closure tests. The running is calculated at the perturbative order of the used PDF set and is fixed by setting $\alpha_{S}\left(m_{\mathrm{Z}}^{2}\right)=0.118$, or to neighbouring values in $\alpha_{S}$ uncertainty studies. Another simplification with respect to QCD is the disabling of quark mixing [111], i.e. a unit matrix is used as the скм matrix.

Higgs production via gluon-gluon fusion is calculated with an effective coupling through a top-quark loop in the infinite top-mass limit.

\subsection{The state-of-the-art in perturbative $Q C D$ calculations}

\subsubsection{Pure fixed-order calculations}

In perturbative $\mathrm{QCD}(\mathrm{pQCD})$ calculations, the cross section for a hard-scattering process initiated by the two hadrons $\mathrm{H}_{1}$ and $\mathrm{H}_{2}$ with four momenta $P_{1}$ and $P_{2}$ is given by

$$
\begin{aligned}
\sigma_{\mathrm{H}_{1} \mathrm{H}_{2} \rightarrow \mathrm{X}}=\sum_{i, j} \int \mathrm{d} & x_{1} \mathrm{~d} x_{2} f_{i / \mathrm{H}_{1}}\left(x_{1}, \mu_{F}^{2}\right) f_{j / \mathrm{H}_{2}}\left(x_{2}, \mu_{F}^{2}\right) \\
& \times \hat{\sigma}_{i j \rightarrow \mathrm{X}}\left(x_{1} P_{1}, x_{2} P_{2}, \alpha_{S}\left(\mu_{R}^{2}\right), \frac{Q^{2}}{\mu_{F}^{2}}, \frac{Q^{2}}{\mu_{R}^{2}}\right),
\end{aligned}
$$

with the double sum over all incoming partons $i, j$ that carry momenta $x_{1} P_{1}$ and $x_{2} P_{2}$, respectively. The parton densities within their mother hadrons, $f_{i / H_{1}}$ and $f_{j / H_{2}}$, factorise from the partonic cross section $\hat{\sigma}$, if its characteristic scale $Q$ is much larger than the typical momentum transfers within the hadron.

There are different ways to improve the precision of a $\mathrm{PQCD}$ calculation beyond a fixed LO calculation of a Born matrix element (B) in the strong coupling $\alpha_{S}$. The first step is to 

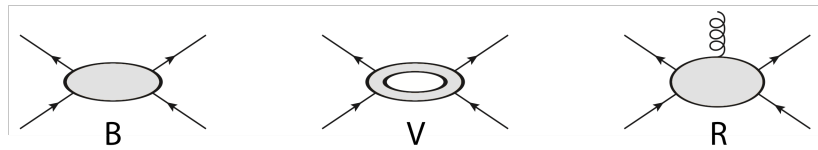

Figure 2.2.: A pictorial representation of the three contributions to a QCD NLO calculation.

include the NLO term in $\alpha_{S}$, by calculating the corresponding Feynman diagrams. If one started with the classical Lagrangian, this makes additional re-orderings necessary. The ultra-violet divergence of loop amplitudes is absorbed into the running of $\alpha_{S}\left(\mu_{R}^{2}\right)$ (and into running quark masses), by resumming logarithmically enhanced terms to all orders of $\alpha_{S}\left(M^{2}\right)$. Here, $M$ is a reference scale, and the ratio $\mu_{R}^{2} / M^{2}$ enters the logarithms. Hence, to add quantum corrections, we need to re-organise the perturbative series. An ordinary Feynman calculation can still be done, if running parameters are used, with a $\mu_{R}$ that is not far from the physical scales of the process. An additional unphysical scale is needed in an NLO calculation, if the initial-state contains hadrons, as in Eq. (2.1). This is the cut-off scale $\mu_{F}$ that separates the non-perturbative low-energy interactions within the hadron from the high-energy process under consideration. Loosely speaking, parton emissions with a transverse momentum $k_{T}^{2}<\mu_{F}^{2}$ are integrated out from the hard interaction, and left to the parametrisation of the hadron structure through its PDFs. By this procedure, the previously purely non-perturbative PDFs acquire a running in $\mu_{F}$. This running is calculable within perturbation theory. The unphysical scales $\mu_{R}$ and $\mu_{F}$ are called the renormalisation and the factorisation scale, respectively.

In addition to the Born part (B), NLO amplitudes comprise virtual loop-corrections (V), where a LO Feynman diagram is combined with a loop diagram (that has two additional powers in $\alpha_{S}$ ), and real radiation (R), for which two diagrams with an additional external parton are combined, see Fig. 2.2. V and R are separately infrared divergent. However, the divergences can be identified by regularisation methods such as dimensional regularisation, and are shown to cancel each other for so-called infrared-safe observables. These observables are defined by the property that additional soft and/or collinear external partons do not change the value of the observable, which makes it necessary to combine "adjacent" partons into jets. However, for a Monte-Carlo integration, the different multiplicities of $\mathrm{V}$ and $\mathrm{R}$ have the consequence, that these parts come in separate events. This separation prevents a direct cancellation, such that the integration is numerically unstable due to the divergences. A solution is to preserve the analytic cancellation of the divergences. This is possible to do in a universal (i.e. observable-independent) way, because of the factorisation of soft- and/or collinear emission kernels from the matrix element. Hence, the details of the full multi-leg matrix-element calculation, for which analytic results would not be readily available, are not necessary for the cancellation. Instead, an approximated matrix element that matches the divergences of the actual matrix element can be used. Its regularised (e.g. by dimensional regularisation) form is then employed for the cancellation, by subtracting the divergences from $\mathrm{R}$ and adding them (integrated analytically over the soft/collinear emission) to $\mathrm{V}$. The 

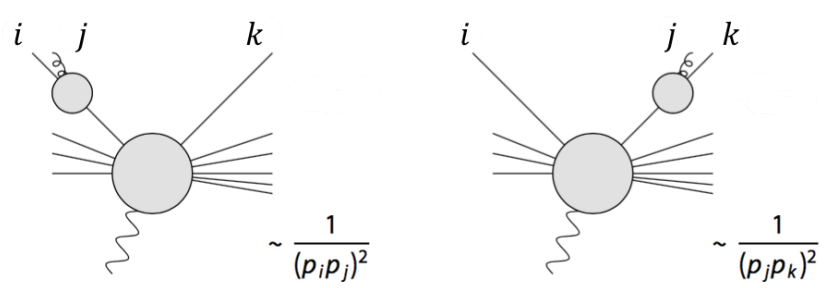

(a) collinear emissions

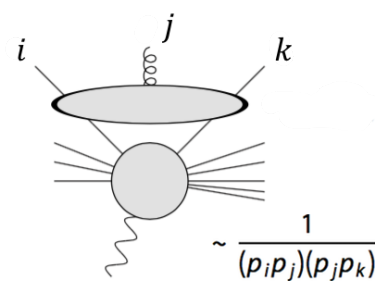

(b) soft gluon emission

Figure 2.3.: Collinear emissions factorise for individual lines, whereas soft emissions factorise only on the level of colour dipoles.

resulting terms are labelled RS (real-subtracted) and VI (virtual-integrated), and are separately finite. ${ }^{1}$ Several such automated subtraction schemes have been formulated (including the analytic results for the integrated subtraction terms), such as the Catani-Seymour (CS) dipole subtraction [76] or the Frixione-Kunszt-Signer (FKS) subtraction [112].

\subsubsection{Exclusively adding log-enhanced emissions - the parton shower}

The fixed-order calculations discussed so far are inclusive with respect to additional QCD radiation below the scale of the hard process. A parton shower is a numerical method to generate explicit emissions from initial- and final-state partons down to a cut-off scale, which is typically chosen to be the scale where the fragmentation of the partons into hadrons should take place. The parton-shower emissions are modelled iteratively and as being independent of each other, i.e. as a Markov Chain. This approximation is based on the soft-collinear divergences of real emissions which render interference effects between different emissions negligible, and on the large- $N_{C}$ limit, whereby colour correlations can be omitted, such that a local colour flow is retained. Within these approximations, real-emission corrections are resummed to all orders (as well as loop corrections, albeit more implicitly via unitarity).

For collinear emissions, the necessary factorisation appears at the cross-section level for each external parton line individually, whereas soft-gluon emissions factorise in this way only on the level of individual amplitudes. At the cross-section level, i.e. after building the squared sum of these amplitudes, these soft factorisations carry over to a sum over all external lines, indicating non-negligible interferences. Hence, soft-gluon emissions have to be interpreted as being emitted by the entirety of the external partons. This phenomenon is called colour coherence [20]. The different factorisation properties are illustrated in Fig. 2.3. Colour coherence makes no difference at the order of the leading logarithms associated with cutting the soft-collinear divergences, but needs to be taken into account to achieve next-to-leading logarithmic accuracy. One way to do it, is to enforce an angular ordering of

${ }^{1}$ In Section 3.1.2, we will also discuss the KP terms. These are the remainders of the cancellation of collinear divergences, that arise when integrating emissions involving initial-state partons from within a hadron. The poles are subtracted into the PDF of the hadron, which can also be done in a universal way owing to the universality of the PDFs. The KP terms are dependent on the factorisation scheme and $\mu_{F}$. 
the emissions, because soft wide-angle gluon emissions from a more collinear parton pair are suppressed. Then, all branchings can still be simulated as coming from single mother partons. This is proven to reproduce the emission patterns of colour coherence. For this reason, parton showers that use an angular ordering are called coherence-improved parton showers.

Another way to heed colour coherence and at the same time keep using the usual ordering in transverse momentum (or a derivative of it), is to simulate emissions as coming from a colour dipole, instead of a single mother parton. This rests on the fact that for large $N_{C}$ a definite colour flow can be defined [113-116]. In fact, such a dipole decomposition for real emissions is used in NLO subtraction schemes such as the CS dipole subtraction, where each dipole is constructed from an emitter and a spectator. This observation led to the development of dipole parton showers based on such subtraction schemes [21-23].

The advantage of dipole showers with transverse momentum evolution over coherenceimproved showers is that by the $p_{T}$ ordering the hardest emission is generated first and is guaranteed not to exceed the hard scale (or intermediate pre-set emission scales from multi-jet merging algorithms). Also, the cut-off scale is more physically meaningful when defined in $p_{T}$ (usually around $1 \mathrm{GeV}$ ), as a resolution criterion of emissions. Also note that the input scale of the strong coupling must be chosen to be of the order of the transverse momentum, to resum some of the higher-order corrections to the branching [117]. Hence, the ordering in transverse momentum, together with such a cut-off choice, ensures that the strong coupling is not evaluated near its Landau pole at $\Lambda_{\mathrm{QCD}} \approx 500 \mathrm{MeV}$. In particular, dipole showers based on NLO dipole subtraction schemes are more easily matched with the hard-scattering cross sections calculated with the same scheme.

The starting conditions of a parton shower are unambiguous for $\mathrm{LO}$ calculations with a single dipole in the final state, such as boson or di-jet production. We speak of a Leading Order matrix elements matched with Parton-Shower emissions (LOPS) event generation then. However, if we include additional parton emissions, either directly by requiring additional jets in the process definition, or by using an NLO calculation (or both), the combination of matrix elements with parton showers becomes less straightforward. These cases will be discussed in the following.

\subsubsection{Matching NLO calculations with a parton shower}

Similarly to the LOPS case, we want to specify a procedure to generate Next-to-Leading Order matrix elements matched with Parton-Shower emissions (NLOPS) events. Here, we have to address the double-counting of terms that are present in both the fixed-order NLO calculation and the LOPS calculation. Several methods have been developed to cure this.

Their common goals are to retain NLO accuracy in the distributions when expanding the method in $\alpha_{S}$, and that at the same time the logarithmic accuracy of the parton shower is preserved. Furthermore, it is desirable that the transition between hard and soft/collinear emission regions is smooth.

The two methods that achieve this are POWHEG [25] and MC@NLO [24]. Mixed schemes 
have also been discussed for a better comparison of the two [87]. In POWHEG, a one-step parton shower is used with the ratio of the real-emission matrix element over the Born matrix element, $\mathrm{R} / \mathrm{B}$, as the parton-shower splitting kernels. The issue with this is, that hard real-emission configurations are exponentiated, which amounts to extending the resummation beyond its region of validity. In addition, not all $\mathrm{R}$ contributions are given by the Born configuration plus one additional parton emission [87].

The latter two problems are not present in the MC@NLO method. It modifies the NLO subtraction, by first splitting off the hard infrared-regular part from R. Hence, only the remaining soft infrared-singular part is exponentiated. In the original MC@NLO, the soft part is defined by the parton-shower kernels. However, these might only be accurate to leading-logarithmic order, and within the approximation from the large- $N_{C}$ limit. Divergences in sub-leading colour configuration would then remain in the subtracted hard part of R. One can fix this by leaving the full NLO subtraction in place, and instead re-defining the parton-shower kernels from the full NLO subtraction terms [87]. This variant is called the S-MC@NLO [87] method. It guarantees, that the one-step shower takes into account all information of the $\mathrm{R}$ matrix element in the soft-collinear limit. The ordinary shower then follows the first step, or in the case of the hard part is directly applied to the matrix element result. This ordinary shower falls back to the large- $N_{C}$ approximation and uses the subtraction kernels in their spin-averaged form. A parton-shower that has been defined in this way is the CSSHower [21] used as the default in SHERPA.

\subsubsection{Merging multiple jet multiplicities}

A multi-jet merged event generation allows to combine LO or NLO QCD matrix elements of arbitrary multiplicities with a parton-shower. The merging procedure solves the problem of double counting and ambiguous starting conditions for higher-multiplicity matrixelements, and at the same time allows for the combination of several matrix-element multiplicities into a single inclusive sample. Accordingly, the production of jets associating a given core process can be modelled through exact matrix elements rather than relying on the logarithmic approximation of the parton shower only, see Fig. 2.4. In particular, when considering hard jet kinematics or angular correlations such techniques prove to be indispensable to properly describe experimental observations, see for instance $[59,61,118,119]$. Compared to NLOPS, a merging calculation offers a better description of the production and kinematics of hard jet emissions, and has proven to be more stable with respect to the choice of the used scheme, even for more inclusive observables such as the Higgs-boson $p_{T}$ distribution [120, 121].

The approaches for performing a multi-jet merged calculation at tree-level are called CKKW [26, 29], CKKW-L [27, 122] and MLM [28]. They have been generalised to NLO in [33, $88,123]$. The LO and NLO merging techniques employed within the SHER PA framework are presented in [30] and [33, 88], respectively, and are a generalisation of the CKKW approach. They rely on the reconstruction of parton-shower histories for multi-parton amplitudes that set the parton-shower initial conditions for their subsequent evolution. This is achieved 


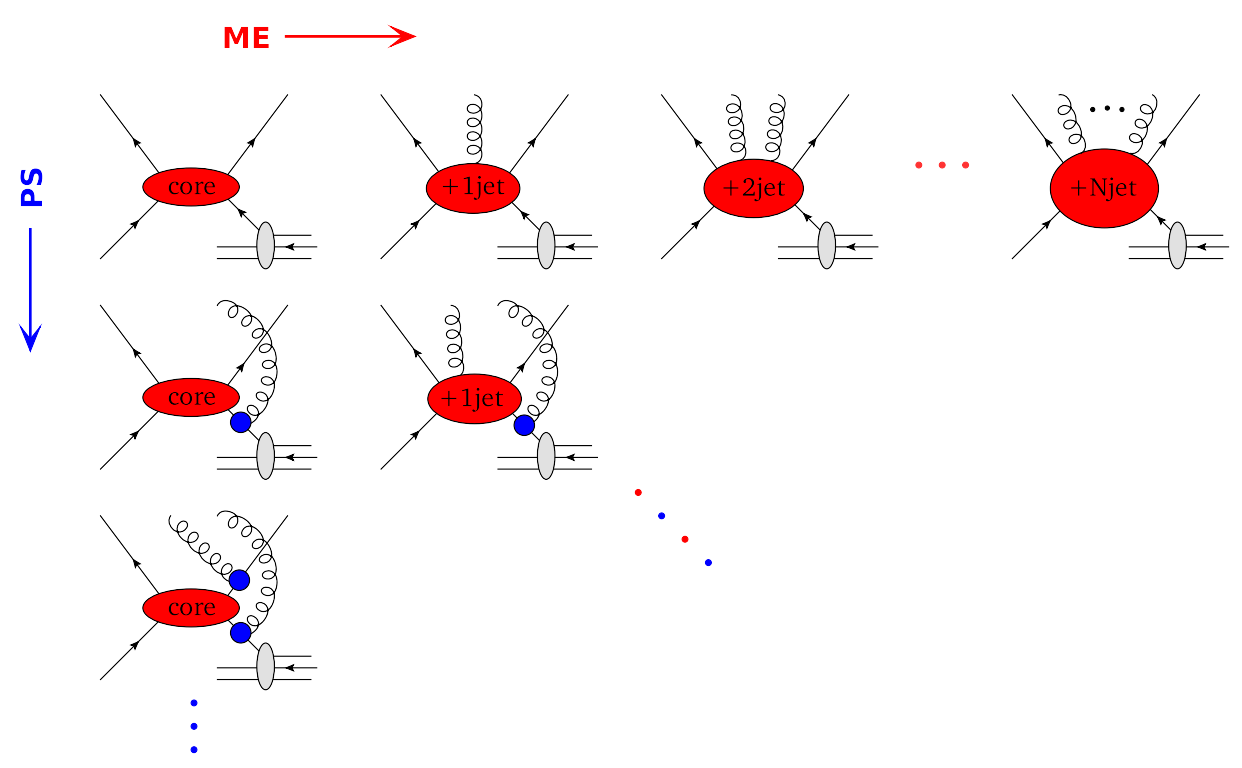

Figure 2.4.: A pictorial representation of the multiplicities generated in a multi-jet merged calculation for a core process (upper left diagram) plus matrix elements for up to $N$ jets (leftright) and additional parton-shower emissions (top-down).

by running a backward-clustering algorithm that identifies a corresponding core process and calculates hard-parton splitting scales that serve as predetermined shower branchings. In the SHERPA approach the actual parton shower then starts off the reconstructed core process while incorporating the predetermined hard splittings based on a truncated shower. This means that the internal lines between the predetermined shower branchings are fed into the parton-shower with the scale of the lower predetermined branching as the cutoff scale. Another scale is used as a phase-space slicing scale. It is called the merging scale $Q_{\text {cut }}$. Matrix element emissions are vetoed below $Q_{\text {cut }}$. Vice versa, parton-shower emissions at scales larger than $Q_{\text {cut }}$ also lead to an event veto ("Sudakov veto"). This is equivalent to a multiplication of all lines with the corresponding no-branching probability, and ensures that the jet multiplicity given by the matrix element calculation is rendered exclusive. This removes the double counting that would occur in a naive sum of matrix elements with different multiplicities. Moreover, by the combination of the vetoed shower and the Sudakov vetoes of the matrix elements their individual dependences on $Q_{\text {cut }}$ cancel out.

An additional consequence of the multi-jet merging technique is the appearance of a natural dynamical scale choice for $\mu_{R}$ and $\mu_{F}$. Instead of fixing their values with respect to the whole multi-leg matrix element, it is done only for the core process. The additional strong couplings and the PDF ratios at the branchings from the backwards-clustering is then chosen as it would have been for ordinary shower branchings for consistency, i.e. as some factor times the transverse momentum of the emitted parton. In particular for the strong 
couplings, this means that we profit from the improved resummation of the higher-order corrections, as has been noted in Section 2.2.2. By not using a single predetermined scale for the multi-leg matrix element, we can thus better accommodate the inherently multi-scale nature of a high-multiplicity matrix element.

Multi-jet merged calculations combining LO or NLO matrix elements and the parton shower are labelled Matrix Elements merged with Parton Shower emissions at Leading Order (MEPS@LO) or MEPS@NLO, respectively. A combination of both, combining one or more multiplicities given at NLO with additional multiplicities given at LO, has also been developed and is referred to as MENLOPS [33, 88, 124-126]. The reason for using this combination is that tree-level matrix elements are available for higher multiplicities than matrix-elements that contain a loop. For the latter, up to 6 external legs are state-of-theart [127], which is about a factor of two smaller than what is computationally feasible for tree-level matrix elements [70].

\subsubsection{Towards NNLO}

The field of NNLO calculations is very active. In the last two years, over a dozen of new results have been presented, including fully differential results for di-photon production [12], single-top (+decay) [13] and top-pair production [14], $\mathrm{W}^{+} \mathrm{W}^{-}\left[\ell^{\prime+} v_{\ell^{\prime}} \ell^{-} \bar{v}_{\ell}\right]$ production ${ }^{2}[15]$ and $\mathrm{V}$-boson production in association with a jet $[16,17]$. Of particular interest are processes with large higher-order corrections, such as Higgs-boson production via gluon fusion, or standard candles like Drell-Yan lepton-pair production. For the latter, the scale uncertainties of the $\mathrm{Z}$ transverse momentum can be reduced by the inclusion of the NNLO to 1-2\% [18]. For $Z+j e t s$ production, the scale uncertainties were reduced to $2 \%$, with a $K$ factor of about $4 \%$ [19]. All these results seem to promise that a full automation of NNLO calculations is in reach. However, there are still major obstacles to overcome, e.g. finding solutions to all master integrals being involved and the construction of a fully general subtraction technique. This is especially true when it comes to amplitudes for multiplicities beyond $2 \rightarrow 2$. For an overview on the achievements and the remaining challenges, see e.g. [128].

As for NLO, the availability of NNLO calculations does not automatically mean that they are readily usable for a Monte-Carlo event generation. A fully generalised methodology has not been achieved yet, but first implementations are available for Drell-Yan and Higgs production based on the MINLO-NNLOPS [37-39] and the UN ${ }^{2}$ LOPS [34-36] schemes.

However, these efforts are considered only as first steps towards a more general Next-tonext-to-Leading Order matrix elements matched with Parton-Shower emissions (NNLOPS) matching [36]. And while the generalisation of Monte-Carlo methods to NNLO is still ongoing, a first fixed-order calculation to next-to-next-to-next-to-leading-order $\left(\mathrm{N}^{3} \mathrm{LO}\right)$ has been published [129], for the gluon-fusion Higgs-boson production cross section. It leads

${ }^{2}$ Throughout this thesis, the symbol $\ell$ stands for an electron or a muon. Brackets following a particle denote off-shell production with subsequent decay into the particles inside the brackets. 
to a reduction of the scale uncertainties from $9 \%$ at NNLO down to $3 \%{ }^{3}$ Although this is not a differential calculation yet, the employed methods are claimed to be extendable towards differential distributions in the future, which will eventually require another round of matching/merging efforts.

To conclude, MEPS@NLO/MENLOPS calculations and the not yet fully-automated NNLO plus parton-shower calculations are our best general-purpose descriptions of perturbative QCD processes that are available today.

\section{3. $Q C D$ uncertainties in the context of event generators}

The uncertainties that interest us most in the context of this thesis can be categorised into parametric and perturbative uncertainties. We discuss them in turn, and then also other categories to further confine our scope. Most importantly, we do not consider non-perturbative uncertainties.

The systematics of leading-order parton-shower simulations with HeRwig 7 have recently been discussed in [130]. A comprehensive comparison of various generators is presented in [128]. The impact of PDFs in parton-shower simulations has been discussed in $[131,132]$. For non-experts it is practical to have a bottom-line prescription for estimating the uncertainties of Monte Carlo event generator predictions, which is e.g. given in [133].

\section{Parametric uncertainties}

With parameters we refer to input quantities from measurements, like particle masses, the value for $\alpha_{S}\left(m_{\mathrm{Z}}^{2}\right)$, and PDFs. Usually the latter two are connected, as the $\alpha_{S}\left(m_{\mathrm{Z}}^{2}\right)$ and the PDFs are fitted together, or the PDFs are fitted with a fixed $\alpha_{S}\left(m_{Z}^{2}\right)$. Therefore, whenever we vary $\alpha_{S}\left(m_{\mathrm{Z}}^{2}\right)$ in this thesis, the use of an associated PDF set is understood. Consistent $\alpha_{S}\left(m_{\mathrm{Z}}^{2}\right) / \mathrm{PDF}$ variations are expected to be less severe than $\alpha_{S}\left(m_{\mathrm{Z}}^{2}\right)$ variations alone, as the PDF values will compensate the lower/higher $\alpha_{S}\left(m_{Z}^{2}\right)$ to some degree. This should at least be true for the observables, for which data points are included in the PDF fit.

In this thesis, we do PDF and $\alpha_{S}\left(m_{Z}^{2}\right) / \mathrm{PDF}$ variations, which are both relevant for partonlevel predictions at low and high energy scales. Therefore, they can not simply be absorbed into tunes of non-perturbative models (see below). On the other hand, we do not vary quark masses, although they clearly are QCD parameters. However, the very light quarks $(\mathrm{u}, \mathrm{d}, \mathrm{s})$ have masses that are smaller than $\Lambda_{\mathrm{QCD}} \approx 500 \mathrm{MeV}$. The c quark mass is only slightly above the usual cut-off scale for the parton shower and its variation is therefore subject to an absorption through non-perturbative tunes; and the $b$ and the $t$ quark mass uncertainties are below $1 \%$, such that they are considerably smaller than the uncertainties we do consider.

${ }^{3}$ Higgs production via gluon fusion is a notorious counter-example for the rule of thumb, that pQCD calculations at order $p$ have an approximate uncertainty of $\alpha_{S}^{p}$. This is due to large colour factors entering the loop corrections. 


\section{Perturbative uncertainties}

This category refers to the approximation through the truncation of the perturbative series, either by the maximum order in $\alpha_{S}$, or by the maximum power of resummed logarithmically enhanced terms. Also of a perturbative nature is the large- $N_{C}$ approximation, as it restricts the contributing Feynman diagrams to planar ones [134].

The perturbative uncertainties are conventionally estimated by varying unphysical scales which are connected to the truncation, to assess the stability of the result under these artificial choices. For the $\alpha_{S}$ truncation, that would be the renormalisation scale $\mu_{R}$ and the factorisation scale $\mu_{F}$. The variation of these two scales is considered throughout this work.

\section{Other uncertainties}

Other uncertainties can be labelled numerical, algorithmic or non-perturbative. Numerical uncertainties can be trivially overcome by investing more resources. The statistical uncertainty can be reduced by generating more events, and the computational precision can be improved e.g. by going from double to quad precision whenever necessary.

Algorithmic uncertainties come from choices in the implementation, like the non-singular terms in parton-shower evolution kernels, its recoil scheme, or the specifics of the matching/merging algorithms. Per construction, for sensible choices, these systematics also correspond to higher-order perturbative corrections, but might be addressed separately, e.g. by comparing the results of different implementations/choices. Some attempts are done in [130].

Lastly, non-perturbative uncertainties appear when using hadronisation and multipleinteraction models. These are phenomenological models with parameters fitted to experimental data, which is called "tuning", see for instance $[135,136] .{ }^{4}$ The parton-shower cut-off scale has a special role. It marks the (to some degree arbitrary) transition scale to the nonperturbative regime. Its choice affects the fit results for the parameters, and therefore its independent variation is not meaningful. As such, it is not a purely perturbative parameter, but induces power corrections of the order of the ratio to the hard scale [134].

By making the multitude of theoretical uncertainties explicit, it becomes clearer that their estimation through dedicated calculations of all associated variations, although desirable, is unfeasible for most applications.

\footnotetext{
${ }^{4}$ For some event generators, tuning extends to parameters controlling certain characteristics of parton-shower emissions, which evolves down to the hadronisation scale and can therefore not be separated cleanly from the soft physics. This is in particular true for parton-shower emissions applied to multiple-interaction remnants. However, in this case the parton-shower emissions from the remnants can be treated differently from the parton-shower emissions from the hard interaction, as is e.g. done in SHERPA.
} 


\subsection{Conclusive closure tests}

In order to validate the reweighting methods presented in this thesis, we conduct closure tests for a given observable $O$. For these, we start with an event generation run with some nominal parameter and scale choices $A$. The resulting prediction $O_{A}$ for $A$ is then reweighted to a prediction $O_{A \rightarrow B}$ for another set of parameters and scale choices $B$. This is then compared with a prediction $O_{B}$ from a second event generation run, where $B$ is used as the nominal parameters and scale choices. Ideally, $O_{A \rightarrow B}=O_{B}$. However, this is usually spoiled by numerical and statistical differences between $O_{A \rightarrow B}$ and $O_{B}$. If the reweighting method includes interpolation, the finite interpolation accuracy is also a source of deviations.

We call the event generation run for $A$ the reweighting run and the one for $B$ the dedicated run.

\subsubsection{Controlling Monte-Carlo statistics}

The outcome of a Monte Carlo event generation depends on the underlying sequence of random numbers. If we use the same random number generator and the same random seed when doing repeated runs, we can ensure that this sequence is the same among these runs. However, this does not guarantee that two runs with differing parameter and scale choices are statistically equivalent. The problem is that the count of random numbers used for a given event may depend on the parameters and scales. A prominent example is the parton shower, which is a Markov chain of variable length: Higher emission probabilities lead to a higher number of branchings, and thus to a higher "use" of random numbers.

This problem can be ameliorated by setting the random seed after each event to a predefined value, such that these variable amounts of random number usages only affect the statistics of one event, but not the statistics of all forthcoming ones. This guarantees at least that the first phase of the event generation, the selection of the hard process channel and its momenta, is always the same. Less events are then needed to reach a given validation target accuracy.

The hard process channel determines the incoming partons, the final-state multiplicity and the part of the calculation (Born, Virtual, ...) of an event. In SHerpa, an optimisation phase calculates the total cross sections of these channels. Their relative sizes then give the probability for the channel to be selected for an event. Moreover, the optimisation determines the distribution used to sample the phase space. Collectively, we call the discrete channel selection probabilities and the phase space distributions the selection weights of the event generation. These weights depend on the parameters and scale choices used for this precursor run. E.g. the outcome for the partonic channel selection for hadronic event generations are affected by the PDF set used. We therefore use the same set of channel selection weights in the reweighting and the dedicated runs if possible. This ensures that the same channel and phase space point is used for a given randon number. ${ }^{5}$

${ }^{5}$ To ensure statistical equivalence between SHERPA runs, it is in fact vital to do the optimisation run separately 
In fact, for a weighted event generation, using the "wrong" selection weights to ensure statistical equivalence will only affect the speed of the statistical convergence. Otherwise, results will be correct and can therefore be used to validate reweighting results with. However, for unweighted events, the selection weights are used for unweighting events. Therefore, we can not reuse the same selection weights, and hence it can not be guaranteed that the same channel is selected for a given event across reweighting and dedicated runs. Also, for most calculations many events are rejected, and the probability for rejection depends on parameter/scale choices. Therefore, each event actually consists of a tower of trial events, of which only the last one is accepted. As this tower is of varying length, the event selection and statistics will only be weakly correlated across reweighting/dedicated runs. In general, it is therefore not possible to validate the reweighting for unweighted events with small statistics runs.

\subsubsection{Basic validations with auto-closure tests}

For auto-closure tests, the nominal set of parameter and scale choices of the reweighting run is equal to the one being reweighted to, i.e. $A=B$ and thus we test how well the ideal equality $O_{A \rightarrow A}=O_{A}$ is fulfilled.

Discrete parameter-dependent random choices in both the reweighting and the dedicated event generation are then always the same (as long as the random number sequence is the same). This means for example, that the parton-shower algorithm will not lead to different kinematics between the event generation runs.

Therefore, it is possible to widen the class of event generations for which the comparison decouples from statistics entirely, and even unweighted and/or parton-showered event generation is expected to give perfect closure between reweighting and dedicated runs. Hence, auto-closure tests are a first "cheap" test, to check for severe errors in the implementation.

\subsubsection{Cross-closure tests and uncertainty band comparisons}

For uncertainty estimates, we combine many parameter variations into a few uncertainty bands. The predictions from PDF error sets are combined to a PDF uncertainty of the observable, those for different $\alpha_{S}\left(m_{\mathrm{Z}}^{2}\right)$ are combined to an $\alpha_{S}$ uncertainty, and those for different scale choices to a scale uncertainty. We employ this for some of our validations, by comparing uncertainty bands generated with our reweighting methods from a single nominal event generation with uncertainty bands generated from dedicated calculations.

Deviations for single lines between the reweighting and the dedicated prediction might be hidden when comparing uncertainty bands. On the positive side, the condensation of information into bands allows for a quick overview, if the methodology works, and is still sensitive to the level of precision that is actually required in actual applications.

from any of the production runs, because the event generator uses the same random number generator for optimisation and production events, without resetting it after the optimisation has finished. 
Table 2.1.: An example variations set-up with two variants with respect to the PDF set choice, used as the default for studies in this thesis. The set-up includes a total of 66 (CT14nlo) or 110 (NNPDF 3.0) parameter variations. Note that each value for $\alpha_{S}\left(m_{\mathrm{Z}}^{2}\right)$ implies that it is used with its associated PDF set variant.

\begin{tabular}{lccc}
\hline & nominal & variations & error band \\
\hline PDF sets & CT14NLO & 56 Hessian error sets with a 90 \% CL & Hessian \\
& NNPDF 3.0 & 100 statistical replicas with a 68 \% CL & statistical \\
$\alpha_{S}\left(m_{\mathrm{Z}}^{2}\right)$ value & 0.118 & $0.115,0.117,0.119,0.121$ & envelope \\
$\mu_{R} / \mu_{F}$ factors & $(1,1)$ & $(1 / 2,1 / 2),(1,1 / 2),(1 / 2,1),(2,1),(1,2),(2,2)$ & envelope \\
\hline
\end{tabular}

In Table 2.1, we present a set of variations, that is employed for uncertainty band validations throughout Chapters 3 and 4 . As the nominal PDF set, CT14NLO is used, and the error band is defined as the combination over its 56 Hessian error sets at a $90 \%$ confidence level. A variant we sometimes employ instead is the NNPDF 3.0 set, for which the error band is defined as the standard deviation over its 100 statistical error sets, that are given at a $68 \%$ confidence level. For $\alpha_{S}\left(m_{\mathrm{Z}}^{2}\right)$, a nominal value of 0.118 is used, and the variations are 0.115 , $0.117,0.119$ and 0.121 . Note that this variation of $\alpha_{S}$ also implies using a PDF variant that has been fitted with this $\alpha_{S}\left(m_{\mathrm{Z}}^{2}\right)$ fixed. This is expected to extenuate the effect of the $\alpha_{S}$ variation in most cases, as the PDF of the varied $\alpha_{S}$ is still fitted to describe the same data as the PDF of the nominal $\alpha_{S}$. This consistent $\alpha_{S}+\mathrm{PDF}$ variation is also part of the PDF 4 LHC recommendations for LHC Run 2 [133]. The envelope of these $\alpha_{S}+$ PDF variations is taken as the respective uncertainty. For the scales we employ a 7-point variation, scaling both $\mu_{R}$ and $\mu_{F}$ down and up by a factor of two, independently, but leaving out crossed variations where one scale is scaled up and the other one is scaled down. The scale uncertainty band is defined as the envelope over the all scale variations. 


\section{Reweighting (N)LO matrix elements and parton showers}

We present a comprehensive treatment to fully trace the $\alpha_{S}$ and PDF dependences in the matrix-element and parton-shower components of particle-level SHERPA [46, 47] simulations in leading-order ( $\mathrm{LO}$ ) and next-to-leading-order (NLO) multi-jet merged calculations [30, 125], based on the SHERPA dipole-shower implementation [21]. Furthermore, we provide the means to quickly evaluate the renormalisation- and factorisation-scale dependence of the fixed-order matrix-element contributions, and to vary the associated PDF and $\alpha_{S}$ input scales within the parton shower.

Our treatment is implemented in the new internal reweighting feature in SHERPA. The approach is based on an event-wise reweighting and allows us to provide with a single generator run a set of variational event weights corresponding to the predefined parameter and scale variations, that would otherwise have to be determined through dedicated reevaluations. The alternative event weights can either be accessed through the output of a HEPMC event record [105], or directly passed via the internal interface of SHERPA to the RIVET analysis framework [106].

The event-wise nature of the internal reweighting is shared with approaches that postpone the re-evaluation until after the event generation, operating on a set of independent weights in the event record, called NTU PLEs. This approach is detailed in $[45,137]$ for NLO calculations. The advantage of this approach is that one does not need to repeat the event generation when new variations become necessary. However, the storage needs for a smooth NLO sample can be of the order of a Terabyte, and NNLO calculations might exceed this by an order of magnitude $[128,138]$. An exact reweighting of the parton shower, as detailed in this section, would require storing even more information. The internal reweighting does not suffer from these practical constraints, as it can act on all available information while each calculation is performed.

The new feature is partly available in the 2.2.1 release, with which it is being used by current Monte-Carlo event productions of the ATLAS experiment, namely for $\mathrm{V}+$ jets samples. It will be fully ready in the upcoming 2.3 release. Other internal reweighting implementations have recently been presented for Herwig 7 [48, 49] and for Py t Hia 8 [50], in [139] and [140], respectively. Both approaches are currently restricted to LO plus parton-shower calculations, and do not extend to matched or merged calculations. A parton-shower-only implementation is also available for the VinCia [116] shower model in [141].

Parts of the contents of this chapter are published in $[128,142]$. 


\subsection{Fixed-order calculations}

In order to re-evaluate a QCD cross-section calculation for a new choice of input parameters, i.e. $\alpha_{S}$, PDFs or renormalisation and factorisation scales, it is necessary to understand and trace-out its respective dependences. This is a rather easy task at LO but is already more involved when considering NLO calculations in a given subtraction scheme. It has been done for the Catani-Seymour (CS) scheme in [45], and for the Frixione-Kunszt-Signer (FKS) one in [137].

In this section, we briefly review the dependence structure and discuss the corresponding reweighting equations for LO and CS-subtracted NLO calculations within the SHERPA framework. With this paragraph we also introduce the notation used in the later sections, which explore the reweighting of more intricate QCD calculations, involving QCD parton showers and merging different final-state multiplicity processes.

\subsubsection{The leading-order case}

A LO parton-level calculation of some observable or measurement function $O$ of the finalstate $\Phi$ is based on Born matrix elements $\mathrm{B}$ of $\mathcal{O}\left(\alpha_{S}^{n}\right)$. It exhibits explicit dependences on the PDFs $f=f_{a}\left(x, \mu_{F}^{2}\right)$, the running strong coupling $\alpha_{S}=\alpha_{S}\left(\mu_{F}^{2}\right)$, the renormalisation scale $\mu_{R}$ and the factorisation scale $\mu_{F}$ :

$$
\langle O\rangle^{\mathrm{LO}}=\int \mathrm{d} \Phi_{B} \mathrm{~B}\left(\Phi_{B}\right) O\left(\Phi_{B}\right)=\lim _{N \rightarrow \infty} \frac{1}{N_{\text {trials }}} \sum_{i=1}^{N} \mathrm{~B}\left(\Phi_{B, i}\right) O\left(\Phi_{B, i}\right),
$$

with $N_{\text {trials }}=\sum_{i=1}^{N} n_{\text {trials }, i}$, the sum over the number of attempts $n_{\text {trials }, i}$ to generate an accepted event configuration for each event $i$, and

$$
\mathrm{B}\left(\Phi_{B}\right)=\mathrm{B}\left(\Phi_{B} ; \alpha_{S}, f ; \mu_{R}, \mu_{F}\right)=\alpha_{S}^{n}\left(\mu_{R}^{2}\right) f_{a}\left(x_{a}, \mu_{F}^{2}\right) f_{b}\left(x_{b}, \mu_{F}^{2}\right) \mathrm{B}^{\prime}\left(\Phi_{B}\right) .
$$

Therein, $B$ is the Born matrix element containing all couplings, symmetry and flux factors, and PDFs, whereas $\mathrm{B}^{\prime}$ has the PDFs, here for assumed two incoming parton flavours $a$ and $b$, and the strong coupling stripped off. Note that we have suppressed the event index $i$ here. It is understood that $\mathrm{B}$ depends on the event kinematics and that $\mu_{R}$ and $\mu_{F}$ can be chosen dynamically, i.e. depending on the momenta (and the flavours). Changing the input parameters $\mu_{R} \rightarrow \tilde{\mu}_{R}, \mu_{F} \rightarrow \tilde{\mu}_{F}$, and the input function $f \rightarrow \tilde{f}, \alpha_{S} \rightarrow \tilde{\alpha}_{S}$ results in

$$
\mathrm{B}\left(\Phi_{B} ; \tilde{\alpha}_{S}, \tilde{f} ; \tilde{\mu}_{R}, \tilde{\mu}_{F}\right)=\tilde{\alpha}_{S}^{n}\left(\tilde{\mu}_{R}^{2}\right) \tilde{f}_{a}\left(x_{a}, \tilde{\mu}_{F}^{2}\right) \tilde{f}_{b}\left(x_{b}, \tilde{\mu}_{F}^{2}\right) \mathrm{B}^{\prime}\left(\Phi_{B}\right) .
$$

From Eq. (3.3) we conclude that for PDF reweighting it is necessary to know the $x_{a, b}$ values of the event.

For an unweighted event generation, the event weights are initially distributed uniformly, 
i.e. $\mathrm{B}\left(\Phi_{B} ; \alpha_{S}, f ; \mu_{R}, \mu_{F}\right)=w_{\text {norm }}$. Equation (3.1) thus simplifies to

$$
\langle O\rangle^{\mathrm{LO}}=\lim _{N \rightarrow \infty} \frac{w_{\text {norm }}}{N_{\text {trials }}} \sum_{i=1}^{N} O\left(\Phi_{B, i}\right) .
$$

Scale and parameter variations work the same way as for weighted events. The only caveat is that applying Eq. (3.3) leads to a non-uniform weight distribution and one has to fall back to Eq. (3.1) for calculating the reweighted $\langle O\rangle$. In partially-unweighted event generations the weight distribution is bounded from above and below. Again, the reweighting can be employed, one only has to expect a broadened weight distribution after the reweighting, with some event weights exceeding those bounds.

\subsubsection{The next-to-leading-order case}

A full NLO parton-level calculation including real-emission and one-loop corrections of $\mathcal{O}\left(\alpha_{S}^{n+1}\right)$ based on CS dipole-subtraction, as introduced in Section 2.2.1, has the following structure:

$$
\begin{aligned}
\langle O\rangle^{\mathrm{NLO}}= & \int \mathrm{d} \Phi_{B}\left[\mathrm{~B}\left(\Phi_{B}\right)+\mathrm{VI}\left(\Phi_{B}\right)+\int \mathrm{d} x_{a / b}^{\prime} \operatorname{KP}\left(\Phi_{B}, x_{a / b}^{\prime}\right)\right] O\left(\Phi_{B}\right) \\
& +\int \mathrm{d} \Phi_{R}\left[\mathrm{R}\left(\Phi_{R}\right) O\left(\Phi_{R}\right)-\sum_{j=1}^{\text {dipoles }} \mathrm{D}_{S, j}\left(\Phi_{B} \cdot \Phi_{1}^{j}\right) O\left(\Phi_{B}^{j}\right)\right] \\
= & \lim _{N \rightarrow \infty} \frac{1}{N_{\text {trials }}}\left\{\sum_{i=1}^{N_{B}}\left[\mathrm{~B}\left(\Phi_{B, i}\right)+\operatorname{VI}\left(\Phi_{B, i}\right)+\operatorname{KP}\left(\Phi_{B, i}, x_{a / b}^{\prime}\right)\right] O\left(\Phi_{B, i}\right)\right. \\
& \left.+\sum_{i=1}^{N_{R}}\left[\mathrm{R}\left(\Phi_{R, i}\right) O\left(\Phi_{R, i}\right)-\sum_{j=1}^{\text {dipoles }} \mathrm{D}_{S, j}\left(\Phi_{B, i} \cdot \Phi_{1, i}^{j}\right) O\left(\Phi_{B, i}^{j}\right)\right]\right\},
\end{aligned}
$$

where the new parts have the following dependences,

$$
\begin{aligned}
\operatorname{VI}\left(\Phi_{B}\right) & \equiv \operatorname{VI}\left(\Phi_{B} ; \alpha_{S}, f ; \mu_{R}, \mu_{F}\right), \\
\operatorname{KP}\left(\Phi_{B}, \mathrm{~d} x_{a / b}^{\prime}\right) & \equiv \operatorname{KP}\left(\Phi_{B}, x_{a / b}^{\prime} ; \alpha_{S}, f ; \mu_{R}, \mu_{F}\right), \\
\mathrm{R}\left(\Phi_{R}\right) & \equiv \mathrm{R}\left(\Phi_{R} ; \alpha_{S}, f ; \mu_{R}, \mu_{F}\right), \\
\mathrm{D}_{S, j}\left(\Phi_{B, i} \cdot \Phi_{1}^{j}\right) & \equiv \mathrm{D}_{S, j}\left(\Phi_{B}^{j} \cdot \Phi_{R \mid B}^{j} ; \alpha_{S}, f ; \mu_{R, j}, \mu_{F, j}\right) .
\end{aligned}
$$

Therein, VI combines the renormalised one-loop matrix element with the I-operator of the CS subtraction scheme. This operator gives the flavour-diagonal endpoint contribution of the integrated subtraction terms. VI is thus separately infrared-finite and exhibits a 
common transformation behaviour. Thus, for $\alpha_{S} \rightarrow \tilde{\alpha}_{S}, f \rightarrow \tilde{f}, \mu_{R} \rightarrow \tilde{\mu}_{R}$ and $\mu_{F} \rightarrow \tilde{\mu}_{F}$,

$$
\begin{aligned}
& \operatorname{VI}\left(\Phi_{B} ; \tilde{\alpha}_{S}, \tilde{f} ; \tilde{\mu}_{R}, \tilde{\mu}_{F}\right) \\
& \quad=\tilde{\alpha}_{S}^{n+1}\left(\tilde{\mu}_{R}^{2}\right) \tilde{f}_{a}\left(x_{a}, \tilde{\mu}_{F}^{2}\right) \tilde{f}_{b}\left(x_{b}, \tilde{\mu}_{F}^{2}\right)\left[\mathrm{VI}^{\prime}\left(\Phi_{B}\right)+c_{R}^{\prime(0)} l_{R}+\frac{1}{2} c_{R}^{\prime(1)} l_{R}^{2}\right],
\end{aligned}
$$

with $\alpha_{S^{-}}$and PDF-independent coefficients $c_{R}^{\prime(i)}$ and $l_{R}=\log \left(\tilde{\mu}_{R}^{2} / \mu_{R}^{2}\right)$. Again, VI' is stripped of all coupling and PDF factors.

The KP-terms are defined as the remainders of the integrated dipole-subtraction terms, containing all flavour changing and $x_{a / b}^{\prime}$-dependent pieces, combined with the collinear counter-terms. Here, $x_{a / b}^{\prime}$ are the ratios of the partonic momentum fractions in the respective dipole before and after radiation. Again, this combination is separately infrared-finite and transforms as one unit. When evaluated for the modified set of input parameters, they read

$$
\begin{aligned}
& \mathrm{KP}\left(\Phi_{B}, x_{a / b}^{\prime} ; \tilde{\alpha}_{S}, \tilde{f}_{\tilde{\mu}} \tilde{\mu}_{R}, \tilde{\mu}_{F}\right) \\
& =\tilde{\alpha}_{S}^{n+1}\left(\tilde{\mu}_{R}\right) \tilde{f}_{a}\left(x_{a}, \tilde{\mu}_{F}^{2}\right) \tilde{f}_{b}\left(x_{b}, \tilde{\mu}_{F}^{2}\right) \mathrm{KP}^{\prime} \\
& =\tilde{\alpha}_{S}^{n+1}\left(\tilde{\mu}_{R}\right)\left[\left(\tilde{f}_{a}^{q} c_{F, a}^{\prime(0)}+\tilde{f}_{a}^{q}\left(x_{a}^{\prime}\right) c_{F, a}^{\prime(1)}+\tilde{f}_{a}^{g} c_{F, a}^{\prime(2)}+\tilde{f}_{a}^{g}\left(x_{a}^{\prime}\right) c_{F, a}^{\prime(3)}\right) \tilde{f}_{b}\left(x_{b}, \tilde{\mu}_{F}^{2}\right)\right. \\
& \left.\quad+\tilde{f}_{a}\left(x_{a}, \tilde{\mu}_{F}^{2}\right)\left(\tilde{f}_{b}^{q} c_{F, b}^{\prime(0)}+\tilde{f}_{b}^{q}\left(x_{b}^{\prime}\right) c_{F, b}^{\prime(1)}+\tilde{f}_{b}^{g} c_{F, b}^{\prime(2)}+\tilde{f}_{b}^{g}\left(x_{b}^{\prime}\right) c_{F, b}^{\prime(3)}\right)\right],
\end{aligned}
$$

with the coefficients $c_{F, a / b}^{\prime(i)}=\tilde{c}_{F, a / b}^{(i)}+\bar{c}_{F, a / b}^{(i)} l_{F}$ for $i \in\{0, \ldots, 3\}, l_{F}=\log \left(\tilde{\mu}_{F}^{2} / \mu_{F}^{2}\right)$, and

$$
\begin{array}{rlrl}
\tilde{f}_{q}^{q} & =\tilde{f}_{q}\left(x_{q}, \tilde{\mu}_{F}^{2}\right), & \tilde{f}_{g}^{q} & =\sum_{q} \tilde{f}_{q}\left(x_{g}, \tilde{\mu}_{F}^{2}\right), \\
\tilde{f}_{q}^{q}\left(x_{q}^{\prime}\right) & =x_{q}^{\prime} \tilde{f}_{q}\left(\frac{x_{q}}{x_{q}^{\prime}}, \tilde{\mu}_{F}^{2}\right), & \tilde{f}_{g}^{q}\left(x_{g}^{\prime}\right)=x_{g}^{\prime} \sum_{q} \tilde{f}_{q}\left(\frac{x_{g}}{x_{g}^{\prime}}, \tilde{\mu}_{F}^{2}\right), \\
\tilde{f}_{q}^{g}=\tilde{f}_{g}\left(x_{q}, \tilde{\mu}_{F}^{2}\right), & \tilde{f}_{g}^{g}=\tilde{f}_{g}\left(x_{g}, \tilde{\mu}_{F}^{2}\right), \\
\tilde{f}_{q}^{g}\left(x_{q}^{\prime}\right)=x_{q}^{\prime} \tilde{f}_{g}\left(\frac{x_{q}}{x_{q}^{\prime}}, \tilde{\mu}_{F}^{2}\right), & \tilde{f}_{g}^{g}\left(x_{g}^{\prime}\right)=x_{g}^{\prime} \tilde{f}_{g}\left(\frac{x_{g}}{x_{g}^{\prime}}, \tilde{\mu}_{F}^{2}\right),
\end{array}
$$

for $a, b=\{q, g\}$, respectively. Here, the sum over $q$ includes all light-quark flavours, corresponding to all quarks potentially emitting a gluon. We note that in order to obtain the reweighted expressions for the VI and KP contributions, the additional book-keeping of the $c_{R}^{\prime(i)}, \tilde{c}_{F, a / b}^{(i)}$ and $\bar{c}_{F, a / b}^{(i)}$ (altogether 18) ${ }^{1}$ coefficients is required [45]. Due to its composite structure, the KP-terms do not have a coupling- and PDF-stripped version $\mathrm{KP}^{\prime}$. Nonetheless, we formally introduce a still PDF-dependent version $\mathrm{KP}^{\prime}$ in the second line of Eq. (3.8),

\footnotetext{
${ }^{1}$ The two parameters $c_{R}^{\prime(i)}$ correspond to the single- and double-pole coefficients of the one-loop matrix element while the remaining sixteen coefficients are comprised of eight pairs of coefficients, $\bar{c}_{F, a / b}^{(i)}$ and $\tilde{c}_{F, a / b}^{(i)}$, corresponding to the $\mu_{F}$-dependent and -independent parts for all four flavour structures of each beam, respectively.
} 
for reference in later sections.

The remaining pieces of Eq. (3.5) are the Born matrix element B, the real-emission contribution $\mathrm{R}$ and the differential dipole subtraction terms $\mathrm{D}_{S, j}$. The latter is defined by an underlying Born configuration $\Phi_{B}^{j}$ through its dipole-dependent phase-space map, employing the phase-space factorisation $\Phi_{R}=\Phi_{B} \cdot \Phi_{1}^{j}$. The transformation of B under the exchange of input parameters was detailed in Eq. (3.2), and the transformation of $\mathrm{R}$ and the $\mathrm{D}_{S, j}$ contributions works the same, only with an additional power in $\alpha_{S}$.

\subsection{Closure tests for fixed-order calculations}

The reweighting approach outlined above has been implemented in the SHERPA framework for the two matrix-element generators AMEgIC [72] and Comix [70, 71] in conjunction with the corresponding CS dipole-subtraction implementation [143]. The required decomposition of virtual amplitudes is generic and can be used for matrix elements from BLACKHat [77], OpenLoops [78, 79], GoSam [144], NJet [80], the internal library of simple $2 \rightarrow 2$ processes, or loaded via the BLHA interface [145].

Here we shall present the validation of the reweighting approach of NLO QCD event samples in particular. For that purpose we consider W-boson production in $13 \mathrm{TeV}$ protonproton collisions at NLO QCD, and focus on the transverse-momentum distributions for the $\mathrm{W}$ and the lepton it decays to. In Fig. 3.1, the scale, $\alpha_{S}$ and PDF uncertainty bands for the W $p_{T}$ and the lepton $p_{T}$ distributions are presented. For the bands, we use the prescription of Table 2.1, with the NNPDF 3.0 set. All three bands have been produced for both observables using the internal reweighting of SHERPA from a single event generation run. The nominal scale choice is $\mu_{F}=\mu_{R}=H_{T}^{\prime}$, with

$$
H_{T}^{\prime 2}=\left(m_{T, \ell \bar{v}_{\ell}}+\sum_{i} p_{T, i}\right)^{2} .
$$

In general, the sum runs over all final-state particles except for the lepton-neutrino pair. Hence for NLO W production, the sum has one (real-emission phase space) or zero terms (Born phase space). The lepton-neutrino pair enters with its transverse mass, to take into account the mass scale of the $\mathrm{W}$ resonance. This scale choice has been motivated in [146]. The jet is reconstructed with the anti- $k_{T}$ algorithm [107], with a jet radius parameter $R=0.4$ and a transverse momentum cut of $p_{T}^{\mathrm{j}}>20 \mathrm{GeV}$. The lepton cuts are $\eta^{\ell}<3.5, p_{T}^{\ell}>25 \mathrm{GeV}$ ( or $E_{T}^{\text {miss }}>25 \mathrm{GeV}$ for the $\bar{v}_{\ell}$ ), and the lepton-neutrino pair has to satisfy $60 \mathrm{GeV}<m_{\ell \bar{v}_{\ell}}<$ $100 \mathrm{GeV}$. Here, $\eta$ is the pseudo-rapidity, $E_{T}^{\text {miss }}$ the missing transverse energy, and $m_{\ell \bar{v}_{\ell}}$ the invariant mass of the lepton-neutrino pair.

The running of $\alpha_{S}\left(\mu_{F}^{2}\right)$ is calculated within SHERPA using its renormalisation group equation at NLO with parton thresholds as given by the PDF set. The treatment of partonic thresholds deserves a short discussion. While flavour thresholds in the running of $\alpha_{S}$ do not present any challenges to the reweighting algorithm as $\alpha_{S}\left(\mu^{2}\right)>0$ for all $\mu^{2}>0$ and any loop 
order, this is different for the PDFs, where crossing a parton threshold results in a vanishing PDF for that flavour. Hence, the cross section component of the given partonic channel may be zero if no other non-zero contribution exists. Such an event will be discarded and, thus, cannot be reweighted. If now the respective parton threshold of the target PDF is smaller than the target factorisation scale while the one of the nominal PDF is larger than the nominal factorisation scale we are in a region of phase space where the reweighting must fail to reproduce a dedicated calculation. This could be remedied by storing events as well which vanish solely due to crossing PDF thresholds. However, as only observables sensitive to on-threshold production of light quarks (typically bottom quarks) are susceptible to these effects, they are of little relevance to the vast majority of LHC observables.

Comparing the uncertainties for $p_{T}^{\mathrm{W}}$, we observe that the scale uncertainties are the largest, with relative deviations of $\mathcal{O}(10 \%)$. The relative deviations related to the PDF and the strong coupling do not exceed $\sim 3 \%$. The scale uncertainty exhibits a minimum for $100 \mathrm{GeV}<p_{T}^{\mathrm{W}}<200 \mathrm{GeV}$. The reason is that the variations of $\mu_{F}$ alone cross the central value prediction in this range, such that only the $\mu_{R}$ variation contributes to the overall scale uncertainty here.

Note that $p_{T}^{\mathrm{W}}=0$ at $\mathcal{O}\left(\alpha_{S}^{0}\right)$, and therefore only real-emission events contribute to the distribution. Hence, the observable is only described to LO. We introduce it here as a reference for our later validations including the parton-shower, which use this observable. For the current validation, we complement the discussion of the $\mathrm{W}$-boson transverse momentum with the one of the lepton it decays to. The region below $m_{\mathrm{W}} / 2$ is already filled at $\mathcal{O}\left(\alpha_{S}^{0}\right)$, and therefore we partly have a true NLO description for this observable. In fact, the scale uncertainties are much larger in that region, especially towards the $m_{\mathrm{W}} / 2$ threshold, and at the lepton $p_{T}$ cut at $25 \mathrm{GeV}$. This gives a more realistic picture of the perturbative uncertainties than in the LO region above the threshold.

The small panels on the right of Fig. 3.1 compare the uncertainty bands calculated using the reweighting approach to uncertainty bands where dedicated calculations have been done for each variation. We observe that the bands overlap perfectly for both observables. This is because the reweighting as presented above is exact and for all runs the same phasespace points could be used, as explained in Section 2.4.1. The reweighted and the dedicated predictions for each variation are therefore equal, and so are the uncertainty bands. ${ }^{2}$

\subsection{Parton showers}

\subsubsection{Sudakov form factors}

Parton-shower algorithms are the exclusive counterpart to DGLAP evolution [147] (or its higher-order refinements). As such they use a re-formulation of the DGLAP evolution equations in terms of Sudakov form factors [148], which give the no-emission probability

\footnotetext{
${ }^{2}$ The reweighted and the dedicated calculations are implemented independently, such that their predictions can vary within the numerical uncertainties of the calculation. However, this lies several orders of magnitude below the physical uncertainties considered here, and is not visible in Fig. 3.1.
} 

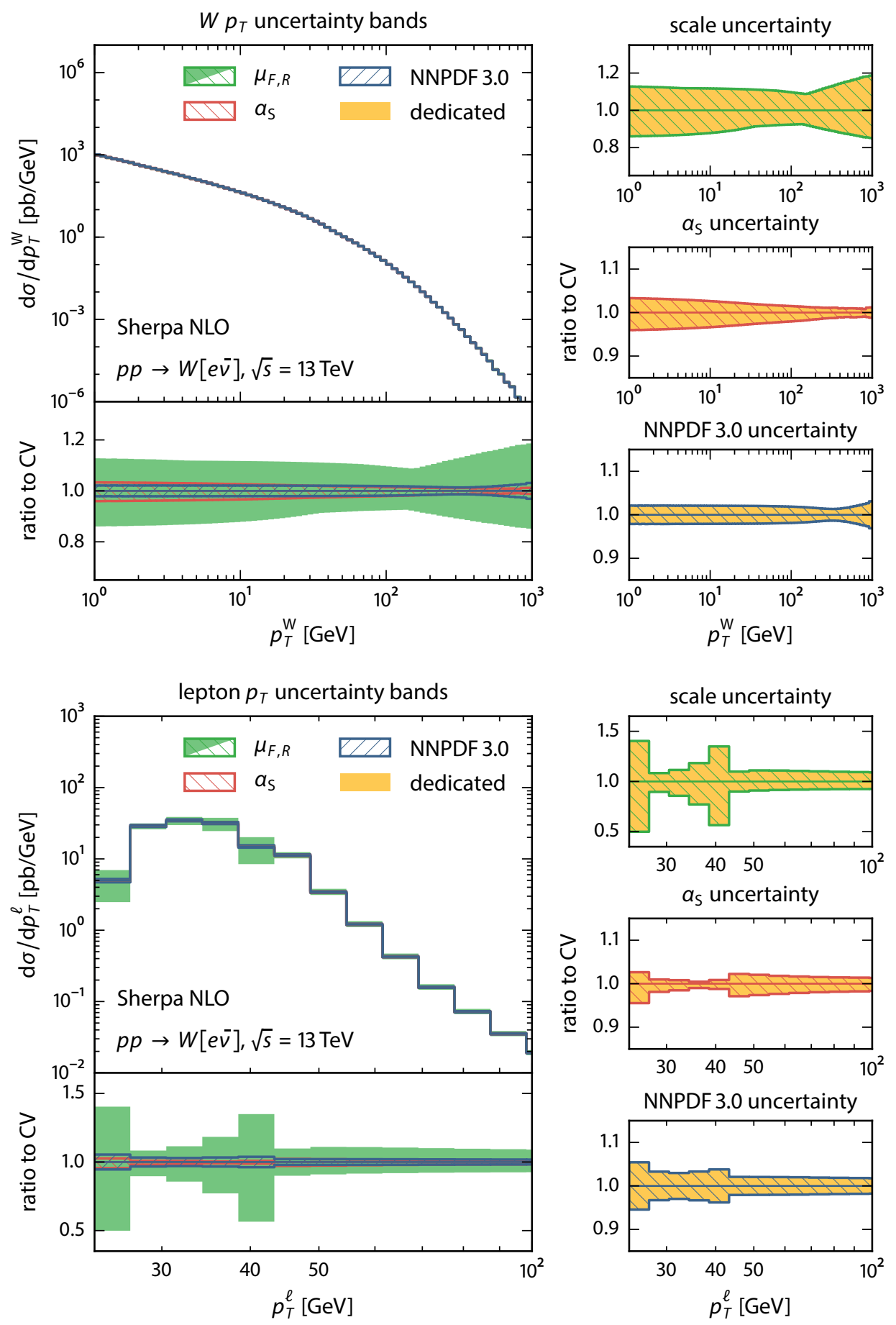

Figure 3.1.: The W-boson and lepton transverse momenta in off-shell W production, with independent variations of $\mu_{R} / \mu_{F}$ (green), $a_{S}$ (red) and the PDF (blue). In the right-hand panels, the individual uncertainty bands, calculated via an internal reweighting, are compared to uncertainty bands from dedicated calculations (yellow). The reweighted and dedicated uncertainty bands are found to be equal. 
for the branching $a \rightarrow b$ between the two evolution scales $t_{1}$ and $t_{2}$,

$$
\Delta_{a b}\left(t_{2}, t_{1}\right)=\exp \left(-\int_{t_{2}}^{t_{1}} \frac{\mathrm{d} t}{t} \int \mathrm{d} z \int \frac{\mathrm{d} \phi}{2 \pi} J(t, z, \phi) \mathrm{K}_{a b}(t, z, \phi)\right) .
$$

The emission kinematics is parametrised by the evolution variable $t$, the portion of the longitudinal momentum fraction inherited from the splitter, $z$, and the azimuthal angle $\phi$. $J$ is the Jacobian associated with this parametrisation, and $K$ is the emission kernel, which in the simplest case is given by

$$
\mathrm{K}_{a b}(t, z, \phi)=\frac{\alpha_{S}(t)}{2 \pi} P_{a b}
$$

with $P_{a b}$ being the DGLAP splitting function, but the exact form depends on the shower model. One important complication is the inclusion of a PDF ratio in $\mathrm{K}$ to guide the backwards-evolution of initial-state showers. We will discuss this in more detail in Section 3.4. For the time being, we can reduce Eq. (3.10) further to a more schematic form,

$$
\Delta\left(t_{2}, t_{1}\right)=\exp \left(-\int_{t_{2}}^{t_{1}} \mathrm{~d} t \mathrm{~K}(t)\right),
$$

where we have absorbed the $z$ and $\phi$ integrals and the Jacobian into the emission kernel, and omit the $a \rightarrow b$ subscript.

\subsubsection{The (reweighted) Sudakov Veto Algorithm}

The advantage of Sudakov form factors is that they can easily be Monte-Carlo integrated by sampling each integral in turn with a random number (and sampling over the possible channels $a \rightarrow b$ ), resulting in a list of concrete emissions. The first task for each emission is to find the next emission scale $t$ below a starting scale $t_{0}$, we can sample the Sudakov form factor with a random number $R$ :

$$
\exp \left(-\int_{t}^{t_{0}} \mathrm{~d} t \mathrm{~K}(t)\right)=R \exp \left(-\int_{0}^{t_{0}} \mathrm{~d} t \mathrm{~K}(t)\right)
$$

If $\mathrm{K}$ is integrable (with the indefinite integral $\mathcal{K}$ ), we can analytically solve for $t$ :

$$
t=\mathcal{K}^{-1}\left(\mathcal{K}\left(t_{0}\right)+\log (1-R)\right) .
$$

However, most physical emission kernels are not integrable analytically and a direct numerical integration of Eq. (3.13) is usually expensive, especially for backwards evolution, where PDFs are part of the kernel. An alternative is an indirect numerical integration, which can be achieved by the Sudakov Veto Algorithm (SVA) [87, 149-153] $]^{3}$. Therein the kernels K are replaced with integrable over-estimates $\hat{\mathrm{K}}$. This is balanced by only accepting a proposed

\footnotetext{
${ }^{3}$ The Sudakov Veto Algorithm is also known as the Thinning Algorithm [154] in computer science, as has been noted in [140].
} 
emission with probability $\mathcal{P}_{\text {acc }}=\mathrm{K} / \hat{\mathrm{K}}$.

An important consequence for the purpose of reweighting is that a multiplicative factor in $\mathrm{K}$ is equivalent to a multiplicative factor in $\mathcal{P}_{\text {acc }}$ [151]. This observation is for example used to apply matrix-element corrections [87], where the emission kernels are replaced with a real-emission-like kernel $\mathrm{R} / \mathrm{B}$. This is done a-posteriori, i.e. the event weight is multiplied by $(\mathrm{R} / \mathrm{B}) / \mathrm{K}$, the emission itself is unchanged.

For convenience, we reproduce the modified (i.e. reweighted) SVA and its proof, in a similar way as in $[151,153]$. The task is to generate emission scales $t_{i}$ for the (nominal) splitting kernel $K$, by using a suitably defined integrable over-estimate $\hat{K} \geq K$. At the same time, we want to use the $t_{i}$ for another (reweighted) emission kernel $\tilde{\mathrm{K}}$, by using weights $w$ to counteract that they have been generated for K. We start with the nominal Sudakov form factor,

$$
\Delta_{\mathrm{K}}\left(t_{2}, t_{1}\right)=\exp \left(-\int_{t_{2}}^{t_{1}} \mathrm{~d} t \mathrm{~K}(t)\right)
$$

To keep the notation clean, we denote with $\mathrm{K}(t), \hat{\mathrm{K}}(t)$ and $\tilde{\mathrm{K}}(t)$ the emission kernels with all kinematic variables except for the emission scale itself integrated out. ${ }^{4}$ The reweighted SVA then reads:

1. Start with $i=0$, a starting scale $t_{0}$ and a weight $w=1$.

2. Add 1 to $i$ and select $t_{i}=\hat{\mathcal{K}}^{-1}\left(\hat{\mathcal{K}}\left(t_{i-1}\right)-\log R\right)$ with the random number $R$, where $\hat{\mathcal{K}}$ is the indefinite integral over the integrable over-estimate $\hat{\mathrm{K}}$. If $t_{i}<t_{\mathrm{IR}}$, where $t_{\mathrm{IR}}$ is the constant (infrared) cut-off scale, the algorithm ends.

3. Draw another random number $R^{\prime}$; if $\mathcal{P}_{\text {acc }}=\mathrm{K}\left(t_{i}\right) / \hat{\mathrm{K}}\left(t_{i}\right) \leq R^{\prime}$, the trial emission is vetoed. In this case, multiply $w$ with

$$
q_{\mathrm{rej}}\left(t_{i}\right) \equiv \frac{\hat{\mathrm{K}}\left(t_{i}\right)-\tilde{\mathrm{K}}\left(t_{i}\right)}{\hat{\mathrm{K}}\left(t_{i}\right)-\mathrm{K}\left(t_{i}\right)}
$$

and return to Item 2 .

4. Otherwise, accept $t_{i}$ as the first emission scale below $t_{0}$ and multiply $w$ with

$$
q_{\mathrm{acc}}\left(t_{i}\right) \equiv \frac{\tilde{\mathrm{K}}\left(t_{i}\right)}{\mathrm{K}\left(t_{i}\right)} .
$$

Hence, for each accepted trial emission following $n$ rejected trial emissions, $w$ gets multiplied by a combined reweighting factor $\mathcal{Q}^{\text {(emission) }}=q_{\text {rej, }, 1} q_{\text {rej, } 2} \ldots q_{\text {rej }, n} q_{\text {acc }}$. After the last accepted emission, additional rejected trial emissions might occur before the cut-off $t_{\mathrm{IR}}$

\footnotetext{
${ }^{4}$ For generalisations of the SVA with respect to a non-trivial $z$ integration and multiple emission channels, see e.g. [155]. These generalisations are not relevant for the reweighting part of the presented proof.
} 
is reached, so we have another reweighting factor $\mathcal{Q}^{\text {(remainder) }}=q_{\mathrm{rej}, 1} q_{\mathrm{rej}, 2} \ldots q_{\mathrm{rej}, n}$ for that region.

To prove that the above algorithm produces the right emission scale distribution for $\tilde{\mathrm{K}}$, when the weight factors $q_{\mathrm{rej}}$ and $q_{\mathrm{acc}}$ are applied, we first consider the probabilities $\mathcal{P}_{n}\left(t_{0}, t\right)$ for accepting $t$ as the first emission scale below $t_{0}$ after $n$ vetoed trial emissions. If no trial emission had been rejected, we obtain

$$
\mathcal{P}_{0}\left(t_{0}, t\right)=\mathcal{P}_{\hat{\mathrm{K}}}\left(t_{0}, t\right) \frac{\mathrm{K}(t)}{\hat{\mathrm{K}}(t)} q_{\mathrm{acc}}(t) .
$$

The first factor on the right-hand side is defined as the probability that the first emission below a starting scale $t_{0}$ is at $t$ when the emission kernel is $\hat{\mathrm{K}}$. This probability is directly connected to the Sudakov form factor, i.e. the no-emission probability between two scales,

$$
\mathcal{P}_{\hat{\mathrm{K}}}\left(t_{0}, t\right)=-\frac{\mathrm{d} \Delta_{\hat{\mathrm{K}}}\left(t_{0}, t\right)}{\mathrm{d} t}=\Delta_{\hat{\mathrm{K}}}\left(t_{0}, t\right) \hat{\mathrm{K}}(t) .
$$

The second factor on the right-hand side of Eq. (3.18) is the probability that the SVA accepts $t$. The third one is the weight we apply for an accepted trial emission. Inserting $\mathcal{P}_{\hat{\mathrm{K}}}$ and $q_{\text {acc }}$ yields

$$
\mathcal{P}_{0}\left(t_{0}, t\right)=\Delta_{\hat{\mathrm{K}}}\left(t_{0}, t\right) \tilde{\mathrm{K}}\left(t_{0}, t\right) .
$$

If exactly one trial emission has been rejected by the SVA at any intermediate scale $t_{1}$, we find

$$
\begin{aligned}
\mathcal{P}_{1}\left(t_{0}, t\right) & =\int_{t_{0}}^{t} \mathrm{~d} t_{1} \mathcal{P}_{\hat{\mathrm{K}}}\left(t_{0}, t_{1}\right)\left(1-\frac{\mathrm{K}\left(t_{1}\right)}{\hat{\mathrm{K}}\left(t_{1}\right)}\right) q_{\mathrm{rej}}\left(t_{1}\right) \mathcal{P}_{\hat{\mathrm{K}}}\left(t_{1}, t\right) \frac{\mathrm{K}(t)}{\hat{\mathrm{K}}(t)} q_{\mathrm{acc}}(t) \\
& =\mathcal{P}_{0}\left(t_{0}, t\right) \int_{t_{0}}^{t} \mathrm{~d} t_{1}\left(\hat{\mathrm{K}}\left(t_{1}\right)-\tilde{\mathrm{K}}\left(t_{1}\right)\right),
\end{aligned}
$$

where we used $\Delta\left(t_{0}, t_{1}\right) \Delta\left(t_{1}, t\right)=\Delta\left(t_{0}, t\right)$, and had to put in the rejection probability $(1-\mathrm{K} / \hat{\mathrm{K}})$ and the rejection weight $q_{\mathrm{rej}}$.

If exactly two trial emissions have been rejected at intermediate scales $t_{1}$ and $t_{2}$, we have

$$
\begin{aligned}
\mathcal{P}_{2}\left(t_{0}, t\right) & =\mathcal{P}_{0}(t) \int_{t_{0}}^{t_{1}} \mathrm{~d} t_{1}\left(\hat{\mathrm{K}}\left(t_{1}\right)-\tilde{\mathrm{K}}\left(t_{1}\right)\right) \int_{t_{1}}^{t} \mathrm{~d} t_{2}\left(\hat{\mathrm{K}}\left(t_{2}\right)-\tilde{\mathrm{K}}\left(t_{2}\right)\right) \\
& =\mathcal{P}_{0}\left(t_{0}, t\right) \frac{1}{2}\left[\int_{t_{0}}^{t} \mathrm{~d} t^{\prime}\left(\hat{\mathrm{K}}\left(t^{\prime}\right)-\tilde{\mathrm{K}}\left(t^{\prime}\right)\right)\right]^{2}
\end{aligned}
$$

where we extend the triangular integration area in the first line to a square one to decouple the integrals. This is balanced by the factor $1 / 2$. The generalisation for $n$ intermediate rejections is

$$
\mathcal{P}_{n}\left(t_{0}, t\right)=\mathcal{P}_{0}\left(t_{0}, t\right) \frac{1}{n !}\left[\int_{t_{0}}^{t} \mathrm{~d} t^{\prime}\left(\hat{\mathrm{K}}\left(t^{\prime}\right)-\tilde{\mathrm{K}}\left(t^{\prime}\right)\right)\right]^{n} .
$$

Because we are not interested in the number of rejections the SVA uses internally, we have 
to sum over this number to get the actual probability distribution for accepting $t$ with the (reweighted) SVA:

$$
\begin{aligned}
\mathcal{P}\left(t_{0}, t\right) & =\sum_{n=0}^{\infty} \mathcal{P}_{n}\left(t_{0}, t\right) \\
& =\mathcal{P}_{0}\left(t_{0}, t\right) \sum_{n=0}^{\infty} \frac{1}{n !}\left[\int_{t_{0}}^{t} \mathrm{~d} t^{\prime}\left(\hat{\mathrm{K}}\left(t^{\prime}\right)-\tilde{\mathrm{K}}\left(t^{\prime}\right)\right)\right]^{n} \\
& =\mathcal{P}_{0}\left(t_{0}, t\right) \exp \left\{\int_{t_{0}}^{t} \mathrm{~d} t^{\prime}\left(\hat{\mathrm{K}}\left(t^{\prime}\right)-\tilde{\mathrm{K}}\left(t^{\prime}\right)\right)\right\} \\
& =\Delta_{\tilde{\mathrm{K}}}\left(t_{0}, t\right) \tilde{\mathrm{K}}(t) \\
& =\mathcal{P}_{\tilde{\mathrm{K}}}\left(t_{0}, t\right),
\end{aligned}
$$

where the last identity finally proves that the distribution for the emission kernel $\tilde{\mathrm{K}}$ is generated by the reweighted SVA.

Note that the weights $q_{\mathrm{rej}}$ and $q_{\mathrm{acc}}$ are exactly the factors by which the acceptance and the veto probabilities are modified, if one would have used $\tilde{K}$ instead of $K$ in the first place. This is obvious for the acceptance weight $q_{\text {acc. }}$. For $q_{\text {rej, }}$, a few lines of algebra are involved,

$$
\begin{aligned}
\mathcal{P}_{\text {rej }}=1-\mathcal{P}_{\text {acc }} \rightarrow 1-q_{\text {acc }} \mathcal{P}_{\text {acc }} & =\frac{1-q_{\text {acc }} \mathcal{P}_{\text {acc }}}{\mathcal{P}_{\text {rej }}} \mathcal{P}_{\text {rej }} \\
& =\frac{1-q_{\text {acc }} \mathcal{P}_{\text {acc }}}{1-\mathcal{P}_{\text {acc }}} \mathcal{P}_{\text {rej }} \\
& =\frac{1-\tilde{\mathrm{K}} / \hat{\mathrm{K}}}{1-\mathrm{K} / \hat{\mathrm{K}}} \mathcal{P}_{\text {rej }} \\
& =\frac{\hat{\mathrm{K}}-\tilde{\mathrm{K}}}{\hat{\mathrm{K}}-\mathrm{K}} \mathcal{P}_{\text {rej }}=q_{\text {rej }} \mathcal{P}_{\text {rej }} .
\end{aligned}
$$

\subsubsection{Cut-off issues and numerical instabilities}

The backwards-evolution of initial-state parton branchings $a \rightarrow b$ is guided by PDF ratios $f_{a}\left(x / z, \mu_{F}^{2}\right) / f_{b}\left(x, \mu_{F}^{2}\right)$. It is easy to see that this complicates choosing an over-estimator $\hat{\mathrm{K}}$, with $\hat{\mathrm{K}} \geq \mathrm{K}$ everywhere. A pragmatic solution is to find one which fulfills the relation almost everywhere, and otherwise cap the emission probability at 1 . The ratio can even get negative for PDF sets that are not positive-definite. This translates to negative probabilities in the SVA. Again, one can artificially enforce a minimum probability of zero to prevent this.

These choices are employed in CSSHOwER. For the reweighting, such cut-offs could be an issue, if they occur too often. The problem is that the affected phase-space regions will not perfectly overlap for different parameter/scale choices. For example, consider a trial emission, where using the nominal PDF leads to a negative emission probability, which is then set to zero. The trial emission is therefore rejected. However, the target PDF of 

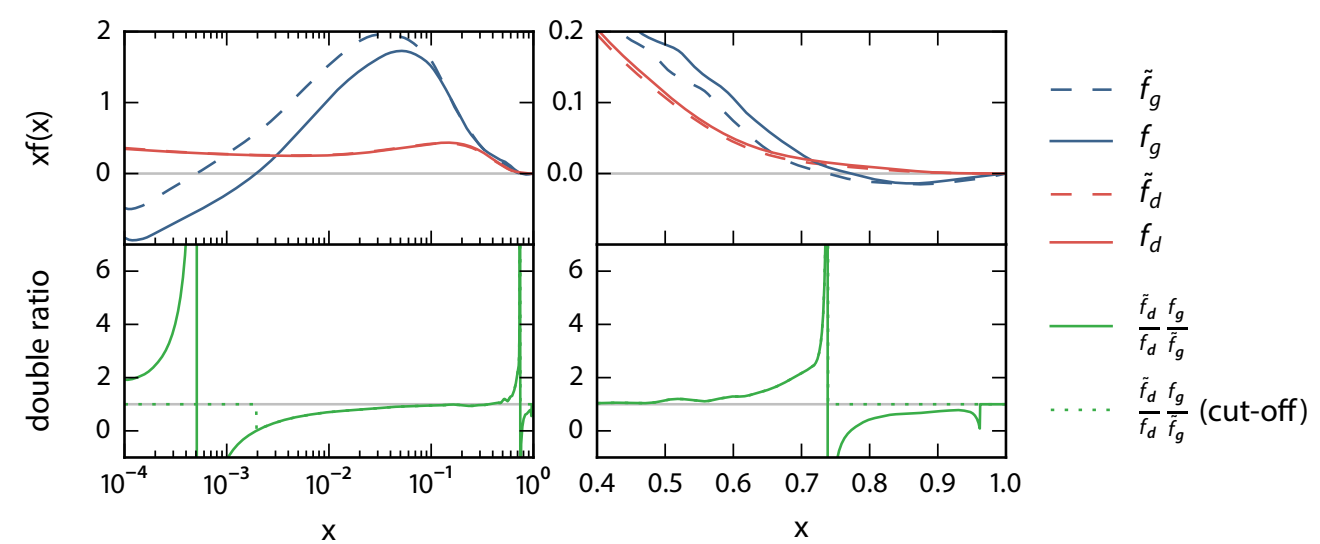

Figure 3.2.: Illustration of two problems related to the reweighting of initial-state partonshower emissions, for the example of a low-scale $g \rightarrow d \bar{d}$ splitting. The PDF double ratios as depicted in the lower panels feature divergences and their constituents-individually shown in the upper panels-drop below zero in different $x$ ranges for different PDF variants $f$ and $\tilde{f}$. These are chosen to be the NNPDF 3.0 sets fitted to $a_{s}\left(m_{Z}^{2}\right)=0.118$ and $a_{s}\left(m_{Z}^{2}\right)=0.115$, respectively.

the reweighting might lead to a finite PDF ratio, and hence to a finite emission probability. However, the first line of Eq. (3.25) will give us a reweighting factor $q_{\mathrm{rej}}=1$, which is clearly not correct. Note that using the last line in Eq. (3.25) would give us a different answer, which even more highlights the pathology of reweighting such a trial emission. A related cut-off issue is caused by the minimum $x$ and $\mu_{F}^{2}$ values of the involved PDF variations, which potentially differ if the variations are from different PDF sets.

A separate issue related to PDF ratios is the numerical stability of the reweighting. When calculating weight factors for the reweighting, ratios of these ratios will occur. Although most of these double ratios are expected to be around unity, individual ones can become very large, if the PDFs in the denominators approach zero at different $\left(x, \mu_{F}^{2}\right)$ values.

We illustrate the negativity and the large reweighting factor issue in Fig. 3.2 for a reweighting of $\alpha_{S}\left(m_{\mathrm{Z}}^{2}\right)=0.118$ down to o.115. Remember that this entails a change in the associate PDFs, too. We plot the PDF double ratio and its constituent PDFs over $x$ for a $g \rightarrow$ da splitting, i.e. an initial-state down quark is backwards-evolved to a gluon. The scale is set to $\mu_{F}^{2} \gtrsim 1 \mathrm{GeV}^{2}$, and for the ratio the fraction of partonic momentum carried over is set to unity, $z=1$. As such, we are considering a rather extreme trial emission, but it proves our point, especially because we use NNPDF 3.0 , which is not a positive-definite PDF set.

In Fig. 3.2, one can see that for the gluon PDF, $f_{\mathrm{g}}$, the crossing of zero occurs at smaller values of $x$ for $\alpha_{S}\left(m_{\mathrm{Z}}^{2}\right)=0.118$, both for the crossing at small and at high $x$. The opposite is the case for the down-quark PDF, $f_{\mathrm{d}}$, which drops below zero for a smaller $x$ value for $\alpha_{S}\left(m_{\mathrm{Z}}^{2}\right)=0.115$, and only once, at high $x$. This leads to two $x$ regions at which one PDF ratio 
is positive, and the other is not. In our implementation, we omit any reweighting factor involving negative PDF values, i.e. we fall back to $q_{\mathrm{acc}}=1$ or $q_{\mathrm{rej}}=1$, which is indicated by the dashed line in the figure. This is a pragmatic solution, but is expected to work for most observables. In the end, this choice resembles the one taken in CSSHOWER, and should therefore lie within its inherent algorithmic uncertainty. The minimum $\mu_{F}^{2}$ cut-off issue is treated the same way. The number of such occurrences is monitored during event generation for inspection.

We also see divergences of the PDF double ratio as expected. This is not restricted to PDF that can drop below zero. Also positive-definite PDFs can approach or become equal to zero. Single trial emissions could then lead to very large event weights, although their impact on the actual observable might be negligible. Although still being valid in the limit of asymptotic statistics, the reweighting might feature very large statistical errors for a finite sample, even if the nominal distribution is smooth.

The same is true, if the over-estimate function $\hat{\mathrm{K}}$ resembles the nominal kernel $\mathrm{K}$ too closely, because the denominator of the rejection weight factor $q_{\text {rej }}$ is $(\hat{\mathrm{K}}-\mathrm{K})$, see Eq. (3.25). One approach to that problem is to give some leeway by introducing a prefactor $k_{\hat{\mathrm{K}}}$ to $\hat{K}$, cf. $[139,140,151]$. This renders the SVA less efficient, but is shown to reduce statistical fluctuations in the reweighting.

However, in our implementation we address both issues-large PDF double ratios and "too perfect" over-estimators-by introducing a cut-off. If a trial emissions would result in a reweighting factor $\mathcal{Q}$ which deviates more than ten from unity, it is not reweighted at all. The effect of this prescription is illustrated in Fig. 3.3, where we have generated two sets of uncertainty bands using the internal reweighting for the W-boson transverse momentum in $\mathrm{W}$ production at a $13 \mathrm{TeV}$ pp collider. The matrix element and all parton-shower emissions are reweighted in the nominal LO plus parton-shower event generation, with and without the $\mathcal{Q}$ cut-off. We observe that the spikes associated to very large reweighting factors disappear after enforcing the cut-off. In this case, the spikes were restricted to the $\mathrm{CT}_{14}$ PDF uncertainty variation. Otherwise, the uncertainty bands do not change. This proves that we do not change the reweighting for the bulk of the trial emissions.

Although the cut-off approach proved to cure the numerical issues for the $\mathrm{W}$ transverse momentum, we will see for dijet azimuthal decorrelations in Section 3.12, that a $\hat{K}$ prefactor $k_{\hat{\mathrm{K}}}$ might be necessary to provide additional numerical stability for other observables/processes.

However, even if very large reweighting factors are omitted, we can still ask if the reweighting will broaden the nominal weight distribution too strongly to be practical. The answer depends on the hard process and on the observable, and therefore we will need more experience to give it. Still, very encouraging examples are presented in the following sections. For a first exploration, we look at the weight distributions for trial emissions of a simplified parton shower evaluated using the reweighted SVA implementation. This parton shower 


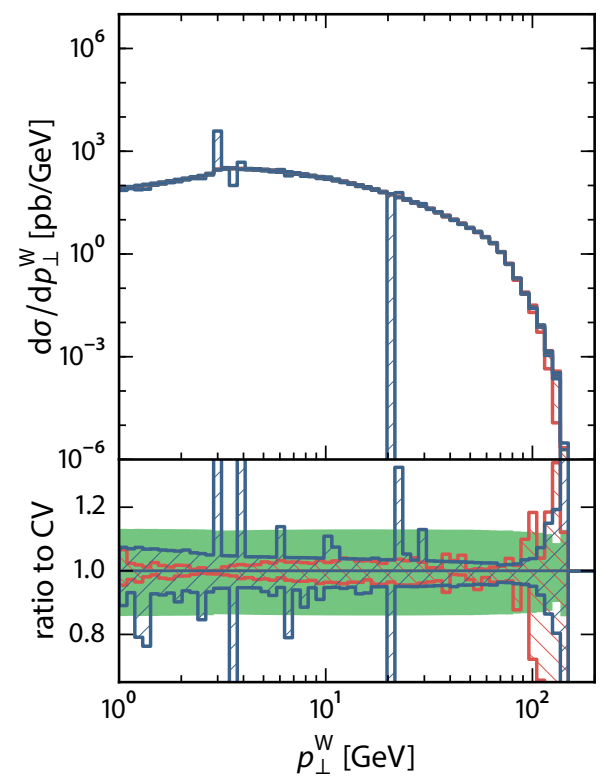

(a) include all reweighting factors

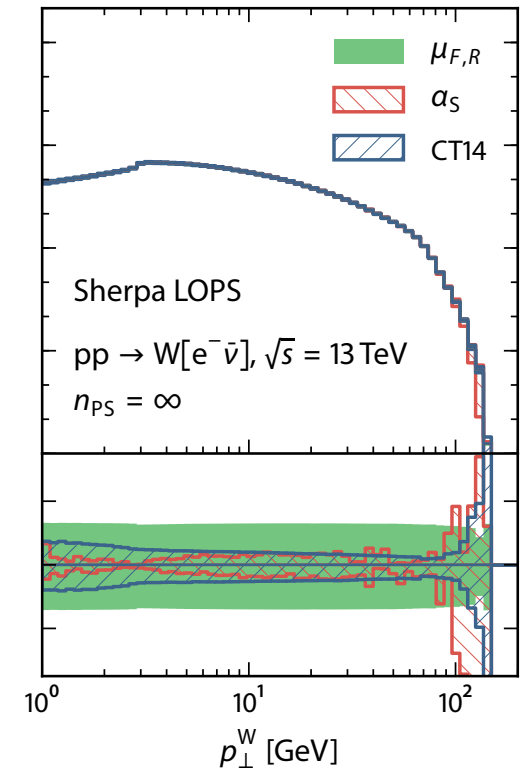

(b) veto large reweighting factor

Figure 3.3.: Uncertainty bands for the transverse momentum of the $\mathrm{W}$ in LO plus partonshower $W$ production for independent variations of $\mu_{F}$ and $\mu_{R}$ (green), $a_{S}$ (red) and the PDF (blue). The uncertainty bands are generated by a reweighting of the central event generation run. On the left-hand side, all reweighting factors $\mathcal{Q}$ were applied to the event weights, whereas on the right-hand side, reweighting factors $\mathcal{Q}$ with $|\mathcal{Q}-1|>10$ have been discarded.

uses an emission kernel as defined in Eq. (3.10) given by

$$
J(t, z, \phi) \mathrm{K}_{i j}(t, z, \phi)=\frac{1}{t^{2}} P_{i j}(z) \frac{f_{i}\left(x / z, t^{2}\right)}{f_{j}\left(x, t^{2}\right)} .
$$

This means that we are simulating a backwards initial-state shower, ordered in $t$, with splitting functions $P_{i j}$. The starting conditions are $x=0.2$ and $t_{\max }=1000 \mathrm{GeV}^{2}$, and a cut-off value of $t_{\mathrm{IR}}=1 \mathrm{GeV}^{2}$ is set. To constrain the shower to resolvable (and hence finite) emissions, the $z$ integral limits are set to $t_{\max }^{2} / t^{2}$ and $1-t_{\max }^{2} / t^{2}$.

We generate two independent samples, one for $\mathrm{u} \rightarrow \mathrm{ug}$ emissions, and one for $\mathrm{g} \rightarrow \mathrm{u} \overline{\mathrm{u}}$ splittings, using the NNPDF 3.0 set. Generating splitting chains for only one channel is unphysical (especially in the gluon splitting case, which can not succeed itself), but makes it easy to study their individual reweighting factor distributions. The channels and their choices for $P_{i j}$ and $\hat{\mathrm{K}}$ are listed in Table 3.1. The $\mathrm{u} \rightarrow \mathrm{ug}$ sample consists of 250 statistically independent runs, and the $g \rightarrow$ uu sample of 1000 runs. We record the $t$ values for each accepted trial emission and plot the $t$ histogram ("splitting frequency"). We also do a reweighting for variations over all 100 NNPDF 3.0 set replicas, and plot the corresponding 
Table 3.1.: The two channels of the simplified parton shower with their splitting functions $P_{i j}$ and their over-estimate functions $\hat{\mathrm{K}}$.

\begin{tabular}{lll}
\hline branching & $P_{i j}$ & $\hat{\mathrm{K}}$ \\
\hline $\mathrm{u} \rightarrow \mathrm{ug}$ & $P_{\mathrm{qq}}=\frac{1+z^{2}}{1-z}$ & $\frac{1}{t^{2}} \frac{4}{1-z}$ \\
$\mathrm{~g} \rightarrow \mathrm{uu} \overline{\mathrm{u}}$ & $P_{\mathrm{gq}}=\frac{1+(1-z)^{2}}{z}$ & $\frac{1}{t^{2}} \frac{4}{z}$ \\
\hline
\end{tabular}

PDF uncertainty. Most importantly, we also plot the product of reweighting factors for the PDF reweighting from the central NNPDF 3.0 member to its 1ooth replica, i.e. the $\mathcal{Q}$ as defined below Eq. (3.17).

The results are shown in Fig. 3.4. Looking at the left-hand panels, i.e. the normalised distribution of $t$-values, at which splittings occur, we find that the $g \rightarrow u \bar{u}$ spikes more strongly at low values of $t$, and falls down more quickly towards higher $t$ values. Also, the PDF uncertainty is much larger in the $g \rightarrow u \bar{u}$ case (note the different $y$ scales in the ratio plots), which is a result of the larger gluon PDF uncertainty.

In the right-hand panels, the reweighting factors $\mathcal{Q}$ are presented for the reweighting to the 10oth NNPDF 3.0 replica. For the $\mathrm{u} \rightarrow$ ug emissions, the $\mathcal{Q}$ are distributed in a narrow band around 1.0 with a width of $\mathcal{O}(1 \%)$. This situation is very different for the $\mathrm{g} \rightarrow$ uu splittings. Here, the bulk of the weights is located around $\sim 0.9$, with an extended tail towards larger values, which becomes more extended for smaller values of $t$. This clearly indicates that the reweighting would suppress events with large- $t$ emissions and enhance those with small- $t$ emissions. Far outliers also foreshadow that the weight distribution of full Monte Carlo events will be broadened by the reweighting. We conclude that this must be monitored for hadronic collisions that feature initial-state radiation, to ensure that the statistical accuracy of reweighted results is not diminished significantly.

\subsection{LO matrix elements and parton showers}

We leave now the pure parton-shower test case and consider again more practical applications, i.e. full event generations. If parton-showering is added to a LO calculation, the value of the observable is not evaluated at $\Phi_{B}$ any longer, but at $\operatorname{PS}\left(\Phi_{B}\right)$, which denotes the phase-space point after the showering. Applying this modification to Eq. (3.1) yields

$$
O^{\mathrm{LOPS}}=\int \mathrm{d} \Phi_{B} \mathrm{~B}\left(\Phi_{B}\right) \operatorname{PS}\left(O, \Phi_{B}\right)=\lim _{N \rightarrow \infty} \frac{1}{N_{\text {trials }}} \sum_{i=1}^{N} \mathrm{~B}\left(\Phi_{B, i}\right) \operatorname{PS}\left(O, \Phi_{B, i}\right) .
$$



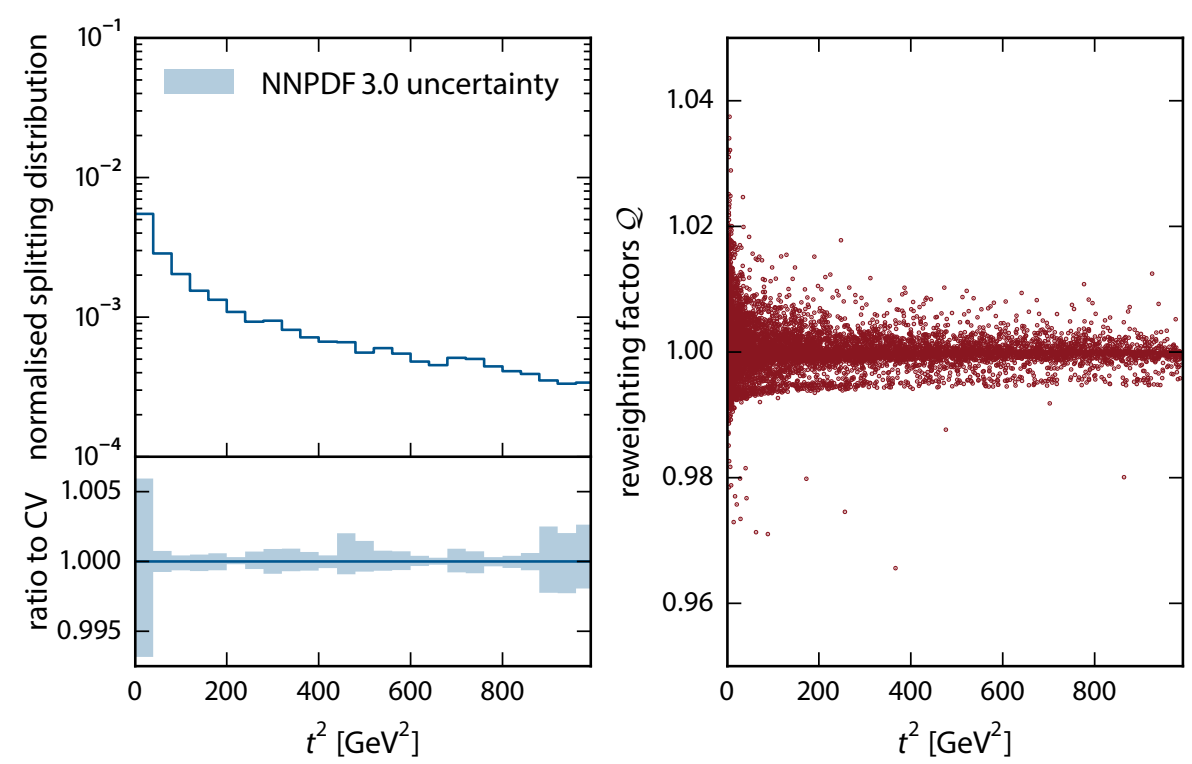

(a) $u \rightarrow u g$
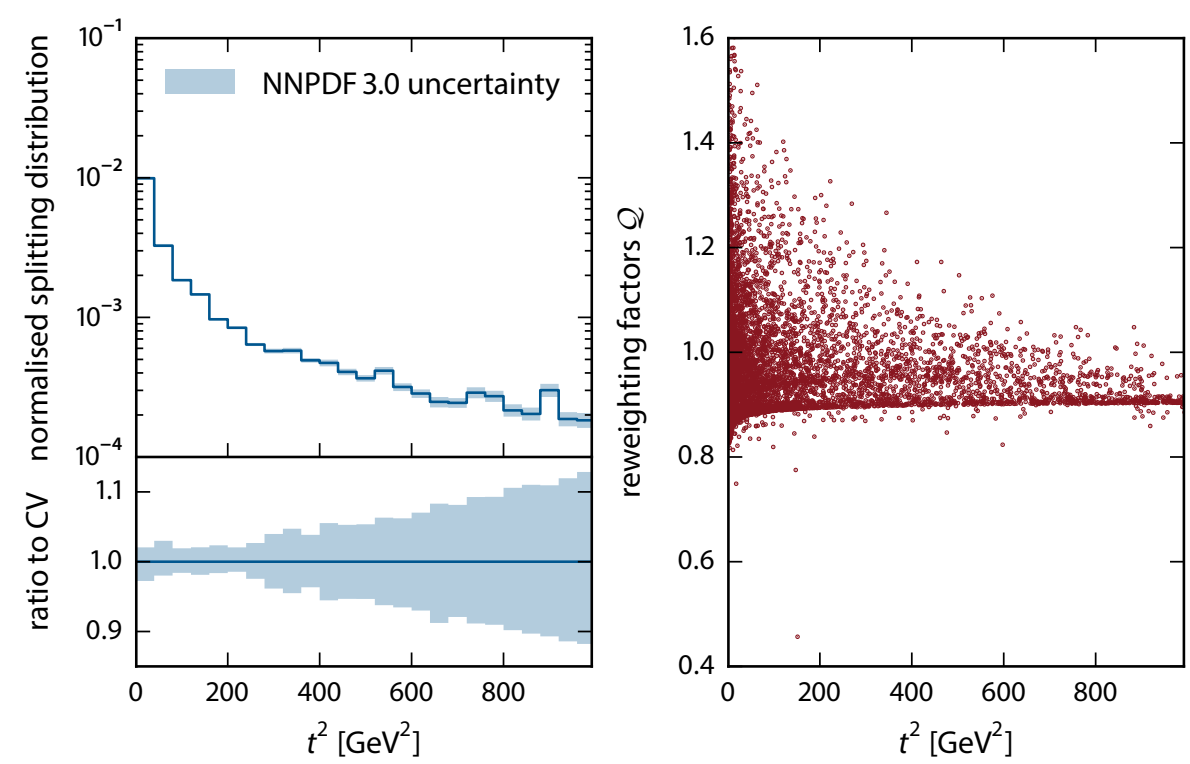

(b) $g \rightarrow$ uū

Figure 3.4.: The distribution of the evolution variable $t^{2}$, at which splittings occur, for the $\mathrm{u} \rightarrow \mathrm{ug}$ and the $\mathrm{g} \rightarrow \mathrm{uu}$ channels. The distribution is varied over all replicas using the reweighted SVA. For the 100th replica, the reweighting factors $\mathcal{Q}$ for each splitting are shown in the right-hand panels. 
Therefore the reweighting for B does not need to be altered, but the parton-shower emissions depend on the PDF, the strong coupling, their respective scale prefactors $k_{f}$ and $k_{\alpha_{S}}$ (detailed below) and its starting scale $\mu_{\mathrm{Q}}$ :

$$
\operatorname{PS}\left(O, \Phi_{B}\right) \equiv \operatorname{PS}\left(O, \Phi_{B} ; \mu_{Q}^{2}\right) \equiv \operatorname{PS}\left(O, \Phi_{B} ; k_{\alpha_{S}}, k_{f} ; \alpha_{S}, f ; \mu_{Q}^{2}\right) .
$$

In order to reweight the parton-shower emissions, we first need to identify its exact dependence structure. Schematically, it acts on the phase-space element in the following way

$$
\operatorname{PS}\left(O, \Phi_{n} ; t^{\prime}\right)=\Delta_{n}\left(t_{\mathrm{IR}}, t^{\prime}\right) O\left(\Phi_{n}\right)+\int_{t_{\mathrm{IR}}}^{t^{\prime}} \mathrm{d} \Phi_{1} \mathrm{~K}_{n}\left(\Phi_{1}\right) \Delta_{n}\left(t, t^{\prime}\right) \operatorname{PS}\left(O, \Phi_{n+1} ; t\right)
$$

where the Sudakov form factor of the $n$-parton state, $\Delta_{n}$, and its emission kernel $\mathrm{K}_{n}$ have been introduced. While the first term describes the no-emission probability between the starting scale $t^{\prime}$ and the infrared cut-off $t_{\mathrm{IR}}$ and therefore does not change the phase-space element, the second term describes the emissions of a parton at scale $t$ in the configuration $\mathrm{d} \Phi_{1}=\mathrm{d} t \mathrm{~d} z \mathrm{~d} \phi J(t, z)$ (the integration boundaries are to be understood in this decomposition), leading to a configuration $\mathrm{d} \Phi_{n+1}=\mathrm{d} \Phi_{n} \cdot \mathrm{d} \Phi_{1}$. The Jacobian $J$ is not relevant to the discussion here and is subsequently absorbed in the emission kernel $\mathrm{K}_{n}$. As the emissions are ordered in $t$, the Sudakov form factor in the second term ensures that the current emission is the hardest after starting the evolution at $t^{\prime}$. Additional branchings may occur at smaller $t$ and are not resolved at this stage-they are described by the parton shower acting on the newly produced state $\Phi_{n+1}$ with the new starting scale $t$. In Eq. (3.29) the dependences on $\alpha_{S}$, the PDFs, and their respective scale prefactors $k_{\alpha_{S}}$ and $k_{f}$ have been omitted for brevity. They directly carry over to the emission kernel and the Sudakov form factor, according to

$$
\Delta_{n}\left(t_{2}, t_{1} ; k_{\alpha_{S}}, k_{f} ; \alpha_{S}, f\right)=\exp \left(-\int_{t_{2}}^{t_{1}} \mathrm{~d} \Phi_{1} \mathrm{~K}_{n}\left(\Phi_{1} ; k_{\alpha_{S}}, k_{f} ; \alpha_{S}, f\right)\right) .
$$

When considering parton-shower emissions off NLO QCD matrix elements, special emphasis has to be given to the first emission as described in Section 3.5 below.

The default parton shower of SHERPA, dubbed CSSHOwER, is based on Catani-Seymour dipole factorisation [76], as discussed in Section 2.2.2. Each splitting of an emitter parton into two daughters is witnessed by a spectator parton, which takes the recoil, and ensures that on-shell states are transferred into on-shell states and energy-momentum conservation is respected simultaneously. The emitter and spectator partons reside either in the initialstate (I) or final-state (F), such that four dipole types need to be distinguished: II, IF, FI and FF. In this notation, the first letter refers to emitter, and the second to the spectator parton. The no-emission probabilities are given by the four corresponding Sudakov form factors,

$$
\Delta_{n}\left(t_{2}, t_{1} ; k_{\alpha_{S}}, k_{f} ; \alpha_{S}, f\right)=\prod_{\text {type } \in\{\mathrm{FF}, \mathrm{FI}, \mathrm{IF}, \mathrm{II}\}} \Delta_{n}^{\mathrm{type}}\left(t_{2}, t_{1} ; k_{\alpha_{S}}, k_{f} ; \alpha_{S}, f\right) .
$$


Table 3.2.: Definition of the evolution and splitting variables for each dipole type. The fifth column lists the splitting process as seen from the Born process, $c$ and $c^{\prime}$ refer to the flavour of the initial state before and after the splitting process, respectively. The variables $y_{i j, k}, \tilde{z}_{i}$, $x_{i j, a}, x_{j k, a}, x_{j, a b}, u_{j}$ and $v_{j}$ are defined in $[21,76]$.

\begin{tabular}{|c|c|c|c|c|c|c|}
\hline Diagram & Type & $z$ & $y$ & $x$ & $(i j, k) \rightarrow(i, j, k)$ & $c, c^{\prime}$ \\
\hline & $\mathrm{FF}$ & $\tilde{z}_{i}$ & $y_{i j, k}$ & 1 & $(i j, k) \rightarrow(i, j, k)$ & $a, a$ \\
\hline & FI & $\tilde{z}_{i}$ & $\frac{1-x_{i j, a}}{x_{i j, a}}$ & $x_{i j, a}$ & $(i j, a) \rightarrow(i, j, a)$ & $a, a$ \\
\hline & IF & $x_{j k, a}$ & $\frac{u_{j}}{x_{j k, a}}$ & $x_{j k, a}$ & $(a j, k) \rightarrow(a, j, k)$ & $a j, a$ \\
\hline & II & $x_{j, a b}$ & $\frac{\tilde{v}_{j}}{x_{j, a b}}$ & $x_{j, a b}$ & $(a j, b) \rightarrow(a, j, b)$ & $a j, a$ \\
\hline
\end{tabular}

They share the common form

$$
\begin{aligned}
& \Delta_{n}^{\text {type }}\left(t_{2}, t_{1} ; k_{\alpha_{S}}, k_{f} ; \alpha_{S}, f\right) \\
& \quad=\exp \left(-\sum_{i j} \sum_{k} \int_{t_{2}}^{t_{1}} \mathrm{~d} t \int_{z_{-}}^{z_{+}} \mathrm{d} z \alpha_{S}\left(k_{\alpha_{S}} t\right) \mathrm{K}_{i j, k}^{\prime}(t, z) \frac{f_{c^{\prime}}\left(\frac{\eta_{c}}{x}, k_{f} t\right)}{f_{c}\left(\eta_{c}, k_{f} t\right)}\right),
\end{aligned}
$$

wherein the kinematics of the splitting are given by the default choice for $t=Q^{2} y z(1-z)$ in the massless case while the $\mathrm{K}_{i j, k}^{\prime}(t, z)$ denote the coupling and PDF stripped emission kernels incorporating the remaining pieces of the $\mathrm{K}_{i j, k}$ and the Jacobian $J$ of the phase-space parametrisation. The precise definitions of the variables for each dipole type are given in Table 3.2. It directly follows that for FF-type dipole emissions the ratio of PDFs is simply unity. Equation (3.32) further details the dependence on the $\alpha_{S}$ and PDF scale factors $k_{\alpha_{S}}$ and $k_{f}$. These multiplicative factors as well as their variations are assumed to be of order one, such that they do not induce spurious large logarithms. The generalisation to the massive case is straightforward and only involves generalised definitions of $t, x, y$ and $z$, cf. [21]. 
We employ now the reweighted SVA stated in Section 3.3.2 to account for variations of the strong-coupling parameter and the PDFs in the shower evolution of LO and NLO QCD matrix elements. The emission kernels $\mathrm{K}$ depend linearly on $\alpha_{S}$ and on a ratio of PDFs $f_{c^{\prime}}\left(\eta_{c} / x, k_{f} t\right) / f_{c}\left(\eta_{c} / x, k_{f} t\right)$. A change of PDFs $f \rightarrow \tilde{f}$, the strong coupling $\alpha_{S} \rightarrow \tilde{\alpha}_{S}$ and the scale prefactors entering both, i.e. $k_{\alpha_{S}} \rightarrow \tilde{k}_{\alpha_{S}}$ and $k_{f} \rightarrow \tilde{k}_{f}$, gives a reweighting factor for each accepted trial emission ${ }^{5}$ :

$$
\mathcal{P}_{\mathrm{acc}} \rightarrow q_{\mathrm{acc}} \mathcal{P}_{\mathrm{acc}}, \quad q_{\mathrm{acc}} \equiv \frac{\tilde{\alpha}_{S}\left(\tilde{k}_{\alpha_{S}} t\right)}{\alpha_{S}\left(k_{\alpha_{S}} t\right)} \frac{\tilde{f}_{c^{\prime}}\left(\frac{\eta_{c}}{x}, \tilde{k}_{f} t\right)}{f_{c^{\prime}}\left(\frac{\eta_{c}}{x}, k_{f} t\right)} \frac{f_{c}\left(\eta_{c}, k_{f} t\right)}{\tilde{f}_{c}\left(\eta_{c}, \tilde{k}_{f} t\right)},
$$

where the scale dependence and the definition of $\eta_{c}$ and $x$ can be read off the Sudakov form factors given in Eq. (3.32) and Table 3.2. In case of FF dipoles, Eq. (3.33) simplifies significantly, as the ratios of PDF factors reduce to unity. As explained in Section 3.3.2, each $q_{\text {acc }}$ must be multiplied to the overall event weight to incorporate the new choice of $\alpha_{S}$, PDFs and the scales they are evaluated at. On the other hand, each rejected trial emission gives a reweighting factor to

$$
\mathcal{P}_{\text {rej }}=1-\mathcal{P}_{\text {acc }} \rightarrow 1-q_{\text {acc }} \mathcal{P}_{\text {acc }}=\left[1+\left(1-q_{\text {acc }}\right) \frac{\mathcal{P}_{\text {acc }}}{1-\mathcal{P}_{\text {acc }}}\right] \mathcal{P}_{\text {rej }} \equiv q_{\text {rej }} \mathcal{P}_{\text {rej }}
$$

Consequently, for each rejected emission the event weight receives a corrective weight of $q_{\text {rej. }}$

\subsection{NLO matrix elements and parton showers}

To match NLO QCD parton-level calculations with subsequent parton-shower evolution, SHERPA employs a variant of the original MC@NLO algorithm, referred to as S-MC@NLO, as has been discussed in Section 2.2.3. Schematically, such a S-MC@NLO calculation has

\footnotetext{
${ }^{5}$ Although the emission scales can not be reweighted themselves using the presented method, the input scales of the strong coupling and the PDFs can be changed, as indicated in the text. We focus on constant prefactors here, but the functional form can also be changed, although the overall functional form of $k_{\alpha_{S}} t$ should be restricted to the $\mathrm{CMW}$-like rescaling [156].
} 
the following structure:

$$
\begin{aligned}
&\langle O\rangle^{\mathrm{NLOPS}}=\int \Phi_{B} {\left[\mathrm{~B}\left(\Phi_{B}\right)+\mathrm{VI}\left(\Phi_{B}\right)+\int \mathrm{d} x_{a / b}^{\prime} \mathrm{KP}\left(\Phi_{B}, x_{a / b}^{\prime}\right)\right.} \\
&\left.+\sum_{j} \int \mathrm{d} \Phi_{1}^{j}\left(\mathrm{D}_{A, j}-\mathrm{D}_{S, j}\right)\left(\Phi_{B} \cdot \Phi_{1}^{j}\right)\right] \operatorname{PS}_{\mathrm{NLOPS}}\left(O, \Phi_{B}\right) \\
&+\int \Phi_{R}\left[\mathrm{R}\left(\Phi_{R}\right)-\sum_{j} \mathrm{D}_{A, j}\left(\Phi_{B}^{j} \cdot \Phi_{1}^{j}\right)\right] \operatorname{PS}\left(O, \Phi_{R}\right) \\
&=\int \mathrm{d} \Phi_{B} \overline{\mathrm{B}}\left(\Phi_{B}\right) \operatorname{PS}_{\mathrm{NLOPS}}\left(O, \Phi_{B}\right)+\int \mathrm{d} \Phi_{R} \mathrm{H}_{A}\left(\Phi_{R}\right) \operatorname{PS}\left(O, \Phi_{R}\right) .
\end{aligned}
$$

Here the real-emission contribution R of the NLO calculation has effectively been split into an infrared-singular (soft) and an infrared-regular (hard) part, the resummation kernel $\mathrm{D}_{A}$ and the finite hard remainder $\mathrm{H}_{A}$, respectively, such that $\mathrm{R}=\mathrm{D}_{A}+\mathrm{H}_{A}[87,157]$. The $\overline{\mathrm{B}}$-function has the following explicit parameter dependences,

$$
\begin{aligned}
\overline{\mathrm{B}}\left(\Phi_{B}\right) \equiv & \overline{\mathrm{B}}\left(\Phi_{B} ; \alpha_{S}, f ; \mu_{R}, \mu_{F}\right) \\
=\mathrm{B} & \left(\Phi_{B} ; \alpha_{S}, f ; \mu_{R}, \mu_{F}\right)+\operatorname{VI}\left(\Phi_{B} ; \alpha_{S}, f ; \mu_{R}, \mu_{F}\right) \\
& +\int \mathrm{d} x_{a / b}^{\prime} \mathrm{KP}\left(\Phi_{B}, x_{a / b}^{\prime} ; \alpha_{S}, f ; \mu_{R}, \mu_{F}\right) \\
& +\sum_{j} \int \mathrm{d} \Phi_{1}^{j}\left(\mathrm{D}_{A, j}-\mathrm{D}_{S, j}\right)\left(\Phi_{B} \cdot \Phi_{1}^{j} ; \alpha_{S}, f ; \mu_{R}, \mu_{F}\right) .
\end{aligned}
$$

From the perspective of parameter reweighting, the resummation kernel $\mathrm{D}_{A}$ contribution behaves the same way as the subtraction term $\mathrm{D}_{S}$. In fact, in our reweighting implementation the $\left(D_{A}-D_{S}\right)$ contribution is treated as a single term, as indicated in the last line of Eq. (3.36). Their PDFs are evaluated at the partonic momentum fractions $x_{a / b, j}$ and external flavours $a_{j}$ and $b_{j}$ of their $\Phi_{B} \cdot \Phi_{1}^{j}$ phase-space configuration rather than those of $\Phi_{B}$. The other parts of the $\overline{\mathrm{B}}$-function can then be reweighted as described in Section 3.1.2, leading to

$$
\begin{aligned}
& \overline{\mathrm{B}}\left(\Phi_{B} ; \tilde{\alpha}_{S}, \tilde{f}_{;} \tilde{\mu}_{R}, \tilde{\mu}_{F}\right) \\
& =\tilde{\alpha}_{S}^{n}\left(\tilde{\mu}_{R}^{2}\right) \tilde{f}_{a}\left(x_{a}, \tilde{\mu}_{F}^{2}\right) \tilde{f}_{b}\left(x_{b}, \tilde{\mu}_{F}^{2}\right) \\
& \quad \times\left[\mathrm{B}^{\prime}\left(\Phi_{B}\right)+\tilde{\alpha}_{S}\left(\tilde{\mu}_{R}^{2}\right)\left(\mathrm{VI}^{\prime}\left(\Phi_{B}\right)+c_{R}^{\prime(0)} l_{R}+\frac{1}{2} c_{R}^{\prime(1)} l_{R}^{2}\right)\right. \\
& \left.\quad+\tilde{\alpha}_{S}\left(\tilde{\mu}_{R}^{2}\right) \int \mathrm{d} x_{a / b}^{\prime} \mathrm{KP}^{\prime}\left(\Phi_{B}, x_{a / b}^{\prime} ; \tilde{f}_{j} \tilde{\mu}_{F}\right)\right] \\
& \quad+\sum_{j} \int \mathrm{d} \Phi_{1}^{j} \tilde{f}_{a_{j}}\left(x_{a_{j}}, \tilde{\mu}_{F}^{2}\right) \tilde{f}_{b_{j}}\left(x_{b_{j}}, \tilde{\mu}_{F}^{2}\right) \tilde{\alpha}_{S}^{n+1}\left(\tilde{\mu}_{R}^{2}\right)\left(\mathrm{D}_{A, j}-\mathrm{D}_{S, j}\right)\left(\Phi_{B} \cdot \Phi_{1}^{j}\right) .
\end{aligned}
$$


The $\mathrm{H}_{A}$-function transforms as

$$
\begin{aligned}
& \mathrm{H}_{A}\left(\Phi_{R} ; \tilde{\alpha}_{S}, \tilde{f} ; \tilde{\mu}_{R}, \tilde{\mu}_{F}\right) \\
& =\mathrm{R}\left(\Phi_{R} ; \tilde{\alpha}_{S}, \tilde{f} ; \tilde{\mu}_{R}, \tilde{\mu}_{F}\right)-\sum_{j} \mathrm{D}_{A, j}\left(\Phi_{B}^{j} \cdot \Phi_{1}^{j} ; \tilde{\alpha}_{S}, \tilde{f} ; \tilde{\mu}_{R, j}, \tilde{\mu}_{R, j}\right) \\
& =\tilde{\alpha}_{S}^{n+1}\left(\tilde{\mu}_{R}^{2}\right) \tilde{f}_{a_{j}}\left(x_{a_{j}}, \tilde{\mu}_{F}^{2}\right) \tilde{f}_{b_{j}}\left(x_{b_{j}}, \tilde{\mu}_{F}^{2}\right) \mathrm{R}^{\prime}\left(\Phi_{R}\right) \\
& \quad-\sum_{j} \tilde{\alpha}_{S}^{n+1}\left(\tilde{\mu}_{R, j}^{2}\right) \tilde{f}_{a_{j}}\left(x_{a_{j}}, \tilde{\mu}_{F, j}^{2}\right) \tilde{f}_{b_{j}}\left(x_{b_{j}}, \tilde{\mu}_{F, j}^{2}\right) \mathrm{D}_{A, j}^{\prime}\left(\Phi_{B}^{j} \cdot \Phi_{1}^{j}\right),
\end{aligned}
$$

wherein each subtraction term $\mathrm{D}_{A, j}$ has its own scales $\mu_{R, j}, \mu_{F, j}$ defined on its underlying Born configuration $\Phi_{B}^{j}$. Writing Eq. (3.35) as a Monte Carlo sum over events with B-like and R-like structure, which are conventionally called $\mathbb{S}$ and H events in MC@NLO calculations, and with $N=N_{\mathbb{S}}+N_{\mathbb{H}}$, we obtain:

$$
\begin{aligned}
\langle O\rangle^{\mathrm{NLOPS}}=\lim _{N \rightarrow \infty} \frac{1}{N_{\text {trials }}}\left\{\sum_{i=1}^{N_{\mathrm{S}}}\right. & \mathbb{S}\left(\Phi_{B, i}\right) \operatorname{PS}_{\mathrm{NLOPS}}\left(O, \Phi_{B, i}\right) \\
& \left.+\sum_{i=1}^{N_{\mathrm{H}}} \mathrm{H}\left(\Phi_{R, i}\right) \operatorname{PS}\left(O, \Phi_{R, i}\right)\right\} .
\end{aligned}
$$

Thus, under $\mu_{R} \rightarrow \tilde{\mu}_{R}, \mu_{F} \rightarrow \tilde{\mu}_{F}, \alpha_{S} \rightarrow \tilde{\alpha}_{S}$ and $f \rightarrow \tilde{f}$ both $\overline{\mathrm{B}}$ of the $\mathbb{S}$-events and $\mathrm{H}_{A}$ of the $\mathrm{H}$-events transform as composite objects in terms of their constituents, as defined above.

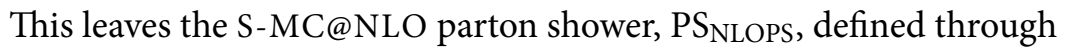

$$
\begin{aligned}
\operatorname{PS}_{\mathrm{NLOPS}}\left(O, \Phi_{B}\right) \equiv & \operatorname{PS}_{\mathrm{NLOPS}}\left(O, \Phi_{B} ; k_{\alpha_{S}}, k_{f} ; \alpha_{S}, f ; \mu_{Q}\right) \\
= & \bar{\Delta}_{n}\left(t_{\mathrm{IR}}, t^{\prime}\right) O\left(\Phi_{n}\right) \\
& \quad+\int_{t_{\mathrm{IR}}}^{t^{\prime}} \mathrm{d} \Phi_{1} \frac{\mathrm{D}_{A}\left(\Phi_{B} \cdot \Phi_{1}\right)}{\mathrm{B}\left(\Phi_{B}\right)} \bar{\Delta}_{n}\left(t, t^{\prime}\right) \operatorname{PS}\left(O, \Phi_{n+1} ; t\right) .
\end{aligned}
$$

It differs from the usual parton shower (PS) of Eq. (3.29) with respect to the splitting kernel for the first emission and the associated definition of the Sudakov form factor, cf. [87, 158]. However, for the purpose of reweighting, all trial emissions can be treated in the same way as in the standard parton shower, because the parameter dependences are identical.

\subsection{Closure tests for (N)LO matrix elements and parton showers}

In order to validate the reweighting of scale and parameter dependences in CSSHOWER and S-MC@NLO calculations within the SHERPA framework we perform closure tests between reweighting results and dedicated simulations.

Our implementation allows to constrain the maximum number of reweighted shower emissions per event. For a pure LO plus parton-shower run or H-like events in S-MC@NLO 
calculations, this amounts to setting $n_{\mathrm{PS}} \in\{0,1,2, \ldots, \infty\}$. When considering S-MC@NLO simulations in addition the parameter $n_{\text {NLOPS }} \in\{0,1\}$ can be used to disable the reweighting of the $\mathcal{O}\left(\alpha_{S}\right)$ emission for $\mathbb{S}$ events.

Of course the reweighting result will only coincide with a dedicated calculation if all emissions are reweighted, i.e. $n_{\mathrm{NLOPS}}=1$ and $n_{\mathrm{PS}}=\infty$. However, by subsequently enabling the reweighting of more and more emissions the relevance of their dependences for the determination of the full uncertainty can be studied. A finite value of $n_{\text {PS }}$ can also be useful in production, if the effect of reweighting higher-order emissions becomes negligible. The reduced amount of reweighting per event then allows for a faster event generation. An additional benefit would be that rare high-multiplicity shower histories do not spoil the statistical convergence of the reweighted result, even if their exact kinematics might be irrelevant for the studied observable.

\subsubsection{The final-state only case: Thrust and jet resolutions in $\mathrm{e}^{+} \mathrm{e}^{-} \rightarrow \mathrm{q} \overline{\mathrm{q}}$ events}

To validate LOPS reweighting, we consider two observables, which are complementary in their sensitivity to parton-shower emissions. At first, we consider the event-shape variable thrust $T$ [159] in hadronic events in $\mathrm{e}^{-} \mathrm{e}^{+}$collisions at $\sqrt{s}=91.2 \mathrm{GeV}$.

In this case, QCD emissions are restricted to the final state. Accordingly, there appear no PDF factors in the shower reweighting, cf. Eq. (3.33). Also there is no factorisation scale dependence in the matrix element for $\mathrm{e}^{+} \mathrm{e}^{-}$collision. Moreover, as we consider the leadingorder matrix element for $\mathrm{e}^{-} \mathrm{e}^{+} \rightarrow \mathrm{q} \overline{\mathrm{q}}$ only, the renormalisation scale is also absent in the hard-process component. Therefore, we can concentrate on the pure $\alpha_{S}$ uncertainty in the parton shower here. Leaving the perturbative order of its running invariant it is defined by its value at the input scale $m_{Z}$.

In Fig. 3.5, we compare $\alpha_{S}$ uncertainty bands generated by reweighting the nominal prediction with the one generated by dedicated predictions for each variation. As in Fig. 3.1, the uncertainty band is defined as the envelope over the distributions with different $\alpha_{S}\left(m_{\mathrm{Z}}^{2}\right)$ input values. The nominal value is taken as $\alpha_{S}\left(m_{Z}^{2}\right)=0.120$, and its up/down variations are 0.128 and 0.108 , respectively. Reweighting bands are presented for $n_{\mathrm{PS}}=1,4,8, \infty$. The $n_{\mathrm{PS}}=1$ band underestimates the uncertainty, especially for $T \leq 2 / 3$, where multiple hard emissions are required, and for $T \approx 1$, the region sensitive to multiple soft emissions. For $n_{\mathrm{PS}}=4$, the uncertainty is underestimated only for bins with $T \leq 2 / 3$, and less so than for the $n_{\mathrm{PS}}=1$ case. The difference between the two choices of $n_{\mathrm{PS}}=8$ and $\infty$ is merely statistical and both reproduce the dedicated result very accurately.

However, for low values of $T$, the statistical fluctuations of the reweighting results with higher $n_{\mathrm{PS}}$ grow larger, see the lower panel of Fig. 3.5, corresponding to a widening of the distribution of reweighting factors. Low values of the thrust observable correspond to the emission of several hard partons, which is less probable in the parton-shower approximation, and more appropriately modelled in multijet-merged calculations, cf. Section 3.7. In this phase-space region it is difficult for the reweighting to compensate the multitude of accepted soft emissions off these hard legs, that turn unstable for $\mathcal{P}_{\text {acc }} \rightarrow 1$, with rejected 

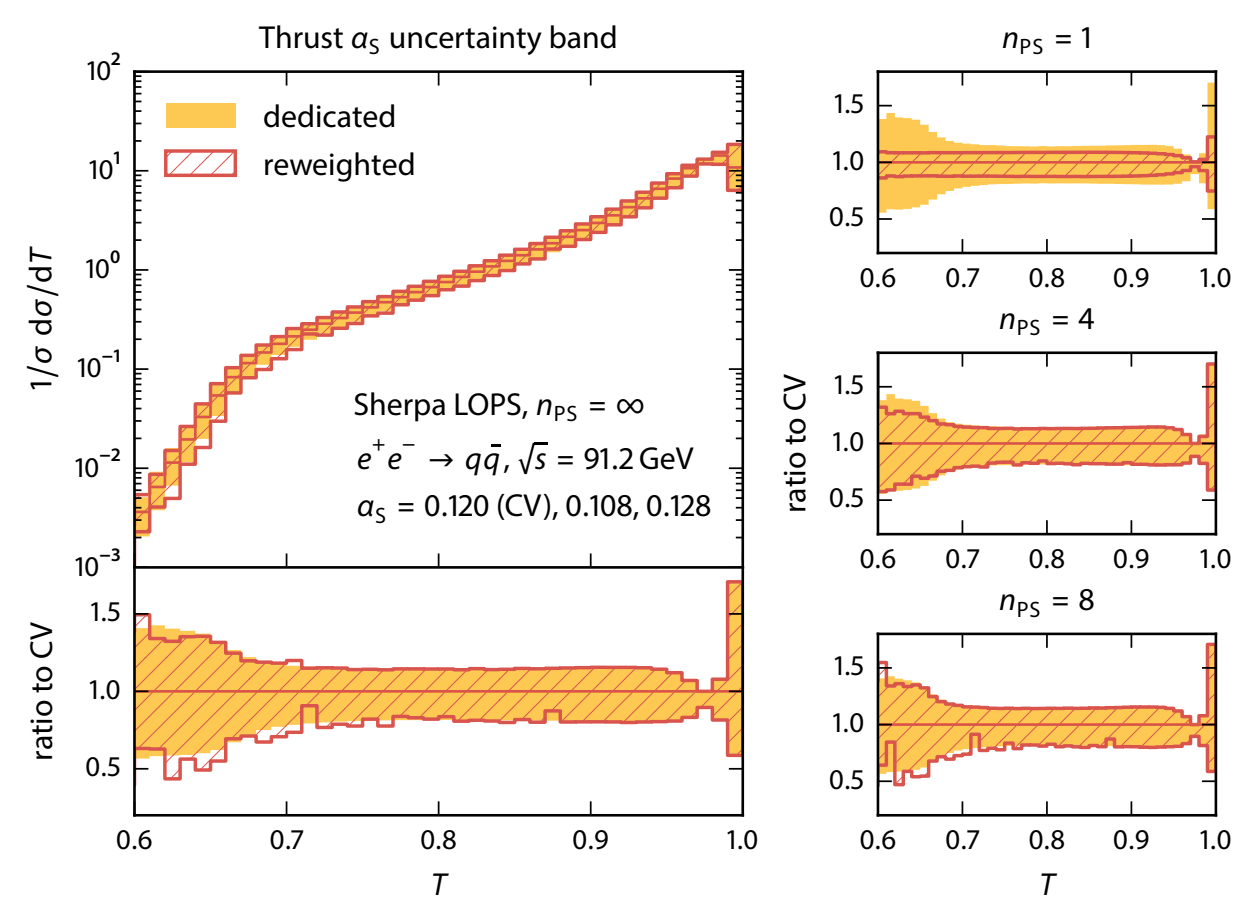

deviations from dedicated variations (statistical errors)
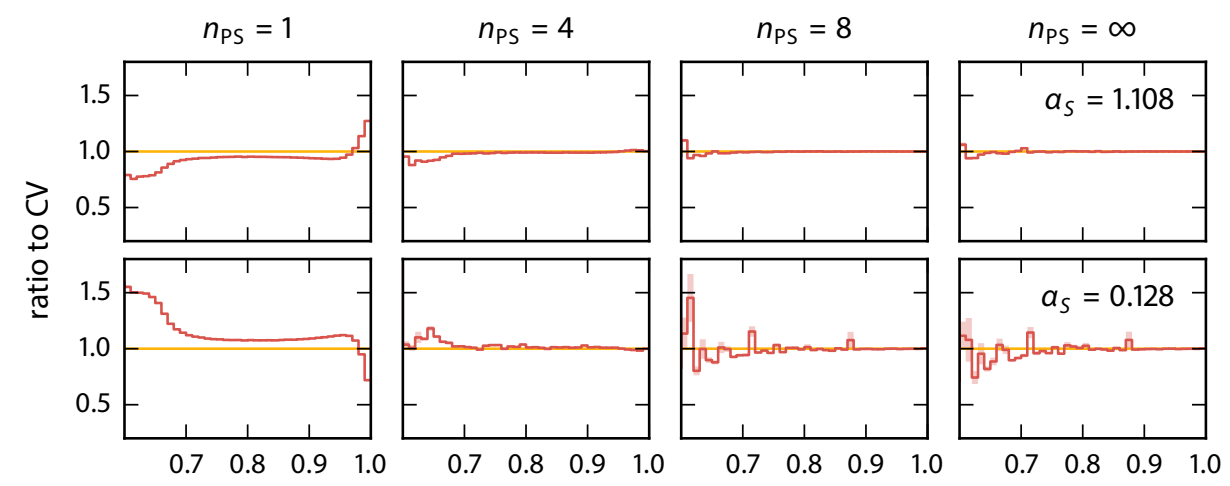

$T$

Figure 3.5.: Uncertainty band for the Thrust event shape in dijet production in $\mathrm{e}^{-} \mathrm{e}^{+}$annihilation for a variation of $a_{5}$. The upper left panel shows the nominal distribution and the ratio to the central value. The uncertainty band calculated using reweighting (including all emissions, i.e. $n_{\mathrm{PS}}=\infty$ ) is compared to the one obtained from dedicated calculations. The comparison is repeated in the three panels on the upper right for different maximum number of reweighted emission $n_{\text {PS. }}$. The lower panels show the deviations for each variation across the different values of $n_{\mathrm{PS}}$, including statistical errors. 
ones, cf. Eq. (3.34). As discussed at the end of Section 3.3, this issue might be addressed by introducing a prefactor to the over-estimator function $\hat{\mathrm{K}}$ in the reweighting run.

We supplement the validation of the reweighting with respect to the Thrust observable with another observable, the Durham jet resolution for a $(n+1) \rightarrow n$ clustering. The resolution parameter $y_{n, n+1}$ is defined as the value of the jet cut parameter $y_{\text {cut }}$ of the Durham jet algorithm [108], at which a $n$-jet configuration is clustered to a $(n+1)$-jet configuration. The distribution for $y_{n, n+1}$ is sensitive to the probability of hard-parton radiation from a $n$-jet configuration. So if we test the parton-shower reweighting, we are only sensitive up to the $(n+1)$ th emission, factorising out higher-multiplicity emissions. In this sense, the jet resolutions are more clean than the Thrust, where overlapping regions are sensitive to a varying number of emissions.

In Fig. 3.6, we provide cross-closure tests for a reweighting from $\alpha_{S}\left(m_{\mathrm{Z}}^{2}\right)=0.120$ to 0.128 , varying $n_{\mathrm{PS}}$. As expected, the reweighting succeeds when $n_{\mathrm{PS}} \geq n+1$. Even $n_{\mathrm{PS}} \geq n$ performs reasonably well, but if $n_{\mathrm{PS}}$ drops below $n$, large deviations appear between the reweighted and the dedicated prediction.

Note that both the Thrust (for the region where three-jet production dominates) and the jet resolution for $n=2$, i.e. $y_{23}$ have been used to fit $\alpha_{S}\left(m_{Z}^{2}\right)$ [160], among other event-shape observables. The results of our final-state shower validations show that the required theory predictions can be generated with a reweighting, provided that $n_{\mathrm{PS}}$ is at least 3 or 4 .

\subsubsection{The initial-state dominated case: $W$-boson transverse momentum and jet resolutions in $\mathrm{pp} \rightarrow \mathrm{W}\left[\mathrm{e}^{-} v\right]$ events}

The second observable considered to validate our CSSHOWER and S-MC@NLO reweighting implementation is the $\mathrm{W}$-boson transverse-momentum $\left(p_{T}^{\mathrm{W}}\right)$ distribution in $13 \mathrm{TeV}$ protonproton collisions, that has already been used in Section 3.2 in the NLO case. The lepton cuts and the jet definition are unchanged. Also the definitions for constructing the uncertainty bands used there are kept the same. The only exception is the PDF band, where now we consider the $\mathrm{CT}_{14}$ set, which uses a Hessian error representation at a $90 \%$ confidence level [101]. ${ }^{6}$ Therefore the PDF error band will be larger than before, as it now corresponds to nearly two standard deviations instead of only one.

Considering a hadronic environment, initial-state emissions are present, which means that our reweighting factors now include PDF double ratios. In Fig. 3.7, we compare LOPS uncertainty bands for scale, $\alpha_{S}$ and PDF variations, including comparisons between reweighted and dedicated predictions for a varying maximum number of reweighted shower emissions $n_{\mathrm{PS}}$. Before discussing the bands, we observe that the tail of the $p_{T}^{\mathrm{W}}$ spectrum is not populated, in particular in comparison to Fig. 3.1. This is expected, as the LO configuration is constrained to $p_{T}^{\mathrm{W}}=0$, such that all other bins are filled through recoils against parton-

\footnotetext{
${ }^{6}$ The reason for switching from NNPDF 3.0 to $\mathrm{CT}_{14}$ PDFs is the strict positivity of the latter. The CSSHOWER rejects emissions when negative PDF values are involved, a behaviour which spoils the reweighting in regions where the original and the target PDF do not have the same sign, see Section 3.3.3. The deviations seem to be small in practical applications, but here we chose to establish closure in a clean context first.
} 

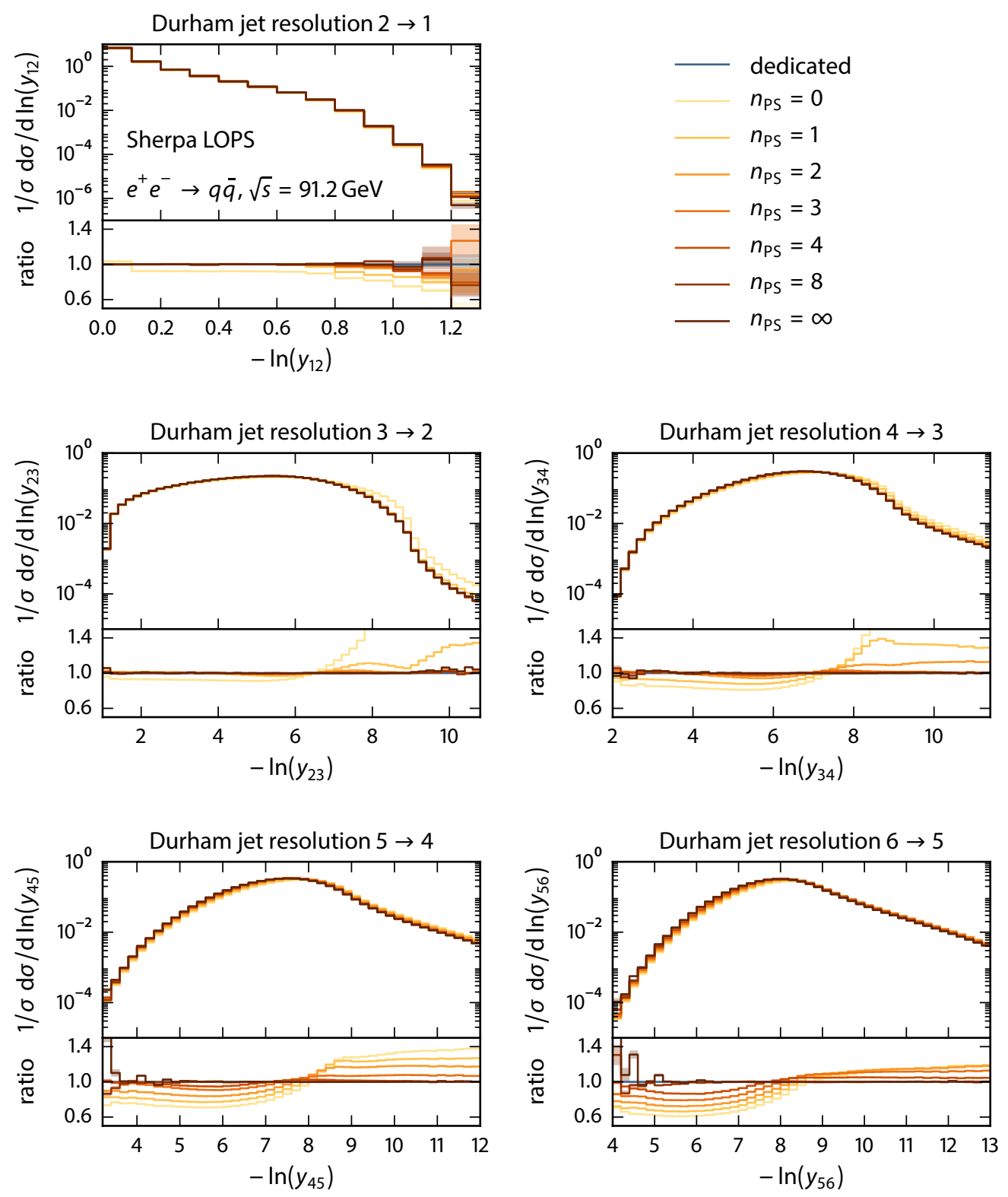

Figure 3.6.: Cross-closure test for the Durham jet resolutions at different multiplicities in dijet production for $\mathrm{e}^{+} \mathrm{e}^{-}$annihilation, reweighting $a_{S}\left(m_{Z}^{2}\right)$ up from 0.120 to 0.128 . A LO plus parton-shower calculation is used, and the maximum number of reweighted emissions $n_{\mathrm{PS}}$ is varied. The upper panels show the nominal distribution and the lower panels the ratio of the reweighted predictions to the dedicated prediction. The uncertainty bands give the statistical error, which is expected to grow for larger $n_{\text {PS. }}$. 

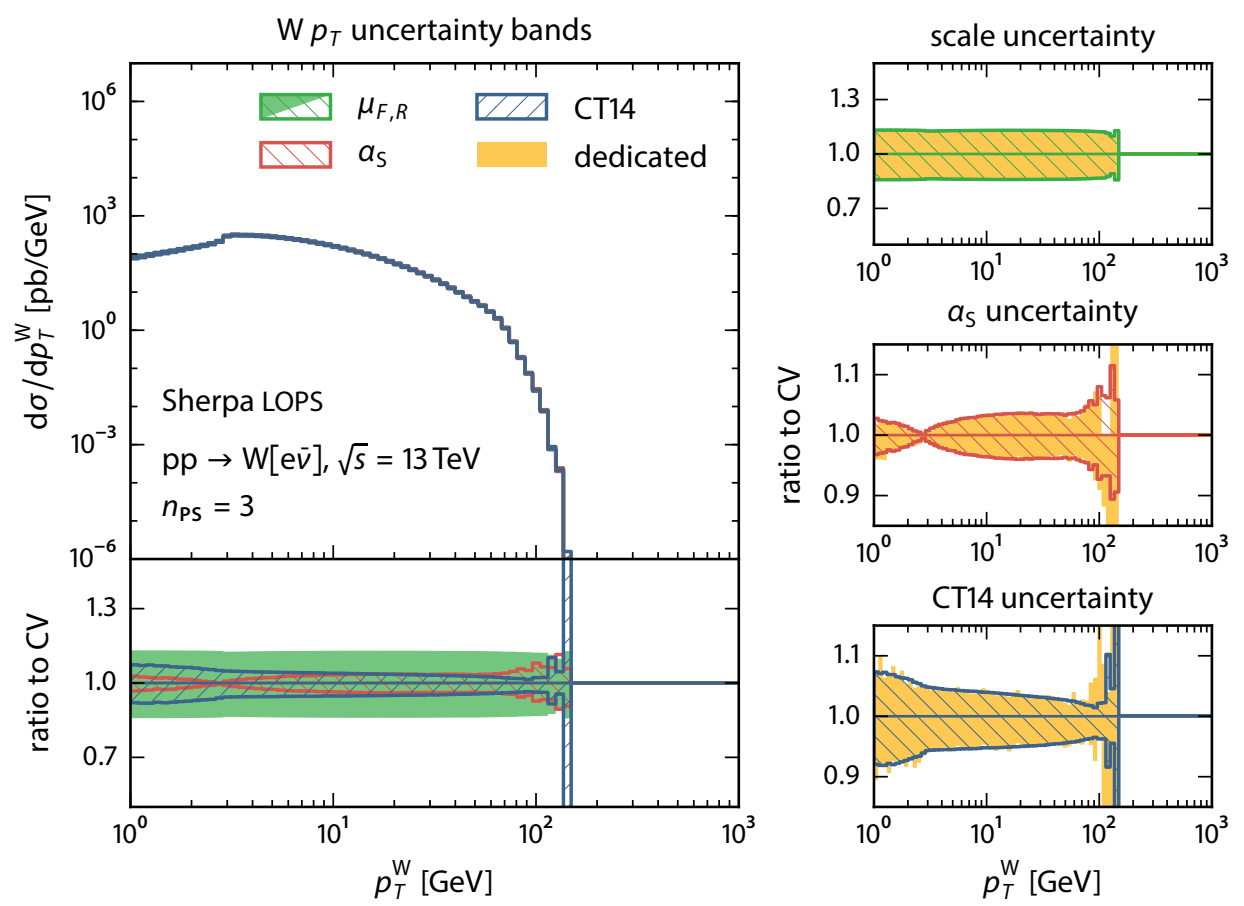

other maximum numbers of reweighted emissions $n_{\mathrm{PS}}$

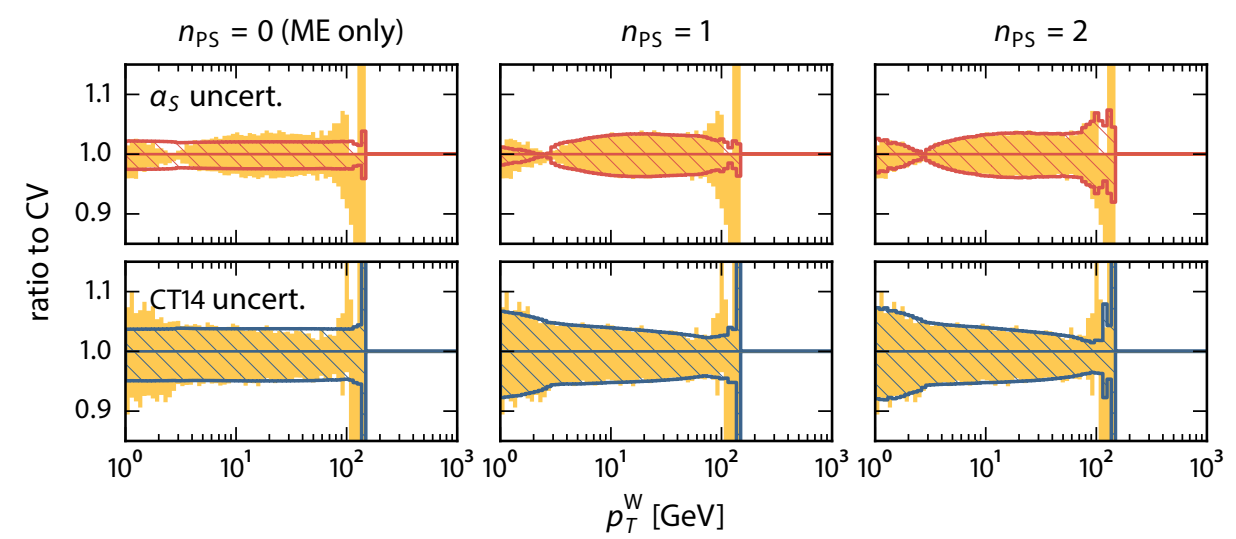

Figure 3.7.: The same as in Fig. 3.1, but for a LO plus parton-shower calculation. The uncertainty bands are calculated by reweighting the matrix element and up to $n_{\mathrm{PS}}$ shower emissions. In the upper four plots, $n_{\mathrm{PS}}=3$. In the lower plots, $n_{\mathrm{PS}}$ is varied for comparison. The scale uncertainties do not change with $n_{\mathrm{PS}}$ and are therefore not repeated. 
shower emissions only. However, the phase space of parton-shower emissions is restricted to the soft region, and therefore the $\mathrm{W}$ boson can not build up a large recoil.

We now turn to the scale uncertainty band-which is only due to factorisation scale variations, because the LO matrix element is independent of $\alpha_{S}$. Therefore, the band gives not a faithful estimate of the overall perturbative uncertainty. We also observe that the band is nearly flat. As we vary only the scales of the matrix-element calculation, the constant spread corresponds to the factorisation-scale uncertainty of the Born configuration for which $p_{T}^{\mathrm{W}}=0$, merely propagated to higher $p_{T}^{\mathrm{W}}$ bins through the parton shower, which is unaware of the scale variations. In the matrix-element reweighting, we can guarantee the same phase-space points as in the dedicated run, such that we see perfect agreement between dedicated and reweighted predictions. We therefore omit comparisons for different $n_{\mathrm{PS}}$ for the scale-uncertainty band.

Looking at the $\alpha_{S}$ uncertainty band, we can see that the envelope constricts at the position of the peak of the distributions. This reflects that the variation of $\alpha_{S}$ shifts the position of the peak, such that variations that are below the nominal distribution on the left side of the peak, are exceeding the nominal distribution on the right side, and vice versa. Comparing the reweighted prediction to the dedicated one, we find a flat band for $n_{\mathrm{PS}}=0$, corresponding to restricting the reweighting to the fixed-order matrix element. As the LO calculation is independent of $\alpha_{S}$, this only reflects the change of the PDFs, which are fitted to $\alpha_{S}\left(m_{\mathrm{Z}}^{2}\right)$. The reproduction of the shape of the $\alpha_{S}$ uncertainty improves a lot when reweighting up to one emission $\left(n_{\mathrm{PS}}=1\right)$, and slightly more when adding another emission on top $\left(n_{\mathrm{PS}}=2\right)$.

For the PDF uncertainty, we see that the reweighting with $n_{\mathrm{PS}}=0$ under-estimates it by at least $1-5 \%$ for small transverse momenta, and over-estimates it around the W mass. As for the $\alpha_{S}$ uncertainty, this improves for $n_{\mathrm{PS}}=1,2$.

The last depicted step, i.e. $n_{\mathrm{PS}}=3$, on the other hand, does not contribute further to the reproduction of the $\alpha_{S}$ and PDF uncertainties. No significant differences with respect to the $n_{\mathrm{PS}}=2$ case is observed. It can be concluded that it is sufficient to reweight only up to two emissions to reproduce the uncertainty bands for this observable.

This is to be expected, as the gauge boson recoils against the shower emissions and is therefore mostly affected by the few hardest emissions. These mainly originate from the incoming hard virtual partons, so the generally softer final-state emissions barely contribute. Although we do not reproduce this here, we confirmed this by entirely disabling final-state emissions, which showed no effect on the results.

In Fig. 3.8, we present the validation of NLOPS predictions for the $p_{T}^{\mathrm{W}}$ distribution. Overall, we get a similar picture as in the LOPS case, but with more faithful scale variations as the $\mu_{R}$ dependence is not present. They are smallest in the low $p_{T}^{\mathrm{W}}$ range, where the complete set of $\mathcal{O}\left(\alpha_{S}\right)$ corrections is present to the production process. For large $p_{T}^{\mathrm{W}}$, the uncertainty increases, because we fall back to a LO description again: Only the $2 \rightarrow 3$ matrix element still contributes. We have repeated the nominal distribution for LOPS for reference, which falls steeply for $p_{T}^{\mathrm{W}} \gtrsim m_{\mathrm{W}}$, whereas the NLOPS distribution continues to much higher values of $p_{T}^{\mathrm{W}}$.

To assess the quality of the reweighting, we consider again different settings for the 

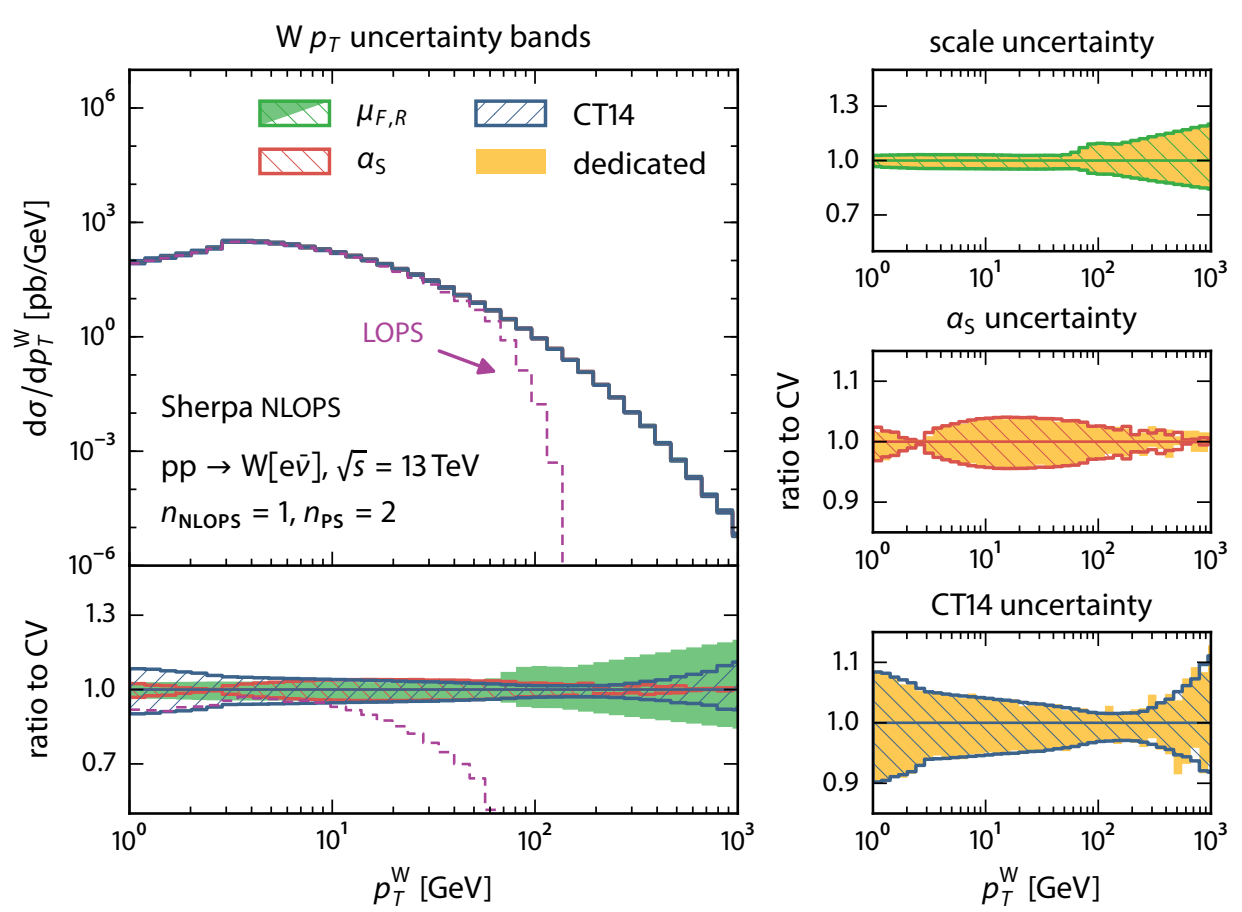

other maximum numbers of reweighted emissions $n_{\text {NLOPS }}, n_{\text {PS }}$

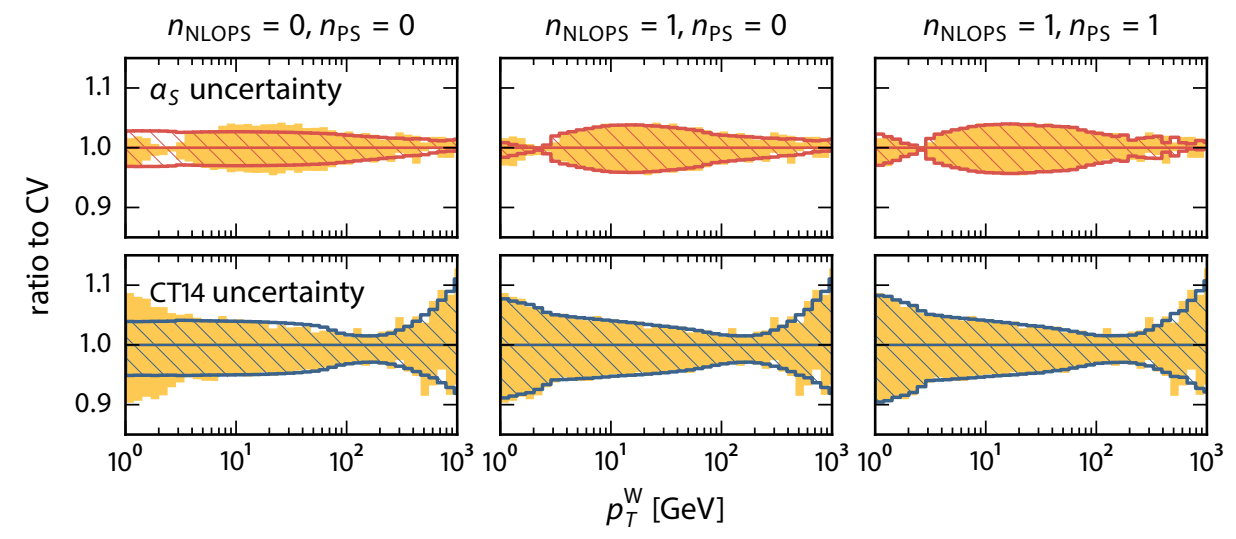

Figure 3.8.: The same as in Fig. 3.7, but for a NLO plus parton-shower calculation. The uncertainty bands are generated by reweighting the ME and a maximum number of emissions from the MC@NLO ( $\left.n_{\text {NLOPS }}\right)$ and the ordinary parton shower $\left(n_{\mathrm{PS}}\right) . n_{\text {NLOPS }}$ is constrained to 0 or 1, as the MC@NLO prescription only affects the first emission. In the upper four plots we consider $n_{\text {NLOPS }}=1$ and $n_{\text {PS }}=2$, thus up to three emissions are reweighted. In the lower plots, we consider variations of $n_{\text {NLOPS }}$ and $n_{\text {PS. }}$. 
parameters $n_{\mathrm{NLOPS}}$ and $n_{\mathrm{PS}}$. Assuming $n_{\mathrm{NLOPS}}=n_{\mathrm{PS}}=0$, only the scale variations of the hard process are considered and the parton-shower contribution to the $\mathcal{O}\left(\alpha_{S}\right)$ correction is not reweighted. Furthermore, we present results for $n_{\mathrm{NLOPS}}=1$ and $n_{\mathrm{PS}}=0,1,2$. With these settings, the $\mathcal{O}\left(\alpha_{S}\right)$ corrections get properly reweighted, and the number of subsequent shower emissions off the $\mathbb{S}$ - and $\mathbb{H}$-like events treated correctly is varied. We observe a saturation for reproducing the dedicated calculations at $n_{\mathrm{NLOPS}}+n_{\mathrm{PS}} \geq 2$, with no further improvement when $n_{\mathrm{PS}}$ is increased from 1 to 2 . This confirms the findings made when considering the LOPS setup in Fig. 3.7: The $p_{T}^{\mathrm{W}}$ distribution is dominated by the few hardest emissions.

To illustrate again the observable-dependence of choosing a sufficiently high $n_{\mathrm{PS}}$, we present jet resolutions at LOPS in Fig. 3.9. Again, jet resolutions $d_{n, n+1}$ give the jet distance parameter of the jet algorithm, in this case the anti- $k_{T}$ algorithm [107], at which an $(n+1)$ jet configuration is clustered into an $n$-jet configuration. For harder jets with $d_{n, n+1} \gtrsim 0.6$, we find a similar behaviour as for the jet resolution in $\mathrm{e}^{+} \mathrm{e}^{-}$annihilation in the previous section. When $n+1$ becomes larger than $n_{\mathrm{PS}}$, the reweighted result deviates away from the dedicated result. We included for reference the fully reweighted prediction for $n_{\mathrm{PS}}=\infty$, which always gives the correct result as it is expected to do.

\subsection{Multi-jet merging}

In this section we address the reweighting of multi-jet merged event generation runs. These approaches allow to combine LO or NLO QCD matrix elements of different multiplicity dressed with parton showers into inclusive samples, as discussed in Section 2.2.4.

To first approximation the reweighting as described in the previous sections can be used without change, only that the perturbative order $p$ is no longer a constant across the sample, but varies for each event, corresponding to the considered matrix-element parton multiplicity. However, there are also new algorithm-specific intricacies which complicate the dependence on the input parameters and need to be dealt with to allow for a consistent reweighting. In what follows we will detail the specifics of the reweighting procedure for LO and NLO multi-jet merging runs with SHERPA supplemented by an extensive validation of the implementation.

It should be emphasised here, parton-shower reweighting is vital when using modified input parameters in order to cancel the $Q_{\text {cut }}$ dependence to the accuracy of the parton shower. In case only the hard-process matrix element parameters get reweighted the leading dependence on $Q_{\text {cut }}$ still is cancelled but sub-leading contributions remain [33].

\section{Preliminaries}

Common to the LO and NLO merging techniques used in SHERPA, cf. [30, 33, 88, 125, 161], is the separation of the emission phase space into a soft and a hard region, defined through a suitable $m$-parton measure $Q_{m}$ and a separation criterion $Q_{c u t}$. For each parton configuration $\Phi_{m}$ with $Q_{m}>Q_{\text {cut }}$, a shower history that represents the event as a core process 

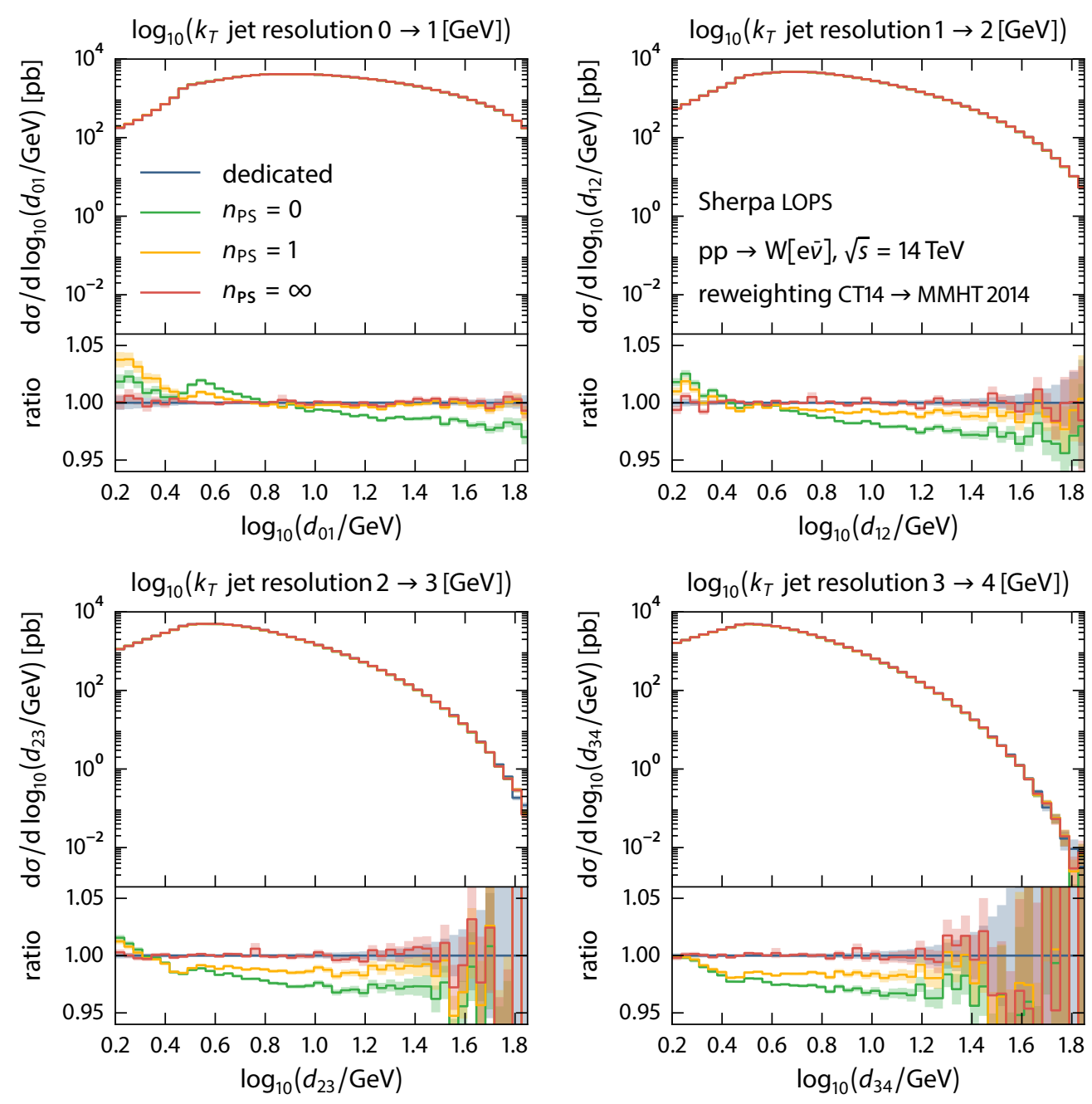

Figure 3.9.: Cross-closure test for the $k_{T}$ jet resolutions at different multiplicities in W production, reweighting from the CT14 PDF set to the MMHT2014 one. A LO plus parton-shower calculation is used, and the maximum number of reweighted emissions $n_{\mathrm{PS}}$ is varied. The upper panels show the nominal distribution and the lower panels the ratio of the reweighted predictions to the dedicated prediction. The uncertainty bands give the statistical error. 


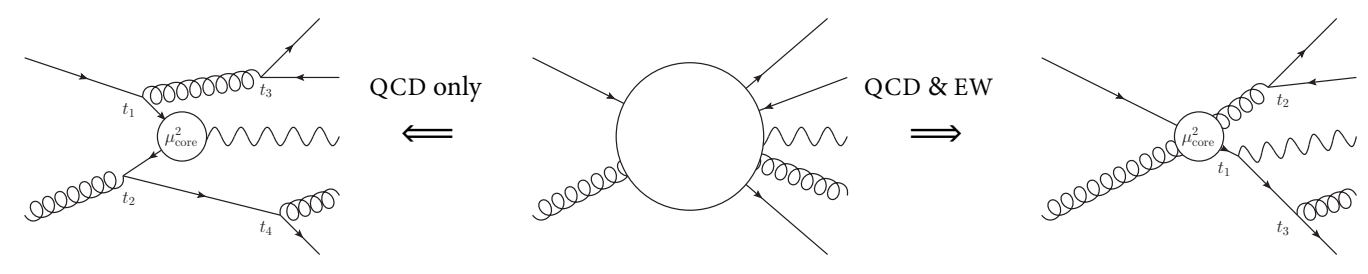

Figure 3.10.: Possible parton-shower histories of a $q g \rightarrow$ Zgqq' $\bar{q}^{\prime}$ matrix element allowing only QCD splittings (left) and also including electro-weak (EW) splittings (right).

with subsequent $1 \rightarrow 2$ shower splittings is probabilistically built through backward clustering. The resulting sequence of cluster steps is characterised by tuples $\left\{a_{i}, b_{i}, x_{a, i}, x_{b, i}, t_{i}\right\}$, recording the varying initial-state flavours and momentum fractions as well as the evolution variable of each splitting. We allow for both QCD and electro-weak splitting functions [30, 162] to identify such splitting processes and veto recombinations that would lead to the reduction of configurations which are not present in the matrix elements ${ }^{7}$. As an example, Figure 3.10 details possible cluster histories for a given $\mathrm{pp} \rightarrow \mathrm{Z}+4$ jets configuration, depending on its kinematics, allowing for QCD splittings only (left) or both QCD and electro-weak splittings (right).

The sequence $\left\{t_{i}\right\}$ of reconstructed emission scales may be either ordered or unordered, with an ordered history satisfying $t_{j}<t_{j-1}<\ldots<t_{1}<t_{0}=\mu_{F, \text { core }}^{2}$. The recombination probabilities in each clustering step are determined by the forward-splitting probabilities and are therefore dependent on the parton shower and its parameters and choices. This is reflected, step-by-step, in the addition of one factor of $\alpha_{S}$ (when appropriate) at the reconstructed splitting scale, a ratio of PDFs at the reconstructed initial flavours and their momentum fractions, and a Sudakov form factor describing the evolution of each step.

In the SHERPA implementations the $\alpha_{S}$ and PDF factors are added explicitly onto the respective matrix elements and can therefore be reweighted directly. The Sudakov form factor, on the other hand, is implemented through a vetoed truncated parton shower [30, 125]. The truncated shower itself, accounting for the possibility of soft parton-shower emissions between subsequent reconstructed hard emissions, i.e. with $t_{m}<t<t_{m-1}$ but $Q<Q_{\text {cut }}$, can be reweighted with the methods described in Section 3.3. If, however, an emission with $Q>Q_{\text {cut }}$ occurs the event is vetoed. Practically, this is accounted for through increasing $n_{\text {trials }}$ of the next accepted event by $n_{\text {trials }}$ of the vetoed event. Thus, $n_{\text {trials }}$ becomes dependent on the parton-shower parameters.

In unordered histories the $\left\{t_{i}\right\}$ sequence has at least one pair $t_{k} \geq t_{k-1}$. Such histories can be encountered in various configurations, e.g. when the last clustering step produces

\footnotetext{
${ }^{7}$ An example here is the interpretation of an $\mathrm{e}^{+} \mathrm{e}^{-} \rightarrow$ gdd configuration. Its matrix element does not contain terms/diagrams that allow the quark-antiquark pair to be clustered.
} 
a splitting scale larger than the nominal starting scale of the core process ${ }^{8}$, or the flavour structure only allows further clusterings at scales $t_{k-1}$ lower than the last identified one $t_{k}{ }^{9}$. As such configurations can not be generated by a strictly-ordered parton shower, for each unordered step neither the accompanying PDF ratio nor Sudakov form factor is therefore present in the calculation. More than one unordering in a cluster history of a given event is possible and in fact likely at high multiplicities. PDF ratios and Sudakov factors then of course only occur in the ordered subhistories in between the unorderings. For the sake of clarity and brevity we will omit unorderings from the discussion of the following subsections. Its implications to the algorithm, and therefore to the reweighting, are straightforward.

\subsection{The leading-order case: MEPS@LO}

We start the discussion with the simplest case, where all matrix elements used in the merging are given at leading order. A LO multijet-merged (MEPS@LO) calculation, with Born matrix elements at $\mathcal{O}\left(\alpha_{S}^{n+j}\right)$, containing $j$ additional partons relative to the core process, has the following structure

$$
\begin{aligned}
O & =\sum_{j=0}^{j_{\max }} \int \mathrm{d}_{j} \mathrm{~B}_{j}^{\text {merge }}\left(\Phi_{j}\right) \Theta\left(Q_{j}-Q_{\text {cut }}\right) \operatorname{PS}^{\mathrm{vt}}\left(O, \Phi_{j}\right) \\
& =\lim _{N \rightarrow \infty} \frac{1}{N_{\text {trials }}} \sum_{i=1}^{N} \sum_{j=0}^{j_{\max }} \mathrm{B}_{j}^{\text {merge }}\left(\Phi_{j, i}\right) \Theta\left(Q_{j}-Q_{\text {cut }}\right) \operatorname{PS}^{\mathrm{vt}}\left(O, \Phi_{j, i}\right) .
\end{aligned}
$$

Note that $\Phi_{j}$ here denotes the entire final-state phase space of the process, including all particles of the core process. As before, $Q_{j}$ is a suitable infrared-safe distance measure of $\Phi_{j}$. The $\Theta$-function thus realises a minimum separation of $Q_{c u t}$ and acts as an infrared regulator. $\mathrm{PS}^{\mathrm{vt}}$ is the vetoed truncated parton shower derived from Eq. (3.29). As the limit in the second line is well defined, it can be transposed with the summation over parton multiplicities. As the ingredient LO matrix elements need to incorporate the soft-collinear resummation properties of the parton shower, they have the following parameter dependences:

$$
\mathrm{B}_{j}^{\text {merge }}\left(\Phi_{j}\right) \equiv \mathrm{B}_{j}^{\text {merge }}\left(\Phi_{j} ; \alpha_{S}, f ; \mu_{R, \text { core }}, \mu_{F, \text { core }}, k_{\alpha_{S}}, k_{f} ;\left\{a_{i}, b_{i}, x_{a, i}, x_{b, i}, t_{i}\right\}\right) .
$$

The cluster steps $\left\{a_{i}, b_{i}, x_{a, i}, x_{b, i}, t_{i}\right\}$ denote the identified cluster history of the configuration $\Phi_{j}$, as discussed above. Therein, the $a_{i}, b_{i}$ are the possibly changing initial-state flavours, the $x_{a, i}, x_{b, i}$ their momentum fractions, and the $t_{i}$ are the reconstructed values of the parton-shower evolution variable at each splitting. Together with the $\alpha_{S}$ and PDF

\footnotetext{
${ }^{8}$ An example here is the interpretation of a gq $\rightarrow$ Zq configuration. In regions of large transverse momenta of the final-state parton its identified emission scale $t_{1}$ is larger than the starting scale $t_{0}$ of the core process $\mathrm{q} \overline{\mathrm{q}} \rightarrow \mathrm{Z}$, usually defined to be the invariant mass of the (virtual) $\mathrm{Z}$.

${ }^{9}$ An example here is the interpretation of a $\mathrm{e}^{+} \mathrm{e}^{-} \rightarrow$ d $\bar{d} u \bar{u}$ configuration. In a first step there are only two choices to cluster, resulting in an identified emission scale $t_{2}$. There now is only a finite region in phase space where the gluon can be clustered with scale $t_{1}<t_{2}$.
} 
scale prefactors $k_{\alpha_{S}}, k_{f}$ of the parton shower, the cluster steps relate $\mathrm{B}_{j}^{\text {merge }}$ to the scale- and PDF-stripped Born matrix element $\mathrm{B}_{j}^{\prime}$ encountered in Section 3.1.1,

$$
\begin{aligned}
& \mathrm{B}_{j}^{\text {merge }}\left(\Phi_{j} ; \alpha_{S}, f ; \mu_{R, \text { core }}, \mu_{F, \text { core }}, k_{\alpha_{S}}, k_{f} ;\left\{a_{i}, b_{i}, x_{a, i}, x_{b, i}, t_{i}\right\}\right) \\
& =\prod_{i=1}^{j} \frac{f_{a_{i}}\left(x_{a, i}, k_{f} t_{i}\right)}{f_{a_{i-1}}\left(x_{a, i-1}, k_{f} t_{i}\right)} f_{a_{0}}\left(x_{a, 0}, \mu_{F, \text { core }}^{2}\right) \prod_{i=1}^{j} \frac{f_{b_{i}}\left(x_{b, i}, k_{f} t_{i}\right)}{f_{b_{i-1}}\left(x_{b, i-1}, k_{f} t_{i}\right)} f_{b_{0}}\left(x_{b, 0}, \mu_{F, \text { core }}^{2}\right) \\
& \quad \times \alpha_{S}^{n+j}\left(\mu_{R}^{2}\right) \mathrm{B}_{j}^{\prime}\left(\Phi_{j}\right) .
\end{aligned}
$$

In this notation, the core scale is $t_{0}=\mu_{F, \text { core }}^{2}$, it is therefore not multiplied by the prefactors of the parton shower. The partonic momentum fractions of the core process are $x_{a, 0}, x_{b, 0}$.

The scales of each single $\alpha_{S}$ within the cluster history vary, but an effective global renormalisation scale can be defined through

$$
\alpha_{S}^{n+j}\left(\mu_{R}^{2}\right)=\alpha_{S}^{n+e}\left(\mu_{R, \text { core }}^{2}\right) \prod_{i=1}^{j} \alpha_{S}^{1-\epsilon_{i}}\left(k_{\alpha_{S}} t_{i}\right)
$$

where $\epsilon_{i}=0$ if the identified splitting process at branching $i$ is of QCD-type, and 1 otherwise, $e=\sum_{i=1}^{j} \epsilon_{i}$. To consistently vary the $\mu_{R}$ scale, we consider variations of the splitting scales $t_{i}$ and the core scale $\mu_{R \text {,core }}$ on the right-hand side by a common factor, solving for the prefactor of the effective $\mu_{R}$ to be used in the matrix-element calculation. Thus, while up to NLO accuracy $\mu_{R}$ is varied by the same common factor, the full solution of this procedure results in slightly larger variations of the effective renormalisation scale.

Apart from the Sudakov form factors the soft-collinear structure of the $\mathrm{B}_{j}^{\text {merge }}$ is now identical to the emission of $j$ partons off a $\mathrm{B}_{0}$ configuration with the parton shower described in Section 3.3. In the case of final-state splittings, the ratio of PDFs is simply unity as neither the partonic $x_{a / b, i}$ and $x_{a / b, i-1}$ nor the initial-state flavours $a_{i}, b_{i}$ and $a_{i-1}, b_{i-1}$ differ. In principle, with every ratio of PDFs there is also a ratio of flux factors. However, all such factors cancel except for the outermost ones, corresponding to $\Phi_{j}$ and, hence, are regarded as part of $\mathrm{B}_{j}^{\prime}$.

Changing the scales $\mu_{R, \text { core }} \rightarrow \tilde{\mu}_{R \text {,core }} \mu_{F, \text { core }} \rightarrow \tilde{\mu}_{F, \text { core }}, k_{\alpha_{S}} \rightarrow \tilde{k}_{\alpha_{S}}, k_{f} \rightarrow \tilde{k}_{f}$ as well as $\alpha_{S} \rightarrow \tilde{\alpha}_{S}$ and $f \rightarrow \tilde{f}$ results in

$$
\begin{aligned}
& \mathrm{B}_{j}^{\text {merge }}\left(\Phi_{j} ; \tilde{\alpha}_{S}, \tilde{f}_{\tilde{\mu}} \tilde{\mu}_{R, \text { core }}, \tilde{\mu}_{F, \text { core }}, \tilde{k}_{\alpha_{S}}, \tilde{k}_{f} ;\left\{a_{i}, b_{i}, x_{a, i}, x_{b, i}, t_{i}\right\}\right) \\
& =\prod_{i=1}^{j} \frac{\tilde{f}_{a_{i}}\left(x_{a, i}, \tilde{k}_{f} t_{i}\right)}{\tilde{f}_{a_{i-1}}\left(x_{a, i-1}, \tilde{k}_{f} t_{i}\right)} \tilde{f}_{a_{0}}\left(x_{a, 0}, \tilde{\mu}_{F, \text { core }}^{2}\right) \prod_{i=1}^{j} \frac{\tilde{f}_{b_{i}}\left(x_{b, i}, \tilde{k}_{f} t_{i}\right)}{\tilde{f}_{b_{i-1}}\left(x_{b, i-1}, \tilde{k}_{f} t_{i}\right)} \tilde{f}_{b_{0}}\left(x_{b, 0}, \tilde{\mu}_{F, \text { core }}^{2}\right) \\
& \quad \times \tilde{\alpha}_{S}^{n+j}\left(\tilde{\mu}_{R}^{2}\right) \mathrm{B}_{j}^{\prime}\left(\Phi_{j}\right) .
\end{aligned}
$$

The scale $\tilde{\mu}_{R}^{2}$ is now calculated from Eq. (3.44) using $\tilde{\mu}_{R \text {,core }}^{2}$ and $\tilde{k}_{\alpha_{S}}$ as input. 
Equation (3.45) describes what happens to the matrix-element part of a multi-jet merged calculation. This leaves the vetoed truncated shower PS ${ }^{\mathrm{vt}}$. While the truncated and standard shower part is described in Section 3.3, the vetoed shower leads to vetoed events. As vetoed events correspond to events whose weights have been set to zero, their description is equivalent to increasing the number of trials, $n_{\text {trials }}$, by one. Thus, when varying the parameters of the parton shower, also the probabilities of vetoing events are changed. Consequently, $n_{\text {trials }}$ acquires a dependence on the parameters of the variation. Thus, now explicitly stating the dependence on the shower starting scale $\mu_{Q}^{2}$ and the merging scale $Q_{\text {cut }}$,

$$
\begin{aligned}
& n_{\text {trials }}\left(\mu_{Q}^{2} ; Q_{\text {cut }}^{2} ;\left\{a_{i}, b_{i}, x_{a, i}, x_{b, i}, t_{i}\right\} ; \tilde{\alpha}_{S}, \tilde{f} ; \tilde{k}_{\alpha_{S}}, \tilde{k}_{f}\right) \\
& =\frac{1-\Delta_{n+j}\left(t_{\mathrm{IR}}, \mu_{Q}^{2} ; Q_{\mathrm{cut}}^{2} ; \tilde{\alpha}_{S}, \tilde{f} ; \tilde{k}_{\alpha_{S}}, \tilde{k}_{f} ;\left\{a_{i}, b_{i}, x_{a, i}, x_{b, i}, t_{i}\right\}\right)}{1-\Delta_{n+j}\left(t_{\mathrm{IR}}, \mu_{Q}^{2} ; Q_{\mathrm{cut}}^{2} ; \alpha_{S}, f ; k_{\alpha_{S}}, k_{f} ;\left\{a_{i}, b_{i}, x_{a, i}, x_{b, i}, t_{i}\right\}\right)},
\end{aligned}
$$

where

$$
\begin{aligned}
& \Delta_{n+j}\left(t_{\mathrm{IR}}, \mu_{Q}^{2} ; Q_{\mathrm{cut}}^{2} ; \alpha_{S}, f ; k_{\alpha_{S}}, k_{f} ;\left\{a_{i}, b_{i}, x_{a, i}, x_{b, i}, t_{i}\right\}\right) \\
& =\prod_{i=1}^{j} \exp \left\{-\int_{t_{i}}^{t_{i-1}} \mathrm{~d} \Phi_{1} \mathrm{~K}_{n+i}\left(\Phi_{1} ; k_{\alpha_{S}}, k_{f} ; \alpha_{S}, f\right) \Theta\left(Q_{n+i}>Q_{\mathrm{cut}}\right)\right\} .
\end{aligned}
$$

Thus, $n_{\text {trials }}$ corresponds to the survival probability between the unfolding of preexisting splittings when evolving from the $n$-parton core configuration to the $(n+j)$-parton configuration. When changing the parameters of the simulation, the probability of the truncated shower to emit a parton with $Q>Q_{\text {cut }}$ must be re-evaluated following the substitutions $k_{\alpha_{S}} \rightarrow \tilde{k}_{\alpha_{S}}, k_{f} \rightarrow \tilde{k}_{f}, \alpha_{S} \rightarrow \tilde{\alpha}_{S}$ and $f \rightarrow \tilde{f}$ using the methods of Section 3.3. Note, all emissions produced by the truncated shower prior to the one that triggers the veto need to be reweighted as they impact the initial conditions for that emission.

\subsection{The next-to-leading-order case: MEPS@NLO}

The merging of multi-jet matrix elements at NLO accuracy (MEPS@NLO) proceeds schematically similar as in the LO case. The input quantity is now the NLOPS matched $(n+j)$-parton 
configuration, thus

$$
\begin{aligned}
&\langle O\rangle^{\mathrm{MEPS} @ N L O}= \sum_{j=0}^{j_{\max }}\left[\int \mathrm{d} \Phi_{j} \overline{\mathrm{B}}_{j}^{\text {merge }}\left(\Phi_{j}\right) \Theta\left(Q_{j}-Q_{\mathrm{cut}}\right) \operatorname{NLOPS}^{\mathrm{v}}\left(O, \Phi_{j}\right)\right. \\
&\left.+\int \mathrm{d} \Phi_{j+1} \mathrm{H}_{A, j}^{\text {merge }}\left(\Phi_{j+1}, Q_{\mathrm{cut}}\right) \operatorname{PS}^{\mathrm{vt}}\left(O, \Phi_{j+1}\right)\right] \\
&=\lim _{N \rightarrow \infty} \frac{1}{N_{\text {trials }}}\left\{\sum_{i=1}^{N_{\mathrm{S}}} \sum_{j=0}^{j_{\max }} \overline{\mathrm{B}}_{j}^{\mathrm{merge}}\left(\Phi_{j, i}\right) \Theta\left(Q_{j}-Q_{\mathrm{cut}}\right) \operatorname{NLOPS}^{\mathrm{v}}\left(O, \Phi_{j, i}\right)\right. \\
&\left.+\sum_{i=1}^{N_{\mathrm{H}}} \sum_{j=0}^{j_{\max }} \mathrm{H}_{A, j}^{\mathrm{merge}}\left(\Phi_{j+1, i}, Q_{\mathrm{cut}}\right) \operatorname{PS}^{\mathrm{vt}}\left(O, \Phi_{j+1, i}\right)\right\}
\end{aligned}
$$

keeping the notation of Eqs. (3.35) and (3.41). For the $\mathbb{S}$-events the same $\Theta$-function of Eq. (3.41) is used as an infrared regulator and the S-MC@NLO parton shower of Eq. (3.40) is replaced by its vetoed version. It only matches the softest emission in $t$ and, thus, does not generate truncated emissions. These are added dressing it with additional emissions through the standard parton shower. As all ingredients of $\overline{\mathrm{B}}_{j}^{\text {merge }}$ are evaluated at the same phase-space point $\Phi_{j}$, they share a common cluster history $\left\{a_{i}, b_{i}, x_{a, i}, x_{b, i}, t_{i}\right\}$. Hence, again suppressing any further $Q_{\text {cut }}$-dependence which is not varied,

$$
\overline{\mathrm{B}}_{j}^{\text {merge }}\left(\Phi_{j}\right) \equiv \overline{\mathrm{B}}_{j}^{\text {merge }}\left(\Phi_{j} ; \alpha_{S}, f ; \mu_{R, \text { core }}, \mu_{F, \text { core }}, k_{\alpha_{S}}, k_{f} ;\left\{a_{i}, b_{i}, x_{a, i}, x_{b, i}, t_{i}\right\}\right) .
$$

This transforms under the replacements $k_{\alpha_{S}} \rightarrow \tilde{k}_{\alpha_{S}}, k_{f} \rightarrow \tilde{k}_{f}, \alpha_{S} \rightarrow \tilde{\alpha}_{S}$ and $f \rightarrow \tilde{f}$ in the following way,

$$
\begin{gathered}
\overline{\mathrm{B}}_{j}^{\text {merge }}\left(\Phi_{j ;} ; \tilde{\alpha}_{S}, \tilde{f} ; \tilde{\mu}_{R, \text { core }}, \tilde{\mu}_{F, \text { core }}, \tilde{k}_{\alpha_{S}}, \tilde{k}_{f} ;\left\{a_{i}, b_{i}, x_{a, i}, x_{b, i}, t_{i}\right\}\right) \\
=\prod_{i=1}^{j} \frac{\tilde{f}_{a_{i}}\left(x_{a, i}, \tilde{k}_{f} t_{i}\right)}{\tilde{f}_{a_{i-1}}\left(x_{a, i-1}, \tilde{k}_{f} t_{i}\right)} \tilde{f}_{a_{0}}\left(x_{a, 0}, \tilde{\mu}_{F, \text { core }}^{2}\right) \prod_{i=1}^{j} \frac{\tilde{f}_{b_{i}}\left(x_{b, i}, \tilde{k}_{f} t_{i}\right)}{\tilde{f}_{b_{i-1}}\left(x_{b, i-1}, \tilde{k}_{f} t_{i}\right)} \tilde{f}_{b_{0}}\left(x_{b, 0}, \tilde{\mu}_{F, \text { core }}^{2}\right) \\
\times \tilde{\alpha}_{S}^{n+j}\left(\tilde{\mu}_{R}^{2}\right)\left[\mathrm{B}_{j}^{\prime}\left(\Phi_{j}\right)+\tilde{\alpha}_{S}\left(\tilde{\mu}_{R}^{2}\right)\left(\mathrm{VI}_{j}^{\prime}\left(\Phi_{j}\right)+c_{R, j}^{\prime(0)} l_{R}+\frac{1}{2} c_{R, j}^{\prime(1)} l_{R}^{2}\right)\right. \\
+\tilde{\alpha}_{S}\left(\tilde{\mu}_{R}^{2}\right) \int \mathrm{d} x_{a / b}^{\prime} \mathrm{KP}_{j}^{\prime}\left(\Phi_{j}, x_{a / b}^{\prime} ; \tilde{f}_{;} \tilde{\mu}_{F, \text { core }}\right) \\
\left.+\tilde{\alpha}_{S}\left(\tilde{\mu}_{R}^{2}\right) \sum_{k} \int \mathrm{d} \Phi_{1}^{k}\left(\mathrm{D}_{A, k}^{\prime}-\mathrm{D}_{S, k}^{\prime}\right)\left(\Phi_{j} \cdot \Phi_{1}^{k}\right)\right] \\
-\sum_{i=1}^{j} \frac{\tilde{\alpha}_{S}\left(\tilde{\mu}_{R}^{2}\right)}{2 \pi} \log \frac{t_{i-1}\left(\sum_{c=q, g} \int \frac{\mathrm{d} x_{a, i}^{\prime}}{x_{a, i}^{\prime}} P_{a c}\left(x_{a, i}^{\prime}\right) \tilde{f}_{c}\left(\frac{x_{a, i}}{x_{a, i}^{\prime}}, \tilde{k}_{f} t_{i}\right)\right.}{+} \\
\left.+\sum_{d=q, g} \int \frac{\mathrm{d} x_{b, i}^{\prime}}{x_{b, i}^{\prime}} P_{b d}\left(x_{b, i}^{\prime}\right) \tilde{f}_{d}\left(\frac{x_{b, i}}{x_{b, i}^{\prime}}, \tilde{k}_{f} t_{i}\right)\right) \tilde{\alpha}_{S}^{n+j}\left(\tilde{\mu}_{R}^{2}\right) \mathrm{B}_{j}^{\prime}\left(\Phi_{j}\right) .
\end{gathered}
$$


In addition to the transformation properties of the $\bar{B}$-function of Eq. (3.36), supplemented with the PDF ratios already encountered in the LO case, additional terms appear. They subtract the $\mathcal{O}\left(\alpha_{S}\right)$ expansion of these ratios, in order to retain the NLO accuracy of the merged calculation. Again, please note that $t_{0}=\mu_{F, \text { core }}^{2}$.

The $\mathbb{H}$-events do not have a homogeneous phase-space point. Their constituent realemission matrix elements are defined on $\Phi_{j+1}$, while each subtraction term $\mathrm{D}_{A, k}$ has its own projection on a phase-space point $\Phi_{j}^{k}$. Thus,

$$
\begin{aligned}
& \mathrm{H}_{A, j}^{\text {merge }}\left(\Phi_{j+1}, Q_{\text {cut }}\right) \\
& \equiv \mathrm{R}_{j}^{\text {merge }}\left(\Phi_{j+1} ; \alpha_{S}, f ; \mu_{R, \text { core }}, \mu_{F, \text { core }}, k_{\alpha_{S}}, k_{f} ;\left\{a_{i}, b_{i}, x_{a, i}, x_{b, i}, t_{i}\right\}\right) \Theta\left(Q_{j}-Q_{\text {cut }}\right) \\
& \quad-\sum_{k} \mathrm{D}_{A, k, j}^{\text {merge }}\left(\Phi_{j}^{k} \cdot \Phi_{1}^{k} ; \alpha_{S}, f ; \mu_{R, \text { core }, k}, \mu_{F, \text { core }, k}, k_{\alpha_{S}}, k_{f} ;\right. \\
& \left.\left\{a_{i, k}, b_{i, k}, x_{a, i, k}, x_{b, i, k}, t_{i, k}\right\}\right) \Theta\left(Q_{j}^{k}-Q_{\text {cut }}\right),
\end{aligned}
$$

wherein both $\mathrm{R}_{j}^{\text {merge }}$ and the $\mathrm{D}_{A, k, j}^{\text {merge }}$ separately transform as the LO counterpart $\mathrm{B}_{j}^{\text {merge }}$. While the measure $Q$ on $\Phi_{j+1}$ of $\mathrm{R}_{j}^{\text {merge }}$ is defined to act on the underlying $\Phi_{j}$, after the first cluster step reducing the real-emission configuration to a Born configuration, it is defined directly on each $\Phi_{j}^{k}$ in each $\mathrm{D}_{A, k}$. Infrared safety is guaranteed through the infrared safety of their phase-space maps, the clustering algorithm and the measure $Q$.

Finally, we consider MEPS@NLO calculations that are extended by additional LO matrix elements through the MENLOPS scheme, as described in Section 2.2.4. We denote the total number of jets as $j_{\max }$, and the number of jets described to NLO as $j_{\max }^{\mathrm{NLO}}$,

$$
\begin{aligned}
& \langle O\rangle^{\text {MEPS@NLO+MENLOPS }} \\
& =\sum_{j=0}^{j_{\text {max }}^{\mathrm{NLO}}}\left[\int \mathrm{d} \Phi_{j} \overline{\mathrm{B}}_{j}^{\text {merge }}\left(\Phi_{j}\right) \operatorname{NLOPS}^{\mathrm{v}}\left(O, \Phi_{j}\right)\right. \\
& \left.+\int \mathrm{d} \Phi_{j+1} \mathrm{H}_{A, j}^{\mathrm{merge}}\left(\Phi_{j+1}\right) \operatorname{PS}^{\mathrm{vt}}\left(O, \Phi_{j+1}\right)\right] \\
& +\sum_{j=j_{\max }^{\mathrm{LNO}}+1}^{j_{\max }} \int \mathrm{d} \Phi_{j} k_{j_{\max }^{\mathrm{NLO}}}\left(\Phi_{j_{\max }^{\mathrm{NLO}+1}}\left(\Phi_{j}\right)\right) \mathrm{B}_{j}^{\mathrm{merge}}\left(\Phi_{j}\right) \operatorname{PS}^{\mathrm{vt}}\left(O, \Phi_{j}\right) \\
& =\lim _{N \rightarrow \infty} \frac{1}{N_{\text {trials }}}\left\{\sum_{i=1}^{N_{\mathrm{S}}} \sum_{j=0}^{j_{\text {max }}^{\text {NLO }}} \overline{\mathrm{B}}_{j}^{\text {merge }}\left(\Phi_{j, i}\right) \operatorname{NLOPS}^{\mathrm{v}}\left(O, \Phi_{j, i}\right)\right. \\
& +\sum_{i=1}^{N_{\mathrm{H}}} \sum_{j=0}^{\mathrm{NLO}} \mathrm{H}_{A, j}^{\mathrm{merge}}\left(\Phi_{j+1, i}\right) \operatorname{PS}^{\mathrm{vt}}\left(O, \Phi_{j+1, i}\right) \\
& \left.+\sum_{i=1}^{N_{\mathrm{LO}}} \sum_{j=j_{\max }^{\mathrm{NLO}+1}}^{j_{\max }} k_{j_{\max }^{\mathrm{NLO}}}\left(\Phi_{j_{\max }^{\mathrm{NLO}+1}}\left(\Phi_{j}\right)\right) \mathrm{B}_{j}^{\mathrm{merge}}\left(\Phi_{j, i}\right) \operatorname{PS}^{\mathrm{vt}}\left(O, \Phi_{j, i}\right)\right\},
\end{aligned}
$$

with $N=N_{\mathbb{S}}+N_{\mathbb{H}}+N_{\mathrm{LO}}$. A differential $K$-factor $k_{j_{\max }^{\mathrm{NLO}}}$ is applied to the higher-multiplicity 
leading-order matrix elements in order to facilitate a smooth transition across $Q_{\text {cut }}$. It has the form

$$
k_{m}\left(\Phi_{m+1}\right)=\frac{\overline{\mathrm{B}}_{m}\left(\Phi_{m}\right)}{\mathrm{B}_{m}\left(\Phi_{m}\right)}\left(1-\frac{\mathrm{H}_{A, m}\left(\Phi_{m+1}\right)}{\mathrm{R}_{m}\left(\Phi_{m+1}\right)}\right)+\frac{\mathrm{H}_{A, m}\left(\Phi_{m+1}\right)}{\mathrm{R}_{m}\left(\Phi_{m+1}\right)}
$$

and therefore moulds the $\mathrm{B}_{j_{\max }^{\mathrm{NLO}}+1}$ into the same form as the $\mathrm{H}_{A, j_{\max }^{\mathrm{NLO}}}$ it is replacing. The projection $\Phi_{j_{\max } \mathrm{NLO}}\left(\Phi_{j}\right)$ for $j>j_{\max }^{\mathrm{NLO}}+1$ is defined through its cluster history, as is $\Phi_{m}\left(\Phi_{m+1}\right)$ inside $k_{m}$ itself. When we change now the parameters of the calculation, $\alpha_{S} \rightarrow \tilde{\alpha}_{S}, f \rightarrow \tilde{f}$, $\mu_{R} \rightarrow \tilde{\mu}_{R}$ and $\mu_{F} \rightarrow \tilde{\mu}_{F}, k_{j_{\max }^{\mathrm{N} L O}}$ transforms as a composite object in terms of its constituents, cf. Sections 3.1.1, 3.1.2 and 3.3. The scales are set directly by the $\mathrm{B}_{j}^{\text {merge }}$ process. Of course, in the interest of decreased computational costs one may decide to choose $k_{m} \equiv 1$ throughout at the cost of larger merging systematics. Similarly, if an electroweak cluster history leads to a changed signature in $\Phi_{m+1}, k_{m} \equiv 1$ is chosen.

\subsection{Closure tests for multi-jet merged calculations}

The reweighting for multi-jet merged calculations as discussed in the previous sections has been implemented within SHERPA with the CSSHOWER for leading-order matrix elements(MEPS@LO), next-to-leading order matrix elements (MEPS@NLO) and next-toleading-order matrix elements with additional leading-order ones on top (MENLOPS). For the validation, we again perform closure tests between reweighted and dedicated predictions for the transverse momentum of the W-boson in Figs. 3.11 to 3.13. Again, the definitions for the scale uncertainty band are listed in Table 2.1, and the lepton cuts and the jet definition are the same as in Section 3.2. In addition, we employ a merging cut of $Q_{\text {cut }}=20 \mathrm{GeV}$.

For the MEPS@LO validation in Fig. 3.11, we combine LO matrix elements for o-, 1- and 2-jet multiplicities, obtained from Comix [70]. We can observe that we populate a much larger phase space than for a mere LOPS calculation in terms of $p_{T}^{\mathrm{W}}$. Below the merging cut (i.e. $p_{T}^{\mathrm{W}} \lesssim 20 \mathrm{GeV}$ ), the scale uncertainty band is equal to the one of the LOPS calculation. For higher $p_{T}^{\mathrm{W}}$, the scale uncertainty increases corresponding to the larger uncertainty of the higher-multiplicity matrix elements, that contribute renormalisation scale uncertainties.

In Fig. 3.12, we consider the MENLOPS case. We combine an NLO matrix element for the o-jet multiplicities with LO matrix elements for the 1- and 2-jet multiplicities. The scale uncertainty for low $p_{T}^{\mathrm{W}}$ values now features the reduced scale uncertainty, that we already have seen in the NLOPS validation.

The same is true in the MEPS@NLO case depicted in 3.13. A direct comparison of the scale uncertainties to the MENLOPS case is not straightforward though, as we combine NLO matrix elements for the 0 - and the 1-jet multiplicity, where the virtual amplitudes are obtained from ВцаскНат. Hence, the 2-jet multiplicity is described at leading order through the 1-jet H-events. As such, the set-up is not a simple "upgrade" from our MENLOPS calculation.

For MEPS@NLO, we have also added the nominal result for the NLOPS calculation for 

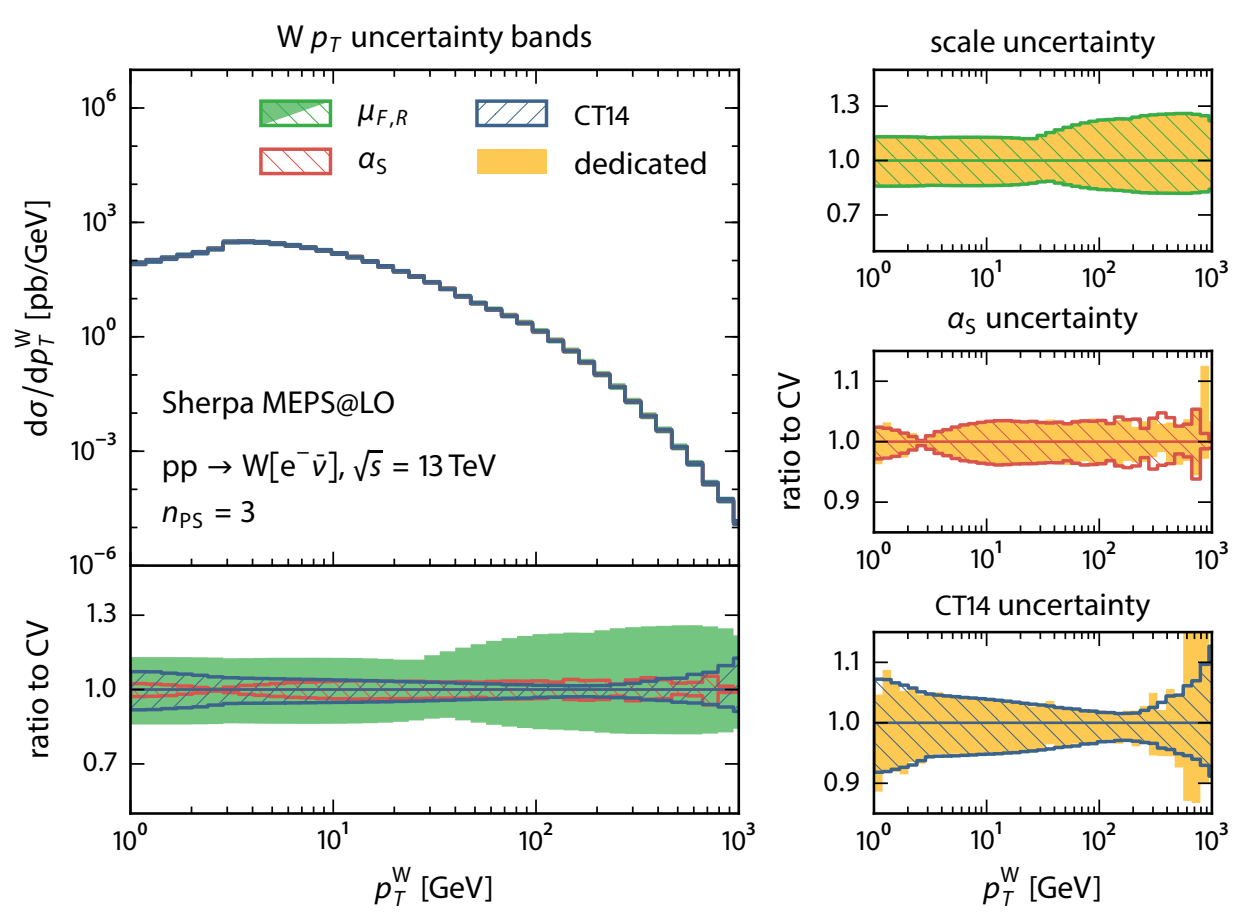

other maximum numbers of reweighted emissions $n_{\mathrm{PS}}$

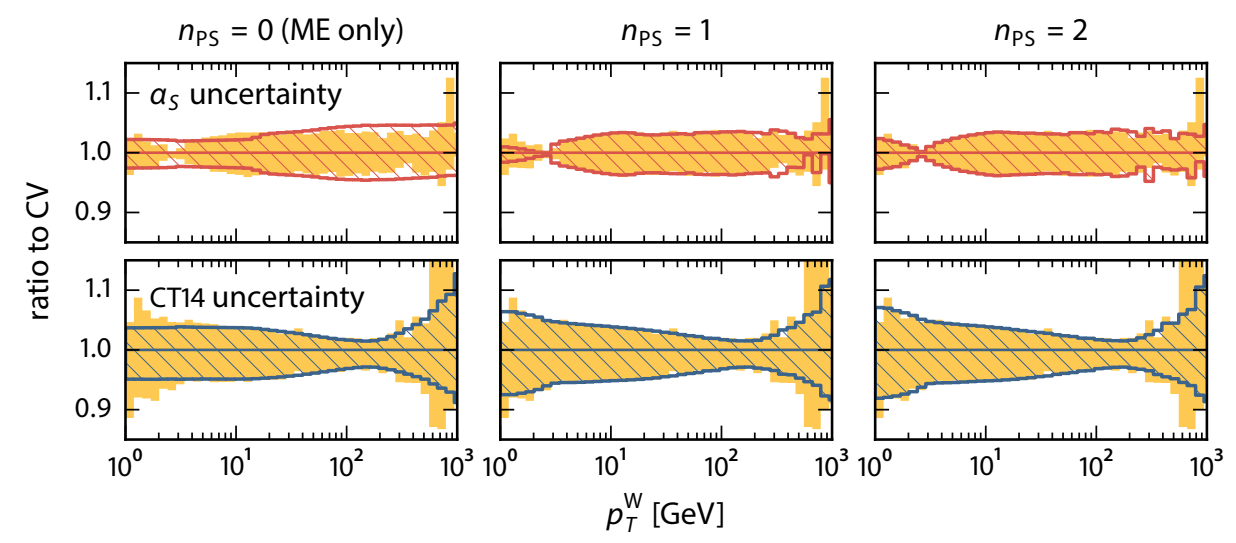

Figure 3.11.: The same as in Fig. 3.8, but for a multi-jet merged generation with LO matrix elements for 0-, 1- and 2-jet multiplicities. The uncertainty bands are calculated by reweighting the matrix element and a maximum number of emissions $n_{\mathrm{PS}}$ of parton-shower emissions. In the upper four plots, $n_{\mathrm{PS}}=3$, thus up to three emissions are reweighted. In the lower plots, $n_{\mathrm{PS}}$ is varied for comparison. Again, we find a saturation when reproducing dedicated calculations for $n_{\mathrm{PS}} \geq 2$, with no further improvement when $n_{\mathrm{PS}}$ is increased from 2 to 3 . 

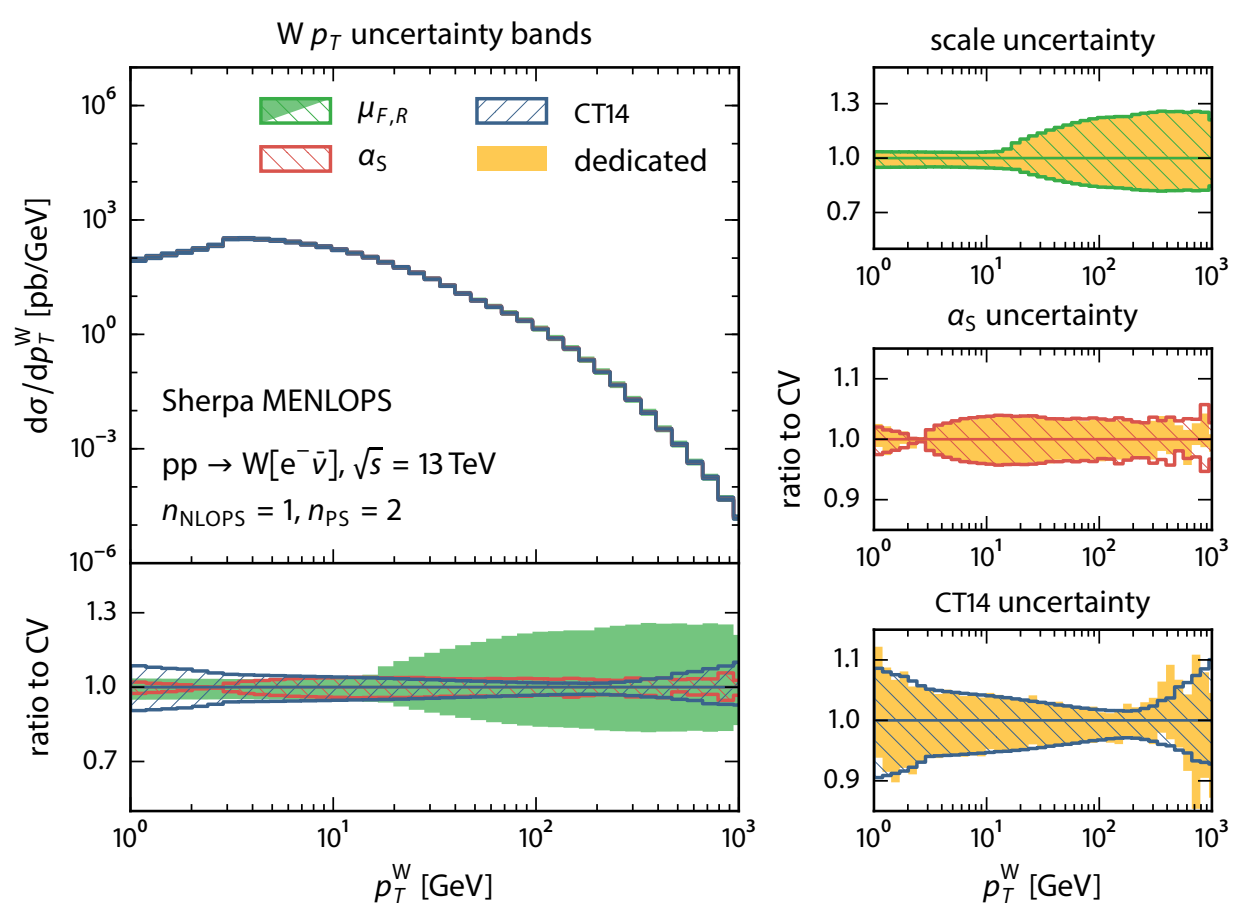

other maximum numbers of reweighted emissions $n_{\text {NLOPS, }} n_{\text {PS }}$

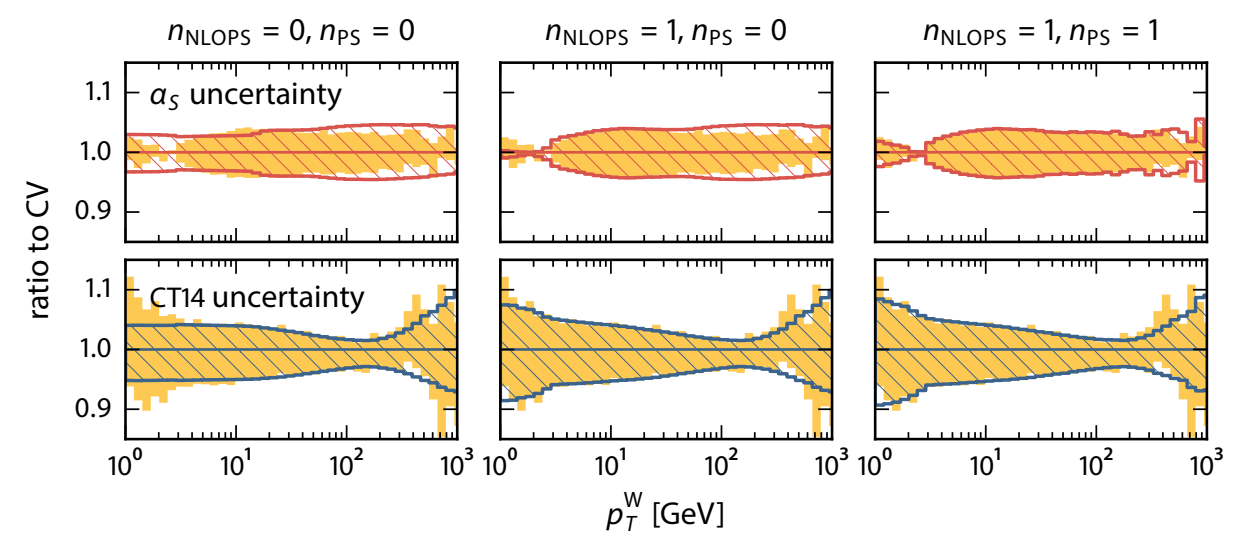

Figure 3.12.: The same as in Fig. 3.11, but for a multi-jet merged generation with one NLO matrix element for the 0-jet multiplicity, and LO matrix elements for the 1- and 2-jet multiplicities. The uncertainty bands are calculated by reweighting the matrix element and a maximum number of emissions from the MC@NLO ( $\left.n_{\text {NLOPS }}\right)$ and the ordinary PS $\left(n_{\mathrm{PS}}\right)$. In the upper four plots, $n_{\text {NLOPS }}=1$ and $n_{\text {PS }}=2$, thus up to three emissions are reweighted. In the lower plots, both $n$ are varied for comparison. Again, we find a saturation when reproducing dedicated calculations for $n_{\text {NLOPS }}+n_{\mathrm{PS}} \geq 2$, with no further improvement when $n_{\mathrm{PS}}$ is increased from 1 to 2 . 

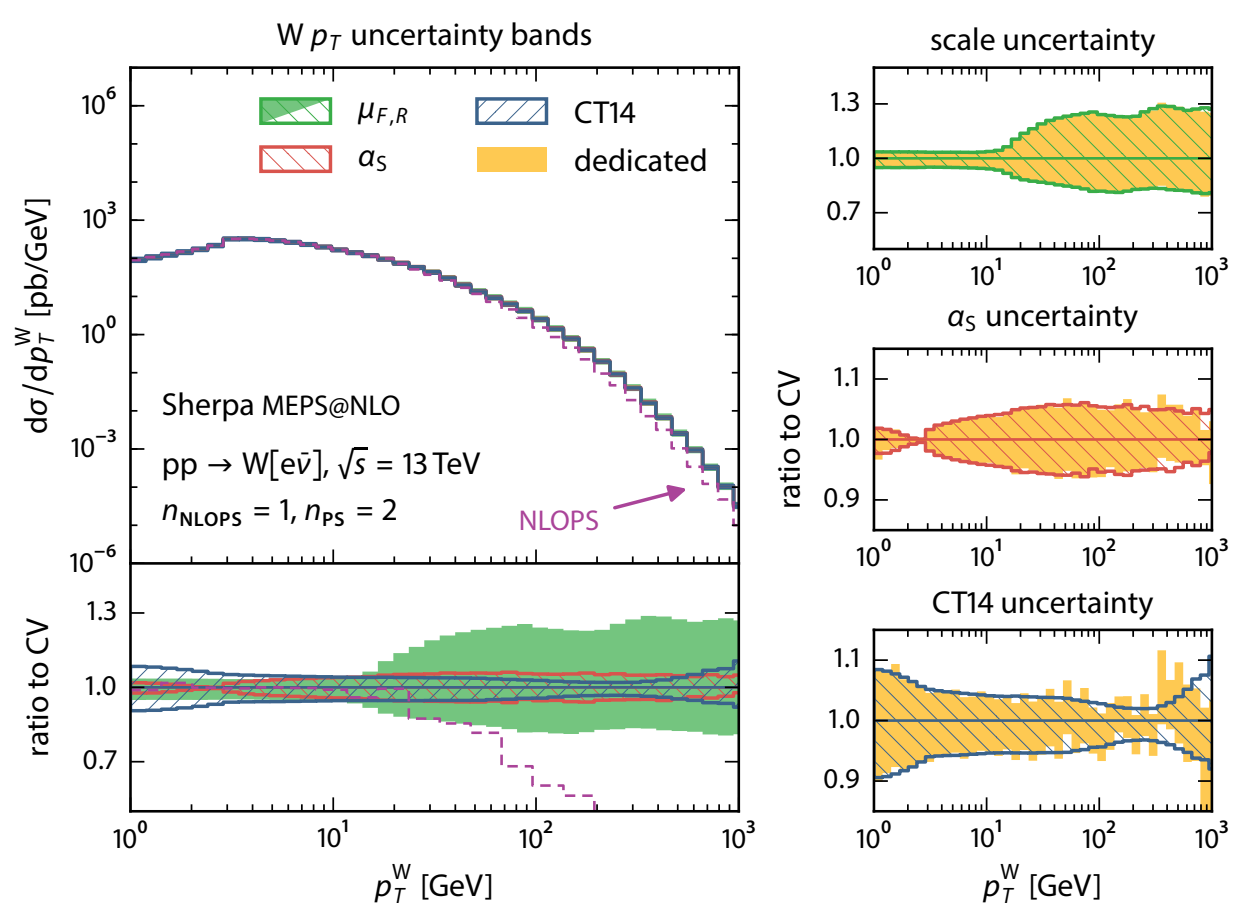

other maximum numbers of reweighted emissions $n_{\text {NLOPS }} n_{\text {PS }}$

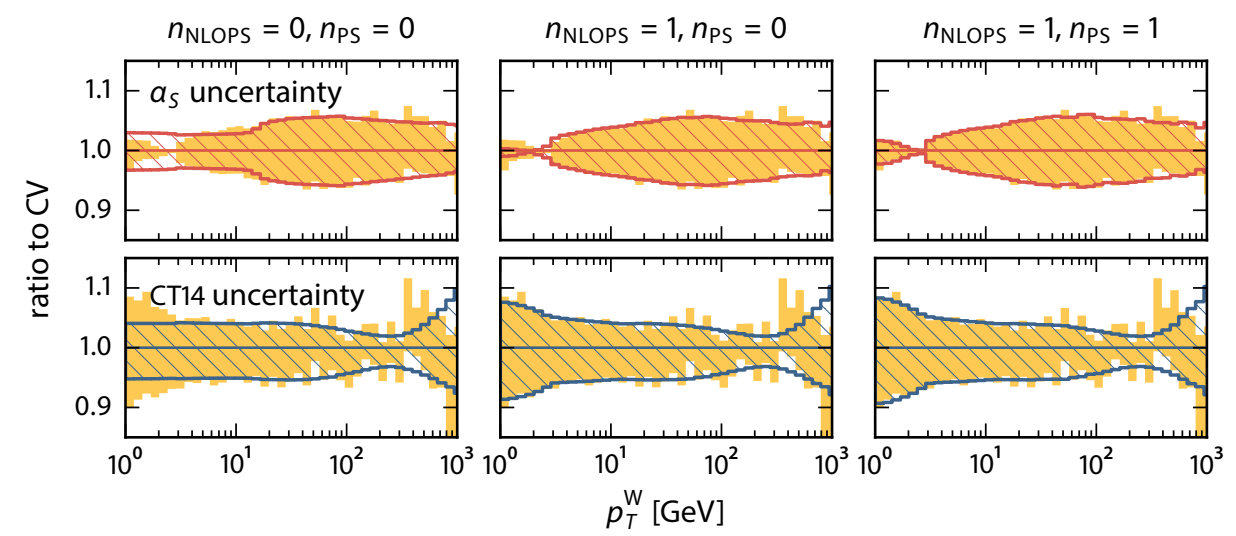

Figure 3.13.: The same as in Fig. 3.12, but for a multi-jet merged generation with NLO matrix elements for the 0 - and 1-jet multiplicities. The uncertainty bands are calculated by reweighting the matrix element and a maximum number of emissions from the MC@NLO ( $\left.n_{\text {NLOPS }}\right)$ and the ordinary PS $\left(n_{\mathrm{PS}}\right)$. In the upper four plots, $n_{\mathrm{NLOPS}}=1$ and $=n_{\mathrm{PS}}=2$, thus up to three emissions are reweighted. In the lower plots, both $n$ are varied for comparison. Again, we find a saturation when reproducing dedicated calculations for $n_{\mathrm{NLOPS}}+n_{\mathrm{PS}} \geq 2$, with no further improvement when $n_{\mathrm{PS}}$ is increased from 1 to 2 . 


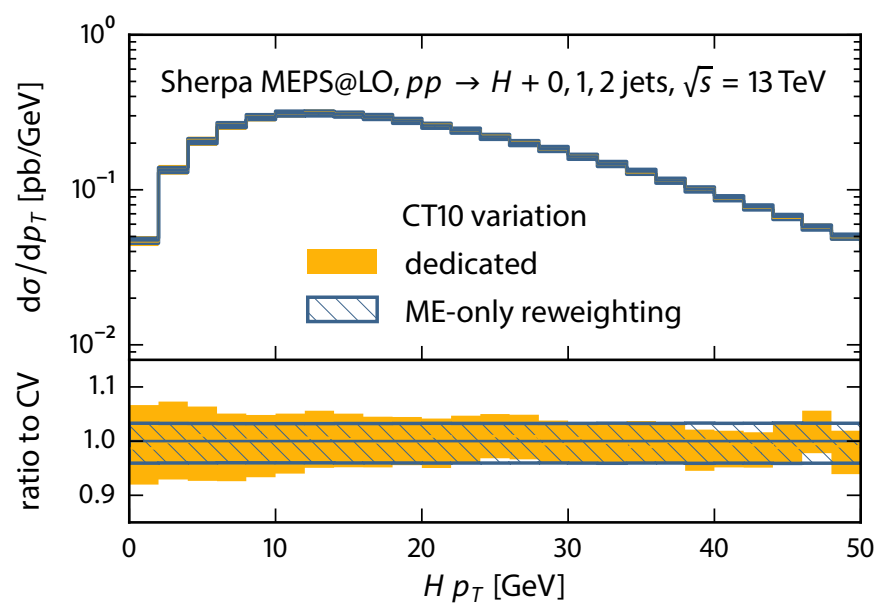

Figure 3.14.: The transverse momentum of the $\mathrm{H}$-boson in $\mathrm{H}$ production. A reweighting is performed to generate the $\mathrm{CT} 10$ uncertainty band, which is compared to a dedicated calculation. The reweighting is restricted to the matrix elements of the Sherpa multi-jet calculation, merging the 0 -, 1- and 2-jet multiplicities and a parton shower.

comparison. Below $Q_{\text {cut }}=20 \mathrm{GeV}$, it is identical to the MEPS@NLO result, but it falls more steeply thereafter, as it describes o jets to NLO and 1 jet to LO, whereas our MEPS@NLO calculation describes the o- and 1-jet multiplicities to NLO, and 2 jets to LO accuracy, such that large $\mathrm{W}$-boson recoils can be predicted with higher (and more faithful) rates.

In all multi-jet merging validations, we find a similar behaviour with respect to the imprint of including emissions in the reweighting. For $n_{\mathrm{NLOPS}}+n_{\mathrm{PS}}=2$, the dedicated calculations are well reproduced, and no further improvement is found for $n_{\mathrm{NLOPS}}+n_{\mathrm{PS}}=3$. It is noteworthy, that for the MENLOPS case we find a worse reproduction for $n_{\mathrm{NLOPS}}=1$ and $n_{\mathrm{PS}}=0$ compared to the NLOPS and the MEPS@NLO cases. This originates in the fact that in the latter two cases, we enable the reweighting of emissions off $\mathbb{S}$-events at all involved multiplicities, whereas in the MENLOPS case only the first of the three multiplicities is affected, because the other two are at LO and therefore do not have $\mathbb{S}$-events. Thus, the overall importance of the $\mathbb{S}$ emission reweighting gets restricted to the region below $Q_{\text {cut }}$ of the 1-jet configuration in the MENLOPS case.

We close this section by noting that the too small uncertainty for very low $p_{T}^{\mathrm{W}}$ for variations of the matrix elements only seems to be a general feature, with the form being independent of the partonic initial state. In Fig. 3.14, we perform a CT1o PDF band closure test for the transverse momentum distribution of the $\mathrm{H}$-boson in $\mathrm{H}$ production. The collider set-up is as for the $\mathrm{W}$ production, i.e. pp collisions at $13 \mathrm{TeV}$. LO matrix elements for $\mathrm{H}$ plus 0,1 or 2 jets are merged. The Higgs is produced via an effective coupling to two incoming gluons via a top-quark loop which is integrated out in the infinite top-mass limit. Hence, our lowest-order initial state is now two gluons instead of a weak quark doublet. However, 
the deviation of the matrix-element-only reweighting band from the dedicated one for low transverse momentum is very similar to what we observed in the $\mathrm{W}$-boson case, although less pronounced.

\subsection{Adding reweighted emissions vs. merging more multiplicities}

To supplement our closure tests for the transverse momentum of the $\mathrm{W}$-boson, and to highlight the differences between a fixed-order plus parton-shower calculation to a multijet merged one, we now consider the exclusive jet multiplicity $N_{\text {Jets. }}$. The process still is W production, at a $13 \mathrm{TeV}$ pp collider, as in the previous section.

The $N_{\text {Jets }}$ observable is not only sensitive to the generation of hard jets (as is the $p_{T}^{\mathrm{W}}$ distribution through their combined recoil), but to the generation of jets in general. As the number of jets itself is binned, possible deviations of the reweighting will be separated by multiplicity. This also allows us to explore the effect of replacing parton-shower emissions with matrix-element calculations, by comparing a LOPS calculation to a MEPS@LO calculation. It is expected that this has a strong impact on higher jet multiplicities, as the parton shower alone can not give a faithful prediction for several emissions that are hard enough to seed new jets.

In Figs. 3.15 and 3.16, we present the results for LOPS and MEPS@LO, respectively, in a similar way as we have done in the $p_{T}^{\mathrm{W}}$ case, i.e. we give nominal results and uncertainty bands according to Table 2.1, along with the ratios to the central value. In addition, we show for comparison the nominal prediction of the MEPS@LO prediction along with the LOPS bands, and vice versa. The uncertainty bands are now generated by a complete reweighting, i.e. of the matrix element(s) and all parton-shower emissions $\left(n_{\mathrm{PS}}=\infty\right)$. Closure tests for the bands against dedicated predictions are also provided. For the $\alpha_{S}$ band, it is repeated for varying numbers of reweighted emissions, $n_{\mathrm{PS}}=0,1,2,3,6$ and 12 . We did not observe significant deviations for the scale and PDF uncertainty when $n_{\mathrm{PS}}$ is varied, and hence these variations are omitted from the figure.

First we note that the nominal MEPS@LO calculation gives increasingly higher cross sections for larger $N_{\text {Jets }}$ bins than the LOPS ones. In fact, both results are only compatible within their respective uncertainties for $N_{\text {Jets }}=0,1{ }^{10}$ This is expected, because in the LOPS calculation, jet multiplicities beyond zero are exclusively filled by parton-shower emissions, which underestimates the probability of jet-seeding emissions. These bins also inherit the (factorisation) scale uncertainty from the single o-jet matrix element. For the MEPS@LO calculation, also renormalisation scale uncertainties are present through the $\alpha_{S}$ factor, and we observe a larger scale uncertainty for larger jet multiplicities, corresponding to the increasing order in $\alpha_{S}$.

Comparing the LOPS results for the $n_{\mathrm{PS}}$ variation of the $\alpha_{S}$ uncertainty band to the MEPS@LO ones, one notes that the variation plays a larger role in the LOPS case, where the

\footnotetext{
${ }^{10}$ In general, this depends on the prescription on how to combine the individual uncertainty bands. However, in this case already the scale uncertainty bands alone overlap.
} 
uncertainty is increasingly over-estimated for larger $n_{\mathrm{PS}}$. In fact, for each increment in $n_{\mathrm{PS}}$, the $\alpha_{S}$ uncertainty of the associated $N_{\text {Jets }}$ bin exceeds beyond the reference band. Partly, this effect also extends to larger $N_{\text {Jets }}$ bins. This is clearly an artificial effect, and we see in the main $\alpha_{S}$ uncertainty plots, that the full reweighting $\left(n_{\mathrm{PS}}=\infty\right)$ performs well. A finite choice of $n_{\mathrm{PS}}$ breaks the unitarity of the reweighted shower, and a discrete binning in exclusive jet multiplicities is expected to be affected most. This is because we do not reweight the rejected trial emissions for the Sudakov form factor between the scale of the last emission (i.e. the $N_{\text {Jets }}$ th emission) and the jet cut parameter, a region of the Sudakov form factor integral that is still relevant to the number of jets. These rejections would counter-act the reweighting of the accepted trial emissions, cf. the third line in Eq. (3.25). For $N_{\text {Jets }} \leq 2$, we see a significant reduction of the over-estimated uncertainties only for our $n_{\mathrm{PS}}=12$ sample. Contrast this to the $\mathrm{W} p_{T}$, where we reweight the relevant (recoil-generating) part of the Sudakov form factor as soon as $n_{\mathrm{PS}} \approx 2$.

For MEPS@LO, the uncertainty growth is only visible for the $N_{\text {Jets }}=3$ bin, when we move from $n_{\mathrm{PS}}=0$ to 1 . Considering the band shapes, we observe that the $n_{\mathrm{PS}}=0,1$ ones in the MEPS@NLO case resemble the $n_{\mathrm{PS}}=2,3$ band shapes in the LOPS case. This supports the conclusion, that the $\mathrm{PDF} / \alpha_{S}$ reweighting behaves similarly, when $n_{\mathrm{PS}}$ plus the highest number of jets described by a matrix element, $n_{\mathrm{ME}}$, are equal, since $n_{\mathrm{ME}}=2$ for our MEPS@NLO calculation. This likely is an observable-specific statement. Any $N_{\text {Jets }}$ bin will be dominated by a matrix element of the same multiplicity, if available. This in turn will lead to a reweighting of a sequence of backward-clustered splittings of the same length, which has the same dependence structure as an equal number of accepted parton-shower trial emissions, cf. Eqs. (3.33) and (3.43). This clear picture should be diluted in observables that are less descriminating with respect to the number of jets.

\subsection{Using over-estimate factors for a smoother parton-shower reweighting}

In Section 3.3.3 we discussed the possibility to shift the over-estimated parton-shower kernel $\hat{\mathrm{K}}$ by a constant factor $k_{\hat{\mathrm{K}}}$ to make sure that the denominator of the rejected trial-emission reweighting factor, $(\hat{\mathrm{K}}-\tilde{\mathrm{K}}) /(\hat{\mathrm{K}}-\mathrm{K})$, given in Eq. (3.25), stays away from zero. Choosing $k_{\hat{\mathrm{K}}}$ involves compromising between the efficiency of the parton shower and the numerical stability of its reweighting. For the observables and processes (boson production) studied so far, the reweighting procedure appeared stable, at least when we enforced a cut-off on the deviation of the reweighting factors $\mathcal{Q}$ from unity, cf. Section 3.3.3.

To complement this, we now look at another process/observable pair, which is the dijet azimuthal decorrelation in $\mathrm{pp} \rightarrow \mathrm{jj}$ events at $7 \mathrm{TeV}$, where the jets are defined with the anti- $k_{T}$ algorithm with a radius parameter $R=0.6$. The two hardest jets are required to be central, with a rapidity $|y|<0.8$ and a minimum jet $p_{T}$ of $100 \mathrm{GeV}$. Compared to dijet events at a leptonic collider and $\mathrm{W}$ production at a hadronic collider, this is the first time we look at a process which comprises all possible initial-state partonic channels already 

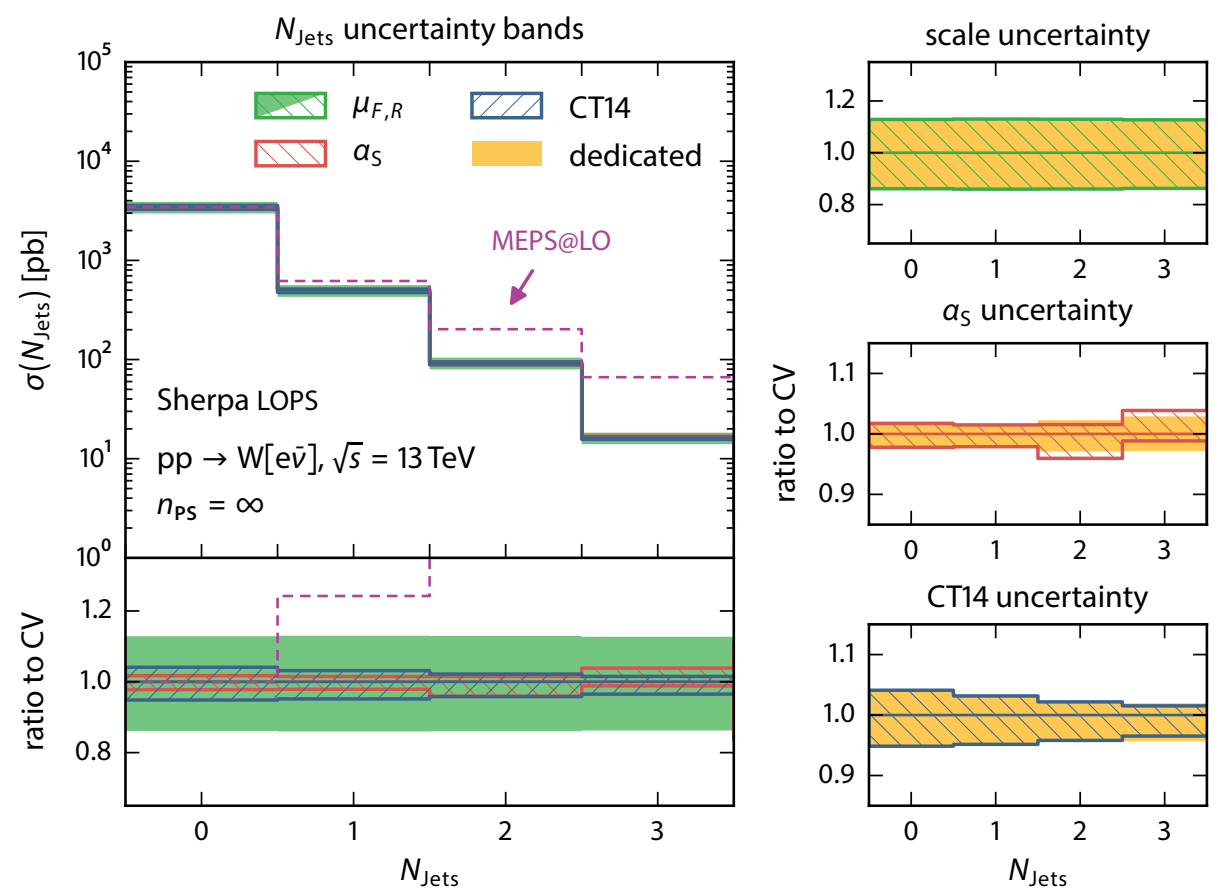

other maximum numbers of reweighted emissions $n_{\mathrm{PS}}$ for the $a_{S}$ uncertainty

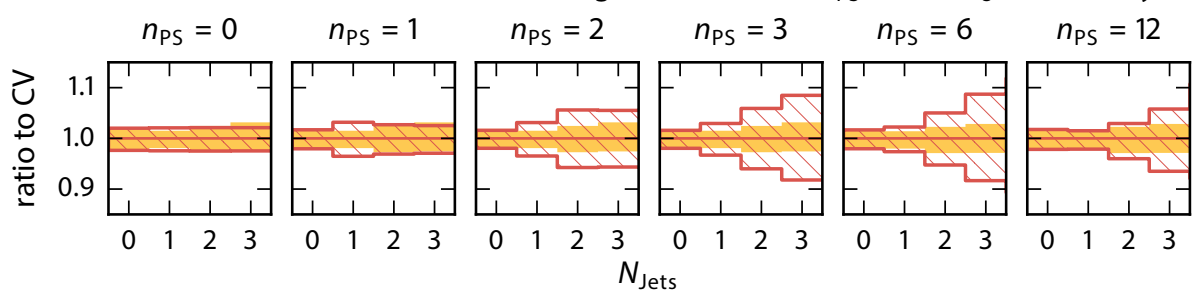

Figure 3.15.: The exclusive number of jets $N_{\text {Jets }}$ for LOPS W-boson production, with uncertainty bands according to Table 2.1. For comparison, the nominal prediction of a MEPS@LO calculation presented in Fig. 3.16 is shown. 

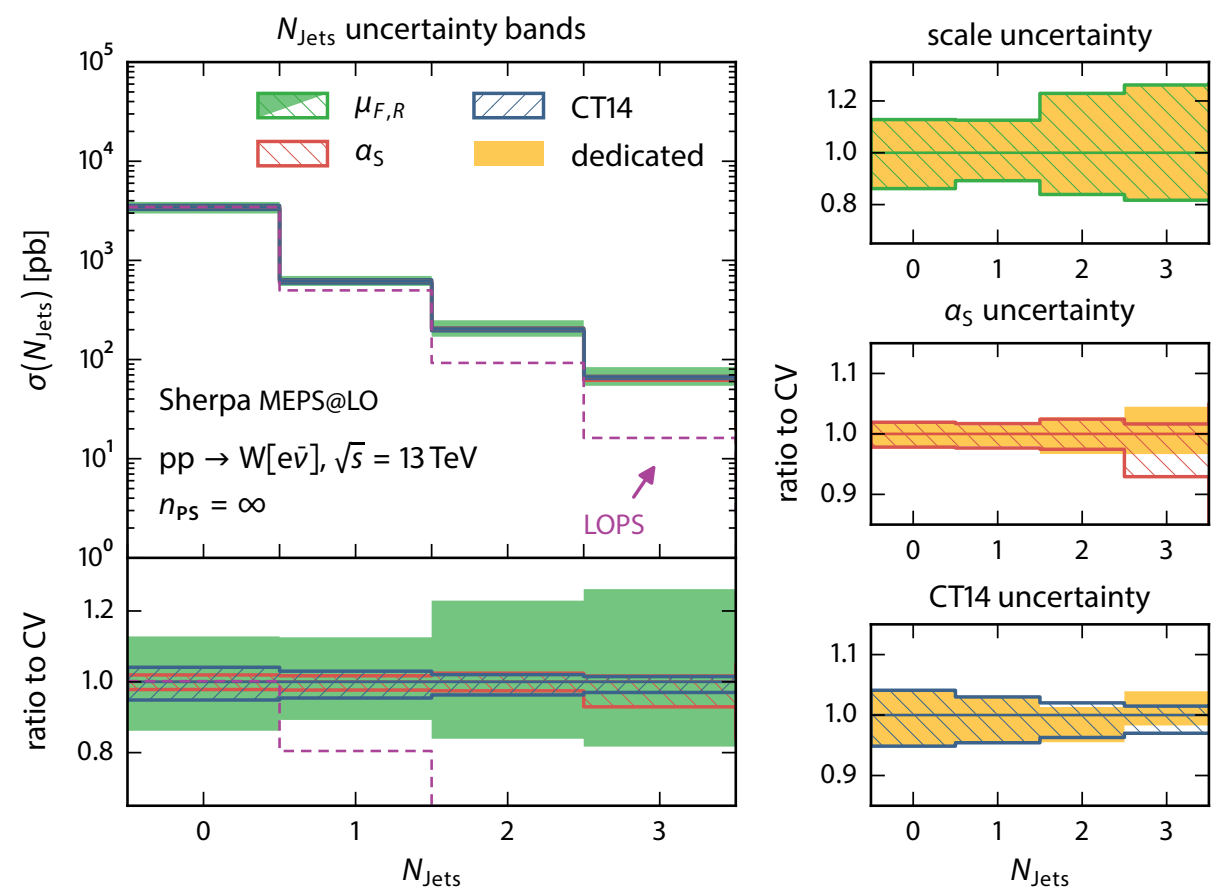

other maximum numbers of reweighted emissions $n_{\mathrm{PS}}$ for the $a_{S}$ uncertainty

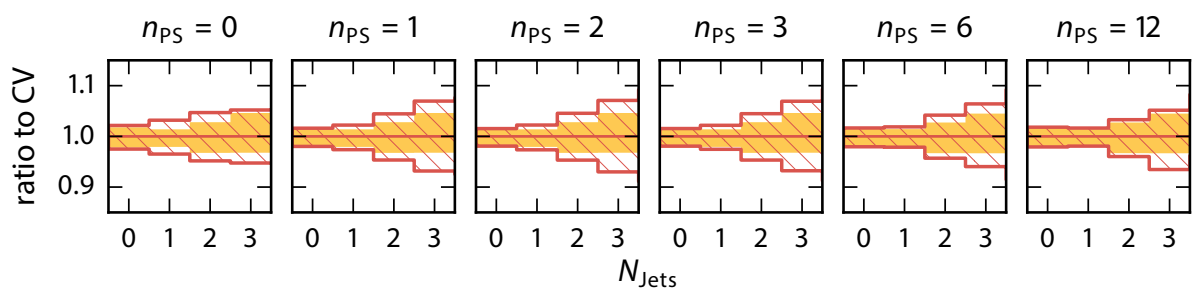

Figure 3.16.: The same as in Fig. 3.15, but for a MEPS@LO calculation with matrix elements for the 0-, 1- and 2-jet multiplicities. For comparison, the nominal LOPS result presented in Fig. 3.15 is reproduced. 
at LO, including channels with one or two gluons. We have observed in Section 3.3.3, that splittings behave differently with respect to the distribution of reweighting factors, and hence the consideration of this gluon-induced process is complementary to our previous discussions. The dijet azimuthal decorrelation is the azimuthal angle $\Delta \phi$ between the two jets with the largest $p_{T}$. At lowest order, we always have a back-to-back configuration, i.e. the maximum value $\Delta \phi=\pi$, due to momentum conservation. If we add higher-order corrections, the momentum is potentially shared with additionally resolved jets, leading to smaller $\Delta \phi$. For $\Delta \phi \rightarrow \pi$, hard emissions off the two leading jets are suppressed, and the behaviour of the soft emissions determine the amount of smearing away from $\Delta \phi=\pi$. To produce configurations with low values of $\Delta \phi$, one or more hard emissions are necessary. In fact, each multiplicity has a minimum value of $\Delta \phi$, corresponding to the case, where the transverse momenta of all jets are equal. It follows that the azimuthal angles between them are all the same, $2 \pi / N_{\text {jets }}$. Hence, we have staggered regions towards $\Delta \phi=0$, with each region being dominated by events with a given number of hard emissions. Naturally, the parton shower gives the best prediction in the $\Delta \phi \rightarrow \pi$ region, whereas fixed-order corrections are necessary to describe the lower- $\Delta \phi$ region.

In Fig. 3.17, we show the nominal prediction of a SHERPA LOPS calculation, associated with uncertainty bands as defined in Table 2.1 generated with the on-the-fly reweighting including the matrix-element and all parton-shower emissions $\left(n_{\mathrm{PS}}=\infty\right)$. The calculation is binned additionally in the hardest jet transverse momentum, here we show only the region with $110<p_{T}^{\max } / \mathrm{GeV}<160$. The scales are set to $\mu_{R}=\mu_{F}=H_{T}$, i.e. to the scalar sum of the transverse momenta of all jets,

$$
H_{T}^{2}=\left(\sum_{i} p_{T, i}\right)^{2} .
$$

The statistics is deliberately kept small, as we intend to study numerical instabilities with respect to a possible choice of $k_{\hat{\mathrm{K}}}=3$. However, the scale and PDF uncertainty bands are smooth and feature the expected growth towards small values of $\Delta \Phi$. Note that the scale uncertainty is artificially small at $\mathrm{LO}$ for small and very large $\Delta \Phi$ bins. This is because these bins are dominated by many emissions, and those are not part of the LO matrix-element calculation. Note also that the bands feature a constriction below the first bin. This is a consequence of the dominating statistics of the first bin and the normalisation of the distribution to the total cross section.

If we consider now the $\alpha_{S}$ uncertainty band, we observe that this choice of $k_{\hat{\mathrm{K}}}=3$ still leads to fluctuations at low values of $\Delta \Phi$ (remember that the $\alpha_{S}$ reweighting also entails PDF reweightings, with ratios that are usually larger compared to a pure PDF uncertainty band). However, we contrast the uncertainty bands for $k_{\hat{\mathrm{K}}}=3$ with ones that are generated from a reweighting with $k_{\hat{\mathrm{K}}}=1$ on the right-hand side of Fig. 3.17. The latter fluctuate strongly even for intermediate values $\Delta \Phi \approx 0.8$, and to a lesser degree also for $\Delta \Phi \approx 1.0$, i.e. in the regions with the largest statistics. We conclude that an over-estimate factor $k_{\hat{\mathrm{K}}}>1$ improves the statistical behaviour of the $\alpha_{S}$ reweighting. Given more experience, an appropriate default 

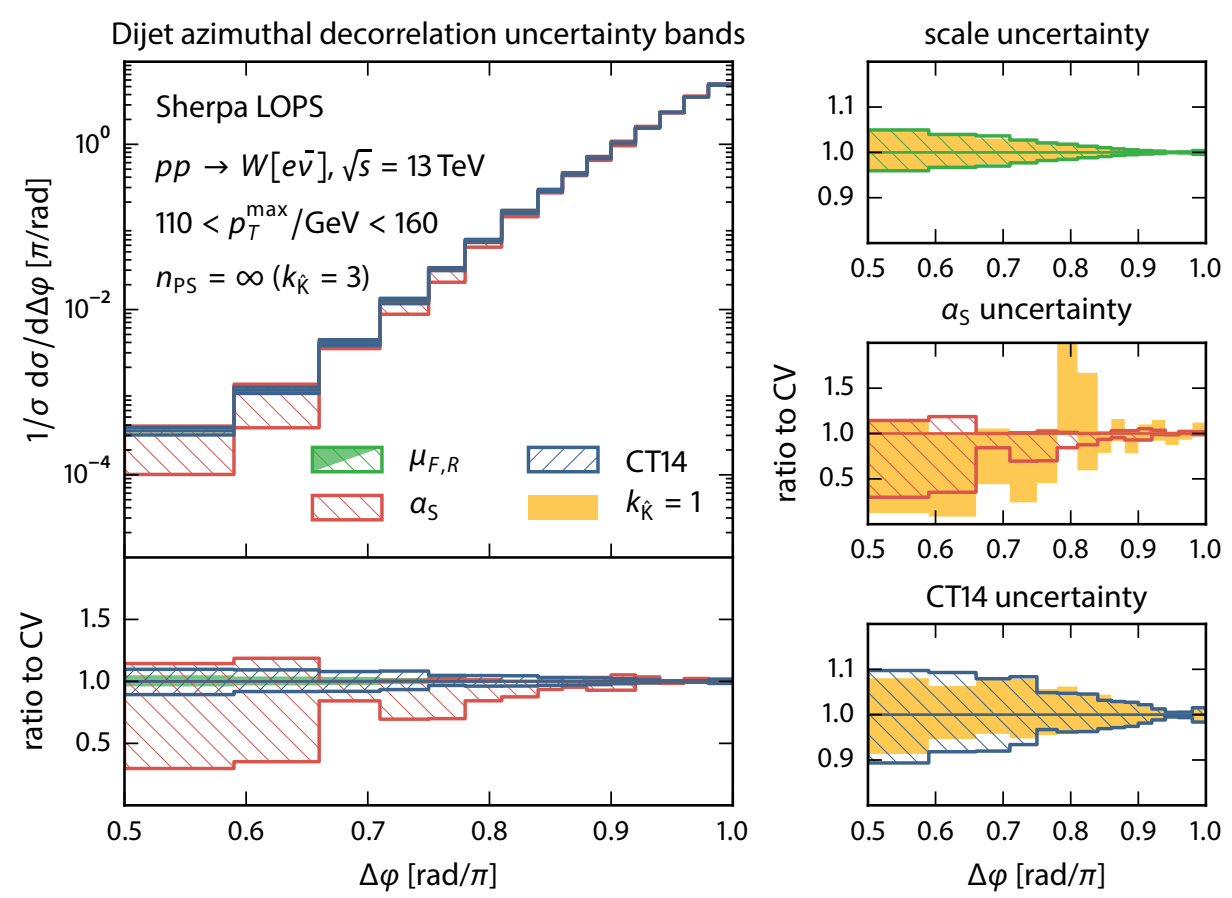

Figure 3.17.: The dijet azimuthal decorrelation for LO plus parton-shower dijet production, with $p_{T}^{\max }$ between 110 and $160 \mathrm{GeV}$. The uncertainty bands are defined according to Table 2.1 and generated with an on-the-fly reweighting of the matrix-element and all parton-shower emissions. The over-estimate parton-shower kernel $\hat{K}$ is multiplied by a factor of $k_{\hat{K}}=3$ in the left-hand side panel. In the panels on the right-hand side, the individual uncertainty bands are compared to a reweighting with $k_{\hat{\mathrm{K}}}=1$.

value should be considered.

\subsection{Preserving the formal accuracy in variations}

In general, the renormalisation and factorisation scales, $\alpha_{S}$ and the PDFs should be varied consistently throughout any of the presented calculations. While at fixed order the situation is clear, the matched and merged approaches allow for some degree of freedom regarding partial variations while still retaining their respective accuracies.

In the simplest case, LOPS, $\mu_{R}$ and $\mu_{F}$ of the short distance cross section and the parton shower may be varied independently as these variations can be expressed as higher-order terms in a perturbative expansion in the coupling parameter $\alpha_{S}$. This is not the case for $\alpha_{S}$ itself and the PDFs as they are fixed through measured input values and parametrisations. Changes in these input values cannot be expressed as higher-order terms. Thus they need to be chosen consistently throughout the matrix element and the parton shower. 
Similarly, in NLOPS calculations, $\mu_{R}$ and $\mu_{F}$ may be varied in the matrix element ( $\overline{\mathrm{B}}$ and $\mathrm{H}_{A}$ ) or the parton shower (PS $\mathrm{NLOPS}_{\text {and }}$ PS) separately, losing neither the fixed-order nor the resummation accuracy. As the pseudo-subtraction through the $\mathrm{D}_{A}$ in any case employs different scales in $\mathrm{PS}_{\mathrm{NLOPS}}$ and the $\overline{\mathrm{B}}$ and $\mathrm{H}_{A}$ functions, it always leaves remainders of $\mathcal{O}\left(\alpha_{S}^{2}\right)$. Hence, further scale variations in either one, the short-distance cross sections or the $\mathrm{PS}_{\mathrm{NLOPS}}$, do not worsen the nominal accuracy of the method. Retaining the logarithmic accuracy of the parton shower on the other hand requires identical renormalisation and factorisation scales throughout all resummation-relevant components, i.e. $\mathrm{PS}_{\mathrm{NLOPS}}$ and PS. Again, variations in $\alpha_{S}$ or the PDFs need to be consistent throughout the calculation.

The multi-jet merged calculations impose further constraints since they treat multijet matrix elements and parton-shower emissions on the same footing. The notation of the scales already reflects this for $\mu_{R}$ and $\mu_{F}$. In their definitions only the core scales remain as free parameters and may be varied independently. Again, the $\alpha_{S}$ and PDF parametrisations need to be the same throughout.

\subsection{CPU time measurements}

The benefit of reweighted calculations is given by the saving of CPU time. In order to evaluate the gain, we shall compare the event generation time of reweighted calculations with the sum of generation times for all corresponding dedicated computations. Here we consider both parton-level calculations, as well as runs including multiple interactions and a fragmentation model, the typical default in physics analyses applications. For the latter it can be expected that the gain in CPU time by using the reweighting approach is most considerable, as the CPU-intense non-perturbative event generation phases do not need to be re-evaluated. In what follows, we compare actual event-generation times, neglecting the set-up times of the individual runs. ${ }^{11}$

In Fig. 3.18, we consider event generations using LOPS, NLOPS, MEPS@LO and MEPS@NLO calculations for $\mathrm{pp} \rightarrow \mathrm{W}\left[\mathrm{e}^{-} \bar{v}\right]$ at $13 \mathrm{TeV}$. The ratio of $\mathrm{CPU}$ time between the reweighting and dedicated generations is shown for different maximum numbers of reweighted partonshower emissions $n_{\mathrm{PS}}+n_{\mathrm{NLOPS}}$. Whether non-perturbative effects are included or not, the time needed for the reweighting calculation is below $10 \%$ of the time needed for dedicated calculations if only the matrix element is reweighted $\left(n_{\mathrm{PS}}=n_{\mathrm{NLOPS}}=0\right)$. The ratio then increases with a larger number of reweighted emissions, as their reweighting needs additional time, asymptotically approaching the value when all parton-shower emissions are reweighted. For parton-level-only calculations, this ratio is around 0.35 for LOPS events, and around 0.3 for NLOPS events. This reduction can be explained due to relatively smaller computational cost of the parton shower as a whole when the rest of the calculation is

\footnotetext{
${ }^{11}$ If NLO matrix elements at higher multiplicities are needed for an event generation, the time needed for the integrator optimisation and the process selection weight optimisation can be quite substantial, e.g. a couple of days. In the case of unweighted event generation, this even has to be re-done for every single parameter variation, as the channel weights are used for the unweighting. When reweighting is used, this is not necessary and so even more CPU is saved.
} 

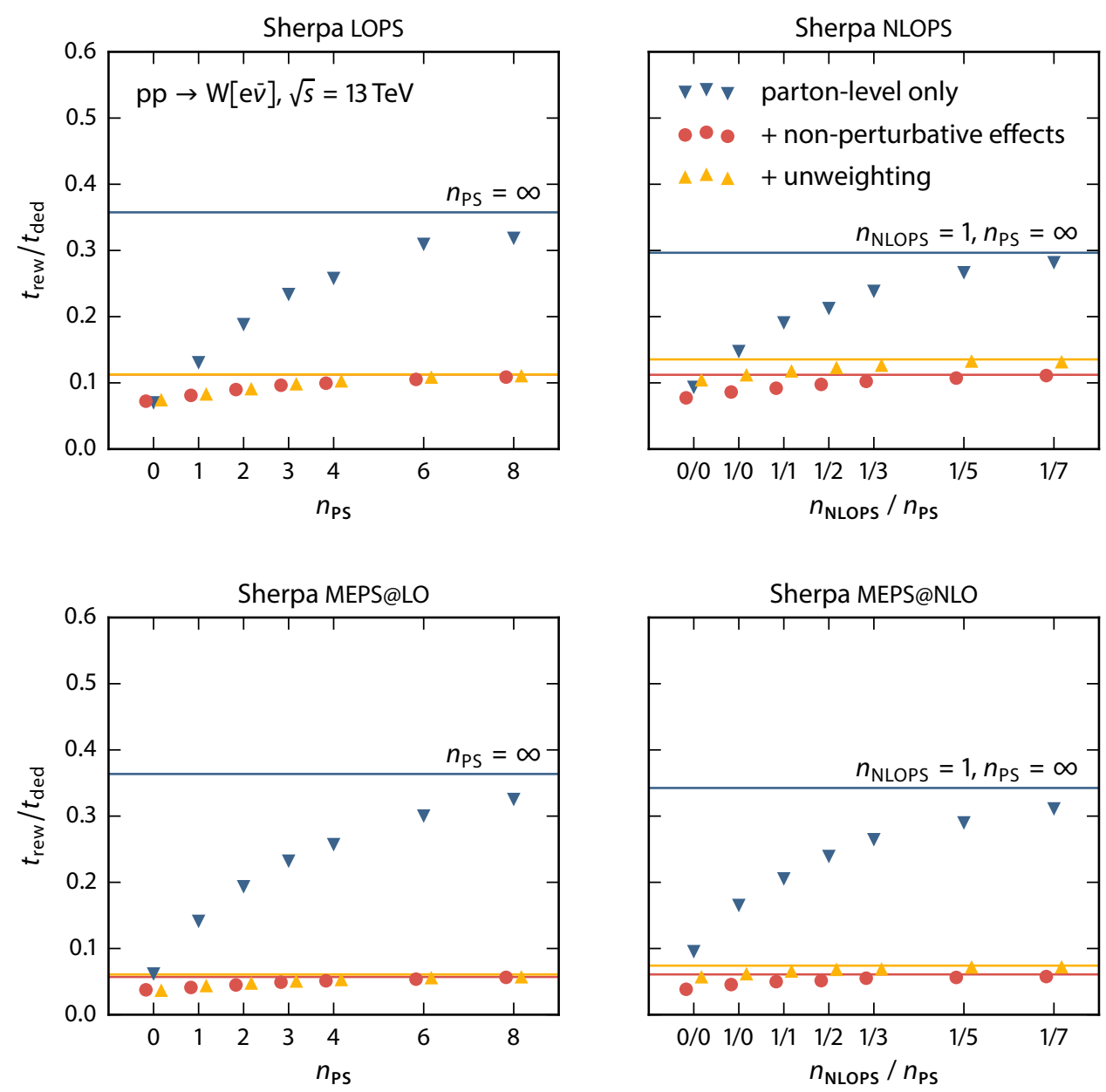

Figure 3.18.: The ratio of CPU time needed for a reweighting calculation $t_{\text {rew }}$ over the time needed for generating predictions for all variations with dedicated runs $t_{\text {ded }}$. The reweighting includes up to $n_{\text {PS }}$ parton-shower emissions. The sampled variations are listed in Table 2.1. Parton-level-only results are compared to results for calculations including multiple interactions and hadronisation effects ("+ non-perturbative"), and to calculations where in addition to adding non-perturbative effects the events have also been unweighted ("+ unweighting"). The ratios for reweighting all emissions are indicated with a horizontal line.

more complex. Also note that $n_{\mathrm{PS}}$ for LOPS is only equivalent to $n_{\mathrm{PS}}+n_{\mathrm{NLOPS}}$ for $\mathbb{S}$ events. $\mathrm{H}$ events do not feature the S-MC@NLO-emission, and hence for them $n_{\mathrm{NLOPS}}$ does not contribute to their reweighting.

For the same reason, when non-perturbative effects are included, that ratio drops to about o.1: The parton shower (and its reweighting) plays a smaller role in terms of CPU cycles, when multiple interactions and hadronisation is enabled.

If on top of the non-perturbative effects the events are also unweighted, the ratio does 
not change in the LOPS case, but in the NLOPS case (by about $20 \%$ ). A reason might be, that only in the NLOPS a sizeable number of events gets rejected. For these, the jet evolution and non-perturbative phases are not performed at all, whereas the matrix-element calculation (and its reweighting) is always done, for accepted and rejected events alike. The same is true for the S-MC@NLO emission from S events. As a consequence, the relative cost of the reweighting grows slightly. A future improvement of the implementation would postpone these reweightings to a time point after the possible rejection. This of course requires that the dependence of the rejection probability is negligible. For the observables studied so far this was found to be true, at least to $\mathcal{O}\left(10^{-4}\right)$. Another speed-up will be provided as soon as the Rivet framework and the HepMC event records will have a genuine support for event weight variations, which is expected for the upcoming major releases. Currently the RivET analysis is repeated for every variation. This is not necessary, because the reweighting only change the overall weight, but does not modify the final state (or its kinematics). Only the filling itself and the histogram storage must be duplicated for each variation, which is much less time-consuming than the analysis itself.

Note that the effective gains will be lower than the results presented in this section, when we take into account the reduced statistical accuracy after the parton-shower reweighting. This requires more events to be generated in a reweighting calculation to reach the same statistical accuracy as in a dedicated calculation.

\subsection{Discussion}

In this chapter we have discussed a new reweighting method for perturbative QCD and validated it with closure tests.

The approach is an on-the-fly reweighting and is implemented within the SHERPA event generation framework, and will be part of its next release. It is connected to all parts of the event generation loop that are related to the calculation of the event weight (or its unweighting), as is schematically depicted in Fig. 3.19. It supports weighted and unweighted event generations. All fully automated state-of-the-art calculational methods for perturbative QCD are supported, i.e. fixed-order (LO \& NLO), fixed-order plus parton shower (LOPS \& NLOPS), and multi-jet merged calculations (MEPS@LO, MEPS@NLO and the mixed case, MENLOPS). Of these, only the reweighting of LO and NLO calculations (and to some extent LOPS) was available before. The semi-automated NNLO (+ parton-shower) calculations are not yet supported. However, the recent implementation of NNLO + parton-shower calculations via the $\mathrm{UN}^{2}$ LOPS scheme in SHERPA make this a natural next step for future developments, by extending the methods presented in this chapter. The increased CPU time consumption of NNLO calculations will even strengthen the motivation for an efficient reweighting, and an on-the-fly approach would not suffer the storage issues of an a-posteriori approach with respect to NNLO [128, 138].

Our implementation allows for both scale and parameter variations. Scale variations affect either the ME-only $\mu_{R}$ and $\mu_{F}$, or also the input scales of PDF and $\alpha_{S}$ of parton- 


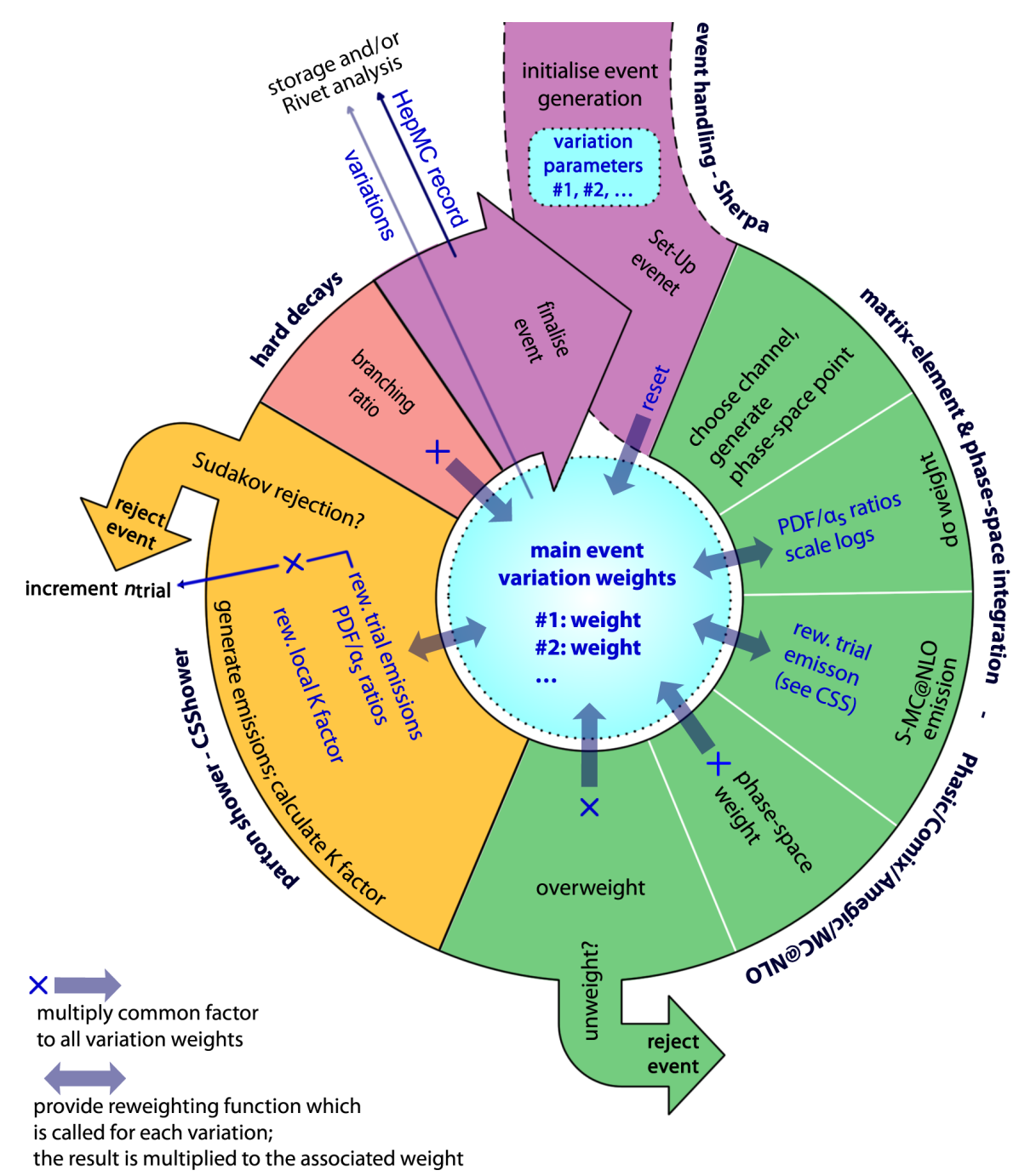

Figure 3.19.: A simplified diagram of the internal reweighting implementation (blue) within the Sherpa event loop parts that are relevant to the event weight calculation. Event phases (and the associated Sherpa modules) are indicated outside the circle. 
shower emissions, including those that are pre-set by the clustering in a multi-jet merged calculation. The scales can be varied independently with prefactors, such that individual scale uncertainties or combined uncertainties, like from a 7-point scale variation, can be studied. The parameters that can be varied are the PDFs, and the value of $\alpha_{S}\left(m_{\mathrm{Z}}^{2}\right)$. These choices are the main parametric sources for QCD uncertainties.

The on-the-fly reweighting does not allow for a variation of the merging scale $Q_{\text {cut }}$, or the parton-shower starting scale $\mu_{Q}$. Also the splitting scales are kept fix, which is however a feature of the reweighted SVA. A parton shower is also defined through its recoil scheme, which can also not be reweighted with our current technology. The form of the splitting kernels $\mathrm{K}$ is also fixed in the current implementation, although in this case only straightforward modifications would be required to change that, since the reweighted SVA allows for an arbitrarily modified $\mathrm{K}$.

We have seen that a parton-level simulation with full on-the-fly variations to 7 scale variations, $56 \mathrm{PDF}$ variations and $5 \alpha_{S}$ variations, is about three times faster than running all variations in separate runs. If only the matrix element is reweighted, the reweighting is faster by about a factor of ten to twenty. The same is true, when non-perturbative effects are enabled in the simulation. Unweighting the events on top of that does not change this gain. However, this is because the current implementation also reweights events that are rejected by the unweighting. In a future update, the reweighting will be postponed to after the unweighting as far as possible, to only reweight accepted events. This is expected to make the reweighting of an unweighted sample much faster. It should also be noted, that running the events through a (usually complex and time-consuming) detector simulation will increase the time gain considerably. Hence, applications involving detector-level data comparisons will profit most by the internal reweighting. Besides the gain in CPU time, the reweighting approach has the additional advantage to reduce the storage requirements of event records significantly, because only the overall event weight is varied, whereas all other event data is not affected by the reweighting and therefore does not need to be duplicated.

We suggest to use scale variations on the matrix-element level as a default, as it is the most important source of uncertainty at LO and NLO, and with the restriction to the fixed-order part of the calculation, the event generation time hardly increases. If the full uncertainty is needed, $\mathrm{PDF} / \alpha_{S}$ variations should be included, and a low-statistics study can tell if and how many parton-shower emissions should be included in the reweighting, to keep the CPU usage of the reweighting small. A rule of thumb is that no parton-shower emission reweighting is necessary for the total cross section (this is ensured by the unitarity of the shower), and 3 emissions are necessary for differential observables, that are affected by the recoil of the hardest shower emissions, but are otherwise inclusive to jet evolution. The reweighting of all emissions is necessary for more exclusive jet observables. Here, a small number of reweighted emissions can actually be worse than a ME-only reweighting, as we have observed for the exclusive jet multiplicity.

The comprehensive reweighting implementation presented in this chapter can greatly speed up the evaluation times for both individual studies and the centralised high-statistics productions of the major experiments at the LHC and future colliders. Other applications, 
where the exact variation is not known beforehand and/or where the associated prediction must be known within milliseconds, call for the use of interpolation grids, which we discuss in the next chapter. 


\section{QCD interpolation grids}

The decomposition of fixed-order QCD calculations into scale/parameter-dependent and -independent bits employed in the last chapter is also a necessary ingredient to produce cross-section grids as provided by the APPLGRID [51] and FASTNLO $[52,53]$ tools. These store the perturbative coefficients for a certain observable calculation discretised in $x$ and $Q^{2}$ using interpolation methods. By this, they get rid of the expensive sum over events that is still necessary in reweighting methods, that operate on single events during or after the event generation. This greatly speeds up a posteriori inclusions of PDFs, $\alpha_{S}$ and scale variations. In turn, such techniques are well suited for (combined) fits of PDFs and $\alpha_{S}$ that require a multitude of re-computations of the theoretical predictions, but can also be used for uncertainty studies.

Until recently, only a limited set of specialised interfaces to NLO codes existed for both APPLGRID and FASTNLO. These separate interfaces did not include a fully general NLO code, and required a large amount of duplication in terms of analysis code, for the necessary projection onto the observable. Over the last years, tools have been developed to fill this gap, by automating the projection of arbitrary NLO QCD calculations onto such grids, namely the AMCFAST [57] and the MCGRID [54-56] packages, which are both used e.g. by the NNPDF collaboration [99]. The first one produces APPLGRIDs with MADGRAPH5_AMC@NLO [163] and MADANALYSIS5 [164], the latter APPLGRIDs or FASTNLO grids from SHERPA events projected on the observables through RIVET [106]. On the "postproduction" side, the APFELGRID [165] tool has been developed. It provides an improved convolution method for use with APPLGRID files that further speeds up the re-evaluations.

In this chapter, we first give a concise review of how to make use of the fixed-order decompositions to fill those interpolation grids in a generic and automated way. After that, we present closure tests for fixed-order interpolation grids generated with MCGRID and, building on these, additional tests for various improvements that have been developed in the context of this thesis within MCGrid and SHERPA. These improvements led to the 2.o release of MCGRID [55]. One of them is the treatment of the $\mathcal{O}\left(\alpha_{S}\right)$ expansion of S-MC@NLO NLOPS calculations, as a first preparation for a future inclusion of all-order parton-shower dependences. Other ones are the support for the dedicated scale logarithm grids within APPLGRID, and the support for filling FASTNLO grids. Lastly, a consistency test is performed, where we show that we can fully reproduce NLOPS uncertainty bands as with the internal reweighting, provided that we omit the reweighting of parton-shower emissions in the latter. We find the same deviations from a dedicated calculation. From the internal reweighting, we know that these deviations can be attributed to the omission of the shower dependences, and hence we conclude this chapter with an outlook on a possible 
extension of interpolation grids to include those.

Some of the contents in this chapter are published in $[55,56]$.

\subsection{Encoding cross sections on interpolation grids}

\subsubsection{Multiplicative $\alpha_{S}$ and PDF dependences}

If we go through the LO and NLO formulae Eqs. (3.2) and (3.5) for calculating an observable $O$ at fixed leading- or next-to-leading order, we can make an observation, which is the basis of the QCD interpolation grid method. We can decompose all contributions into terms that share a common form,

$$
\langle O\rangle^{(\mathrm{N}) \mathrm{LO}}=\sum_{\text {terms }} \int \mathrm{d} \Phi\left(\frac{\alpha_{S}\left(\mu_{R}^{2}\right)}{2 \pi}\right)^{n} f_{a}\left(x_{1}, \mu_{F}^{2}\right) f_{b}\left(x_{2}, \mu_{F}^{2}\right) w_{a b}(\Phi) O(\Phi),
$$

where the exact definitions of the perturbative order in QCD $n$, the phase space $\Phi$, the scales $\mu_{R}$ and $\mu_{F}$, the partons $a / b$ and their momentum fractions $x_{1 / 2}$ depend on the term type. For example, $\Phi$ is either $\Phi_{B}, \Phi_{R}$ or $\Phi_{B} \cdot \Phi_{1}$, and the $x$ possibly contain the partonic momentum fractions $x^{\prime}$ for the KP contribution, see Eq. (3.8). Moreover, $a$ and $b$ do not always label incoming partons, otherwise we would need to keep terms that involve dipole splitting maps separate. These are found in the KP and the $\mathrm{D}_{S}$ contributions. ${ }^{1}$ The weights $w_{a b}$ contain everything else. They do not depend on $\alpha_{S}$ or the PDFs, but if the term is part of a VI or KP contribution, they depend on $\mu_{R}$ and $\mu_{F}$, respectively. From Eqs. (3.7) and (3.8), we can read off how they transform under $\mu_{R} \rightarrow \tilde{\mu}_{R}$ or $\mu_{F} \rightarrow \tilde{\mu}_{F}$ :

$$
\begin{aligned}
& w_{a b}^{\mathrm{VI}} \equiv \mathrm{VI}^{\prime} \rightarrow \mathrm{VI}^{\prime}+c_{R}^{\prime(0)} l_{R}+\frac{1}{2} c_{R}^{\prime(1)} l_{R}^{2} \\
& w_{a b}^{\mathrm{KP}} \equiv \begin{cases}\tilde{c}_{F, a / b}^{(i)} \rightarrow \tilde{c}_{F, a / b}^{(i)}+\bar{c}_{F, a / b}^{(i)} l_{F} & \text { if } i=1,3 \\
x_{a / b}^{\prime} \tilde{c}_{F, a / b}^{(i)} \rightarrow x_{a / b}^{\prime}\left(\tilde{c}_{F, a / b}^{(i)}+\bar{c}_{F, a / b}^{(i)} l_{F}\right) & \text { if } i=2,4,\end{cases}
\end{aligned}
$$

with $l_{F}=\log \left(\tilde{\mu}_{F}^{2} / \mu_{F}^{2}\right)$ and $l_{R}=\log \left(\tilde{\mu}_{R}^{2} / \mu_{R}^{2}\right)$. Otherwise the replacements $\alpha_{S} \rightarrow \tilde{\alpha}_{S}, f \rightarrow \tilde{f}$, $\mu_{R} \rightarrow \tilde{\mu}_{R}$ and/or $\mu_{F} \rightarrow \tilde{\mu}_{F}$ only affect the prefactors to $w_{a b}$ in Eq. (4.1), and can therefore be applied by multiplying with the appropriate $\alpha_{S}$ and PDF ratios.

In a Monte-Carlo integration, we replace the phase-space integral with a sum over phasespace points and only sum over the terms that are present in a given event $e$ :

$$
\langle O\rangle^{(\mathrm{N}) \mathrm{LO}}=\sum_{e} \sum_{\text {terms in } e}\left(\frac{\alpha_{S}\left(\mu_{R}^{2}\right)}{2 \pi}\right)^{n} f_{a}\left(x_{1}, \mu_{F}^{2}\right) f_{b}\left(x_{2}, \mu_{F}^{2}\right) w_{a b}(\Phi) O(\Phi),
$$

${ }^{1}$ Special consideration must be given to the KP contribution also in another respect. The involved linear combination of quark PDFs, cf. Eq. (3.8), only involves light quarks in SHERPA, and it must be ensured, that the number of light quarks is set consistently in MCGRID. The default in both packages is 5 , i.e. all quarks except the top quark are considered light. 
This abstraction of the dependence structure is advantageous, when an a posteriori event reweighting is employed, because for each summand, only the following set of values must be communicated: $n, \mu_{R}$ and $\mu_{F}, a$ and $b, x_{1 / 2}$ and $w_{a b}$, and $O(\Phi)$, and in addition possibly the scale logarithm coefficients $c_{R}^{\prime(0 / 1)}, \bar{c}_{F, a / b}^{(i)}$. The reweighting implementation itself only needs to evaluate Eqs. (4.2) to (4.4) for each of these value sets.

Usually one wants to know the distribution of $O$ and not its average. The weight that falls in a single observable bin $k$ of $O$ with lower and upper boundaries $O_{b}^{\min }, O_{b}^{\max }$ is given as

$$
w_{k}^{(\mathrm{N}) \mathrm{LO}}=\sum_{e} \sum_{\text {terms in } e}\left(\frac{\alpha_{S}\left(\mu_{R}^{2}\right)}{2 \pi}\right)^{n} f_{a}\left(x_{1}, \mu_{F}^{2}\right) f_{b}\left(x_{2}, \mu_{F}^{2}\right) w_{k, a b},
$$

where the binned weight $w_{k, a b}$ is defined with the help of step functions $\Theta$ as

$$
w_{k, a b}(\Phi)=w_{a b} \Theta\left(O_{k}^{\max }-O(\Phi)\right) \Theta\left(O(\Phi)-O_{k}^{\min }\right)
$$

A sum over events can still be too costly for certain applications, where speed is critical due to the amount of variations necessary, as e.g. for PDF fits. Interpolation grids overcome this by casting the sum over events to a sum over (usually much less) grid points. This is achieved by discretising the $x$ and $\mu$ values by interpolating the incoming weights $w_{a b}$ of each of the above summands to $\left(x_{1}, x_{2}, \mu\right)$ points on a fixed 3-dimensional grid. First we rewrite $\alpha_{S}$ and the pair of PDF values with the help of an interpolation kernel $\mathcal{I}$ :

$$
\alpha_{S}\left(\mu_{R}^{2}\right) f_{a}\left(x_{1}, \mu_{F}^{2}\right) f_{b}\left(x_{2}, \mu_{F}^{2}\right)=\sum_{\alpha \beta}^{N_{x}} \sum_{\tau}^{N_{\mu}} \alpha_{S}\left(\mu_{R}^{2}\left(\mu_{\tau}\right)\right) f_{a}\left(x_{\alpha}, \mu_{\tau}\right) f_{b}\left(x_{\beta}, \mu_{\tau}\right) \mathcal{I}_{\alpha \beta, \tau}\left(x_{1}, x_{2}, \mu_{\tau}\right)
$$

with the number of points $N_{x}$ and $N_{\mu}$ in the $x_{a / b}$ and the $\mu$ dimensions of the grid, respectively. In order not to introduce another grid dimension, we assumed that $\mu_{R}$ can be written as a function of $\mu_{F}$. For a detailed account on the interpolation approaches, see the APPLGRID and FASTNLO publications [51-53]. However, in Fig. 4.1, we give a visualisation of the polynomial interpolation as it is done in APPLGRID. There, the interpolation kernels $\mathcal{I}$ are polynomials of the interpolation order $n$. The interpolated function value at $x$ is given as the weighted sum over the actual function values at $n+1$ neighbouring interpolation nodes. The weights are given by the value of the interpolation kernel at $x$. In the figure, we exemplify this with the neighbouring interpolation nodes and the associated kernels used for the shaded region between two adjacent nodes, for an interpolation order of $n=3$. It can be seen, that the largest weight is assigned to the function values at the adjacent nodes, as expected.

Inserting the interpolation of QCD parameters, Eq. (4.7), allows us to rewrite Eq. (4.5) as

$$
w_{k}^{(\mathrm{N}) \mathrm{LO}}=\sum_{n} \sum_{a b} \sum_{\alpha \beta}^{N_{x}} \sum_{\tau}^{N_{\mu}}\left(\frac{\alpha_{S}\left(\mu_{R}^{2}\left(\mu_{\tau}\right)\right)}{2 \pi}\right)^{n} f_{a}\left(x_{\alpha}, \mu_{\tau}\right) f_{b}\left(x_{\beta}, \mu_{\tau}\right) W_{k, a b, n}^{\alpha \beta, \tau},
$$




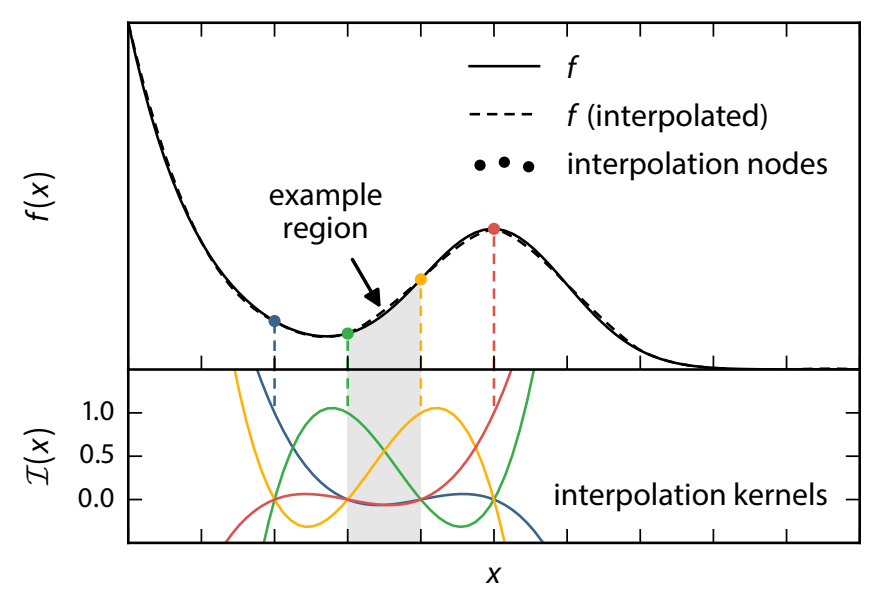

Figure 4.1.: A visualisation of the polynomial grid interpolation used in APPLgrid, for an arbitrary function $f$. Included are the interpolation nodes and kernels that are used for interpolating function values in the shaded region between two adjacent grid nodes, for an interpolation order of $n=3$.

where the sum over events has been moved into combined weights $W_{k}$ :

$$
W_{k, a b, n}^{\alpha \beta, \tau} \equiv \sum_{e} \sum_{\text {terms in } e} \delta_{a b, a b_{\mathrm{term}}} \delta_{n, n_{\mathrm{term}}} \mathcal{I}_{\alpha \beta, \tau}\left(x_{1}, x_{2}, \mu_{F}^{2}\right) w_{k, a b}
$$

The subscripts $k, a b, n$ label separate grids, and the superscripts $\alpha \beta, \tau$ specify the grid point to fill. As soon as all these grids are filled by enough events, the encoded cross section can be recombined to a new prediction for the observable via Eq. (4.8) at any later time.

\subsubsection{Scale logarithms: RGE approach vs. dedicated grids}

When we transitioned to grid weights, we did not address the issue of the scale logarithms that must be taken into account when the grids are used for scale variations.

This can either be done when the grids are filled, by using separate grids for the scale logarithm coefficients $c_{R}^{\prime(0 / 1)}$ and $\bar{c}_{F, a / b}^{(i)}$, or during the recombination by demanding that the scale derivative of the recovered cross sections results to zero to next-to-next-to-leading order in $\alpha_{S}$. Through this renormalisation group equation (RGE) approach, the missing terms can be calculated, as long as the scale variation is restricted to constant scale factors [51]. The RGE method also assumes that the interpolation grids are filled with asymptotic statistics (only then the scale derivative is expected to vanish). So, if e.g. in a closure test the grids are only filled with a small number of events, the separate grids method is expected to give a more precise closure. However, the practical reason to use separate grids is to overcome the restriction to constant scale factors. 


\subsubsection{LO \& NLO plus parton-shower calculations}

We can replace $O(\Phi)$ with $\operatorname{PS}(O, \Phi)$ in Eq. (4.1) to allow for parton-shower emissions to modify the final state. In this context, PS can denote either the ordinary shower or the

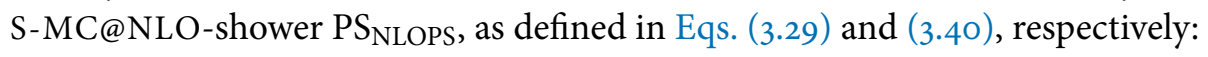

$$
\langle O\rangle^{(\mathrm{N}) \mathrm{LOPS}}=\sum_{\text {terms }} \int \mathrm{d} \Phi\left(\frac{\alpha_{S}\left(\mu_{R}^{2}\right)}{2 \pi}\right)^{n} f_{a}\left(x_{1}, \mu_{F}^{2}\right) f_{b}\left(x_{2}, \mu_{F}^{2}\right) w_{a b}(\Phi) \operatorname{PS}(O, \Phi)
$$

By doing so, we can include LOPS and NLOPS calculations in our list of calculations that can be brought into such a form, and readily fill interpolation grids. In particular, we note that the new $\mathrm{D}_{A}$ contribution in a S-MC@NLO calculation is structurally not different from the $\mathrm{D}_{S}$ one that is already found in NLO calculations. Therefore, we do not have to modify Eq. (4.10).

Although we can allow for more exclusive observables by letting the parton shower act on our final state, the interpolation grid as described above has no notion of the dependences of the shower, as has been detailed in Section 3.3, and therefore we expect that it fails to reproduce all features of a dedicated prediction. Compare for example the ME-only results with the ones where the reweighting of parton-shower emissions has been enabled for the on-the-fly reweighting validations in Fig. 3.8. We will come back to this in Section 4.6.

\subsubsection{Optimised subprocess bases}

The number of grids can be reduced and the recombination time decreased by making use of initial-state flavour symmetries. Consider e.g. Drell-Yan, where all quark-antiquark pairs share the same matrix element. The only difference is the PDF prefactor. We can therefore rewrite Eq. (4.8) as

$$
w_{k}^{(\mathrm{N}) \mathrm{LO}}=\sum_{n} \sum_{l} \sum_{\alpha \beta}^{N_{x}} \sum_{\tau}^{N_{\mu}}\left(\frac{\alpha_{S}\left(\mu_{R}^{2}\left(\mu_{\tau}\right)\right)}{2 \pi}\right)^{n} F_{l}\left(x_{\alpha}, x_{\beta}, \mu_{\tau}\right) W_{k, l, n}^{\alpha \beta, \tau} .
$$

Here, $l$ labels the lists of the flavour-symmetric subprocesses. Hence, the linear combination of PDFs associated with $l$ is defined by

$$
F_{l}\left(x_{\alpha}, x_{\beta}, \mu_{\tau}\right) \equiv \sum_{a b} C_{a b}^{l} f_{a}\left(x_{\alpha}, \mu_{\tau}\right) f_{b}\left(x_{\beta}, \mu_{\tau}\right),
$$

with $C_{a b}^{l}=1$ if $a b$ is in $l$ and 0 otherwise. For each pair $a b$, at most one $C_{a b}^{l}$ can be one by construction. Depending on the order, some pairs $a b$ might not contribute at all, then $C_{a b}^{l}=0$ for all $l$. By combining weights of subprocesses that are generated separately in the Monte-Carlo integration, the resulting grid will not be statistically equivalent to the event generation run with which it was created. This is discussed in detail in [54]. 


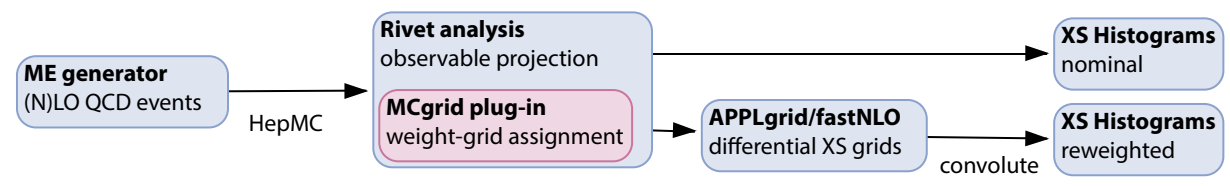

Figure 4.2.: A flow chart of an automated fastNLO/APPLgrid interpolation grid production via the MCgrid plug-in. The interpolation grids are filled alongside nominal histograms by calling MCgrid code from a Rivet analysis. After the event generation, the created grids can be convoluted to obtain reweighted predictions for these histograms.

\subsection{Automation for Catani-Seymour subtracted NLO calculations via MCgrid}

Until recently, interfaces to APPLGRID and FASTNLO were only available to specialised fixed-order matrix-element generators, as e.g. NLOJET++ [166] and MCFM [167]. Because interpolation grids are observable-specific, analysis code had to be added on a case-by-case basis to these interfaces. This has changed with the release of two new interfaces to more general Monte-Carlo event generators, AMCFAST [57] and MCGRID [54-56]. The former interfaces the fixed-order matrix-element generator MADGRAPH5_AMC@NLO [163] to APPLGRID, and employs MADANALYSIS5 [164] to provide the observable projection. It supports LO and NLO calculations.

The latter, MCGRID, uses HePMC [105] event records as an input, which are projected onto observables via the Rivet [106] analysis framework, see Fig. 4.2. In fact, MCGRID is implemented as a plug-in to RIVET, and therefore profits from its extensive library of analysis codes. On the output side, both APPLGRIDS and FASTNLO grids can be filled via MCGRID. HePMC is a widely used event output format. Being interfaced to a mere data storage format, MCGRID is agnostic with respect to the actual generator that filled the event records. However, it expects NLO events to be calculated using the CS subtraction scheme, and that the respective weight information is present in the weight container of the HEPMC event record. In addition to LO and Catani-Seymour subtracted NLO calculations, also the $\mathcal{O}\left(\alpha_{S}\right)$-expansion of NLO calculations matched to a parton shower via the S-MC@NLO [87] formalism is supported. Thus, even when the parton shower is added to NLO calculations, the filled interpolation grids are still accurate to NLO.

The automation of NLO calculations in MADGRAPH5_AMC@NLO and SHERPA, along with the existing analysis code, allows for a much more automated interpolation grid production. One additional aspect here is the subprocess basis adopted when filling the grid. Whereas codes such as NLOJET++ and MCFM uses process-specifically optimised bases, MADGRAPH5_AMC@NLO and SHERPA output fully exclusive events, i.e. with all partonic 
subchannels separate from another. This would lead to bigger memory usage of the produced grids, and also to a bigger CPU footprint, when one fills a grid and, more importantly for practical purposes, when one convolutes a grid. However, internally both generators use process-optimised subprocess bases, and both AMCFAST and MCGRID provide automated means to read out these bases and make use of them for the grid creation.

As a part of this thesis, MCGRID had been extended to support FASTNLO and the S-MC@NLO $\mathcal{O}\left(\alpha_{S}\right)$ expansion. In addition, support has been added for the dedicated scale logarithm grids in APPLGRID, adding another fill mode besides the RGE approach. We will therefore mainly validate these new features in the following section.

\subsection{Closure tests for fixed-order calculations}

To establish our set-up, we first present an auto-closure test for the tit-production process with a semileptonic final state at the LHC with a centre-of-mass energy of $7 \mathrm{TeV}$, where it is mostly sensitive to the gluon content of the incoming protons.

SHERPA NLO events are generated, with the one-loop results provided by OPENLoops. The parton shower and non-perturbative effects are disabled. The $\alpha_{S}$ and PDF values are provided by the CT1o PDF set. The scales $\mu_{F}$ and $\mu_{R}$ are set to the squared average of the transverse masses of the top quarks.

The top quarks have their mass set to $172.5 \mathrm{GeV}$, and they are produced on their mass shell and then decayed further with a zero-width approximation. First the $t$ quark decays to $\mathrm{a} \mathrm{W}^{+}$and a b/s/d quark, and the $\overline{\mathrm{t}}$ quark decays accordingly to a $\mathrm{W}^{-}$and a $\overline{\mathrm{b}} / \overline{\mathrm{s}} / \overline{\mathrm{d}}$ quark. The $\mathrm{W}^{+}$is then restricted to decay leptonically, either to a $\mathrm{e}^{+} v_{\mathrm{e}^{-}}$or a $\mu^{+} v_{\mu}$ pair. The $\mathrm{W}^{-}$, on the other hand, is set to decay hadronically. As the top quark is too heavy to be produced by an on-shell decay of the $\mathrm{W}$, this leaves the sc̄ and the dū channels. Again, the decay widths are set to zero. As we assign explicitly, which of the W-bosons decays hadronically, and which one leptonically, we multiply the resulting event weights by two, which gives us the correct result as long as the observables considered are symmetric under charge-conjugation.

The analysis reconstructs jets from the Monte-Carlo data with the anti- $k_{T}$ algorithm [107] with a jet radius parameter of $R=0.4$. At least three are required, one of which is required to be seeded by a $b$ quark. The two leading jets minimum transverse momenta cuts are $50 \mathrm{GeV}$ and $35 \mathrm{GeV}$. The MCGRID plug-in is used to fill an interpolation grid for the transverse momentum of the first jet (ordered in $p_{T}$ ). The jets consist of the real emission parton of the NLO calculation and/or the partons from the hard decays.

In Fig. 4.3, the auto-closure result for this observable is presented. The convolution from the grid reproduces the nominal result within $\mathcal{O}\left(10^{-4}\right)$ accuracy, which is of the order of typical APPLGRID interpolation accuracies [51].

To validate our method to fill the $\mathcal{O}\left(\alpha_{S}\right)$ expansion of S-MC@NLO calculations, we generate two Drell-Yan samples for $\mathrm{p}^{+} \mathrm{p}^{-}$collisions at a centre-of-mass energy of $1.96 \mathrm{TeV}$, one at NLO and one at NLOPS. To select for the $\mathrm{Z}$ resonance, an invariant mass of $66 \mathrm{GeV}<$ $m_{\ell \ell}<116 \mathrm{GeV}$ is required. The scales are fixed to the mass of the Z-boson, such that the 


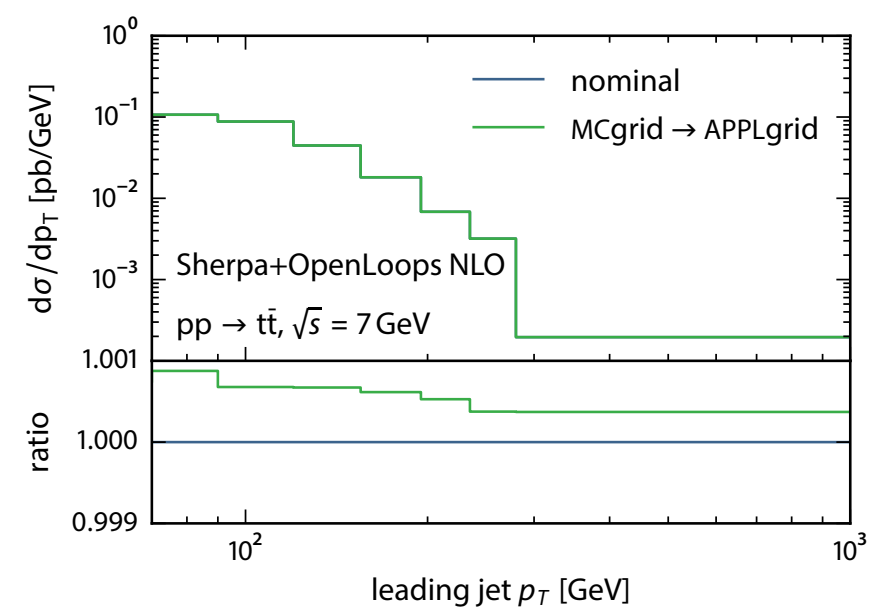

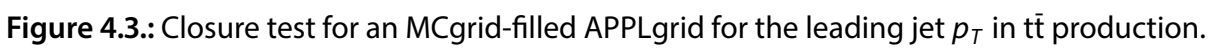

generated grid only interpolates in $x_{1}$ and $x_{2}$. To ensure statistical equivalence, all partonshower emissions in the NLOPS run are disabled. Hence, we are able to validate that the $\left(D_{A}-D_{S}\right)$-terms exclusive to the S-MC@NLO event generation are reproduced within interpolation accuracy.

We compare the deviations of the re-convolutions of both calculations with respect to their respective nominal result in Fig. 4.4. We observe, that both deviations are of a comparable size. In fact, they are much smaller than for the tit-production auto-closure test in Fig. 4.3, because the additional scale interpolation is absent in our Drell-Yan grid.

Comparing the NLO and the NLOPS event generation, we find that the latter does not fill the phase-space for $0<p_{T}^{2} \lesssim m_{Z}^{2}=\mu_{Q}^{2}$, due to the missing parton-shower emissions in this region, that would otherwise move events with $p_{T}^{2}=0$ into this region.

\subsection{Comparison of scale-logarithm methods}

We now validate our implementation within MCGRID for filling separate APPLGRIDs for the scale logarithm coefficients, as discussed in Section 4.1.2. To this end, we again generate a Drell-Yan sample with SHERPA at NLOPS for $\mathrm{p}^{+} \mathrm{p}^{-}$collisions at a centre-of-mass energy of $1.96 \mathrm{TeV}$ with $66 \mathrm{GeV}<m_{\ell \ell}<116 \mathrm{GeV}$ and the CT1o PDF set. The scales are now set dynamically to $\mu_{R}=\mu_{F}=H_{T}^{\prime}$, as defined in Eq. (3.9).

Two grids are produced for the rapidity $y$ of the $\mathrm{Z}$ boson, one including separate scale logarithm grids, and one that is using the RGE method. We then compare the reweighting for scaling $\mu_{R}$ and $\mu_{F}$ to $2 \mu_{R}$ and $2 \mu_{F}$ with a dedicated sample. We also include the result from the on-the-fly reweighting implemented in SHERPA, as discussed in Chapter 3. For all samples, parton-shower emissions are disabled to eliminate statistical fluctuations as far as possible, such that all deviations should be due to the numerical and interpolation 


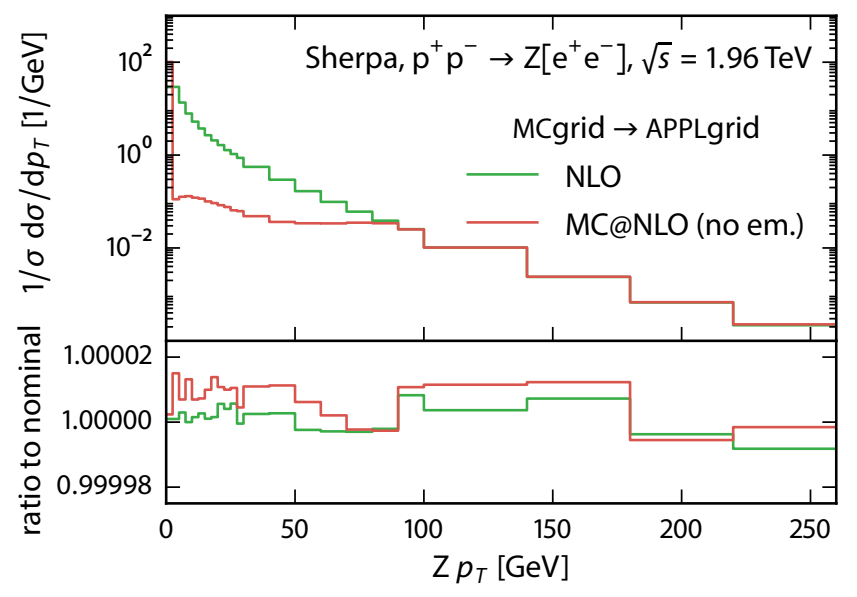

Figure 4.4.: Auto-closure tests for an MCgrid-filled APPLgrid for $p_{T}^{Z}$ in off-shell Z-boson production, with a comparison of NLO and NLOPS event generations. To enforce equivalent statistics, parton-shower emissions are disabled in the MC@NLO run. In the lower panel, the ratios to the nominal runs are shown.

accuracies. The only exception would be the RGE method, which is not expected to give a statistically equivalent result.

In fact, in Fig. 4.5 we observe that the deviations for the RGE method are about a factor of ten higher than with the separate grid method. The internal reweighting as discussed in Chapter 3 is also plotted for comparison. However, on the depicted scale, no deviations from a dedicated run is visible in this case.

\subsection{Comparison between MCgrid-filled APPLgrids and fastNLO grids}

Another MCGRID-addition developed during this thesis is the support for FASTNLO grids. In this section, we validate this new feature using auto-closure tests comparing MCGRIDfilled APPLGRIDS and FASTNLO grids.

We consider two observables for this, the hardest jet $p_{T}$ in dijet production for pp collisions and the $\mathrm{Z}$ rapidity in $\mathrm{Z}$ production in $\mathrm{p}^{+} \mathrm{p}^{-}$collisions, at a centre-of-mass energy of $7 \mathrm{TeV}$ and $1.96 \mathrm{TeV}$, respectively. For the dijets, the anti- $k_{T}$ algorithm has been used with a jet radius parameter of $R=0.5$.

We will see that APPLGRID performs slightly better in both cases. However, the comparability is not fully given, because although we ensure that the same number of grid points and the same statistics is used, the interpolation kernels and the $x / \mu$ parametrisation are different. From a phenomenology viewpoint the deviations are tiny in both cases, being much smaller than other theoretical uncertainties.

For both observables, we use SHERPA at NLO, and generate both grids in one run. The 


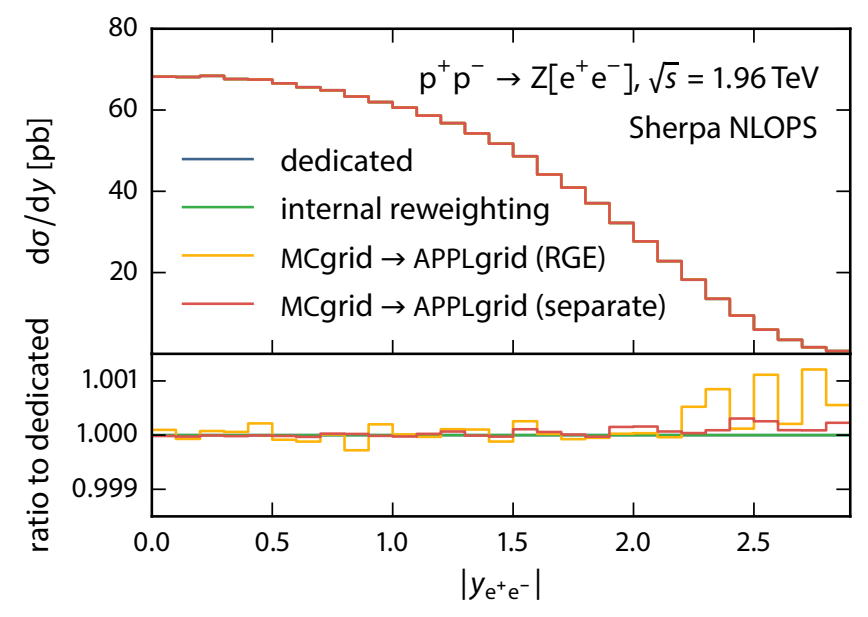

Figure 4.5.: Cross-closure tests for the Z-boson rapidity in off-shell Z production, with a nominal scale choice $\mu_{R}=\mu_{F}=H_{T}^{\prime}$, which is reweighted up to $2 H_{T}^{\prime}$. Results from MCgrid-filled APPLgrids using the separate grid method and the RGE method are compared with each other, and to a result from the internal reweighting of Sherpa.

$\alpha_{S}$ and PDF values are given by the CT1o PDF set. For the dijet production, the one-loop matrix element is provided by ВцаскНАт. In Fig. 4.6, the auto-closure tests for the two mentioned observables are presented. For both we find good agreement, the deviations in the jet $p_{T}$ case goes up to $2 \cdot 10^{-3}$ and $3 \cdot 10^{-3}$ for APPLGRID and FASTNLO, respectively. This deviation is largest for small jet $p_{T}$, where $x$ is also smallest on average. This suggests that the parametrisation of the $x$ values with respect to the internal grid space is more sparse for small $x$, such that the interpolation accuracy is worse. The other observables/processes considered so far involved the production of massive particles, such that they are less vulnerable to such a "shortcoming" in the grid architecture. However, as long as the reproduction accuracy is still considerably smaller than $1 \%$, there is no problem when it comes to physics applications. For the $\mathrm{Z}$ rapidity, we present two auto-closure tests. For the one labelled "subprocess ID enabled", the subprocess basis optimisation as described in Section 4.1.4 is used. For Z production, only 12 channels are needed then. For the "subprocess ID disabled" one, the full subprocess basis is used, i.e. $11 \times 11=121$ channels $(5$ active quark flavours and their anti-particles, plus the gluon).

We find good agreement in both cases. However, the deviations are larger when the optimisation is enabled (up to $\sim 1 \%$ for large $|y|$ ), as the combination of weights for use with a reduced PDF basis means that the interpolated result is statistically not equivalent to the nominal result it is compared to [54]. However, this only reflects the limited statistical accuracy of the grid filling run in that region, and is therefore only a problem for an auto-closure validation test, not for actual applications. The advantage of the optimised subprocess basis are the reduced time needed for the grid filling run (about $t_{\text {opt. }}=3.5$ hours instead of 

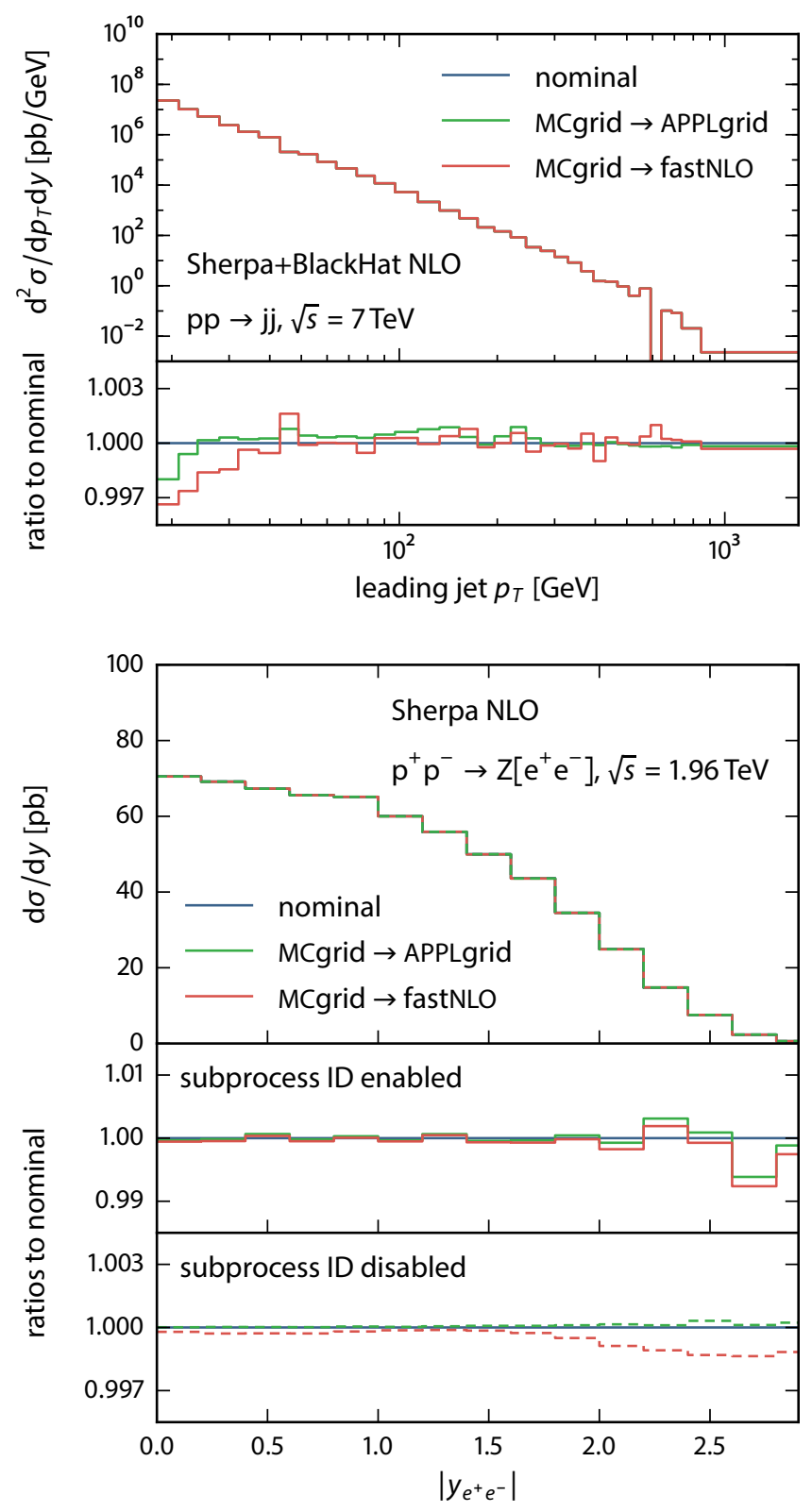

Figure 4.6.: Auto-closure tests for fastNLO grid production vs. APPLgrid production with $M C$ grid. The leading jet $p_{T}$ distribution is generated for dijet production and the rapidity $y$ of the Z-boson for off-shell Z production. For the latter, we also include results for a grid production with an optimised subprocess basis. 
$t_{\text {full }}=21$ hours in our example). When we subtract the time needed for a non-filling event generation run (about $t_{\text {baseline }}=1.5$ hours), we find a ratio

$$
\frac{t_{\text {opt. }}-t_{\text {baseline }}}{t_{\text {full }}-t_{\text {baseline }}} \approx 0.1 \text {, }
$$

which equals the ratio of channels, $12 / 121 \approx 0.1$. This suggests that the time needed for grid production scales linearly with the number of channels used.

We observe a deviation of about $0.2 \%$ between the FAsTNLO result and the nominal result for $|y|>2.0$ of, with APPLGRID performing significantly better: its deviation is below $0.1 \%$ in all bins. Again, this is only indicative of the APPLGRID defaults being better suited for the process/observable at hand.

\subsection{Comparison to the internal reweighting including the parton shower}

Finally, we come back to our claim that uncertainty bands generated from a grid should be equal to those generated by the direct reweighting approach presented in Chapter 3, provided that it is restricted to the matrix-element dependences. In other words, the number of reweighted parton-shower emissions is set to zero, $n_{\mathrm{PS}}=n_{\mathrm{NLOPS}}=0$.

To confirm this last validation, we re-use the bands we generated to test the internal reweighting at NLOPS, from an event generation for $\mathrm{W}$-boson production in pp collisions at $13 \mathrm{TeV}$, see Fig. 3.8. In Fig. 4.7, we overlay the bands generated from reweightings of an APPLGRID generated by an identical set-up via MCGRID. However, as the statistics is not equal between the reweighting and the grid-filling run, we do not calculate the ratios against a shared nominal central value. Instead, we divide the variations from the reweighting through the nominal result of the reweighting run, and the variations from the grid through the nominal result of the grid-filling run. By this, we can ignore statistical fluctuations between the two samples, and still test if the uncertainty bands come out equal. In fact, we find a perfect agreement between the two reweighting methods.

This is not to say that the grid reproduces the full uncertainty, as the dependences of the parton shower are ignored in both bands. Going back once more to Fig. 3.8, where we found that this approximation fails in the low $p_{T}$ region for the internal reweighting, we can now infer that this is also true for the grid approach.

\subsection{Future steps towards beyond fixed-order interpolation grids}

The lack of multi-order or all-order support in QCD interpolation grids puts limits on what data is usable for PDF fits, because these fits rely on the speed of the grid reweighting approach, at least beyond LO. This means that data points have to be removed from the fit, when they require resummation effects to be taken into account, or if they are better de- 

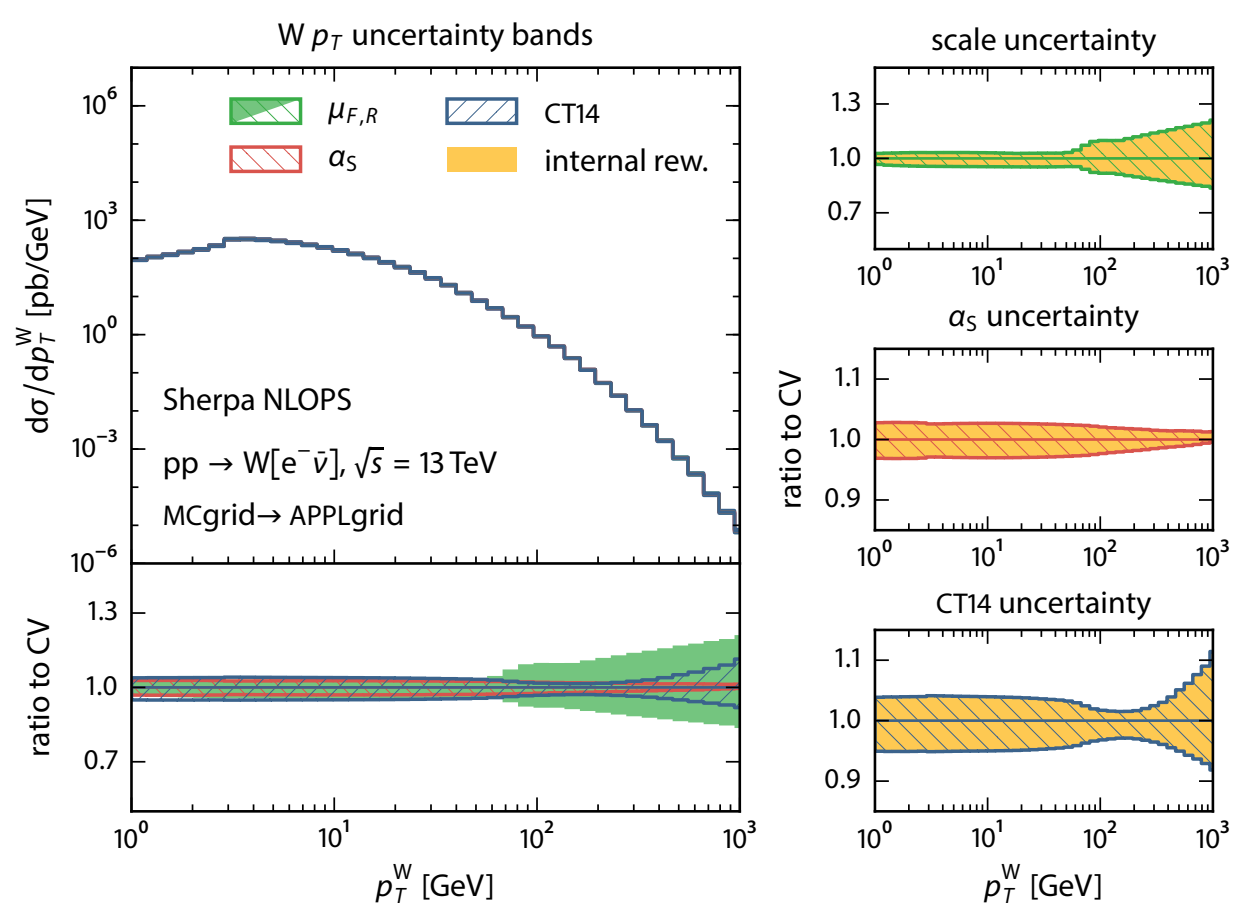

Figure 4.7.: A comparison of uncertainty bands for the $p_{T}^{\mathrm{W}}$ in a Sherpa NLOPS W production. The left-hand plot features bands that are generated by reweighting an APPLgrid filled by Sherpa via MCgrid. In the right-hand plots, these bands are compared individually to ones that are generated with the internal reweighting of Sherpa, where the reweighting of partonshower emissions is disabled.

scribed as a combined result of calculations at different jet multiplicities. All Monte-Carlo results that rely on parton showers or multi-jet merging fall into this category. Resummation effects play a role in the small- $p_{T}^{\mathrm{W} / \mathrm{Z}}$ bins, as we have seen, which is why e.g. in the NNPDF 3.o fit the corresponding data points for $p_{T}^{\mathrm{W}}$ are not used [99]. Another example is the small-angle region of the dijet azimuthal decorrelation observable, which is dominated by soft gluon emissions $[168,169]$. Both observables, $p_{T}^{\mathrm{W} / \mathrm{Z}}$ and the azimuthal decorrelation, are also examples for observables that profit from a multi-jet merged calculation. They feature overlapping regions (at large $p_{T}$ and large angles, respectively), where each one is best described by a certain number of hard jets that provide the recoil.

\subsubsection{Multi-jet merging via stacked interpolation grids}

How easy is it to modify interpolation grids to support multi-jet merged calculations? We have seen in Section 3.7 that multi-jet merged calculations feature several aspects that require a reweighting. There are the different matrix elements that can be handled individually 
as fixed-order calculations, safe for the simple PDF factors, which are replaced with several ratios of PDFs determined by the clustering, cf. Eq. (3.43). These ratios are accompanied for NLO matrix elements with appropriate counter-terms, given in Eq. (3.50), which subtract their $\alpha_{S}$ expansion to retain the NLO accuracy. The clustering also determines shower-like $\alpha_{S}$ scales, one per QCD cluster node, which are combined to determine an overall renormalisation scale, see Eq. (3.44). Morover, there are the Sudakov rejection weights, Eq. (3.46), for which truncated showers are sampled for emissions above the merging scale, which leads to the scale-/parameter-dependent veto. And finally, MENLOPS calculations come with $K$ factors, that reweight as compositions of matrix-elements, involving products and divisions, see Eq. (3.53).

Leaving aside the all-order parts for the moment, i.e. the parton shower and the related Sudakov veto, there are still elements in the remaining fixed-order calculations that can not be filled into existing interpolation grids. Matrix elements with varying multiplicities themselves are easily supported, either by implementing the support for additional sub-grids per multiplicity within APPLGRID/FASTNLO, or by operating with several APPLGRID/FASTNLO instances managed by MCGRID. Also the dynamic renormalisation scale determination by itself is not an issue, because the variation of the combined scale is correct to NLO accuracy. However, interpolation grids assume $\mu_{R}^{2}=\mu_{F}^{2}$, or at least $\mu_{R}^{2}=\mu_{R}^{2}\left(\mu_{F}^{2}\right)$, which is broken by the different prescription for the $\mu_{F}$ scale (which is determined by the lowest invariant mass or negative virtuality in the core process). Also the PDF ratios of the clustering can not be filled into interpolation grids, as they require PDFs to enter as simple prefactor pairs. On the other hand, the counter-terms only feature a single PDF factor, and the current grid implementations do not allow a mixture of single and paired PDF prefactors. The $K$ factor provides further PDF ratios, via its dependence on a $\bar{B} / \mathrm{B}$ term.

A perfect representation of these complications by a modified interpolation grid technology is a question of scalability. It is feasible to add a one grid per multiplicity and one per parton (e.g. to fill the single-PDF counter-terms), but the storage size and convolution times will increase by orders of magnitude when we begin to provide grids for each possible combination of PDF ratios, and the question of how to encode the individual cluster scales and longitudinal momentum fractions would remain.

However, one could study with a simple modification of the internal reweighting of SHERPA, how accurate it would be to neglect those complications, i.e. to only reweight the matrix-element contributions per multiplicity, and to restrict the PDF reweighting to the external incoming partons (i.e. before clustering), and to set $\mu_{F}^{2}=\mu_{R}^{2}$. At first, NLO matrix elements could be omitted to factor out the additional problems by the counter-terms and the $K$ factor.

\subsubsection{Approximate approaches to include parton-shower emissions}

The problem of a variable number of PDF ratios discussed in the previous section is also encountered when we attempt to reweight parton-shower emissions with interpolation 
grids, with the number of partonic combinations growing too large to get by with some variation of the usual approach.

One idea is motivated by our finding that some observables, such as the $p_{T}^{\mathrm{W}}$, are only sensitive to the dependences of the first two or three hardest emissions. However, even then the number of rejected emissions still varies and will be larger than the number of accepted ones. Also, the dependence structure is different between the two, which prevents a unified handling, effectively doubling the number of grids again. Hence, the problem is even more complex than with the cluster PDF ratios of the merging.

Therefore, a second idea is to not track the dependences of the parton shower at all, and instead have a number of grid replica, each one filled by events from a matrix-element plus parton-shower generation, where some scale or parameter used is varied. Then the respective parton-shower dependence is encoded in the difference between the predictions of those grid replica, and one could interpolate between those predictions. Unfortunately, even if this can be done for a single-valued parameter like $\alpha_{S}\left(m_{Z}^{2}\right)$, there is no universal ordering between different PDFs. The approach would only be feasible, if a PDF can be parametrised by a small number of values. If we look at the MMHT2014 parametrisation, there are about 40 independent parameters entering its fit. So if one wants three grid replicas per PDF parameter-a central one along with an up and a down variation, a total of 120 grids is needed. An increase of the number of grids by two orders of magnitude seems impractical in terms of storage and CPU time requirements.

In conclusion, a direct encoding of emission dependences is made infeasible by the large number of PDF ratio combinations and the duplication by the differing dependence of rejected trial emissions, and an indirect and approximate encoding by grid replicas is difficult due to the large number of degrees of freedom in PDF determinations.

\subsection{Discussion}

In this chapter we have discussed new developments for PQCD reweighting using interpolation grids, and validated them with closure tests.

Typical applications for interpolation grids are parameter fits that use an iterative approach, such as PDF fits. In these, the exact variations are not known beforehand, and the associated predictions must be calculable within milliseconds due to the large number of iterations needed. Currently, interpolation grids allow for variations of the PDFs, $\alpha_{S}\left(m_{\mathrm{Z}}^{2}\right)$, and both $\mu_{F}$ and $\mu_{R}$, for fixed-order LO, NLO and even NNLO calculations.

We have presented and validated new features in the MCGRID interface, which led to its 2.o release. First, FAsT NLO interpolation grids are now supported, such that the two main interpolation grid implementations can be used. Through this common interface, they can be compared in a reliable manner. Also, their feature sets are not equal, such that it can be advantageous to use one or another. For example, FAST NLO in principle supports NNLO cross sections, and allows for two additional sub-grids for encoding kinematic variables for a more flexible scale variation based on an arbitrary function of these two variables [53]. 
MCGRID does not yet support these two features, but especially the addition of NNLO calculations will certainly be relevant for future applications, and would be a natural next step of the development. A second addition to MCGRID we presented is the support for dedicated APPLGRIDs for the scale logarithm coefficients, which allow for scale variations that go beyond simple scale factors. Thirdly, we discussed the added support for the $\mathcal{O}\left(\alpha_{S}\right)$ expansion of S-MC@NLO calculations, by filling their $\left(\mathrm{D}_{A}-\mathrm{D}_{S}\right)$ contribution.

This last feature is a first step beyond encoding fixed-order calculations in interpolation grids. However, proper resummation effects by the inclusion of parton-shower emissions are still absent. A precondition for adding these is a precise understanding of their dependences, which we achieved in the previous chapter with our detailed account on this subject in the context of the internal reweighting. However, there are still major difficulties to overcome, such as variable numbers of PDF ratios. These need to be solved, possibly by an approximate account, before a reweighting of parton-shower emissions in interpolation grids is in reach. For now we suggest to use the on-the-fly reweighting as a fast exploratory method before the actual grid production, to assess the validity of doing fixed-order variations only. Similarly, one could assess with a modification of the on-the-fly reweighting the viability of a future implementation for an approximate handling of multi-jet merged calculations within interpolation grids. Hence, internal-reweighting methods can play a guiding role in future interpolation-grid developments. 


\section{QCD uncertainties and jet rate extrapolations at a future $100 \mathrm{TeV}$ proton-proton collider}

After the presentation of more general methods to get many predictions out of one calculation, we eventually discuss in this chapter a method to extrapolate predictions for multi-jet rates to higher jet multiplicities.

This is presented in the context of a more general exploration of multi-jet physics at a highmultiplicity environment, namely a $100 \mathrm{TeV}$ pp collider. Such a machine is under discussion by the high-energy physics community as an important next step after the completion of the physics programmes at the LHC and the High-Luminosity LHC $[5,6]$, with an anticipated luminosity of the order of $1-10 \mathrm{ab}^{-1}$ [6-8]. It is called the Future Circular Collider (FCC).

As for the LHC, we will need a good description of the Standard Model of particle physics (SM) at the FCC, because also beyond-SM particles eventually decay to SM particles in the final state within most models, and because beyond-SM interactions could influence the production of SM particles. This means that the SM provides both potential backgrounds and signatures for the exploration of beyond-SM phenomena. In addition, the FCC will provide sizeable rates even for rather rare processes, as e.g. rare decays of top-quarks or Higgs-bosons. This can be used to improve the precision in the determination of SM parameters, and to look for deviations from SM dynamics at distances that are even shorter than at the LHC. Besides being eventually used as precision tools during the data analysis, Monte-Carlo event generators can be used as exploratory tools in the planning phase of such a future collider, to calculate estimates for these interesting backgrounds and signals.

Here, we focus on the huge amount of multi-jet activity that is expected at the FCC. This opens up many possibilities for searches of exotic physics beyond the SM such as black holes or instantons decaying into jets. A large number or events containing systems with effective masses of 10 or even $20 \mathrm{TeV}$ would be observed which will also explore a region where no prior experience of QCD exists. In view of the enormous phase space available for producing additional jets at the FCC, new methods to estimate and probe the tails of the jet multiplicity distribution are desirable, in a region where otherwise one has to largely rely on parton-shower simulations alone.

One way to address this challenge of describing high-multiplicity jet environments within QCD is by fitting the scaling behaviour of such processes. In particular, we look at pure QCD multi-jet production and vector boson production associated with jets as typical benchmarks for this part of SM dynamics. We discuss the scaling in jet multiplicities between 14 
and $100 \mathrm{TeV}$, and the scaling behaviour in ratios of multi-jet cross sections, which differ by one in the jet multiplicity. The latter is particularly interesting, since increasing collider energies allow increasingly more hierarchical kinematics, which in turn trigger the transition of the well-known Staircase scaling —also known as Berends scaling — to a Poissonian scaling. The latter is usually associated with the onset of practically unconstrained, independent emission patterns. These scaling patterns have recently been explained using semianalytical methods $[65,170,171]$. We find that both scaling patterns are well-instantiated in our Monte-Carlo predictions, and potentially allow to extrapolate the rates for up to about 15 jets or even higher, using the first few jet bins only. Using such scaling-based predictions, background subtractions for New Physics signatures resulting from decays of new heavy coloured particles yielding a distinct imprint on the multiplicity distribution might become feasible $[62,172]$.

We discuss the jet activity to be expected at the FCC in Section 5.1, and explore jet scaling patterns and their extrapolations in Section 5.2. Parts of this chapter have been published within a more extensive study carried out to assess SM physics at the FCC as a step towards a Conceptual Design Report [173], and within a paper that discusses a variety of pQCD aspects in an FCC environment [174].

\subsection{QCD cross sections at high energies}

In order to gain some intuition about the kind of known physics that will be encountered at such a machine, representative cross sections for the production of relevant highmultiplicity final states are compiled in Fig. 5.1. As in all studies in this chapter, the anti- $k_{T}$ algorithm [107] is used to cluster jets with a radius parameter of $R=0.4$. The first thing to notice is the inelastic cross section at the FCC, being around $105 \mathrm{mb}$ [175], which constitutes a $45 \%$ raise compared to the LHC $(\sim 72 \mathrm{mb})$. To contrast that, we calculated LO cross sections for a multitude of processes, with cross sections ranging from a few attobarn up to hundreds of microbarn, across 15 orders of magnitude. QCD-only processes come with the largest cross sections when a jet cut of $p_{T, \text { min }}=50 \mathrm{GeV}$ is used, with dijet production at $315 \mu \mathrm{b}$. Also higher jet multiplicities have very high cross sections, and only inclusive 7-jet production is less probable than any other hard process: The inclusive single vector boson production cross sections come with $350 \mathrm{nb}-600 \mathrm{nb}$ and are thus slightly enhanced compared to 7-jet production. The least probable cross sections included in Fig. 5.1 are those of triple Higgs production in association with a vector boson or from vector-boson fusion with at least two jets. These cross sections are between 3 and $20 \mathrm{ab}$. Thus at least the $H^{3} j^{2}$ cross section would still correspond to several events at a luminosity of $1-10 \mathrm{ab}^{-1}$.

The total inclusive cross sections are compared to those at $14 \mathrm{TeV}$ in Fig. 5.2a where one clearly sees the increasing multiplicity of events at the higher centre-of-mass energy. Turning our attention to the $p_{T}$ spectra, in Fig. 5.2b we show cumulative distributions for a democratic cut of $1 \mathrm{TeV}$ on all jets for the first 6 jets ordered in $p_{T}$. This sample now has been generated using SHERPA with a MEPS@LO set-up with matrix elements for up to two 


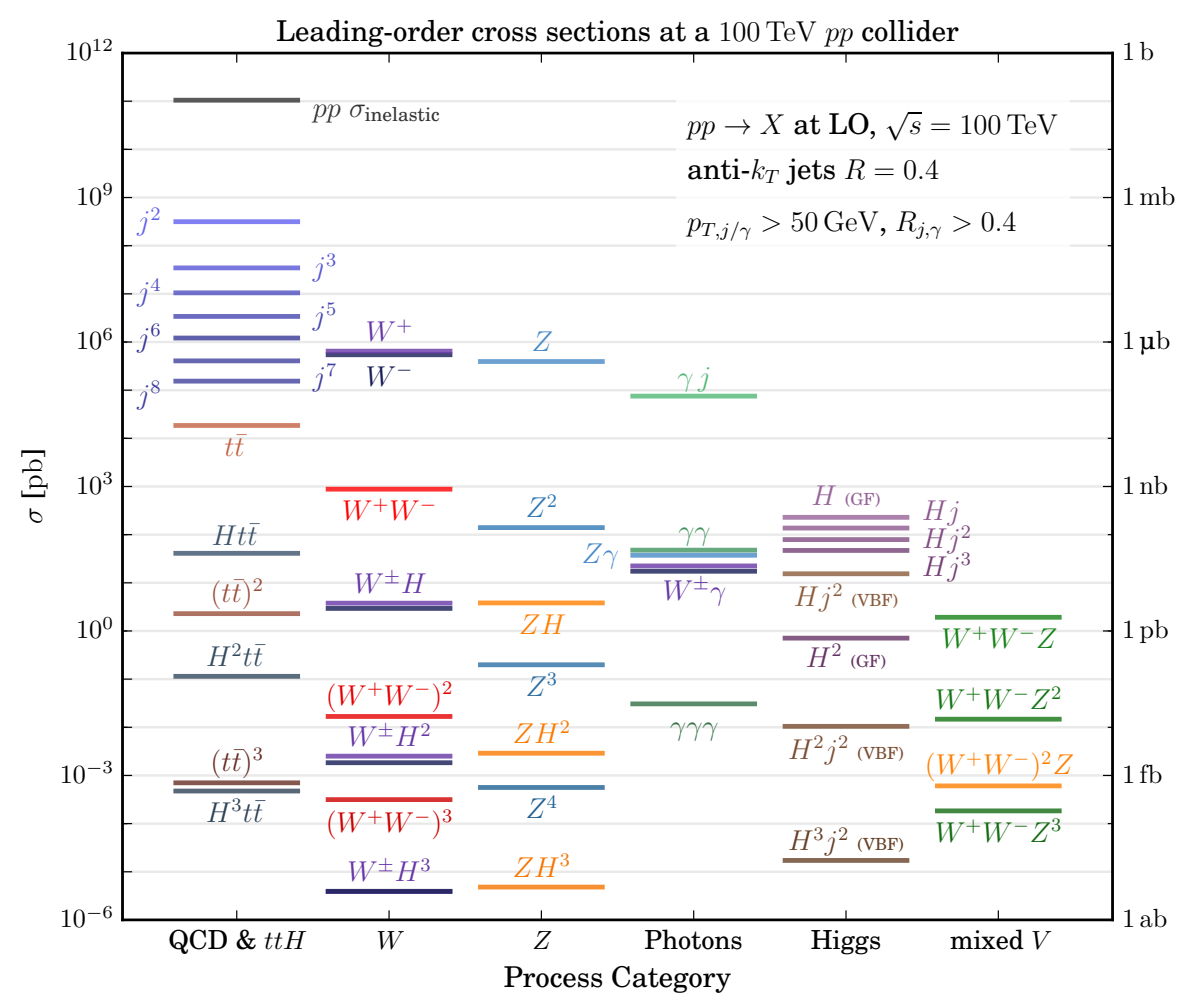

Figure 5.1.: A compilation of LO cross sections at the $100 \mathrm{TeV}$ future proton-proton collider. The Higgs cross sections labelled with "GF" refer to Higgs production via gluon fusion, whereas "VBF" stands for vector-boson fusion production. For gluon-fusion Higgs production the top-mass effects have been included.

additional jets on top of the dijet core process merged together and dressed with parton showers. Though the energy distribution for the highest multiplicity jets fall quickly, many events will be observed where the 6 th jet has still more than $400-500 \mathrm{GeV}$. The leading jets are accessible at energies much greater than $3 \mathrm{TeV}$.

In Fig. 5.3 jet rates at NLO QCD differential in jet transverse momentum and additionally binned in jet rapidity $y$ are presented. These results have been obtained with BLACKHAT+SHERPA [77]. The renormalisation and factorisation scale have been set to $\mu_{R}=\mu_{F}=$ $H_{T} / 2$. For the definition of $H_{T}$, cf. Eq. (3.54). Comparing rates for 14 and $100 \mathrm{TeV}$ centreof-mass energy an increase of about one order of magnitude for central jets with low and moderate $p_{T}$ is observed. Considering larger $p_{T}$ values the differences get more extreme, at $p_{T}=3.5 \mathrm{TeV}$ the FCC rates are more than three orders of magnitude larger than at the LHC. In fact, the FCC provides substantial jet rates even for very large rapidities: $200 \mathrm{GeV}$ jets with $5<|y|<6$ come with rates about two orders of magnitude larger than those for $200 \mathrm{GeV}$ jets in the $4<|y|<5$ bin at the LHC. From these rate estimates it can be concluded 


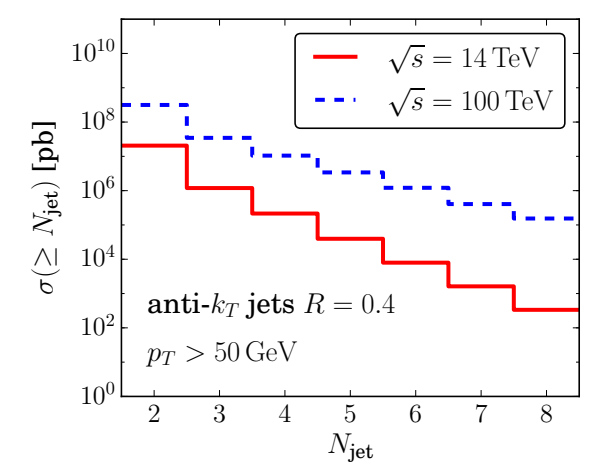

(a) A comparison for inclusive jet multiplicities $N_{\text {jet }}$ between collisions at $\sqrt{s}=14 \mathrm{TeV}$ and $\sqrt{s}=100 \mathrm{TeV}$, calculated at LO. The scales are set to $\mu_{F, R}=\bar{H}_{T}$.

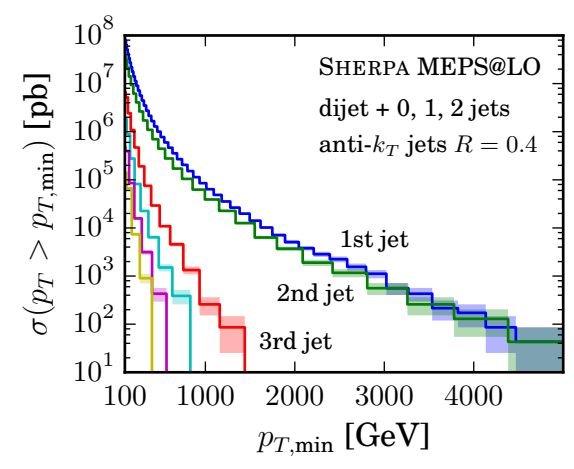

(b) CumulativeMEPS@LO $p_{T}$ distributions for the first six highest $p_{T}$ jets, ordered in $p_{T}$, at $\sqrt{s}=100 \mathrm{TeV}$. The labels for the 4th through 6 th jet are omitted.

Figure 5.2.: Jet cross sections for dijet production in proton-proton collisions.
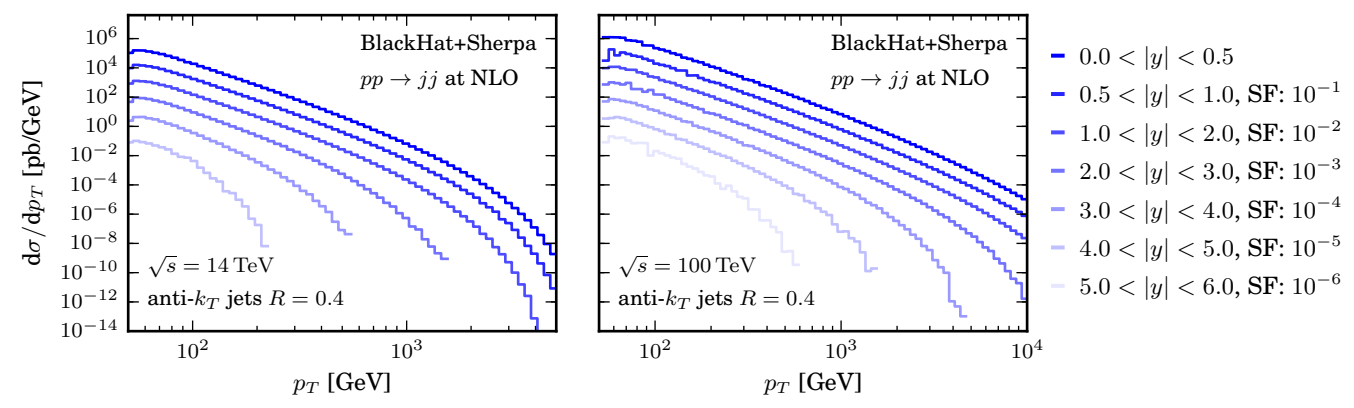

Figure 5.3.: NLO QCD inclusive jet cross sections for $\sqrt{s}=14 \mathrm{TeV}$ (left) and $\sqrt{s}=100 \mathrm{TeV}$ (right), differential in $p_{T}$ for different bins in jet rapidity $y$. Note that for illustrative purposes the results have been multiplied by variable scaling factors (SF), as indicated in the legend.

that one can expect at least ten times more jets at the FCC compared to the LHC, and this factor gets larger when looking into high $p_{T}$ and/or high $|y|$ regions or demanding large jet multiplicities. Accordingly, the rapidity coverage of general-purpose detectors at the FCC should increase with respect to ATLAS or CMS.

At NLO, we can study the reduction of the scale uncertainties in jet $p_{T}$ spectra compared to LO. In Fig. 5.4, we show NJET+SHERPA [80] predictions for the first and second leading jets ordered in $p_{T}$. Variations in the factorisation and renormalisation scale choices at NLO leads to the expected reduction in theoretical uncertainty-in this case around $10 \%$ at NLO, compared with $20 \%$ at LO.

To summarise the LO results in this section we collect a number of multi-jet QCD processes in Fig. 5.5. For four different values of the minimum $p_{T}$ we show pure jet productions with up to 8 jets and single photon with up to 7 jets. As a comparison we also show top pair 

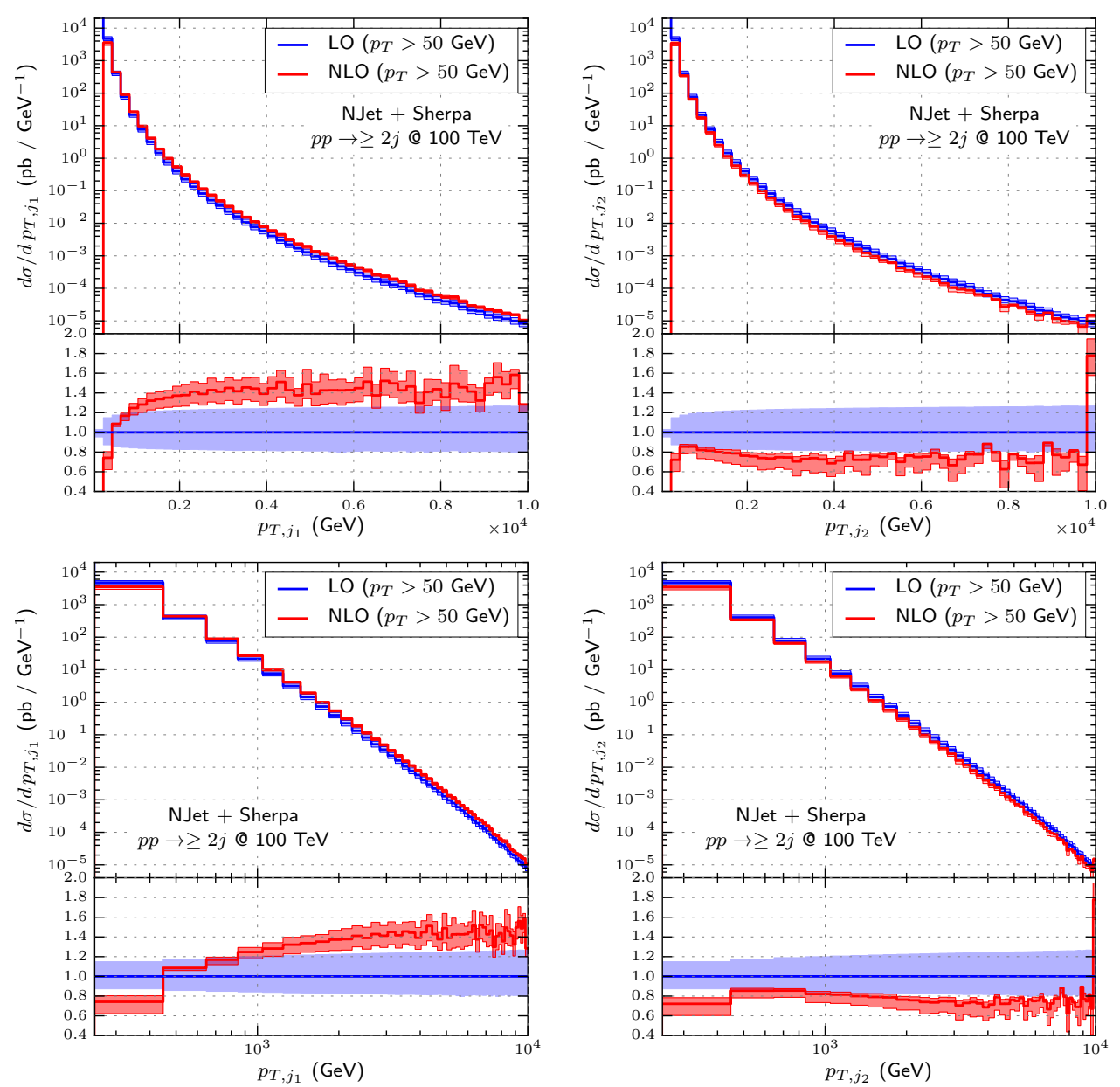

Figure 5.4.: The first and second leading jet $p_{T}$ for dijet production. LO and NLO scale variations in the range $[1 / 2,2]$ are shown around the central scale of $\mu_{R}=\mu_{F}=H_{T} / 2$. The top row shows a linear scale from $50 \mathrm{GeV}$ to $10 \mathrm{TeV}$ while the bottom row shows the same plot using a logarithmic scale over the range $250 \mathrm{GeV}$ to $10 \mathrm{TeV}$ in order to avoid the singularity which affects the first bin. 


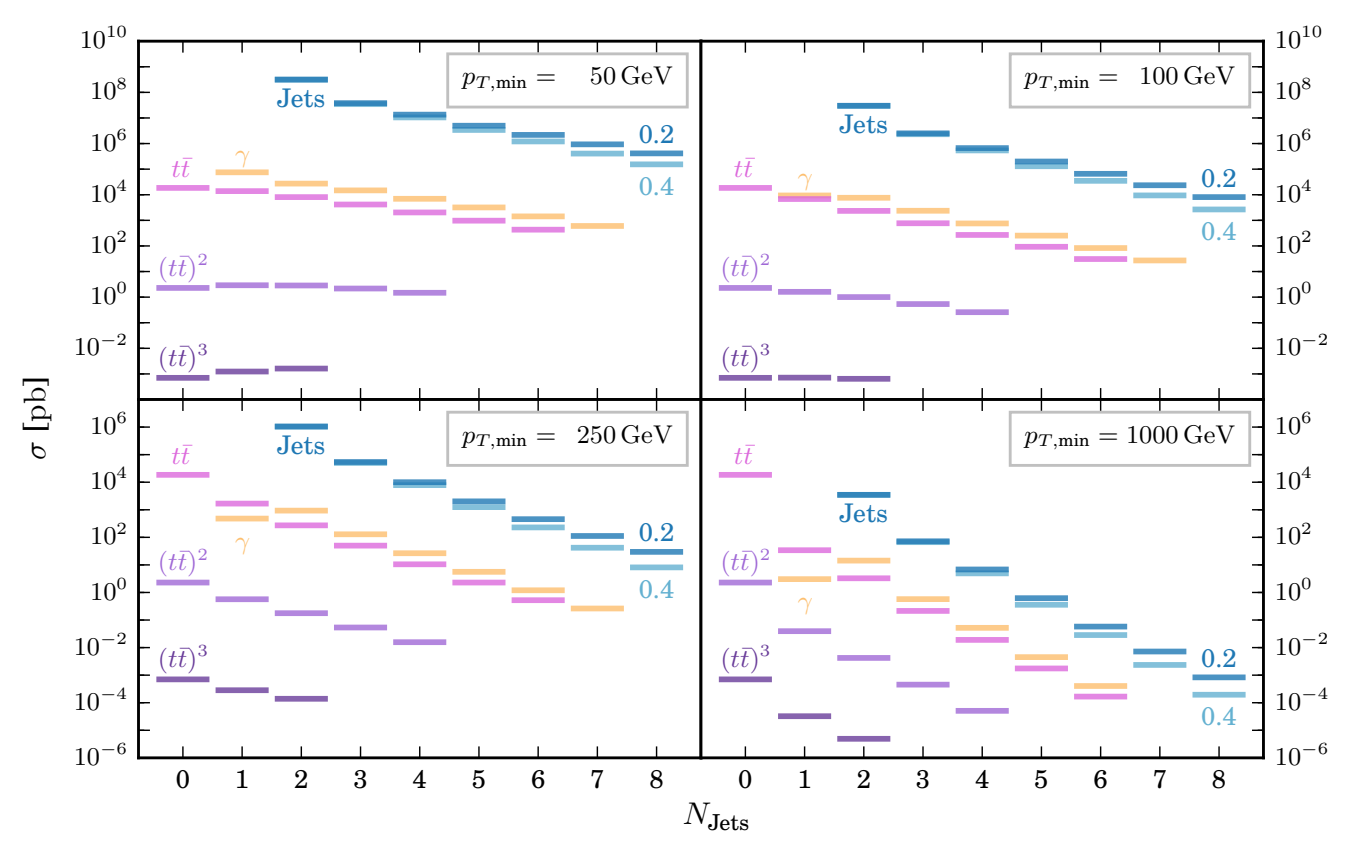

Figure 5.5.: A comparison of inclusive jet rates between various QCD processes calculated at LO for different $p_{T, \min }$. For pure jets production, an additional comparison is made between jets with radii $R=0.2$ and $R=0.4$.

production with up to 6 jets, two quark pairs with up to 4 jets and three top pairs with up to two jets. The fact that the latter processes are accessible with relatively high- $p_{T}$ jets impressively demonstrates the degree to which QCD can be studied in the $100 \mathrm{TeV}$ environment, opening up huge amounts of phase space for new physics searches.

\subsection{The scaling behaviour of multi-jet rates}

We have seen in the previous section that when considering hadron collisions at highest energies QCD jet production processes are omnipresent. Even processes with a very large multiplicity of (associated) jets exhibit sizeable rates. Accurate predictions for such final states pose a severe challenge for Monte-Carlo event generators. Fixed-order calculations are feasible only up to final-state multiplicities of 6 at NLO [127] or up to 12 at LO [70]. Beyond this point the parton shower has to be employed, which does not extend to the hardemission phase space, and therefore fails to give accurate jet-rate predictions. Therefore one might have to resort to approximate methods. This section focuses on one such approach, which is based on the scaling behaviour of QCD jet rates with respect to jet multiplicities.

The QCD jet production rates anticipated at the FCC demand suitable theoretical methods even for very large jet multiplicities. While a fixed-order prediction for a given jet process is 
suitable to describe the corresponding jet multiplicity bin, matrix-element parton-shower merging techniques provide inclusive predictions, differential in the jet multiplicity, with high jet multiplicities being modelled through the parton shower. Alternatively, there has recently been progress in making (semi-)analytical predictions for jet rates at hadron colliders that account for small jet radii and high jet counts [65, 170, 171].

With the advent of such methods, the morphology of the entirety of the jet-multiplicity distribution can be studied. Guided by phenomenological evidence, and supported by both fixed-order calculations and parton-shower simulations, certain jet-multiplicity scaling patterns can be identified [66] that find their analogue in the analytical jet-rate predictions [65, $170]$.

As already visible in Fig. 5.2a, jet rates binned in the number of jets exhibit a high degree of regularity. To study this feature one considers the ratio $R_{(n+1) / n}$ of the exclusive $(n+1)$ over the $n$-jet cross section,

$$
R_{(n+1) / n} \equiv \frac{\sigma_{n+1}^{\text {excl }}}{\sigma_{n}^{\text {excl }}} .
$$

The approximately equal step size (on a logarithmic scale) between the subsequent exclusive jet rates observed in Fig. $5.2 \mathrm{a}$ translates into a flat plateau for $R_{(n+1) / n}$, i.e. $R_{(n+1) / n} \sim$ const. This suggests a simple exponential form of the jet-rate distribution. This shape of the jet rates is called Staircase Pattern. Another regularity in jet rates found is named Poisson Pattern. This pattern is caused by jet cross sections that follow a simple Poisson statistics, resulting in $R_{(n+1) / n} \sim \bar{n} /(n+1)$, with the average number of jets given by $\bar{n}$.

Both patterns have been observed in LHC data [58-61] and in Monte-Carlo studies [6264]. They can be understood as the limiting cases for the jet-emission probability: for $\alpha_{S} / \pi \log ^{2} Q / Q_{0} \ll 1$, a Staircase Pattern is expected, while for the opposite regime, i.e. $\alpha_{S} / \pi \log ^{2} Q / Q_{0} \gg 1$, one expects a Poisson Scaling [65-67]. Here $Q$ denotes the hard process scale and $Q_{0}$ is of the order of the jet-resolution scale, i.e. $Q_{0} \sim p_{T \text {,min }}$. The derivation is based on the language of generating functionals for the jet rates. The two distinct regimes correspond to additional parton emissions being distributed either equally among all other partons or stemming predominantly from a single hard parton line. The latter follows a simple Sudakov decay-like model which results in a Poisson distribution, as is the case for photon emissions from a hard electron line [68]. The case of democratic emissions (mainly gluons from gluons) on the other hand is exclusive to field theories with a non-abelian group structure such as QCD.

In realistic measurements jet patterns will be overlaid and cut off by other effects, such as phase-space constraints. When the available energy for further jet emission is being depleted or when the jets already radiated cover a good fraction of the available solid angle [67], then higher multiplicities will quickly tend to zero. On the other hand, the first few emissions carry away sizeable parts of the total energy available, such that the increase in the partonic momentum fractions at which any participating PDFs are evaluated is comparably large. This leads to somewhat steeper decrease of jet rates for the first few emissions and is known as the PDF suppression effect [66]. 
Table 5.1.: The jet-cut scenarios considered for pure jet production at FCC energies. Furthermore, the fit hypotheses, cf. Eq. (5.2), and the corresponding parameters are listed.

\begin{tabular}{lrrlll}
\hline & \multicolumn{2}{c}{$p_{T, \text { min }}$ cuts $[\mathrm{GeV}]$} & & & \\
\cline { 2 - 5 } label & leading & other & fit function & fit region & fit parameters \\
\hline S1 (democratic) & 100 & 50 & $f_{\text {Staircase }}$ & $3 \leq n \leq 5$ & $c=0.342, m=0.006$ \\
S2 (democratic) & 200 & 100 & $f_{\text {Staircase }}$ & $3 \leq n \leq 5$ & $c=0.274, m=0.003$ \\
P1 (hierarchical) & 500 & 50 & $f_{\text {Poisson }}$ & $1 \leq n \leq 5$ & $\bar{n}=2.21, c=0.16$ \\
P2 (hierarchical) & 2000 & 50 & $f_{\text {Poisson }}$ & $1 \leq n \leq 5$ & $\bar{n}=2.64, c=0.25$ \\
\hline
\end{tabular}

To study in how far simple jet scaling patterns describe the jet multiplicity distributions at FCC energies, fits of $R_{(n+1) / n}$ in Monte-Carlo predictions are considered. For that purpose ShERPA Monte-Carlo samples for pure jet production are explored, triggering scaling patterns using either democratic or hierarchical, i.e. staggered, jet cuts. As mentioned before, democratic reflects the fact that all jet $p_{T \text {, min }}$ are of the same order, i.e. uniform, whereas hierarchical refers to the scenario where the cut on the leading jet, $p_{T, \min }^{\text {leading }}$, is significantly increased.

Both cut scenarios considered for pure jet production are listed in Table 5.1. In all cases the $2 \rightarrow 2$ core process has been considered at MC@NLO accuracy, furthermore LO matrix elements for final-state multiplicities up to six partons are included, all consistently merged with the parton shower. In Fig. 5.6, the resulting $R_{(n+1) / n}$ distributions are presented for the four considered selections. Note that the index $n$ counts the number of jets radiated off the hard two-to-two core, e.g. $n=1$ corresponds to the production of three final-state jets.

As discussed in [63], jets assigned to the core process behave differently from jets emitted thereof, which is why they have to be dismissed from pattern fits through the data. Furthermore, PDF effects leave a non-universal imprint on the first few bins. Therefore, the Staircase-pattern fits for the democratic cut scenarios are based on the values from $R_{4 / 3}$ through $R_{6 / 5}$, cf. the two upper panels of Fig. 5.6. For the hierarchical cut scenarios, the PDF suppression effect is less prominent, due to the hard cut on the leading jet that induces a much higher scale $Q$ for the core process. Accordingly, the fits for the Poisson-like patterns are based on $R_{2 / 1}$ up to $R_{6 / 5}$, cf. the two lower panels in Fig. 5.6. To quantify the quality of the fits, a term linear in $n$ for the Staircase pattern and a constant term for the Poisson pattern have been added to the ideal scaling hypotheses. Hence, the fit functions for the two scenarios are

$$
\begin{aligned}
f_{\text {Staircase }}(n) & =c+m n, \\
f_{\text {Poisson }}(n) & =\frac{\bar{n}}{n+1}+c .
\end{aligned}
$$

All resulting fit parameters are listed in Table 5.1. For all cut scenarios the fit function 


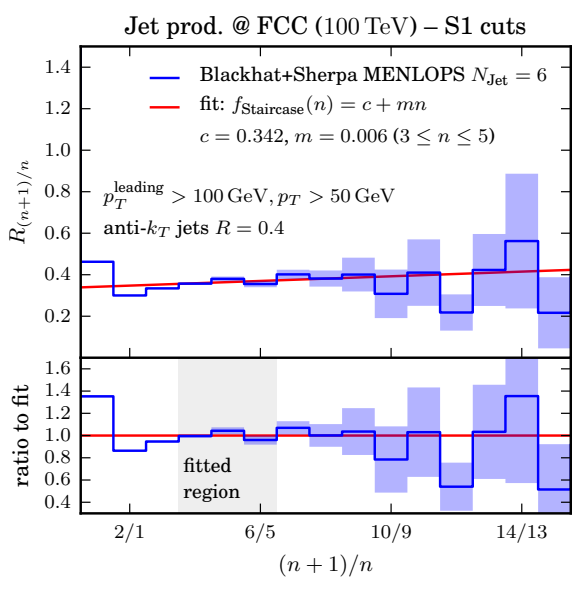

(a) S1 cuts

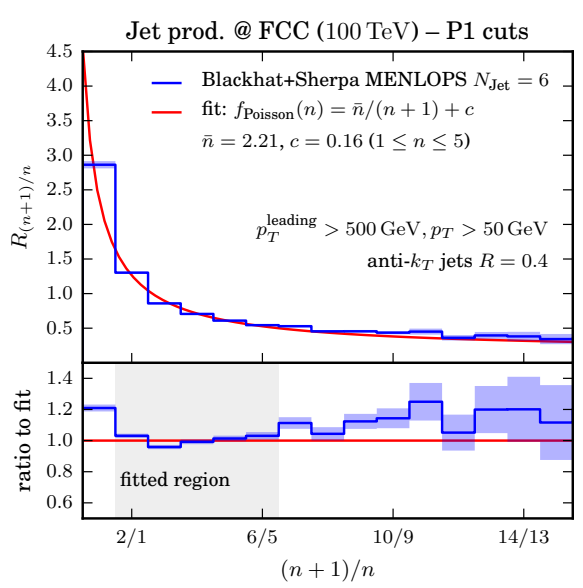

(c) P1 cuts

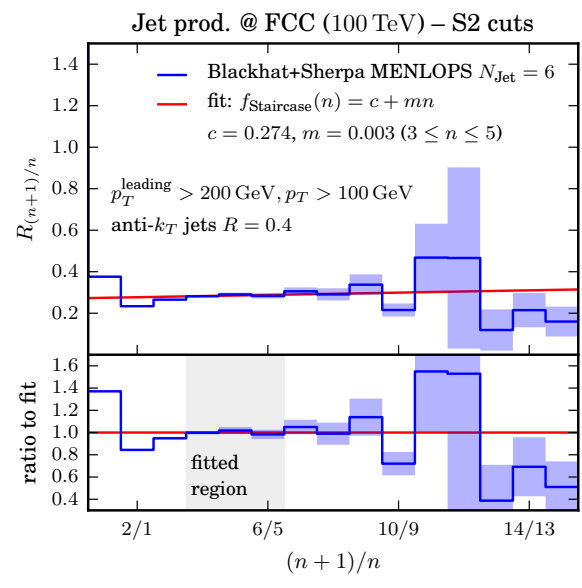

(b) S2 cuts

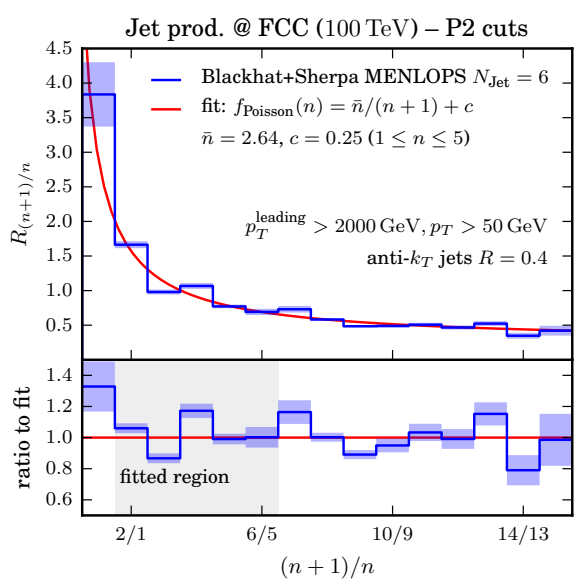

(d) P2 cuts

Figure 5.6.: The exclusive jet multiplicity ratio $R_{(n+1) / n}$ in pure jet production at the FCC. Results are presented for the four cut scenarios described in Table 5.1, with fits for the Staircase and Poisson patterns, cf. Eq. (5.2). 
and its extrapolation to higher jet bins describe the simulated data very well. For the two democratic scenarios, the constant $c$ decreases from 0.35 to 0.29 when we increase the jet cuts, reflecting the fact that the cost in terms of available energy for adding an additional jet increases.

Poisson patterns are obtained when hierarchical cuts are applied. Although the constant offset $c$ increases from 0.16 to 0.25 when enlarging the gap between the leading jet cut and the overall jet cut $p_{T, \min }$, one can see by eye that the overall fit quality is better for the larger cut gap, i.e. $2000 \mathrm{GeV}$ vs. $50 \mathrm{GeV}$. For the smaller cut gap, i.e. $500 \mathrm{GeV}$ vs. $50 \mathrm{GeV}$, the fit increasingly underestimates $R_{(n+1) / n}$ for growing $n$, which might indicate a faster transition to a Staircase-like behaviour. As expected, the average jet multiplicity $\bar{n}$ found from the fit increases with a larger leading jet cut (from 2.2 to 2.6). In particular the $S_{2}$ and $\mathrm{P}_{2}$ cut scenarios are very well modelled by the simple scaling pattern hypotheses and allow for reliable extrapolations where explicit calculations based on fixed order or even parton-shower simulations become computationally infeasible.

As explained above, jet-multiplicity scaling patterns are a generic feature of associated jet-production processes. To illustrate this, vector-boson production, and in particular $\mathrm{W}$-boson production, in association with jets will be considered in the following. Once again, samples based on an MC@NLO simulation of pp $\rightarrow$ W merged with additional LO matrix elements for up to five jets dressed with parton showers have been produced. In Fig. 5.7, the predictions for exclusive jet rates imposing a jet cut of $p_{T \text {, min }}=50 \mathrm{GeV}$ and variable cuts on the leading jet (left panel) or on the W-boson (right panel) transverse momentum are presented. The two cut schemes induce very similar shapes on the multiplicity distributions, but the overall rates are significantly smaller when demanding the W-boson to have large transverse momentum. In fact, a sizeable part of the $\mathrm{W}+$ jets cross section originates from hard jets accompanied by a vector boson with comparatively low transverse momentum [176].

For comparison, Fig. 5.8 shows the same event selections, but for a pure MC@NLO simulation of the inclusive vector-boson production process, i.e. without any additional tree-level matrix elements taken into account. Noticeably, with the lack of higher-multiplicity matrix elements the rate estimates for the high-multiplicity bins are orders of magnitude smaller than in the merged run. From similar comparisons at LHC energies, it is apparent that the predictions based on higher-multiplicity matrix elements are more reliable and describe data much better, see for instance [59-61, 177].

In Figs. 5.9 and 5.10, the exclusive jet multiplicity ratios $R_{(n+1) / n}$ for the multi-jet merged sample described above are plotted alongside with fits following the functional forms given in Eq. (5.2). In this context, the jet multiplicity, $n$, counts the number of jets in addition to the core process $\mathrm{pp} \rightarrow \ell \bar{v}_{\ell}+\mathrm{j}$, i.e. W production in association with at least one jet. In Fig. 5.9, results for the democratic selection scenario, i.e. a universal jet cut of $p_{T, \min }=50 \mathrm{GeV}$, requiring $p_{T}^{\mathrm{W}}>100 \mathrm{GeV}$, are presented. A fit of the Staircase hypothesis in the range $1 \leq n \leq 5$ results in an almost vanishing parameter $m$. This presents an ideal Staircase scaling, with a constant ratio of $c=0.4$. The extrapolation of this scaling function to higher values of $n$ is in very good agreement with the Monte-Carlo simulation results. In Fig. 5.10, 


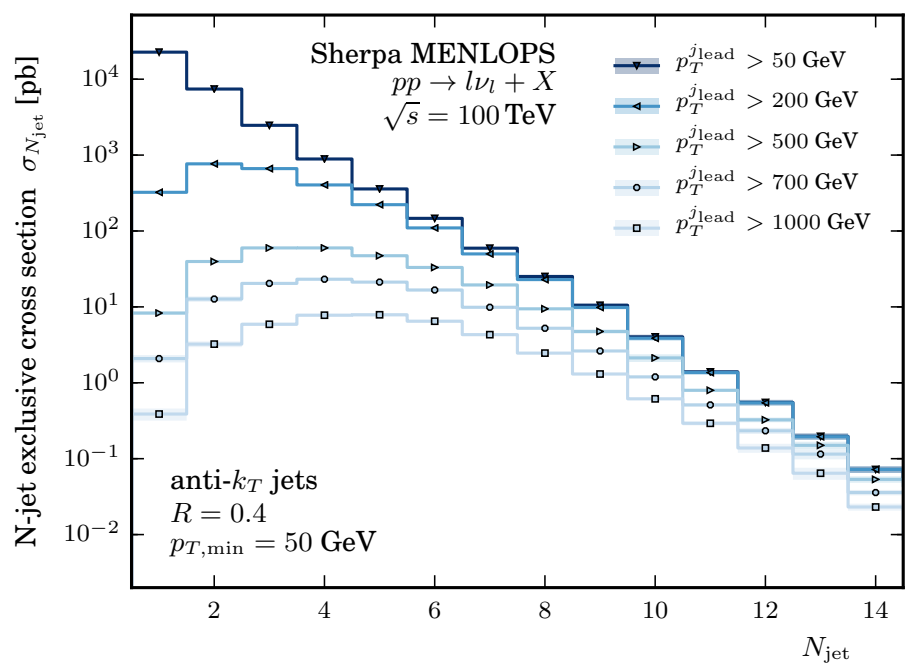

(a) leading jet cut

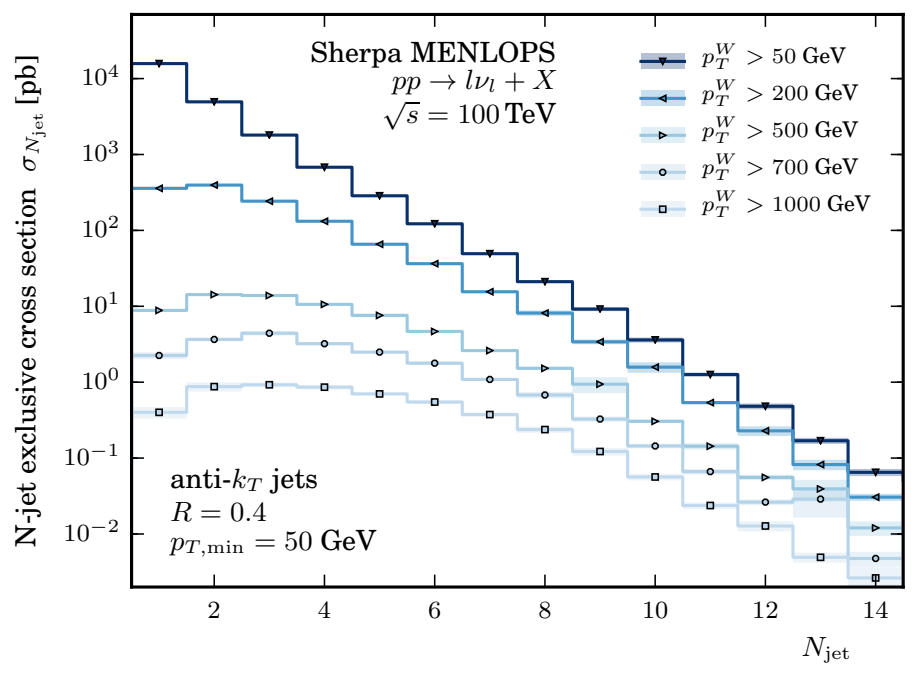

(b) W-boson cut

Figure 5.7.: Results of a MENLOPS calculation for the exclusive jet multiplicity $N_{\text {jet }}$ for off-shell W-boson production in association with jets for different cuts on the leading jet and the W-boson transverse momentum. For all (subsequent) jets a cut of $p_{T}>50 \mathrm{GeV}$ is applied. 


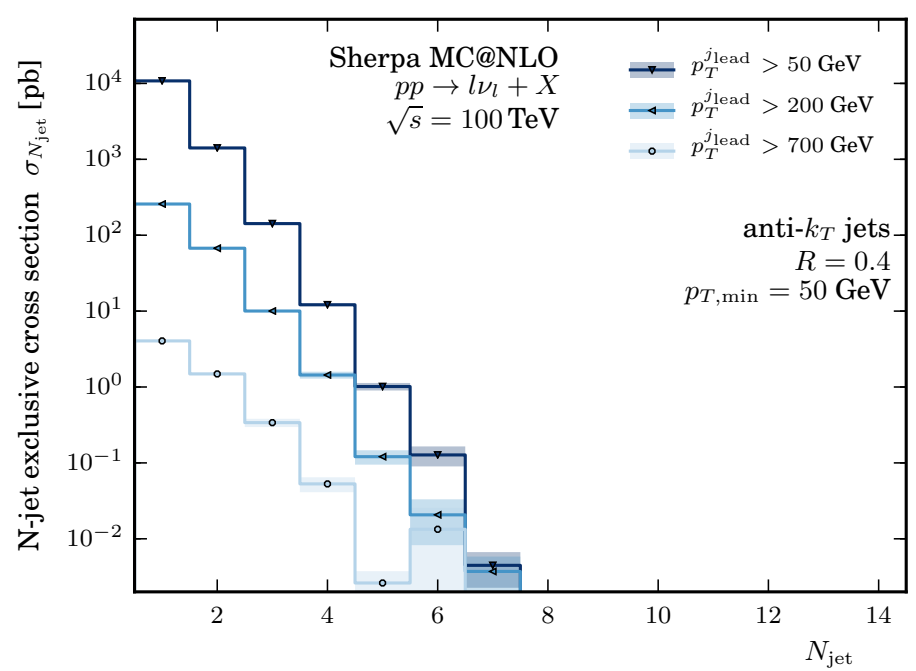

(a) leading jet cut

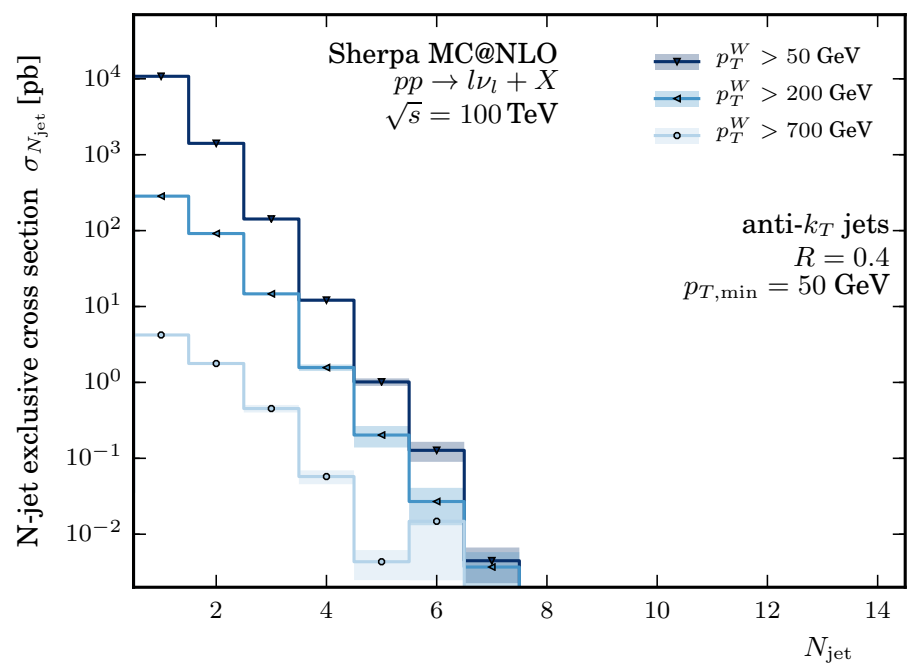

(b) W-boson cut

Figure 5.8.: The same as in Fig. 5.7, but with a MC@NLO calculation instead of a MENLOPS one. 


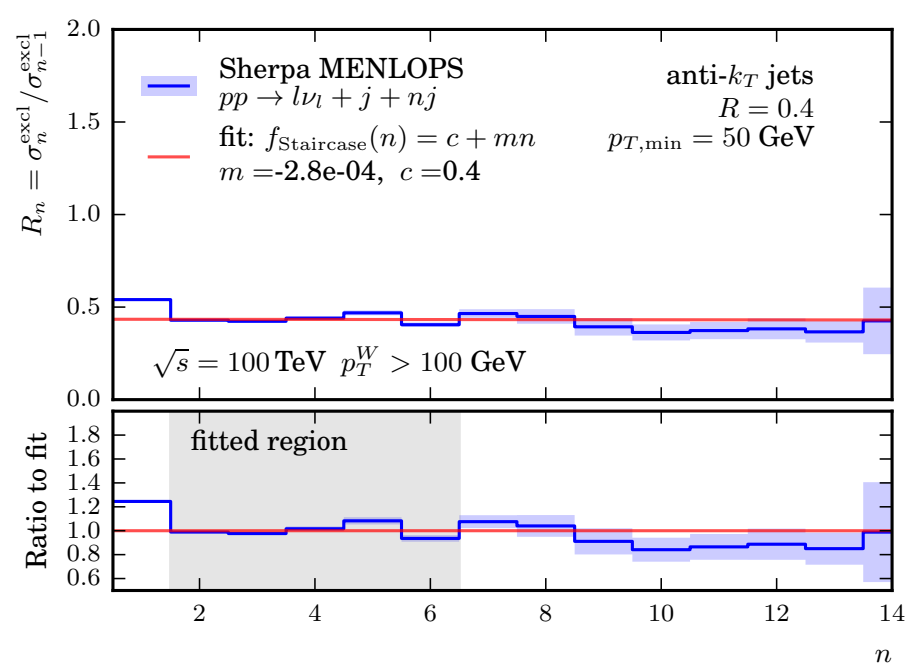

Figure 5.9.: Results of a MENLOPS calculation for exclusive jet multiplicity ratios in off-shell W-boson production in association with jets for a democratic jet selection, i.e. applying a universal jet cut of $p_{T, \text { min }}=50 \mathrm{GeV}$ and requiring $p_{T, W}>100 \mathrm{GeV}$. As the fit function the Staircase hypothesis given in Eq. (5.2) has been used.

the corresponding results for hierarchical selection criteria are presented. Two cut scenarios have been considered, namely $p_{T}^{\text {jead }}>500 \mathrm{GeV}$ and $p_{T}^{\mathrm{W}}>500 \mathrm{GeV}$, while $p_{T, \min }=50 \mathrm{GeV}$ is required otherwise. The results for the fits of the Poisson hypothesis in the range $1 \leq n \leq 4$ illustrate the significantly larger average jet number $\bar{n}=2.7$ in the first case vs. $\bar{n}=1.1$ in the latter case. The constant offset parameters $c$ are determined as $c=0.1$ and $c=0.4$, respectively. The extrapolations of both fits yield a good description of the simulated data up to very high jet counts.

To further illustrate the universality of jet-scaling patterns, Fig. 5.11 compiles the inclusive jet multiplicity ratios for a variety of processes, including pure jets, $\gamma+$ jets, $t \bar{t}+$ jets and $\mathrm{W} / \mathrm{Z}+$ jets. The predictions are based on dedicated $n$-jet tree-level matrix-element calculations, without invoking parton showers. Democratic jet selection cuts are applied, i.e. requiring $p_{T}^{j}>50 \mathrm{GeV}$ in all processes. In addition, the photon production processes are regulated by the selection criteria $p_{T}^{\gamma}>50 \mathrm{GeV}$ and $R_{j, \gamma}>0.4$, with $R_{j, \gamma}$ the $(\eta-\phi)$-distance between any of the jets and the photon.

There are a few remarkable aspects to note here. Apparently, for the pure jets and the $\mathrm{W}+$ jets processes these $\mathrm{LO}$ rate estimates nicely reproduce the staircase scaling parameters found in the matrix-element plus parton-shower samples for the analogous jet-selection cuts, cf. Figs. 5.6a and 5.9. This is supported by the fact that for exact Staircase scaling the cross-section ratios for subsequent jet multiplicities are identical for exclusive and inclusive 


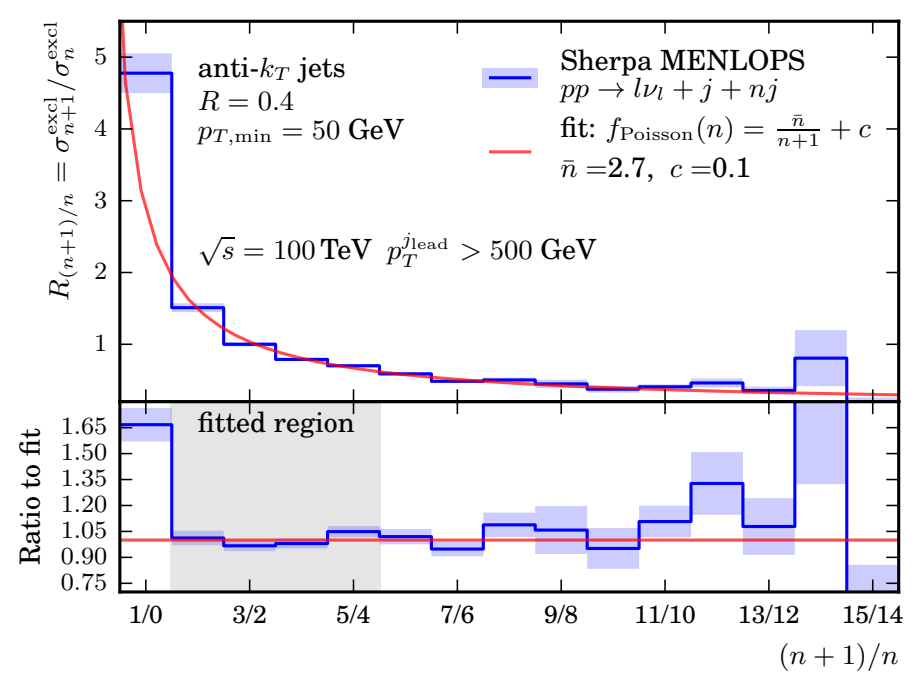

(a) leading jet $p_{T}>500 \mathrm{GeV}$

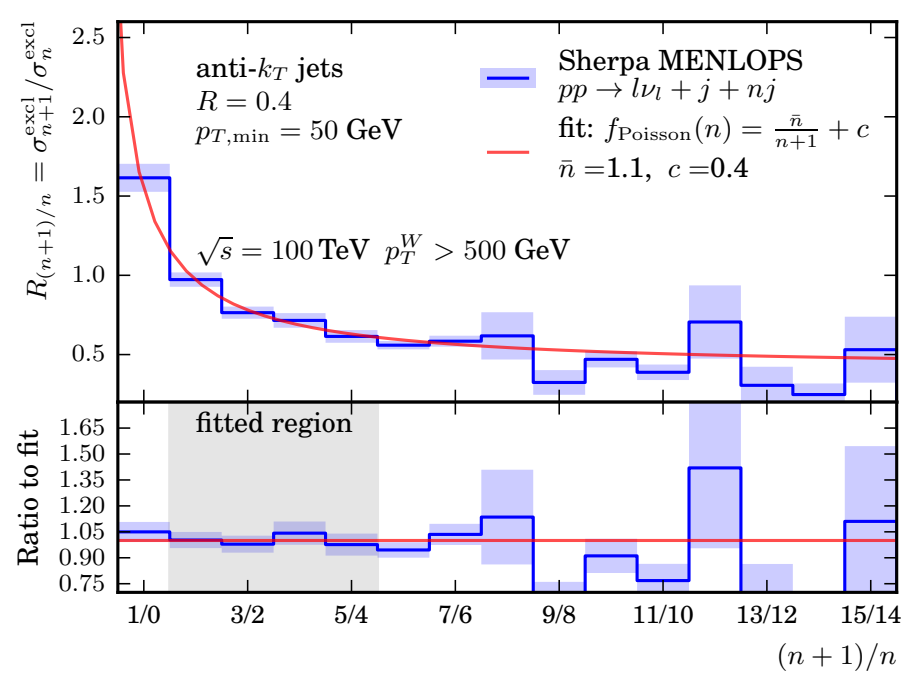

(b) $p_{T}^{\mathrm{w}}>500 \mathrm{GeV}$

Figure 5.10.: The same as in Fig. 5.9, but for hierarchical event selection cuts. For the fit, the Poisson hypothesis given in Eq. (5.2) has been used. 


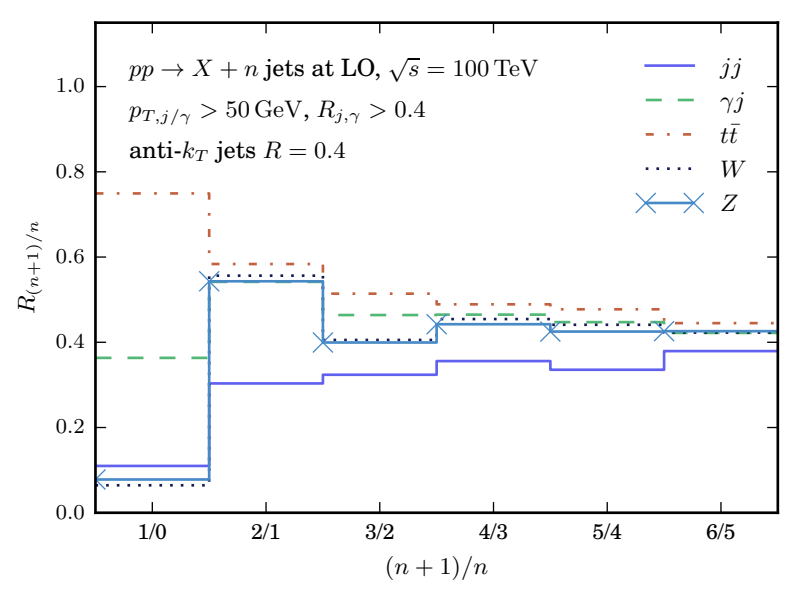

Figure 5.11.: The inclusive jet multiplicity ratio $R_{(n+1) / n}$ for several processes calculated at LO for each final-state multiplicity. Note that the index $n$ counts jets associated to the core process listed in the legend.

cross sections [66], i.e. in this limit

$$
\frac{\sigma_{n+1}^{\text {excl }}}{\sigma_{n}^{\text {excl }}}=\frac{\sigma_{n+1}^{\text {incl }}}{\sigma_{n}^{\text {incl }}}=R_{(n+1) / n}=\text { const. }
$$

Also, the $\mathrm{Z}+\mathrm{jets}$ result resembles the $\mathrm{W}+\mathrm{jets}$ one very closely, and for $\gamma \mathrm{j}+\mathrm{jets}$, the same overall behaviour is found. However, the production of a pair of top quarks induces a large upper scale for subsequent jet emission. Correspondingly, the plateau is more slowly approached from the top, indicating an initially Poisson-like scaling.

\subsection{Discussion}

Moving to a centre-of-mass energy of $100 \mathrm{TeV}$ comes with a large amount of increased complexity and challenges, but also a very large potential for the discovery of New Physics and tests of the current SM. This chapter presented results demonstrating the overwhelming amount of QCD radiation at such a proton-proton collider. Although some techniques used at the LHC can also be used to study a $100 \mathrm{TeV}$ environment, others will need to be adapted or improved.

We demonstrated that one can make use of dominant jet-multiplicity scaling patterns in democratic and hierarchic kinematic regimes at the FCC to extrapolate known fixedorder or Monte-Carlo results to higher multiplicities. These two regimes feature Staircase and Poisson scaling, respectively, and we found good agreement between scaling-based extrapolations and simulation results in both cases. It is possible to fit jet multiplicities $n$ up to values of $n=15$ or even higher, using results for much lower $n$. 
These extrapolation techniques allow trustworthy predictions to be made for very high jet-multiplicity bins that will be populated by a variety of production processes at FCC energies. Hence, the use of jet-scaling patterns can be of use to separate New Physics signals from QCD backgrounds in multi-jet channels, based on the shape of the jet multiplicity distribution. 


\section{Conclusions}

Data sets from the current and future runs at the LHC and proposed colliders will have an unprecedented statistical precision. Recent measurements, e.g. of the Z-boson transverse momentum distribution, foreshadow this precision era, in which theory predictions need to be ready to provide accuracies of only a few percent at most, even for differential observables. However, (N)NLO QCD predictions are computationally expensive, especially when dressed with parton showers and non-perturbative effects using Monte-Carlo event generators. Hence, explicit re-calculations for scale and parameter variations to estimate the theory uncertainties can be prohibitive, in particular for fits of multi-variate objects such as PDFs. But precision measurements and searches for New Physics at high-energy colliders require QCD theory uncertainties to be fully accounted for, and that determinations of parameters such as PDFs or $\alpha_{S}$ continue to improve. Moreover, higher collider energies will lead to large rates of high-multiplicity jet production. Predicting these rates is also computationally challenging. To face these issues, fast and ready-to-use methods to evaluate QCD predictions are necessary.

With this thesis we provided significant new contributions to reweighting approaches, allowing for fast re-evaluations of state-of-the-art perturbative QCD predictions. By presenting new developments for both event-by-event and interpolation-grid reweighting techniques, we gave an exhaustive treatment on the subject. In addition, we studied highmultiplicity jet rates at the FCC and the application of approximate jet-scaling laws for the extrapolation of those rates.

Firstly, we have presented the implementation and validation of event-by-event reweighting techniques allowing for the fast and efficient evaluation of perturbative and parametric systematic uncertainties in the SHERPA event-generator framework. We have lifted the available techniques for the determination of PDF, $\alpha_{S}$ and scale uncertainties in leadingand next-to-leading-order QCD calculations to include the respective variations in partonshower simulations. In turn, we provide the means to perform consistent uncertainty evaluations for multi-jet merged simulations based on leading- or next-to-leading-order accurate matrix elements of varying multiplicity matched with parton showers.

With our extensive validation we have been able to prove the correctness of the implementation and have, furthermore, been able to illustrate the importance of parton-shower reweighting for reliable uncertainty estimates. With comparably little additional computational costs this allows for the on-the-fly determination of PDF, $\alpha_{S}$ and scale uncertainties based on one single generator run, that, otherwise, would require explicit re-computations. The overall reduction in CPU time is by a factor of about 3 to 20 , depending on the eventgeneration mode used. Even larger reduction factors can be achieved when the events are 
further processed through a detector simulation.

Hence, considering its scope and the amount of saved CPU time it offers, the presented reweighting implementation is ideally suited for event-wise uncertainty estimates and can readily be used in arbitrary theoretical and experimental analyses.

Secondly, we have presented new developments for the reweighting through QCD interpolation grids, as implemented in the APPLGRID and FAsTNLO packages. These store the perturbative coefficients for a certain observable calculation discretised in $x$ and $Q^{2}$. Using interpolation methods, this allows for the a posteriori inclusion of PDFs, $\alpha_{S}$ and variations of the renormalisation and factorisation scales. In turn, such techniques are well suited for (combined) fits of PDFs and $\alpha_{S}$ that require a multitude of re-computations of the theoretical predictions. The automated projection of arbitrary NLO QCD calculations onto such grids is done by the recently released AMCFAST and MCGRID tools. We discussed and validated new features in MCGRID. A new interface has been added to support the FASTNLO package. This supplements the already existing interface to APPLGRID, which in turn has been enhanced to allow for more flexible scale variations. This makes use of dedicated scale logarithm grids instead of an RGE approach that is restricted to simple scale factors. The third new MCGRID feature is the support for filling the $\mathcal{O}\left(\alpha_{S}\right)$ expansion of S-MC@NLO calculations. These improvements allow for a more versatile automated interpolation-grid production, supporting a larger class of $\mathrm{pQCD}$ calculations, grid implementations and scale variations.

Lastly, we explored the multi-jet environment of a $100 \mathrm{TeV}$ proton-proton collider, applying a method to extrapolate low-multiplicity jet rates to high-multiplicity ones to overcome computational limits of explicit calculations. This extrapolation rests on universal scaling patterns in these jet rates, namely the Staircase and the Poisson scaling, which are found in two limiting kinematic regimes, respectively. Using SHERPA Monte-Carlo studies for different processes in association with additional jets, we found clean manifestations of both patterns. Using these, we could show that a fit to the first few jet multiplicity rates can be used to extrapolate rates for up to 15 or more jets. Hence, this method can be used to discriminate New Physics signals and QCD backgrounds in the tails of jet-multiplicity distributions.

Throughout the thesis we have seen large deviations between predictions when moving from "simple" (N)LO (plus parton-shower) calculations to more complex ones using multi-jet merging, confirming earlier results. This was e.g. the case for the W-boson $p_{T}$ or the number of jets in $\mathrm{W}$-boson production, both for our $13 \mathrm{TeV}$ and our $100 \mathrm{TeV}$ samples. Also the scale uncertainties generally got smaller, although they can be spuriously small for LO matrix elements. These findings clearly highlight the need to use state-of-the-art MEPS@NLO calculation for those and other observables whenever possible. With this thesis, we provided means to make the application of such calculations less expensive in terms of CPU cycles, and hence more feasible.

To give an outlook, the most obvious improvement for both the internal reweighting in SHERPA and the MCGRID interface would be the support for NNLO calculations. For the 
internal reweighting, this should also include the matching to a parton shower, as presented in $[34,35]$. It would be advantageous to start with the internal reweighting, because this entails tracing the dependences in SHERPA. This would make it easy to output the correct weights and to implement their projection to grid weights in MCGRID afterwards. The motivation for supporting NNLO (+ parton shower) is even stronger than for NLO, due to the significantly higher CPU time requirements. Furthermore, the extension to NNLO QCD is comparatively straightforward. For these reasons, it is planned for the near future.

Another follow-up would be the application of the internal reweighting to an $\alpha_{S}$ fit based on multiple jet observables. A similar study has recently been presented for NLO calculations of Z+jets observables, based on the reweighting of NTUPLE event files [138]. With our internal reweighting, we could make use of an NLO multi-jet merged calculation, which gives a more faithful description of jet distributions.

A milestone for interpolation grids would be the inclusion of parton-shower effects. Alternatively, the support for the matrix-element parts of a multi-jet merged calculation can be attempted. The vantage point of a complete account of an internal reweighting helped us to identify the problems with these two enhancements. Especially the variable number of PDF ratios is difficult to encode on a reasonable number of grids. However, we found indications in our internal-reweighting discussion that not all PDF ratios are equally important and this might point to suitable approximations that help to mould many-/allorder calculations into a form that is compatible with interpolation grids. This would allow for the inclusion of phase-space regions in PDF determinations, that are not well described by fixed-order calculations. 


\section{A. Configuring and accessing event-weight variations}

SHERPA provides a list of pre-calculated alternative event weights, which are automatically output to the HEPMC event record or directly to an interfaced RIVET analysis. For versions of SHERPA later than v.2.2.0, the variations to calculate can be specified with the following line in the (run) section of the SHERPA run card:

VARIATIONS muR2fac1, muF2fac1,PDF1 muR2fac2, muF2fac2,PDF2 ..;

Each variation is characterised by up to three arguments

muR2fac a prefactor multiplying the nominal (squared) renormalisation scale

$\mathrm{muF} 2 \mathrm{fac}$ a prefactor multiplying the nominal (squared) factorisation scale

PDF a parton density and its accompanying $\alpha_{S}$ parametrisation.

This syntax works for all employed scale setters of SHERPA and both SHERPA's internal PDFs and PDFs interfaced through LHAPDF (v. 5 or later). If trailing arguments are omitted from a variation, their default values are used, which is 1.0 for scale factors and the PDF set used by SHERPA for the nominal calculation.

In He PMC event records (v. 2.06 or later), the alternate weights can be accessed as named weights within the HepMC: : WeightContainer of each event. The keys are given in one of the following formats:

MUR $<$ muR2fac $>$ _MUF $<$ muF2fac $>$ _PDF $<$ ID $>$

MUR $<$ muR2fac $>$ _MUF $<$ muF 2 fac $>$ _PDF $<$ ID $>$ _PSMUR $<$ muR2fac $>$ _PSMUF $<m u F 2$ fac $>$

The parts in angle brackets are replaced with the respective scale factors and LHAPDFIDs. The second form is used, if a factor is applied to the renormalisation/factorisation scale of parton-shower emissions. This includes branchings within cluster histories determined by the multi-jet merging procedure, as discussed in Section 3.7. If the scale reweighting with parton-shower emissions has been enabled (we discuss below how to do so), the scale factors for MUR, MUF and PSMUR, PSMUF are always equal, respectively, in the current implementation.

If the internal RIVET interface of SHERPA is used to analyse events during the generation, one histogram file per variation is written to disk, along with the nominal one. The file names follow a pattern resembling the HePMC weight-container keys as specified above. 


\section{Scale variations}

The scale argument can also be specified by enclosing it in square brackets: [mu2fac]. This syntactic sugar implies both the given factor, its inverse and the default value. For example, $1.0,[4.0]$ is equivalent to $1.0,4.01 .0,0.251 .0,1.0$ and therefore triggers up and down variations of the factorisation scale, along with the central value. If both scale factors are enclosed in brackets, they are expanded individually, keeping the other at its default value of 1.0: Hence, [4.0] , [4.0] is equivalent to the 5-point scale variation 4.0,1.0 0.25,1.0 1.0,4.0 1.0,0.25 1.0,1.0. To include simultaneous variations in the same direction, both factors can be surrounded by a single pair of brackets. Thus, $[4.0,4.0]$ is equivalent to the 7-point scale variation 4.0,1.0 0.25,1.0 1.0,4.0 1.0,0.25 4.0,4.0 $0.25,0.251 .0,1.0$.

\section{PDF and $\alpha_{S}$ variations}

PDF and $\alpha_{S}$ variations both work by specifying a PDF set through the PDF argument of a variation. This is because SHERPA per default uses the value for $\alpha_{S}\left(m_{\mathrm{Z}}^{2}\right)$ given by the PDF set in use. Therefore an $\alpha_{S}$ variation can be achieved by using PDF fits for different values of $\alpha_{S}\left(m_{\mathrm{Z}}^{2}\right)$.

To specify a specific member of a PDF set, its number is given as an additional argument separated by a slash. Thus, 1.0,1.0, CT14nlo/38 asks for the 38th member of the CT14NLO PDF set, without modifying the renormalisation and factorisation scales. If the slash and the number are not given, the central PDF member is used, i.e. CT14nlo is equivalent to CT14nlo/0.

SHERPA can also be asked to do variations for all members of a PDF set by enclosing it in square brackets. Hence, 1.0,1.0, [CT14nlo] is equivalent to

$$
\text { 1.0,1.0,CT14nlo/0 1.0,1.0, CT14nlo/1 ‥ 1.0,1.0, CT14nlo/56 }
$$

This $[\mathrm{PDF}]$-notation only works with PDFs interfaced through LHAPDF v. 6. It can be combined with scale factors that are enclosed in square brackets. Again, the expansions are done individually, keeping other arguments at their default values. This means that for example 1.0, [4.0], [CT14nlo] is equivalent to 1.0, [4.0] 1.0,1.0, [CT14nlo]. Hence, a 7-point scale variation and a full CT14nlo PDF variation can be requested by

VARIATIONS $[4.0,4.0],[\mathrm{CT} 14 \mathrm{nl} \mathrm{O}]$;

\section{Configuring how variations are calculated}

The following options always affect all variations that are specified by arguments to the VARIATIONS keyword.

REWEIGHT_SPLITTING_ALPHAS_SCALES (default: o) If this is set to 1 , the renormalisation scale factor is applied to the $\alpha_{S}$ argument of individual splittings, instead of applying it 
only to the overall renormalisation scale, see Section 3.7. This means that parton-shower emissions are only included in the rescaling, if this option is enabled. In the notation of Sections 3.3 and 3.7 , this sets $\tilde{k}_{\alpha_{S}}=\tilde{\mu}_{R} / \mu_{R}$.

REWEIGHT_SPLITTING_PDF_SCALES (default: o) If this is set to 1 , the factorisation scale factor is also applied to PDF scale arguments within shower emissions (and intermediate cluster history PDF ratios), and not only to the core-process PDFs. In the notation of Sections 3.3 and 3.7 , this sets $\tilde{k}_{f}=\tilde{\mu}_{F} / \mu_{F}$.

REWEIGHT_MAXEM (default: -1) This option specifies the number of ordinary parton-shower emissions included in the reweighting per event. If this is set to o, no emission is reweighted. The default value -1 means that all emissions should be reweighted.

REWEIGHT_MCATNLO_EM (default: 1) If this is set to o, the single parton-shower emission within the S-MC@NLO contribution is not reweighted.

VARIATIONS_INCLUDE_CV (default: 1 ) If this is set to o, the behaviour of the square bracket syntax is changed, such that the central-value variation is not included when expanding a parameter in square brackets. It is recommended not to disable it, such that one can do a closure test between the dedicated calculation and the reweighting. However, in CPU intensive applications, this setting can be used to omit this one obsolete variation while still making use of the convenient square-bracket syntax. 


\section{Bibliography}

[1] G. Aad et al. (ATLAS), Observation of a new particle in the search for the Standard Model Higgs boson with the ATLAS detector at the LHC, Phys. Lett. B716 (2012), 1

[2] S. Chatrchyan et al. (CMS), Observation of a new boson at a mass of $125 \mathrm{GeV}$ with the CMS experiment at the LHC, Phys. Lett. B716 (2012), 30

[3] S. L. Glashow, Partial Symmetries of Weak Interactions, Nucl. Phys. 22 (1961), 579; S. L. Glashow, J. Iliopoulos, and L. Maiani, Weak Interactions with Lepton-Hadron Symmetry, Phys. Rev. D2 (1970), 1285; S. Weinberg, A Model of Leptons, Phys. Rev. Lett. 19 (1967), 1264; H. Georgi and S. L. Glashow, Unified weak and electromagnetic interactions without neutral currents, Phys. Rev. Lett. 28 (1972), 1494; D. J. Gross and F. Wilczek, Ultraviolet Behavior of Nonabelian Gauge Theories, Phys. Rev. Lett. 30 (1973), 1343; H. D. Politzer, Reliable Perturbative Results for Strong Interactions?, Phys. Rev. Lett. 30 (1973), 1346; H. D. Politzer, Asymptotic Freedom: An Approach to Strong Interactions, Phys. Rept. 14 (1974), 129; A. Salam, Weak and Electromagnetic Interactions, Conf. Proc. C680519 (1968), 367

[4] G. Apollinari et al., High-Luminosity Large Hadron Collider (HL-LHC): Preliminary Design Report, Geneva: CERN, 2015

[5] E. Eichten et al., Super Collider Physics, Rev. Mod. Phys. 56 (1984), 579, [Addendum: Rev. Mod. Phys. 58 (1986), 1065]

[6] N. Arkani-Hamed et al., Physics Opportunities of a $100 \mathrm{TeV}$ Proton-Proton Collider, Phys. Rept. 652 (2016), 1

[7] A. Avetisyan et al., Methods and Results for Standard Model Event Generation at $\sqrt{s}$ $=14 \mathrm{TeV}, 33 \mathrm{TeV}$ and $100 \mathrm{TeV}$ Proton Colliders (A Snowmass Whitepaper), Proceedings, Community Summer Study 2013: Snowmass on the Mississippi (CSS2013): Minneapolis, MN, USA, July 29-August 6, 2013

[8] I. Hinchliffe et al., Luminosity goals for a 10o-TeV pp collider, Int. J. Mod. Phys. A30 (2015), 1544002

[9] A. Nisati, Prospects On Standard Model And Higgs Physics At The HL-LHC, tech. rep., Geneva: CERN, 2016

[10] J. K. Behr et al., Boosting Higgs pair production in the $b \bar{b} b \bar{b}$ final state with multivariate techniques, Eur. Phys. J. C76 (2016), 386 
[11] G. Aad et al. (ATLAS), Measurement of the transverse momentum and $\phi_{\eta}^{*}$ distributions of Drell-Yan lepton pairs in proton-proton collisions at $\sqrt{s}=8 \mathrm{TeV}$ with the ATLAS detector, Eur. Phys. J. C76 (2016), 291

[12] J. M. Campbell et al., Predictions for diphoton production at the LHC through NNLO in QCD, JHEP o7 (2016), 148

[13] E. L. Berger et al., NNLO QCD Corrections to t-channel Single Top-Quark Production and Decay, (2016), arXiv: 1606.08463 [hep-ph]

[14] M. Czakon, D. Heymes, and A. Mitov, High-precision differential predictions for top-quark pairs at the LHC, Phys. Rev. Lett. 116 (2016), 082003

[15] M. Grazzini et al., $W^{+} W^{-}$production at the LHC: fiducial cross sections and distributions in NNLO QCD, JHEP o8 (2016), 140

[16] R. Boughezal et al., Z-boson production in association with a jet at next-to-next-toleading order in perturbative QCD, Phys. Rev. Lett. 116 (2016), 152001

[17] R. Boughezal, X. Liu, and F. Petriello, W-boson plus jet differential distributions at NNLO in QCD, (2016), arXiv: 1602.06965 [hep-ph]

[18] A. Gehrmann-De Ridder et al., The NNLO QCD corrections to Z boson production at large transverse momentum, JHEP o7 (2016), 133

[19] R. Boughezal, X. Liu, and F. Petriello, Phenomenology of the Z-boson plus jet process at NNLO, (2016), arXiv: 1602.08140 [hep-ph]

[20] B. I. Ermolaev and V. S. Fadin, Log-Log Asymptotic Form of Exclusive Cross-Sections in Quantum Chromodynamics, JETP Lett. 33 (1981), 269, [Pisma Zh. Eksp. Teor. Fiz. 33 (1981), 285]; A. H. Mueller, On the Multiplicity of Hadrons in QCD Jets, Phys. Lett. B104 (1981), 161; Y. L. Dokshitzer, V. S. Fadin, and V. A. Khoze, On the Sensitivity of the Inclusive Distributions in Parton Jets to the Coherence Effects in QCD Gluon Cascades, Z. Phys. C18 (1983), 37; Y. L. Dokshitzer et al., QCD Coherence in HighEnergy Reactions, Rev. Mod. Phys. 60 (1988), 373

[21] S. Schumann and F. Krauss, A Parton shower algorithm based on Catani-Seymour dipole factorisation, JHEP 03 (2008), 038

[22] Z. Nagy and D. E. Soper, Parton showers with quantum interference, JHEP o9 (2007), 114

[23] M. Dinsdale, M. Ternick, and S. Weinzierl, Parton showers from the dipole formalism, Phys. Rev. D76 (2007), 094003

[24] S. Frixione and B. R. Webber, Matching NLO QCD computations and parton shower simulations, JHEP 06 (2002), 029

[25] P. Nason, A New method for combining NLO QCD with shower Monte Carlo algorithms, JHEP 11 (2004), 040; S. Frixione, P. Nason, and C. Oleari, Matching NLO QCD computations with Parton Shower simulations: the POWHEG method, JHEP 11 (2007), 070 
[26] S. Catani et al., QCD matrix elements + parton showers, JHEP 11 (2001), 063

[27] L. Lonnblad, Correcting the color dipole cascade model with fixed order matrix elements, JHEP 05 (2002), 046

[28] M. L. Mangano, M. Moretti, and R. Pittau, Multijet matrix elements and shower evolution in hadronic collisions: $W b \bar{b}+n$ jets as a case study, Nucl. Phys. B632 (2002), 343

[29] F. Krauss, Matrix elements and parton showers in hadronic interactions, JHEP o8 (2002), 015

[30] S. Höche et al., QCD matrix elements and truncated showers, JHEP 05 (2009), 053

[31] K. Hamilton, P. Richardson, and J. Tully, A Modified CKKW matrix element merging approach to angular-ordered parton showers, JHEP 11 (2009), 038

[32] L. Lonnblad and S. Prestel, Matching Tree-Level Matrix Elements with Interleaved Showers, JHEP O3 (2012), 019

[33] S. Höche et al., QCD matrix elements + parton showers: The NLO case, JHEP o4 (2013), 027

[34] S. Höche, Y. Li, and S. Prestel, Higgs-boson production through gluon fusion at NNLO QCD with parton showers, Phys. Rev. D9o (2014), 054011

[35] S. Höche, Y. Li, and S. Prestel, Drell-Yan lepton pair production at NNLO QCD with parton showers, Phys. Rev. D91 (2015), 074015

[36] S. Höche, Y. Li, and S. Prestel, Combining parton showers and NNLO matrix elements, Proceedings, 5oth Rencontres de Moriond, QCD and high energy interactions: La Thuile, Italy, March 21-28, 2015, 135

[37] K. Hamilton et al., NNLOPS simulation of Higgs boson production, JHEP 10 (2013), 222

[38] A. Karlberg, E. Re, and G. Zanderighi, NNLOPS accurate Drell-Yan production, JHEP o9 (2014), 134

[39] K. Hamilton, P. Nason, and G. Zanderighi, Finite quark-mass effects in the NNLOPS POWHEG+MiNLO Higgs generator, JHEP 05 (2015), 140

[40] M. Schönherr et al., NLO QCD+EW for V+jets, 4th Large Hadron Collider Physics Conference (LHCP 2016) Lund, Sweden, June 13-18, 2016

[41] P. Hagler, Hadron structure from lattice quantum chromodynamics, Phys. Rept. 490 (2010), 49

[42] F. Cascioli et al., Next-to-leading order simulations with Sherpa+OpenLoops, PoS LL2014 (2014), 022

[43] F. Cascioli et al., Precise Higgs-background predictions: merging NLO QCD and squared quark-loop corrections to four-lepton +o,1 jet production, JHEP O1 (2014), 046 
[44] G. Aad et al. (ATLAS), Study of jets produced in association with a $W$ boson in $p p$ collisions at $\sqrt{s}=7 \mathrm{TeV}$ with the ATLAS detector, Phys. Rev. D85 (2012), 092002

[45] Z. Bern et al., Ntuples for NLO Events at Hadron Colliders, Comput. Phys. Commun. 185 (2014), 1443

[46] T. Gleisberg et al., SHERPA 1. alpha: A Proof of concept version, JHEP 02 (2004), 056

[47] T. Gleisberg et al., Event generation with SHERPA 1.1, JHEP 02 (2009), 007

[48] J. Bellm et al., Herwig 7.o/Herwig++ 3.0 release note, Eur. Phys. J. C76 (2016), 196

[49] M. Bähr et al., Herwig++ Physics and Manual, Eur. Phys. J. C58 (2008), 639

[50] T. Sjöstrand et al., An Introduction to PYTHIA 8.2, Comput. Phys. Commun. 191 (2015), 159

[51] T. Carli et al., A posteriori inclusion of parton density functions in NLO QCD finalstate calculations at hadron colliders: The APPLGRID Project, Eur. Phys. J. C66 (2010), 503

[52] T. Kluge, K. Rabbertz, and M. Wobisch, FastNLO: Fast $p Q C D$ calculations for PDF fits, Deep inelastic scattering. Proceedings, 14th International Workshop, DIS 2006, Tsukuba, Japan, April 20-24, 2006, 483

[53] D. Britzger et al., New features in version 2 of the fastNLO project, Proceedings, 2oth International Workshop on Deep-Inelastic Scattering and Related Subjects (DIS 2012), 217

[54] L. Del Debbio, N. P. Hartland, and S. Schumann, MCgrid: projecting cross section calculations on grids, Comput. Phys. Commun. 185 (2014), 2115

[55] E. Bothmann, N. Hartland, and S. Schumann, Introducing MCgrid 2.o: Projecting cross section calculations on grids, Comput. Phys. Commun. 196 (2015), 617

[56] E. Bothmann, M. Schönherr, and S. Schumann, Fast evaluation of theoretical uncertainties with Sherpa and MCgrid, PoS DIS2015 (2015), 136

[57] V. Bertone et al., aMCfast: automation of fast NLO computations for PDF fits, JHEP 08 (2014), 166

[58] G. Aad et al. (ATLAS), Measurement of multi-jet cross sections in proton-proton collisions at a $7 \mathrm{TeV}$ center-of-mass energy, Eur. Phys. J. C71 (2011), 1763

[59] G. Aad et al. (ATLAS), Measurement of the production cross section of jets in association with a $Z$ boson in pp collisions at $\sqrt{s}=7 \mathrm{TeV}$ with the ATLAS detector, JHEP o7 (2013), 032

[6o] G. Aad et al. (ATLAS), Measurements of the W production cross sections in association with jets with the ATLAS detector, Eur. Phys. J. C75 (2015), 82

[61] V. Khachatryan et al. (CMS), Differential cross section measurements for the production of a W boson in association with jets in proton-proton collisions at $\sqrt{s}=7 \mathrm{TeV}$, Phys. Lett. B741 (2015), 12 
[62] C. Englert et al., Jets plus Missing Energy with an Autofocus, Phys. Rev. D83 (2011), 095009

[63] C. Englert et al., Establishing Jet Scaling Patterns with a Photon, JHEP O2 (2012), 030

[64] Z. Bern et al., Extrapolating W-Associated Jet-Production Ratios at the LHC, Phys. Rev. D92 (2015), 014008

[65] E. Gerwick et al., QCD Jet Rates with the Inclusive Generalized kt Algorithms, JHEP 04 (2013), 089

[66] E. Gerwick et al., Scaling Patterns for QCD Jets, JHEP 10 (2012), 162

[67] E. Gerwick and P. Schichtel, Jet properties at high-multiplicity, (2014), arXiv: 1412. 1806 [hep-ph]

[68] M. E. Peskin and D. V. Schroeder, An Introduction to quantum field theory, (1995)

[69] S. Höche and S. Prestel, The midpoint between dipole and parton showers, Eur. Phys. J. C75 (2015), 461

[7o] T. Gleisberg and S. Höche, Comix, a new matrix element generator, JHEP 12 (2008), 039

[71] S. Höche et al., Beyond Standard Model calculations with Sherpa, Eur. Phys. J. C75 (2015), 135

[72] F. Krauss, R. Kuhn, and G. Soff, $A M E G I C++$ 1.o: A Matrix element generator in C++, JHEP O2 (2002), 044

[73] R. Kleiss and W. J. Stirling, Spinor Techniques for Calculating $p$ anti-p $\rightarrow W+-/ Z o$ + Jets, Nucl. Phys. B262 (1985), 235

[74] A. Ballestrero, E. Maina, and S. Moretti, Heavy quarks and leptons at e+e-colliders, Nucl. Phys. B415 (1994), 265

[75] T. Gleisberg et al., Cross-sections for multiparticle final states at a linear collider, Eur. Phys. J. C34 (2004), 173

[76] S. Catani and M. H. Seymour, A General algorithm for calculating jet cross-sections in NLO QCD, Nucl. Phys. B485 (1997), 291, [Erratum: Nucl. Phys. B510 (1998), 503]; S. Catani et al., The Dipole formalism for next-to-leading order QCD calculations with massive partons, Nucl. Phys. B627 (2002), 189

[77] C. F. Berger et al., An Automated Implementation of On-Shell Methods for One-Loop Amplitudes, Phys. Rev. D78 (2008), 036003

[78] F. Cascioli, P. Maierhofer, and S. Pozzorini, Scattering Amplitudes with Open Loops, Phys. Rev. Lett. 108 (2012), 111601

[79] The OpenLoops one-loop generator by F. Cascioli, J. Lindert, P. Maierhöfer and S. Pozzorini is publicly available at http://openloops . hepforge org.

[80] G. Cullen et al., GOSAM-2.o: a tool for automated one-loop calculations within the Standard Model and beyond, Eur. Phys. J. C74 (2014), 3001 
[81] C. Duhr, S. Höche, and F. Maltoni, Color-dressed recursive relations for multi-parton amplitudes, JHEP 08 (2006), 062

[82] G. P. Lepage, Vegas: An Adaptive Multidimensional Integration Program, CLNS$80 / 447,1980$

[83] R. Kleiss, W. J. Stirling, and S. D. Ellis, A New Monte Carlo Treatment of Multiparticle Phase Space at High-energies, Comput. Phys. Commun. 40 (1986), 359

[84] A. van Hameren and C. G. Papadopoulos, A Hierarchical phase space generator for QCD antenna structures, Eur. Phys. J. C25 (2002), 563

[85] Z. Nagy, Matching parton showers to NLO computation, Proceedings, 4oth Rencontres de Moriond on QCD and High Energy Hadronic Interactions: La Thuile, Aosta Valley, Italy, March 12-19, 2005, 201

[86] Z. Nagy and D. E. Soper, A New parton shower algorithm: Shower evolution, matching at leading and next-to-leading order level, Proceedings, Ringberg Workshop on New Trends in HERA Physics 2005, 101

[87] Höche, Stefan and Krauss, Frank and Schönherr, Marek and Siegert, Frank, A critical appraisal of NLO+PS matching methods, JHEP o9 (2012), 049

[88] T. Gehrmann et al., NLO QCD matrix elements + parton showers in $e^{+} e^{-} \rightarrow$ hadrons, JHEP O1 (2013), 144

[89] M. Schonherr and F. Krauss, Soft Photon Radiation in Particle Decays in SHERPA, JHEP 12 (2008), 018

[9o] D. R. Yennie, S. C. Frautschi, and H. Suura, The infrared divergence phenomena and high-energy processes, Annals Phys. 13 (1961), 379

[91] T. Sjostrand and M. van Zijl, A Multiple Interaction Model for the Event Structure in Hadron Collisions, Phys. Rev. D36 (1987), 2019

[92] T. D. Gottschalk, A Realistic Model for e+e-Annihilation Including Parton Bremsstrahlung Effects, Nucl. Phys. B214 (1983), 201; T. D. Gottschalk, An Improved Description of Hadronization in the QCD Cluster Model for $e^{+} e^{-}$Annihilation, Nucl. Phys. B239 (1984), 349; B. R. Webber, A QCD Model for Jet Fragmentation Including Soft Gluon Interference, Nucl. Phys. B238 (1984), 492; T. D. Gottschalk and D. A. Morris, A New Model for Hadronization and $e^{+} e^{-}$Annihilation, Nucl. Phys. B288 (1987), 729

[93] J.-C. Winter, F. Krauss, and G. Soff, A Modified cluster hadronization model, Eur. Phys. J. C36 (2004), 381

[94] J. H. Kuhn and A. Santamaria, Tau decays to pions, Z. Phys. C48 (1990), 445

[95] G. Ecker et al., The Role of Resonances in Chiral Perturbation Theory, Nucl. Phys. B321 (1989), 311 
[96] I. I. Balitsky, V. M. Braun, and A. V. Kolesnichenko, Radiative Decay Sigma+ $\rightarrow>p$ gamma in Quantum Chromodynamics, Nucl. Phys. B312 (1989), 509; V. M. Braun and I. E. Filyanov, QCD Sum Rules in Exclusive Kinematics and Pion Wave Function, Z. Phys. C44 (1989), 157, [Yad. Fiz. 50 (1989), 818]; V. L. Chernyak and I. R. Zhitnitsky, B meson exclusive decays into baryons, Nucl. Phys. B345 (1990), 137

[97] M. R. Whalley, D. Bourilkov, and R. C. Group, The Les Houches accord PDFs (LHAPDF) and LHAGLUE, HERA and the LHC: A Workshop on the implications of HERA for LHC physics. Proceedings, Part B, 2005

[98] A. Buckley et al., LHAPDF6: parton density access in the LHC precision era, Eur. Phys. J. C75 (2015), 132

[99] R. D. Ball et al. (NNPDF), Parton distributions for the LHC Run II, JHEP O4 (2015), 040

[10o] H.-L. Lai et al., New parton distributions for collider physics, Phys.Rev. D82 (2010), 074024

[101] S. Dulat et al., New parton distribution functions from a global analysis of quantum chromodynamics, Phys. Rev. D93 (2016), 033006

[102] A. D. Martin et al., Parton distributions for the LHC, Eur. Phys. J. C63 (2009), 189

[103] L. A. Harland-Lang et al., Parton distributions in the LHC era: MMHT 2014 PDFs, Eur. Phys. J. C75 (2015), 204

[104] J. Pumplin et al., Uncertainties of predictions from parton distribution functions. 2. The Hessian method, Phys. Rev. D65 (2001), 014013

[105] M. Dobbs and J. B. Hansen, The HepMC C++ Monte Carlo event record for High Energy Physics, Comput. Phys. Commun. 134 (2001), 41

[106] A. Buckley et al., Rivet user manual, Comput. Phys. Commun. 184 (2013), 2803

[107] M. Cacciari, G. P. Salam, and G. Soyez, The Anti-k(t) jet clustering algorithm, JHEP 04 (2008), 063

[108] S. Catani et al., New clustering algorithm for multi - jet cross-sections in e+e-annihilation, Phys. Lett. B269 (1991), 432

[109] M. Cacciari, G. P. Salam, and G. Soyez, FastJet User Manual, Eur. Phys. J. C72 (2012), 1896

[110] A. Denner et al., Predictions for all processes $e+e-\longrightarrow 4$ fermions + gamma, Nucl. Phys. B560 (1999), 33; A. Denner and S. Dittmaier, The Complex-mass scheme for perturbative calculations with unstable particles, Nucl. Phys. Proc. Suppl. 160 (2006), 22

[111] N. Cabibbo, Unitary Symmetry and Leptonic Decays, Phys. Rev. Lett. 10 (1963), 531; M. Kobayashi and T. Maskawa, CP Violation in the Renormalizable Theory of Weak Interaction, Prog. Theor. Phys. 49 (1973), 652 
[112] S. Frixione, Z. Kunszt, and A. Signer, Three jet cross-sections to next-to-leading order, Nucl. Phys. B467 (1996), 399

[113] G. Gustafson and U. Pettersson, Dipole Formulation of QCD Cascades, Nucl. Phys. B306 (1988), 746

[114] T. Sjostrand and P. Z. Skands, Transverse-momentum-ordered showers and interleaved multiple interactions, Eur. Phys. J. C39 (2005), 129

[115] J.-C. Winter and F. Krauss, Initial-state showering based on colour dipoles connected to incoming parton lines, JHEP o7 (2008), 040

[116] W. T. Giele, D. A. Kosower, and P.Z. Skands, A simple shower and matching algorithm, Phys. Rev. D78 (2008), 014026

[117] D. Amati et al., A Treatment of Hard Processes Sensitive to the Infrared Structure of QCD, Nucl. Phys. B173 (1980), 429

[118] S. Chatrchyan et al. (CMS), Measurement of differential cross sections for the production of a pair of isolated photons in pp collisions at $\sqrt{s}=7$ TeV, Eur. Phys. J. C74 (2014), 3129

[119] M. Aaboud et al. (ATLAS), Measurement of the $W^{ \pm} Z$ boson pair-production cross section in pp collisions at $\sqrt{s}=13 \mathrm{TeV}$ with the ATLAS Detector, Phys. Lett. B759 (2016), 601

[120] S. Alioli et al., NLO Higgs boson production via gluon fusion matched with shower in POWHEG, JHEP O4 (2009), 002

[121] J. R. Andersen et al., Les Houches 2013: Physics at TeV Colliders: Standard Model Working Group Report, (2014), arXiv: 1405.1067 [hep-ph]

[122] N. Lavesson and L. Lonnblad, W+jets matrix elements and the dipole cascade, JHEP 07 (2005), 054

[123] R. Frederix and S. Frixione, Merging meets matching in MC@NLO, JHEP 12 (2012), 061

[124] K. Hamilton and P. Nason, Improving NLO-parton shower matched simulations with higher order matrix elements, JHEP 06 (2010), 039

[125] S. Höche et al., NLO matrix elements and truncated showers, JHEP o8 (2011), 123

[126] S. Höche et al., Triple vector boson production through Higgs-Strahlung with NLO multijet merging, Phys. Rev. D89 (2014), 093015

[127] Z. Bern et al., Next-to-Leading Order W + 5-Jet Production at the LHC, Phys. Rev. D88 (2013), 014025

[128] J. R. Andersen et al., Les Houches 2015: Physics at TeV Colliders Standard Model Working Group Report, 9 th Les Houches Workshop on Physics at TeV Colliders (PhysTeV 2015) Les Houches, France, June 1-19, 2015 
[129] C. Anastasiou et al., Higgs Boson Gluon-Fusion Production in QCD at Three Loops, Phys. Rev. Lett. 114 (2015), 212001

[130] J. Bellm et al., Parton Shower Uncertainties with Herwig 7: Benchmarks at Leading Order, (2016), arXiv: 1605.01338 [hep-ph]

[131] S. Gieseke, Uncertainties of Sudakov form-factors, JHEP O1 (2005), 058

[132] A. Buckley, Sensitivities to PDFs in parton shower MC generator reweighting and tuning, (2016), arXiv: 1601.04229 [hep-ph]

[133] J. Butterworth et al., PDF4LHC recommendations for LHC Run II, J. Phys. G43 (2016), 023001

[134] A. Buckley et al., General-purpose event generators for LHC physics, Phys. Rept. 504 (2011), 145

[135] A. Buckley et al., Systematic event generator tuning for the LHC, Eur. Phys. J. C65 (2010), 331

[136] P. Z. Skands, Tuning Monte Carlo Generators: The Perugia Tunes, Phys. Rev. D82 (2010), 074018

[137] R. Frederix et al., Four-lepton production at hadron colliders: aMC@NLO predictions with theoretical uncertainties, JHEP 02 (2012), 099

[138] D. Maitre, G. Heinrich, and M. Johnson, N(N)LO event files: applications and prospects, 13th DESY Workshop on Elementary Particle Physics: Loops and Legs in Quantum Field Theory (LL2016) Leipzig, Germany, April 24-29, 2016

[139] J. Bellm et al., Reweighting Parton Showers, Phys. Rev. D94 (2016), 034028

[140] S. Mrenna and P. Skands, Automated Parton-Shower Variations in Pythia 8, Submitted to: Phys. Rev. D (2016)

[141] W. T. Giele, D. A. Kosower, and P. Z. Skands, Higher-Order Corrections to Timelike Jets, Phys. Rev. D84 (2011), 054003

[142] E. Bothmann, M. Schönherr, and S. Schumann, Reweighting QCD matrix-element and parton-shower calculations, Submitted to: Eur. Phys. J. (2016)

[143] T. Gleisberg and F. Krauss, Automating dipole subtraction for QCD NLO calculations, Eur. Phys. J. C53 (2008), 501

[144] S. Badger et al., Numerical evaluation of virtual corrections to multi-jet production in massless QCD, Comput. Phys. Commun. 184 (2013), 1981

[145] T. Binoth et al., A Proposal for a standard interface between Monte Carlo tools and one-loop programs, Comput. Phys. Commun. 181 (2010), 1612, [,1(2010)]

[146] C. F. Berger et al., Precise Predictions for W +4 Jet Production at the Large Hadron Collider, Phys. Rev. Lett. 106 (2011), 092001 
[147] Y. L. Dokshitzer, Calculation of the Structure Functions for Deep Inelastic Scattering and e+e-Annihilation by Perturbation Theory in Quantum Chromodynamics. Sov. Phys. JETP 46 (1977), 641, [Zh. Eksp. Teor. Fiz. 73 (1977), 1216]; G. Altarelli and G. Parisi, Asymptotic Freedom in Parton Language, Nucl. Phys. B126 (1977), 298; V. N. Gribov and L. N. Lipatov, Deep inelastic ep scattering in perturbation theory, Sov. J. Nucl. Phys. 15 (1972), 438, [Yad. Fiz. 15 (1972), 781]

[148] V. V. Sudakov, Vertex parts at very high-energies in quantum electrodynamics, Sov. Phys. JETP 3 (1956), 65, [Zh. Eksp. Teor. Fiz. 30 (1956), 87]

[149] M. H. Seymour, Matrix element corrections to parton shower algorithms, Comput. Phys. Commun. 90 (1995), 95

[150] T. Sjöstrand, S. Mrenna, and P. Z. Skands, PYTHIA 6.4 Physics and Manual, JHEP 05 (2006), 026

[151] S. Höche, S. Schumann, and F. Siegert, Hard photon production and matrix-element parton-shower merging, Phys. Rev. D81 (2010), 034026

[152] S. Plätzer and M. Sjödahl, The Sudakov Veto Algorithm Reloaded, Eur. Phys. J. Plus 127 (2012), 26

[153] L. Lönnblad, Fooling Around with the Sudakov Veto Algorithm, Eur. Phys. J. C73 (2013), 2350

[154] P. A. W. Lewis and G. S. Shedler, Simulation of nonhomogeneous poisson processes by thinning, Naval Research Logistics Quarterly 26 (1979), 403

[155] R. Kleiss and R. Verheyen, Competing Sudakov Veto Algorithms, Eur. Phys. J. C76 (2016), 359

[156] S. Catani, B. R. Webber, and G. Marchesini, QCD coherent branching and semiinclusive processes at large $x$, Nucl. Phys. B349 (1991), 635

[157] S. Alioli et al., NLO vector-boson production matched with shower in POWHEG, JHEP 07 (2008), 060

[158] S. Höche and M. Schönherr, Uncertainties in next-to-leading order plus parton shower matched simulations of inclusive jet and dijet production, Phys. Rev. D86 (2012), 094042

[159] E. Farhi, A QCD Test for Jets, Phys. Rev. Lett. 39 (1977), 1587

[160] A. Heister et al. (ALEPH), Studies of QCD at $e+e$-centre-of-mass energies between 91- $\mathrm{GeV}$ and 209-GeV, Eur. Phys. J. C35 (2004), 457

[161] A. Schälicke and F. Krauss, Implementing the ME+PS merging algorithm, JHEP o7 (2005), 018

[162] F. Krauss et al., Measuring collinear W emissions inside jets, Phys. Rev. D89 (2014), 114006 
[163] J. Alwall et al., The automated computation of tree-level and next-to-leading order differential cross sections, and their matching to parton shower simulations, JHEP 07 (2014), 079

[164] B. Dumont et al., Toward a public analysis database for LHC new physics searches using MADANALYSIS 5, Eur. Phys. J. C75 (2015), 56

[165] V. Bertone, S. Carrazza, and N. P. Hartland, APFELgrid: a high performance tool for parton density determinations, (2016), arXiv: 1605.02070 [hep-ph]

[166] Z. Nagy and Z. Trocsanyi, Multijet cross-sections in deep inelastic scattering at nextto-leading order, Phys. Rev. Lett. 87 (2001), 082001

[167] J. M. Campbell, R. K. Ellis, and W. T. Giele, A Multi-Threaded Version of MCFM, Eur. Phys. J. C75 (2015), 246

[168] V. Khachatryan et al. (CMS), Dijet Azimuthal Decorrelations in pp Collisions at $\sqrt{s}=7 \mathrm{TeV}$, Phys. Rev. Lett. 106 (2011), 122003

[169] V. Khachatryan et al. (CMS), Measurement of dijet azimuthal decorrelation in pp collisions at $\sqrt{s}=8 \mathrm{TeV}$, Submitted to: Eur. Phys. J. C (2016)

[170] E. Gerwick, Recursive prescription for logarithmic jet rate coefficients, Phys. Rev. D88 (2013), 094009

[171] M. Dasgupta et al., Small-radius jets to all orders in QCD, JHEP O4 (2015), 039

[172] S. El Hedri et al., Learning How to Count: A High Multiplicity Search for the LHC, JHEP o8 (2013), 136

[173] M. L. Mangano et al., Physics at a $100 \mathrm{TeV}$ pp collider: Standard Model processes, (2016), arXiv: 1607.01831 [hep-ph]

[174] E. Bothmann et al., Aspects of perturbative QCD at a $100 \mathrm{TeV}$ future hadron collider, Phys. Rev. D94 (2016), 034007

[175] D. d'Enterria and T. Pierog, Global properties of proton-proton collisions at $\sqrt{s}=100$ $\mathrm{TeV}$, JHEP o8 (2016), 170

[176] M. Rubin, G. P. Salam, and S. Sapeta, Giant QCD K-factors beyond NLO, JHEP o9 (2010), 084

[177] S. Chatrchyan et al. (CMS), Event shapes and azimuthal correlations in $Z+$ jets events in pp collisions at $\sqrt{s}=7 \mathrm{TeV}$, Phys. Lett. B722 (2013), 238 


\section{Danksagung}

Zunächst möchte ich meinem Doktorvater Steffen Schumann für seine Betreuung während der vergangenen drei Jahre und auch schon während der Masterarbeit danken. Durch diese Unterstützung konnten beide Arbeiten und das viele Drumherum unter den besten Bedingungen stattfinden. Als sein erster Doktorand hoffe ich, dass noch viele weitere folgen werden und so von ihm als Doktorvater profitieren können. Mein herzlicher Dank gilt auch Arnulf Quadt, dem Zweitbetreuer dieser Arbeit.

Ich danke der SHE RPA-Kollaboration für die gute Zusammenarbeit, insbesondere Marek Schönherr, Frank Krauss, Jennie Thompson und Silvan Kuttimalai. Marek Schönherr zögerte nicht einmal, mir technische Details des nicht immer für sich sprechenden SHERPACodes zu erläutern. Frank Krauss und Marek Schönherr waren außerdem gute Gastgeber bei meinem Besuch in Durham, und letzterer noch ein weiteres Mal in Zürich. Frank Siegert danke ich für seine Testläufe meiner Reweighting-Implementation.

Nathan Hartland möchte ich für die Zusammenarbeit an MCGRID danken, ebenso wie für die arbeits- und freizeitintensive Zeit während meines Aufenthalts in Edinburgh. Zum Glück durfte ich die Stadt noch ein weiteres Mal besuchen, für diese zweite Einladung möchte ich Luigi Del Debbio und den anderen Mitarbeitern des Higgs Centre danken. Auch meinen Gastgebern am CERN, Tancredi Carli und Klaus Rabbertz, gilt mein Dank.

In den letzten Tagen dieser Arbeit haben Jennie und Timon wertvolle Korrekturen vorgenommen. Danke.

Ebenso danke ich allen, die am Institut in Göttingen für die nötige tägliche Ablenkung gesorgt haben, Piero und Matteo, meinen langjährigen Bürokollegen Julia, Knut und Konstantin und vielen anderen. Ich danke außerdem dem Sekretariat für organisatorische Hilfe, insbesondere Bernadette Tyson, die zudem mit Pflanzen und lieben Worten Farbe ins Büro gebracht hat.

Während einer Doktorarbeit bleibt zu wenig Zeit für gute Freunde, und ich danke allen für ihr Verständnis, wenn ich mich zu wenig gemeldet und gezeigt habe. Das gilt auch und vor allem für meine Eltern und meinen Bruder. Ich danke Euch für Eure Unterstützung.

Mein besonderer Dank gilt meiner Freundin, ihrer lebensfrohen Unterstützung, den zahlreichen Gedanken, die sie sich rund um die Arbeit und mich gemacht hat, und den Halt, den sie mir so geben konnte. 